\title{
Measurement of Neutral Current Neutral Pion Production on Carbon in a Few-GeV Neutrino Beam
}

\section{Yoshinori Kurimoto}

Department of Physics, Graduate School of Science Kyoto University

January, 2010

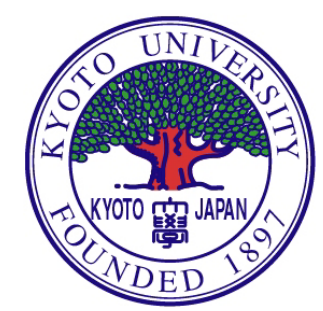





\title{
Measurement of Neutral Current Neutral Pion Production on Carbon in a Few-GeV Neutrino Beam
}

\author{
A dissertation \\ submitted in partial fulfillment of the requirements \\ for the Degree of Doctor of Science \\ in the Graduate School of Science, Kyoto University
}

\section{Yoshinori Kurimoto}

Department of Physics, Graduate School of Science

Kyoto University

January, 2010 


\begin{abstract}
Understanding of the $\pi^{0}$ production via neutrino-nucleus neutral current interaction in the neutrino energy region of a few $\mathrm{GeV}$ is essential for the neutrino oscillation experiments.

In this thesis, we present a study of neutral current $\pi^{0}$ production from muon neutrinos scattering on a polystyrene $\left(\mathrm{C}_{8} \mathrm{H}_{8}\right)$ target in the SciBooNE experiment. All neutrino beam data corresponding to $0.99 \times 10^{20}$ protons on target have been analyzed.

We have measured the cross section ratio of the neutral current $\pi^{0}$ production to the total charge current interaction and the $\pi^{0}$ kinematic distribution such as momentum and direction. We obtain $[7.7 \pm 0.5$ (stat.) \pm 0.5 (sys.) $] \times 10^{-2}$ as the ratio of the neutral current neutral pion production to total charged current cross section; the mean energy of neutrinos producing detected neutral pions is $1.1 \mathrm{GeV}$. The result agrees with the ReinSehgal model, which is generally used for the Monte Carlo simulation by many neutrino oscillation experiments. We achieve less than $10 \%$ uncertainty which is required for the next generation search for $\nu_{\mu} \rightarrow \nu_{e}$ oscillation. The spectrum shape of the $\pi^{0}$ momentum and the distribution of the $\pi^{0}$ emitted angle agree with the prediction, which means that not only the Rein-Sehgal model but also the intra-nuclear interaction models describe our data well.

We also measure the ratio of the neutral current coherent pion production to total charged current cross section to be $(1.17 \pm 0.23) \times 10^{-2}$ based on the Rein and Sehgal model. The result gives the evidence for non-zero coherent pion production via neutral current interaction at the mean neutrino energy of $1.0 \mathrm{GeV}$.
\end{abstract}




\section{Acknowledgements}

My heartfelt appreciation goes to my supervisor Prof. Tsuyoshi Nakaya for giving me an opportunity for this research. His leadership as a co-spokesperson of SciBooNE led the experiment toward a success. His encouragement, criticism, and honest advice were of inestimable value for my research. I could learn a lot of things from his attitude to the physics and experiments. I am deeply thankful to Prof. Masashi Yokoyama. His advice as an on-site leader during the detector construction, operation always led us to the best direction to our success. With him, I was very encouraged and able to learn how we make detectors work. These were precious experience for me. I would like express my thanks to Dr. H.-K. Tanaka. His immense efforts contribute to everything of SciBooNE from the detector construction to physics analysis. I would like to thank Prof. Koichiro Nishikawa, my former adviser, who involved me with a series of the exciting neutrino experiments: $\mathrm{K} 2 \mathrm{~K}$ and $\mathrm{T} 2 \mathrm{~K}$.

I would like to acknowledge all the people in the SciBooNE Collaboration. Especially, I would like to give my special thanks to Dr. Morgan Wascko who welded together a team of collaborators as a co-spokesperson of SciBooNE. He take care of not only SciBooNE but also our lives in Fermilab by making social events (SciBooNE social club). Thanks to these events, I have no hesitation in communicating people in English (apart from my English skill). I am grateful to Prof. Y. Hayato for coming to Fermilab to give me advice on my work about the SciBooNE data acquisition system. I enjoyed learning a lot of new things about DAQ. I am grateful to Dr. R. Tesarek for leading us as a project manager. I am thankful to Dr. L. Ludovici and Dr. R. Napora for leading the construction and commissioning works for the EC and MRD, respectively. I would like to thank Dr. M. Sorel, Dr. G. Zeller for giving me appropriate advice on my analysis. I really thank Dr. K. Hiraide and Y. Nakajima for staying with me in the same house for more than two years as well as working together on SciBooNE. Discussion about the physics analyses and the detector troubles with them were priceless experience for me. I was glad that they did not complain about my noise and lots of my stuff in our house. I am grateful to Dr. T. Katori. I was very shocked by not only his knowledge and enthusiasm about physics but also how much he enjoys his private life. I could learn a lot of things by taking with him. I am very glad that he asks us to go to Chicago several times. I would like to express my thanks to Dr. K. Mahn for helping me in not only SciBooNE but also my whole life in US. Despite of our poor English, she actively talked to us and arranging several social events for us. Thanks to her kindness, we could enjoyed staying in US. I really thank Dr. H. Takei, Dr. G. Mitsuka, J. Walding, Dr. C. Mariani, C. Giganti, J. Alcaraz, J. Catala, A. Hanson, and G. Cheng for working hard and drinking a lot with me. I spent precious days with them during two years' stay at FNAL, and without them the experiment could not have been achieved in such a short time scale. I am thankful 
to K. Matsuoka, H. Kubo, D. Orme, Y. Kobayashi, S. Masuike, and S. Mizugashira for helping us construct and/or de-commission the detector.

I would also like to express my thanks to people outside the collaboration who supported our experiment. I would like to thank J. Kubota, Y. Kurosawa, T. Nobuhara, M. Taguchi, Dr. Y. Takenaga, C. Ishihara, T. Koike, and Y. Maruyama for helping us dismantle fibers from SciBar at KEK. I would like to thank H. Kawamuko, S. Gomi, T. Usuki, N. Kobayashi, K. Sugiya, Y. J. Lee, J. E. Jung, and C. S. Moon for helping us install fibers into SciBar at FNAL. I gratefully thank people in Accelerator Division at FNAL for providing us a proton beam in good condition throughout the data-taking period. I am thankful to the MiniBooNE Collaboration for sharing not only neutrino beam but also experimental shifts. I deeply thank M. Otani and A. Murakami for helping us de-commisson the detector.

I am grateful to S. Kunori and K. Kunori for inviting us to plenty of house parties during our stay at FNAL. Every time, we ate, drank and sing a lot, and spent delightful time.

I wish to extend my thanks to the $\mathrm{K} 2 \mathrm{~K}$ and $\mathrm{T} 2 \mathrm{~K}$ Collaborations, the first experiments which I joined. Especially, I would like to thank Dr. H. Maesaka, Dr. M. Hasegawa, Dr. S. Yamamoto and Dr. Y. Takubo for giving me advice as experts for K2K-SciBar. I gratefully thank Prof. A. K. Ichikawa for sharing the office and pushing me to complete this thesis after I was back in Kyoto from Fermilab. I am also thankful to Prof. T. Kobayashi, Prof. T. Ishii, Dr. T. Ishida, Dr. S. Tada, Dr. T. Nakadaira, Dr. T. Sekiguchi, Dr. I. Kato.

I am thankful to members of High Energy Physics Group in Kyoto University: Prof. N. Sasao, Prof. T. Nomura, Dr. H. Nanjo, M. Suehiro, H. Yokoyama, Dr. Y. Honda, Dr. K. Mizouchi, Dr. T. Sumida, Dr. A. Minamino, Dr. K. Nitta, H. Morii, Dr. N. Taniguchi, T. Shirai, K. Takezawa, K. Ezawa, K. Shiomi, N. Kawasaki, N. Nagai, T. Masuda, K. Ieki, D. Naito, Y. Maeda, T. Kikawa, K. Suzuki, G. Takahashi and S. Takahashi.

I would like to express my thanks to A. Nakao, K. Nakagawa, M. Hiraoka, and the other secretaries of Kyoto University for taking care of every business.

I would like to acknowledge supports from the Japan Society for Promotion of Science (JSPS), the Japan/U.S. Cooperation Program in the field of High Energy Physics, Fermi National Accelerator Laboratory, and the global COE program "The Next Generation of Physics, Spun from Universality and Emergence". This work was supported from the MEXT and JSPS (Japan), the INFN (Italy), the Ministry of Science and Innovation and CSIC (Spain), the STFC (UK), and the DOE and NSF (USA).

Finally, I would like to express my special appreciation to my parents for various supports throughout my life. 


\section{Contents}

$\begin{array}{lr}\text { Acknowledgments } & 2\end{array}$

1 Introduction $\quad 7$

1.1 Neutrinos and Neutrino Oscillations . . . . . . . . . . . . . . . 7

1.1.1 Neutrinos and Their Masses . . . . . . . . . . . . . . . 7

1.1.2 Phenomenology of Neutrino Oscillations . . . . . . . . . . . 7

1.1.3 Summary of Neutrino Oscillation Measurements . . . . . . . . . . 8

1.1.4 Next Step of Neutrino Oscillation Experiment . . . . . . . . . . . . 9

1.2 Neutrino-nucleus Interaction . . . . . . . . . . . . . . . . . 10

1.3 Neutral Current $\pi^{0}$ Production . . . . . . . . . . . . . . . . . . . 10

1.3.1 Importance of $\mathrm{NC}^{0}$ Production . . . . . . . . . . . . . . . . . 11

1.3.2 $\pi^{0}$ Production Mechanisms and Measurements . . . . . . . . . . . . 13

1.4 Overview of This Thesis . . . . . . . . . . . . . . . . . . . 18

2 SciBooNE Experiment 19

2.1 Overview of SciBooNE . . . . . . . . . . . . . . . . . . . . . . . . . 19

2.2 Physics Motivations of SciBooNE . . . . . . . . . . . . . . 20

2.2.1 Precise Measurements of Neutrino-nucleus Cross Sections . . . . . . 20

2.2.2 Measurements of Antineutrino-nucleus Cross Sections . . . . . . . . 20

2.2.3 BNB Neutrino Flux Measurements . . . . . . . . . . . . . . . . . 21

3 Experimental Setup and Data Set $\quad 22$

3.1 Booster Neutrino Beam . . . . . . . . . . . . . . . . 22

3.1.1 Target and Magnetic Focusing Horn . . . . . . . . . . . . . . . . . 22

3.1.2 Decay Region and Absorber . . . . . . . . . . . . . . 23

3.2 SciBooNE Detector . . . . . . . . . . . . . . . . . . . . . 23

3.2.1 Scintillator Bar Tracker (SciBar) . . . . . . . . . . . . . . 24

3.2.2 Electromagnetic Calorimeter (EC) . . . . . . . . . . . . . 28

3.2 .3 Muon Range Detector (MRD) . . . . . . . . . . . . . . . . . . 29

3.2.4 Trigger and Data Acquisition (DAQ) System . . . . . . . . . . . . . 30

3.2.5 Detector Coordinate . . . . . . . . . . . . . . . 34

3.3 Data Set . . . . . . . . . . . . . . . . . . 34

3.3.1 Data Quality Cuts . . . . . . . . . . . . . 35

3.3.2 Summary of Data-taking . . . . . . . . . . . . . . 36 
4 Monte Carlo Simulation $\quad 37$

4.1 Neutrino Beam Simulation . . . . . . . . . . . . . . . . . 37

4.1.1 Simulation of Meson Productions . . . . . . . . . . . . 37

4.1.2 Simulation of Meson Decays . . . . . . . . . . . . . . . . 38

4.1.3 Neutrino Beam Flux Prediction at SciBooNE . . . . . . . . . . . 38

4.1.4 Systematic Uncertainties in the Neutrino Flux Prediction . . . . . . 38

4.2 Neutrino Interaction Simulation (NEUT) . . . . . . . . . . . . . . . . 39

4.2 .1 Neutrino-Nucleus Interactions . . . . . . . . . . . . . . . . 39

4.2 .2 Intra-nuclear Interactions . . . . . . . . . . . . . . . . . . . 47

4.3 Detector Simulation . . . . . . . . . . . . . . . . . . 47

4.3.1 Simulation of Detector Responses . . . . . . . . . . . . . . . 47

4.3.2 Simulation of Pion Interaction in Detector . . . . . . . . . . . . . 48

4.3.3 Neutrino Interactions in the Surrounding Material . . . . . . . . . . 48

5 Event Selection for Neutral Current $\pi^{0}$ Production 49

5.1 Overview . . . . . . . . . . . . . . . . . . . . . . 49

5.2 Signal Definition . . . . . . . . . . . . . . . . . . . . . 49

5.3 Particle Reconstruction . . . . . . . . . . . . . . . . . . . . . 51

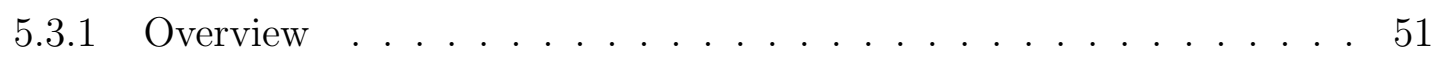

5.3.2 SciBar Track Reconstruction . . . . . . . . . . . . . . . . 51

5.3 .3 Particle Identification Parameter . . . . . . . . . . . . . . 53

5.3 .4 Extended Track . . . . . . . . . . . . . . . . . . . . . 53

5.3 .5 SciBar-EC Matching . . . . . . . . . . . . . . . . . 54

5.3 .6 SciBar-MRD Matching . . . . . . . . . . . . . . . . . . 54

5.4 MC Normalization (Charged-Current Event Sample) . . . . . . . . . . . 54

5.5 Event Selection for $\mathrm{NC} \pi^{0} \ldots \ldots \ldots \ldots \ldots \ldots$

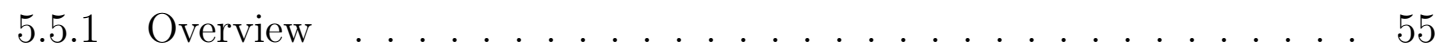

5.5 .2 Number of Tracks . . . . . . . . . . . . . . . . . . . . . 56

5.5 .3 Fiducial Volume Cut . . . . . . . . . . . . . . . . . 56

5.5 .4 First Layer Veto . . . . . . . . . . . . . . . . . . . . 57

5.5.5 Side Escaping Track Rejection Cut . . . . . . . . . . . . . . . . 57

5.5 .6 Decay Electron Rejection Cut . . . . . . . . . . . . . . . . 59

5.5.7 Track Disconnection Cut . . . . . . . . . . . . . . . 60

5.5 .8 Electron Catcher Cut . . . . . . . . . . . . . . . . . 60

5.5.9 The Number of Extended Tracks . . . . . . . . . . . . . . . 62

$5.5 .10 \pi^{0}$ Vertex Cut . . . . . . . . . . . . . . . . . 64

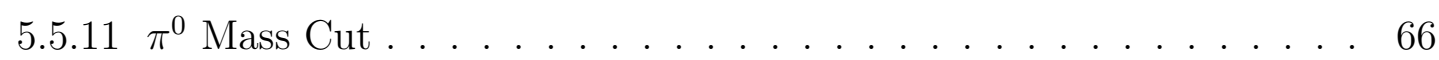

5.5 .12 Event Summary . . . . . . . . . . . . . . . . 66

6 Study of Neutral Current $\pi^{0}$ Production $\quad 68$

6.1 Overview . . . . . . . . . . . . . . . . . . 68

6.2 Neutral Current $\pi^{0}$ Cross Section . . . . . . . . . . . . . . . . 68

6.2.1 Neutral Current $\pi^{0}$ Production ................... 68

6.2.2 Total Charged Current Interaction . . . . . . . . . . . . . . 69

6.2.3 Cross Section Ratio . . . . . . . . . . . . . . . . . . . . . . . . . 70

6.2 .4 Systematic Uncertainty . . . . . . . . . . . . . . . . . . . 70

6.3 Reconstructed $\pi^{0}$ Kinematics . . . . . . . . . . . . . . . . . . 74 
6.3.1 Reconstructed $\pi^{0}$ Mass . . . . . . . . . . . . . . . . . . . 74

6.3.2 Reconstructed $\pi^{0}$ Momentum . . . . . . . . . . . . . . . . . . 75

6.3.3 Reconstructed $\pi^{0}$ Angle . . . . . . . . . . . . . . . . . . . 77

6.3.4 Reconstructed Gamma Angle . . . . . . . . . . . . . . . . . 77

6.3.5 Extraction of True $\pi^{0}$ Momentum and Angular Distribution . . . . 78

6.4 Coherent Pion Production . . . . . . . . . . . . . . . . 81

6.4.1 Vertex Activity . . . . . . . . . . . . . . . . 81

6.4.2 Coherent Pion Extraction . . . . . . . . . . . . . . 83

6.5 Summary . . . . . . . . . . . . . . . . . . . 88

6.6 Discussion . . . . . . . . . . . . . . . . . . . . . . . . . . . . . 88

6.6.1 Impact of our Results on Neutrino Physics and Future Prospects . . 88

6.6.2 Increasing the Detection Efficiency . . . . . . . . . . . . . . . 89

\section{Conclusions}

A The Gamma and $\pi^{0}$ Reconstruction $\quad 95$

A.1 Extended Track . . . . . . . . . . . . . . . . . . . . . . . 95

A.1.1 Track Merge . . . . . . . . . . . . . . . . . . 95

A.1.2 Energy Reconstruction . . . . . . . . . . . . . . . . . . 95

A.1.3 Direction Reconstruction . . . . . . . . . . . . . . . . 98

A.2 SciBar-EC Matching . . . . . . . . . . . . . . . . . . . 99

A.3 Performance of Gamma Reconstruction . . . . . . . . . . . . . . . . . . . 99

A.3.1 Gamma Angular Resolution . . . . . . . . . . . . . . . . . . . . . . 100

A.3.2 Gamma Energy Resolution . . . . . . . . . . . . . . . . . . . . . 100

A.4 Performance of $\pi^{0}$ Reconstruction . . . . . . . . . . . . . . . . . 104

A.4.1 $\pi^{0}$ Vertex Resolution . . . . . . . . . . . . . . . . . . . . . . . 104

A.4.2 $\pi^{0}$ Angle Resolution . . . . . . . . . . . . . . . . . . . . . 104

A.4.3 $\pi^{0}$ Mass Resolution and Momentum Resolution . . . . . . . . . . . 104

B Neutrino Interaction In The Surrounding Material 108

B.1 Dirt material . . . . . . . . . . . . . . . . . . . . . 108

B.2 The area of neutrino events . . . . . . . . . . . . . . 109

C MC Expectation with Systematic Uncertainty 110

D Charged Current Coherent Pion Production 112

D.1 Event Selection of CC Coherent Pion Production . . . . . . . . . . . . 112

D.2 Study of CC Coherent Pion Production for Neutrino and Antineutrino . . 113

D.2.1 $\nu_{\mu}$ CC Coherent Pion Production . . . . . . . . . . . . . . . 113

D.2.2 $\bar{\nu}_{\mu}$ CC Coherent Pion Production . . . . . . . . . . . . 115

D.3 Summary . . . . . . . . . . . . . . . . . . . . . . 117 


\section{Chapter 1}

\section{Introduction}

\subsection{Neutrinos and Neutrino Oscillations}

\subsubsection{Neutrinos and Their Masses}

Neutrino was postulated by Pauli in 1930 in order to explain the continuum electron energy spectrum from the $\beta$ decay and its observation was achieved by Reines and Cowan in 1958 for the first time.

In the standard model of particle physics, neutrino masses had been set to zero. However, in 1998, the Super-Kamiokande observed neutrino oscillation, which indicated finite neutrino masses.

\subsubsection{Phenomenology of Neutrino Oscillations}

In general, a flavor eigenstate of neutrino, $\mid \nu_{\alpha}>(\alpha=e, \mu, \tau)$, can be expressed as a superposition of mass eigenstates, $\mid \nu_{i}>(i=1,2,3)$;

$$
\left|\nu_{\alpha}>=\sum_{i} U_{\alpha, i}\right| \nu_{i}>
$$

where $U_{\alpha i}$ is an element of a $3 \times 3$ unitary matrix $U$ which is referred to as MakiNakagawa-Sakata (MNS) matrix [1]. The matrix is expressed using four independent parameters:three mixing angles, $\theta_{12}, \theta_{23}, \theta_{13}$, and one complex phase $\delta$;

$$
\begin{aligned}
U & =\left(\begin{array}{ccc}
1 & 0 & 0 \\
0 & c_{23} & s_{23} \\
0 & -s_{23} & c_{23}
\end{array}\right)\left(\begin{array}{ccc}
c_{13} & 0 & s_{13} e^{-i \delta} \\
0 & 1 & 0 \\
-s_{13} e^{i \delta} & 0 & c_{13}
\end{array}\right)\left(\begin{array}{ccc}
c_{12} & s_{12} & 0 \\
-s_{12} & c_{12} & 0 \\
0 & 0 & 1
\end{array}\right) \\
& =\left(\begin{array}{ccc}
c_{12} s_{13} & s_{12} c_{13} & s_{13} e^{-i \delta} \\
-s_{12} c_{23}-c_{12} s_{13} s_{23} e^{i \delta} & c_{12} c_{23}-s_{12} s_{13} s_{23} e^{i \delta} & c_{13} s_{23} \\
s_{12} s_{23}-c_{12} s_{13} c_{23} e^{i \delta} & c_{12} s_{23}-s_{12} s_{13} c_{23} e^{i \delta} & c_{13} c_{23}
\end{array}\right),
\end{aligned}
$$

where $c_{i j}=\cos \theta_{i j}$ and $s_{i j}=\sin \theta_{i j}$. In case of $\delta \neq 0$, the MNS matrix includes the imaginary parts, which means the $\mathrm{CP}$ violation in the lepton sector. Hence, the $\delta$ is called the CP phase.

Generated as $\nu_{\alpha}$, the state of neutrino at time $t$ after traveling distance $L$ is expressed as

$$
\left|\nu(t)>=\sum_{i} U_{\alpha i} e^{-t\left(E_{i} t-p_{i} L\right)}\right| \nu_{i}>
$$


where $E_{i}$ and $p_{i}$ are the energy and momentum of $\nu_{i}$ in the laboratory frame, respectively. In practice, neutrino is extremely relativistic due to the tinniness of the mass, and thus we can make following approximations:

$$
\begin{array}{r}
t \sim L, \\
E_{i}=\sqrt{p_{i}^{2}+m_{i}^{2}} \sim p_{i}+\frac{m_{i}^{2}}{2 p_{i}}
\end{array}
$$

Since $\nu_{\alpha}$ is produced with a definite momentum $p$ all of $\nu_{\alpha}$ 's mass eigenstates have a common momentum. Thus, the probability $P\left(\nu_{\alpha} \rightarrow \nu_{\beta}\right)$ that $\nu_{\beta}$ is observed after $\nu_{\alpha}$ travels the distance the distance $L$ is given by

$$
\begin{aligned}
P\left(\nu_{\alpha} \rightarrow \nu_{\beta}\right)= & \left|<\nu_{\beta}\right| \nu(t)>\left.\right|^{2}=\left|\sum_{i} U_{\alpha i} U_{\beta i}^{*} e^{-i p L} e^{-i \frac{m_{i}^{2} L}{2 p}}\right|^{2} \\
= & \delta_{\alpha \beta}-4 \sum_{i>j} \operatorname{Re}\left(U_{\alpha i} U_{\beta i}^{*} U_{\alpha j}^{*} U_{\beta j}\right) \sin ^{2}\left[1.27 \Delta m_{i j}^{2} \frac{L}{E}\right] \\
& -4 \sum_{i>j} \operatorname{Im}\left(U_{\alpha i} U_{\beta i}^{*} U_{\alpha j}^{*} U_{\beta j}\right) \sin ^{2}\left[2.54 \Delta m_{i j}^{2} \frac{L}{E}\right]
\end{aligned}
$$

where $\Delta m_{i j}^{2} \equiv m_{i}^{2}-m_{j}^{2}$ is the mass squared difference between $\nu_{i}$ and $\nu_{j}$ in $\mathrm{eV}^{2}, L$ is in $\mathrm{km}$, and $E$ is in $\mathrm{GeV}$. The sign of the last term in Eq. 1.7 is + instead of - in the case of the expression for antineutrino. Because of the condition $\Delta m_{12}^{2}+\Delta m_{23}^{2}+\Delta m_{31}^{2}=0$ to be imposed, the number of independent parameters for neutrino oscillations is six in the case of three lepton generations: three mixing angles, $\left(\theta_{12}, \theta_{23}, \theta_{31}\right)$, one $\mathrm{CP}$ phase, $\delta$, and any two out of three mass squared difference, $\Delta m^{2}$ 's.

\subsubsection{Summary of Neutrino Oscillation Measurements}

There are many neutrino oscillation measurements such as atmospheric neutrino observations, solar neutrino observations, reactor neutrino experiments and accelerator neutrino experiments. Figure 1.1 shows allowed or excluded regions from various experiments. In summary, there are two allowed regions:

1. Atmospheric region: $\Delta m_{23}^{2} \sim 2.5 \times 10^{-3} \mathrm{eV}^{2}, \theta_{23} \sim 45$ degrees

The neutrino oscillation in the atmospheric region was discovered with the $\nu_{\mu} \rightarrow \nu_{x}$ oscillation by the Super-Kamiokande (SK) group [2]. This is the first discovery of the neutrino oscillation. The result have been confirmed by two long base-line accelerator neutrino experiments (K2K [3] and MINOS [4]).

2. Solar region: $\Delta m_{12}^{2} \sim 8 \times 10^{-5} \mathrm{eV}^{2}, \theta_{12} \sim 30$ degrees

The neutrino oscillation in the solar region was discovered with $\nu_{e} \rightarrow \nu_{x}$ oscillation by solar neutrino experiments ( [5],SNO [6] and SK [7]) and confirmed by a reactor neutrino experiment;KamLAND [8].

Meanwhile, the $\theta_{13}$ and $\delta$ have never been measured to be nonzero. Some reactor experiments $[9,10]$ searches $\theta_{13}$ with the $\nu_{e} \rightarrow \nu_{x}$ oscillation and gives the $90 \%$ C.L. (Confidence Level) upper limit as

$$
\sin ^{2} 2 \theta_{13}<0.15
$$




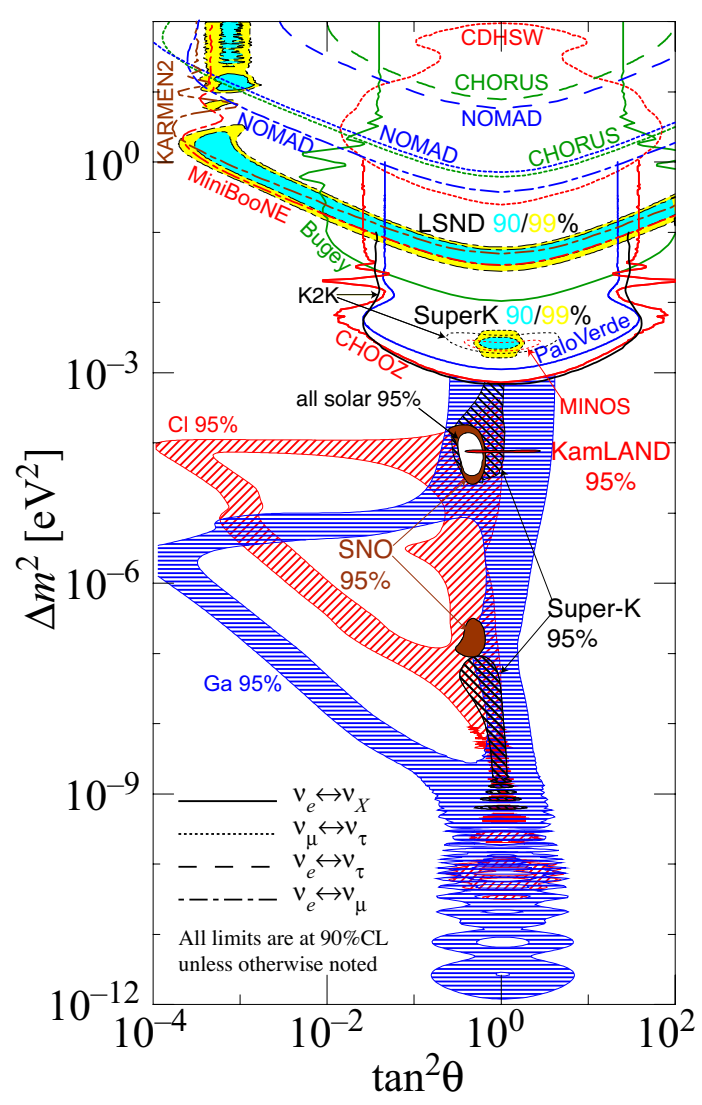

http://hitoshi.berkeley.edu/neutrino

Figure 1.1: Allowed or excluded regions in the $\tan ^{2} \theta-\Delta m^{2}$ plane from various experiment; There are two allowed regions around $\Delta m^{2} \sim 10^{-3}\left(\tan ^{2} \theta \sim 1\right)$ and $\Delta m^{2} \sim 10^{-5}$ $\left(\tan ^{2} \theta \sim 1 / 3\right)$ which are corresponding to the atmospheric and solar regions, respectively. In addition, there is one allowed region around $\Delta m^{2} \sim 1$. However, most of the region is excluded by other experiments such as MiniBooNE.

when $\Delta m_{23}^{2} \sim 2.4 \times 10^{-3} \mathrm{eV}^{2}[11]$. The $\theta_{13}$ can be also constrained by searching the $\nu_{\mu} \rightarrow \nu_{e}$ oscillation in long base-line accelerator neutrinos. This channel has been studied by K2K [12] and MINOS [13]. The signal of $\nu_{\mu} \rightarrow \nu_{e}$ oscillation has not been observed so far.

\subsubsection{Next Step of Neutrino Oscillation Experiment}

As shown above, $\theta_{12}$ and $\theta_{23}$ are measured to be nonzero value and confirmed by several experiments with different methods. However, $\theta_{13}$ has never been measured to be nonzero value and just upper limit is known so far. As shown in Eq. 1.2, the CP phase $\delta$ enters only the MNS matrix in combination with $\sin \theta_{13}$. Hence, $\delta$ never appear on the expressions of the transition probabilities for any possible neutrino oscillations if $\theta_{13}$ is zero. In case of non-zero $\theta_{13}$, the CP phase can be extracted by calculating the asymmetry of the transition probability for the $\nu_{\mu} \rightarrow \nu_{e}$ and $\bar{\nu}_{\mu} \rightarrow \bar{\nu}_{e}$ oscillations as

$$
A=\frac{P\left(\nu_{\mu} \rightarrow \nu_{e}\right)-P\left(\bar{\nu}_{\mu} \rightarrow \bar{\nu}_{e}\right)}{P\left(\nu_{\mu} \rightarrow \nu_{e}\right)+P\left(\bar{\nu}_{\mu} \rightarrow \bar{\nu}_{e}\right)}=\frac{\Delta m_{12}^{2} L}{4 E_{\nu}} \cdot \frac{\sin 2 \theta_{12}}{\sin \theta_{13}} \cdot \sin \delta .
$$


Therefore, measuring $\theta_{13}$ is one of the main motivations for the neutrino oscillation experiments.

Since the probability of the $\nu_{\mu} \rightarrow \nu_{e}$ transition is small, the most plausible scenario of the $\nu_{\mu} \rightarrow \nu_{x}$ oscillation is that muon neutrinos oscillate to tau neutrinos $\left(\nu_{\mu} \rightarrow \nu_{\tau}\right)$. However, the $\nu_{\mu} \rightarrow \nu_{\tau}$ transition is not observed yet. The detection of $\nu_{\tau} \mathrm{s}$ is difficult because their energy threshold for charged current $\tau$ production $(3.4 \mathrm{GeV})$ is higher than the neutrino energy maximizing the $\nu_{\mu} \rightarrow \nu_{x}$ oscillation in K2K, T2K and MINOS. The identification of $\nu_{\tau}$ is also difficult because $\tau$ s decay to multiple particles in many ways within short life time $(\sim 0.3 \mathrm{ps})$. No observation of the $\nu_{\mu} \rightarrow \nu_{\tau}$ transition makes it possible alternative scenario of the $\nu_{\mu} \rightarrow \nu_{x}$ oscillation that muon neutrinos oscillate with sterile neutrinos $\left(\nu_{s}\right)$. The sterile neutrinos do not have neither charged current nor neutral current interactions. Since the number of the light neutrino flavors interacting via weak current is measured to be three with the decay width of $Z^{0}$, any additional light neutrino must be sterile neutrino. The search for sterile neutrino is also the motivations for the neutrino oscillation experiments.

\subsection{Neutrino-nucleus Interaction}

The long baseline (LBL) neutrino oscillation experiments play important roles in further understandings of the neutrino oscillation. To measure $\theta_{13}$ by searching $\nu_{\mu} \rightarrow \nu_{e}$ oscillation, the $\mathrm{T} 2 \mathrm{~K}$ experiment recently started [14] and the $\mathrm{NO} \nu \mathrm{A}$ [15] experiment is now under construction aiming at the first run in 2014. The MINOS experiment [11] is currently running mainly for the precise measurements of $\nu_{\mu} \rightarrow \nu_{x}$ and $\bar{\nu}_{\mu} \rightarrow \bar{\nu}_{x}$ oscillations. In addition, such LBL experiments can perform sterile neutrino search.

For the best performance of the LBL experiments, understandings of neutrino-nucleus interaction in the few-GeV neutrino energy range are important. In such neutrino energy range, a neutrino interacts with nucleons in the nucleus (or entire nucleus) in the following processes via charged current $(\mathrm{CC})$ and neutral current $(\mathrm{NC})$ :

- quasi-elastic scattering $\left(\nu N \rightarrow \ell N^{\prime}\right)$

- single pion production $\left(\nu N \rightarrow \ell N^{\prime} \pi\right.$, or $\left.\nu A \rightarrow \ell A \pi\right)$

- deep inelastic scattering $\left(\nu N \rightarrow \ell N^{\prime}+\right.$ hadrons $)$

where $N$ and $N^{\prime}$ are the nucleons (proton or neutron), $A$ is the nucleus, $\ell$ is the lepton.

In general, there are only a handful of cross section measurements in the few-GeV neutrino energy range, and their precision is limited by small statistics. Therefore, the precise measurements of neutrino-nucleus interactions are desired. Among the several interaction processes, the $\pi^{0}$ production via neutral current $\left(\mathrm{NC} \pi^{0}, \nu_{\mu}+N \rightarrow \nu_{\mu}+\pi^{0}+N^{\prime}\right)$ is the one of the most important processes for the $\nu_{\mu} \rightarrow \nu_{e}$ oscillation search.

\subsection{Neutral Current $\pi^{0}$ Production}

In this section, we describe the importance of $\mathrm{NC} \pi^{0}$, the $\pi^{0}$ production mechanisms and the measurement of $\mathrm{NC} \pi^{0}$. 


\subsubsection{Importance of $\mathrm{NC} \pi^{0}$ Production}

The $\mathrm{NC} \pi^{0}$ production is the key of the next step of the neutrino oscillation experiments described in Section 1.1.4. We describe the importance of $\mathrm{NC}^{0}$ production in detail below.

\section{Background for the $\nu_{\mu} \rightarrow \nu_{e}$ Search}

Here, we focus on the search for $\nu_{\mu} \rightarrow \nu_{e}$ oscillation in the T2K (Tokai-to-Kamioka) experiment. The T2K experiment is a new long-baseline neutrino experiment using the Super-Kamiokande (SK) as the far detector. In T2K, the charged current quasi-elastic (CCQE, $\nu_{e}+n \rightarrow e+p$ ) interaction is used to identify the $\nu_{e}$ signal at SK. Since SK is a ring-imaging water Cherenkov detector, the electron from $\nu_{e}$ is detected as a electronlike (e-like) cherenkov ring. Typically the momentum of a recoil proton in the CCQE interaction is below the Cherenkov threshold in the water. Therefore only the electron, visible as a single-ring e-like event, is the signature of $\nu_{e}$ appearance. The probability of

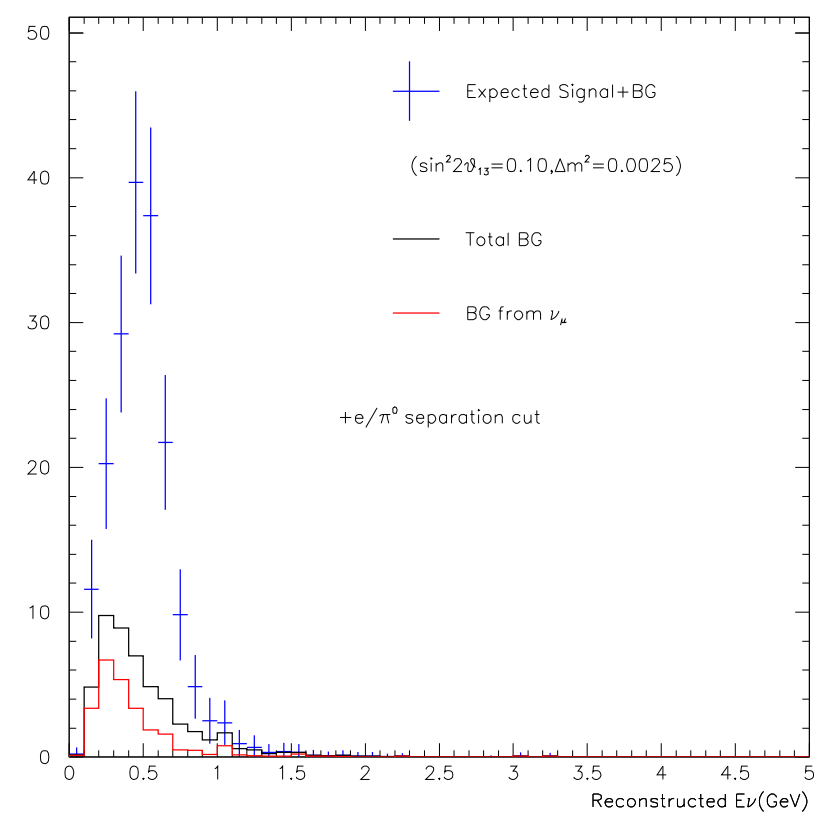

Figure 1.2: The $\nu_{e}$ spectrum at SK with $\left(\sin ^{2} 2 \theta_{13}, \Delta m^{2}\right)=\left(0.1,2.5 \times 10^{-3} \mathrm{eV}^{2}\right)$. The sum of $\nu_{e}$ signals and backgrounds is shown by the dots with error bars, and two histograms show the total background and the $\nu_{\mu}$ background, respectively.

the $\nu_{\mu} \rightarrow \nu_{e}$ oscillation is proportional to $\sin ^{2} 2 \theta_{13}$, which is small (Eq. 1.8). Therefore, precise understanding of the background is crucial. Figure 1.2 shows the expected $\nu_{e}$ energy spectrum at SK with $\left(\sin ^{2} 2 \theta_{13}, \Delta m^{2}\right)=\left(0.1,2.5 \times 10^{-3} \mathrm{eV}^{2}\right)$ with the background contamination. In the figure, the sum of $\nu_{e}$ signals and backgrounds is shown by the dots with error bars, and two histograms show the total background and the $\nu_{\mu}$ background, respectively. As shown in the figure, the $\nu_{\mu}$ background is the one of main backgrounds. The main contribution of the $\nu_{\mu}$ background is the neutral current $\pi^{0}\left(\mathrm{NC} \pi^{0}\right)$ production by $\nu_{\mu}$. In SK, a single $\pi^{0}$ decaying into two gamma rays can be classified as a singlering, e-like event ( $\nu_{e}$ candidate), when one gamma ray is not reconstructed due to highly 
asymmetric energies or a small opening angle between the two gamma rays. Non $\nu_{\mu}$ background is coming from the intrinsic $\nu_{e} \mathrm{~s}$, which are produced in the neutrino beam production not by the $\nu_{\mu} \rightarrow \nu_{e}$ oscillation.

Figure 1.3 shows the T2K's sensitivity to $\sin ^{2} 2 \theta_{13}$ as a function of the beam exposure whose unit is $22.5 \mathrm{kt}\left(\mathrm{SK}^{\prime} \mathrm{s}\right.$ fiducial volume) $\times$ one year. The intensity of the primary proton beam is assumed to be $750 \mathrm{~kW}$. The three lines show three different levels of uncertainties in the subtraction of the $\mathrm{NC} \pi^{0}$ and beam intrinsic $\nu_{e}$. For these exposures, the difference between $10 \%$ and $0 \%$ uncertainties is minor, but between $10 \%$ and $20 \%$ there is a noticeable change. For this reason a $10 \%$ uncertainty on the $\mathrm{NC} \pi^{0}$ cross section is desired. As described in Section 1.3.2, there are no measurements of $\mathrm{NC} \pi^{0}$ cross section with less than $10 \%$ uncertainty at the neutrino energy of T2K $(\sim 0.8 \mathrm{GeV}) *$

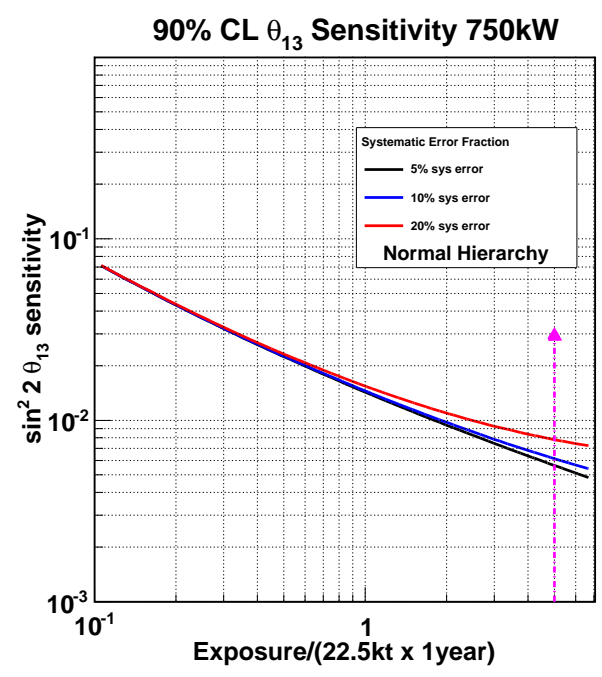

Figure 1.3: The expected $90 \%$ Confidence Level (CL) sensitivities for measuring $\sin ^{2} 2 \theta_{13}$ for uncertainties $0 \%$ (bottom curve), $10 \%$ (middle curve) and $20 \%$ (top curve) in background subtraction.

\section{Signal for the Sterile Neutrino Search}

A single $\pi^{0}$ event is a good signature of $\mathrm{NC}$ interactions in the $\mathrm{GeV}$ region especially for a water cherenkov detector such as SK because a $\pi^{0}$ decay is clearly identified as two e-like Cherenkov rings. The single $\pi^{0}$ production rate by the T2K neutrino beam or atmospheric neutrinos could be usable to distinguish between the $\nu_{\mu} \rightarrow \nu_{\tau}$ and $\nu_{\mu} \rightarrow \nu_{s}$ oscillation hypotheses. Since the sterile neutrinos $\left(\nu_{s}\right)$ have neither CC nor NC interaction, the NC rate is attenuated in the case of transitions of $\nu_{\mu}$ 's into sterile neutrinos. Meanwhile, the $\mathrm{NC}$ rate does not change in the $\nu_{\mu} \rightarrow \nu_{\tau}$ scenario, and the NC measurements can distinguish the two hypotheses.

*After our result of the $\mathrm{NC} \pi^{0}$ cross section measurement was submitted to Physical Review D (PRD), the MiniBooNE collaboration also submitted the result of the $\mathrm{NC} \pi^{0}$ cross section measurement to PRD [16]. Our measurement and MiniBooNE use the common neutrino beam whose energy is $\sim 0.7 \mathrm{GeV}$ and the uncertainties of their measurement are less than $10 \%$. Hence, there exist two $\mathrm{NC} \pi^{0}$ measurements within a $10 \%$ uncertainty at the similar neutrino energy to T2K. 


\subsection{2 $\pi^{0}$ Production Mechanisms and Measurements}

In the neutrino energy rage of a few $\mathrm{GeV}$, pion production through a neutrino scattering on nuclei principally occurs by two mechanisms; the resonant pion production and the coherent pion production.

\section{Resonant Pion Production}

The larger contribution of the $\pi^{0}$ production comes from incoherent processes in which the neutrino interacts with one of the nucleons in the nucleus. In the energy range of a few $\mathrm{GeV}$, the incoherent process mainly consists of the excitation and subsequent pionic decay of baryonic resonances such as $\Delta$ (1232). Both CC and NC resonant pion productions are possible. Considering $\pi^{0}$ production via neutral current interaction by neutrinos, there are two channels:

$$
\begin{aligned}
& \nu_{\mu} p \rightarrow \nu_{\mu} p \pi^{0} \\
& \nu_{\mu} n \rightarrow \nu_{\mu} n \pi^{0} .
\end{aligned}
$$

There exist several theoretical models. Fogli and Narduli [17] expressed the nucleonresonance transition amplitude with the vector form factors and axial form factors assuming usual hypotheses such as CVC (conserved vector current) and PCAC (partially conserved axial vector current). Then, Fogli and Narduli calculated the cross section of the single pion production considering the resonances with $m_{N \pi}<1.6 \mathrm{GeV}$ and non-resonant component. The Rein and Sehgal [18] summed all resonances up to $m_{N \pi}<2.0 \mathrm{GeV}$, using quark model predictions. Since the Rein and Sehgal model is used in our analyses as well as widely used in neutrino oscillation experiments, we will describe the details in Section 4.2.1. Table 1.1 shows the comparison of the cross section of NC resonant $\pi^{0}$ production between the Rein and Sehgal model and the Fogli and Narduli model. The 10-20 $\%$ difference between them is seen. We note that these models predict the single pion cross section only via the neutrino-nucleon interaction. However, the neutrino targets used for recent experiments are generally not hydrogen but heavier nucleus such as carbon, water, iron and so on. In these cases, the resonance production and its decay could be different from a simple picture of neutrino-nucleon interactions due to nuclear effects such as Fermi motion, Pauli blocking and nuclear potential. Therefore, we need to take the nuclear effects into account. For our analyses, such nuclear effects are implemented with Rein-Sehgal model as described in Chapter 4.

Table 1.1: Comparison of the flux averaged calculated cross-section for the beams of ANL(0.4-6.0 GeV, [19,20]) and Gargamelle(1-10 GeV, [21]) with the Rein and Sehgal (RS) model and the Fogli and Narduli (FN) model (in the unit of $10^{-38} \mathrm{~cm}^{2}$ ).

\begin{tabular}{c|cc|cc}
\hline \hline mode & \multicolumn{2}{|c|}{ ANL(0.4-6.0 GeV) } & \multicolumn{2}{c}{ Gargamelle(1-10 GeV) } \\
\hline & $\mathrm{RS}$ & $\mathrm{FN}$ & $\mathrm{RS}$ & $\mathrm{FN}$ \\
\hline$\sigma\left(\nu p \pi^{0}\right)$ & 0.035 & 0.036 & 0.083 & 0.090 \\
$\sigma\left(\nu n \pi^{0}\right)$ & 0.033 & 0.038 & 0.086 & 0.105 \\
\hline \hline
\end{tabular}


There exist several other model calculation for the single resonant pion production including nuclear effects by Athar [22], Praet [23] and Martini [24]. In Athar's model, the $\Delta$ production cross section via simple neutrino-nucleon interaction is modified by using local density approximation where neutrino interacts with a nucleon moving inside the nucleus of density $\rho(r)$ with its corresponding momentum $\vec{p}$ constrained to be below its Fermi momentum $p_{F}(r)=\left[3 \pi^{2} \rho(r)\right]^{\frac{1}{3}}$. In Praet's model, they include nuclear effect adopting impulse approximation: the nuclear many-body current is replaced by a sum of one-body current operators, and assuming an independent-particle model where the initial-nucleon free Dirac spinor is replaced by a bound-state spinor. Both in Athar's and Praet's model, the width and mass of the $\Delta$ is modified so that the $\Delta$ properties in nuclear medium are included. In the Martini's model, they introduce the nuclear response functions and related them to the hadronic tensor $W^{\mu \nu}$ which is contracted with the lepton tensor as $W^{\mu \nu} L_{\mu \nu}$ to obtain the amplitude. In these three models, only $\Delta$ resonance is taken into account so that the cross section may be underestimated. Figure 1.4 shows the comparison of several theoretical predictions for the charged current single $\pi^{+}$production without final state interaction (FSI, which we will describe later). There are significant discrepancies among the predictions The experimental input on the $\mathrm{NC} \pi^{0}$ cross section is needed to choose or correct theoretical models.

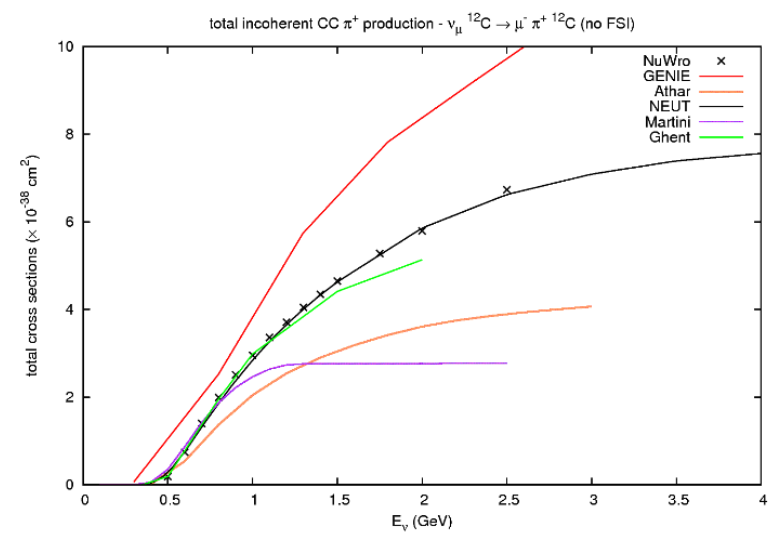

Figure 1.4: The comparison of several theoretical predictions of the total incoherent CC $\pi^{+}$production on Carbon without the final state interaction. Genie [25], Neut (Chapter 4.2) and NuWro [26] are the Monte Carlo generators with the Rein and Sehgal model. (The plots is from the presentation by J.Sobczyk for "Sixth International Workshop on Neutrino-Nucleus Interactions in the Few-GeV Region")

There is another nuclear effect called final state interaction where nucleons and mesons produced in the primary neutrino-nucleon interaction interact with nuclear matter before exiting the nucleus. The final state interactions of nucleons and mesons in the target nucleus could largely modify the number, momenta, directions and charge states of produced particles. Though there exist several theoretical approaches for modeling these processes, their uncertainties are large. Therefore, the kinematics of $\pi^{0}$ s after exiting nucleus are important to compare the theoretical predictions with the experimental observation Therefore, measurements of not only the overall cross section but also emitted $\pi^{0}$ kinematics of $\mathrm{NC} \pi^{0}$ production are important. 
Table 1.2: Past measurements of neutral current resonant $\pi^{0}$ production by neutrinos.

\begin{tabular}{lccc}
\hline \hline Experiment & $E_{\nu}(\mathrm{GeV})$ & Target & Result \\
Gargamelle 1978 & $1-10$ & Propane & $\frac{\sigma\left(\nu_{\mu} p \pi^{0}\right)+\sigma\left(\nu_{\mu} n \pi^{0}\right)}{2 \sigma\left(\mu^{-} p \pi^{0}\right)}=0.45 \pm 0.08$ \\
BNL 1976 & $0.5-14.5$ & Al, C & $\frac{\sigma\left(\nu_{\mu} N \pi^{0}\right)}{\sigma\left(\mu^{-} N^{\prime} \pi^{0}\right)}=0.17 \pm 0.04, \frac{\sigma\left(\nu_{\mu} N \pi^{0}\right)}{\sigma\left(\mu^{+} N^{\prime} \pi^{0}\right)}=0.39 \pm 0.18$ \\
ANL 1981 & $0.4-6.0$ & $\mathrm{H}_{2}, \mathrm{D}_{2}$ & $\frac{\sigma\left(\nu_{\mu} p \pi^{0}\right)}{\sigma\left(\mu^{-} p \pi^{+}\right)}=0.09 \pm 0.05$ \\
K2K 2005 & $\sim 1.3$ & $\mathrm{H}_{2} \mathrm{O}$ & $\frac{\sigma\left(\nu_{\mu} N \pi^{0}\right)}{\sigma(C C)}=0.064 \pm 0.001_{\text {stat. }} \pm 0.007_{\text {sys. }}$ \\
\hline \hline
\end{tabular}

For resonant $\pi^{0}$ production via neutral current interactions, several experimental measurements using $\mathrm{GeV}$ neutrino have been performed in the past 40 years as shown in Table 1.2. Measurements in early dates were performed in bubble chamber experiments: ANL [19,20]. Gargamelle [21], and spark chamber experiments in BNL [27]. The precision of the measurements is at a $20 \%$ level, limited by low statistics. In addition, the results are expressed as the ratio to the charged current (CC) single pion production cross section, which is also poorly known. This is not a useful expression for predicting electron backgrounds in $\nu_{\mu} \rightarrow \nu_{e}$ oscillation searches, since the $\nu_{\mu}$ flux is usually measured by inclusive $\mathrm{CC}$ or CCQE interaction in the long baseline neutrino oscillation experiments such as T2K.

Recently, two experiments published single $\pi^{0}$ production results. Although these experiments measure inclusive (resonant + coherent) pion production, the most of contribution is resonant pion production ( the coherent fraction is less than $20 \%$ ). The K2K collaboration reported the cross section ratio of the $\mathrm{NC} \pi^{0}$ production to total CC events in water with a $1.3 \mathrm{GeV}$ mean neutrino energy beam [28]. Their result of the cross section ratio is consistent with the Monte Carlo (MC) prediction based on the Rein and Sehgal model [18]. MiniBooNE reported the yield and spectral shape of $\pi^{0} \mathrm{~s}$ as a function of $\pi^{0}$ momentum in mineral oil $\left(\mathrm{CH}_{2}\right)$ in neutrino beam of mean neutrino energy $0.7 \mathrm{GeV}[29]$. However, they do not report the cross section or cross section ratio. The total $\mathrm{NC} \pi^{0}$ cross section below $1 \mathrm{GeV}$ has still not been precisely measured yet. This is the main motivation of our measurement.

\section{Coherent Pion Production}

A smaller contribution comes from coherent scattering where the neutrino interacts with the entire nucleus leaving it in the ground state. This means the effective dimensions of space involved in the interactions is large compared with the dimensions of the target nucleus, i.e.,

$$
\frac{1}{|t|}>R
$$


where $t$ and $R$ are the four-momentum transfer to the target nucleus and the radius of the target nucleus, respectively. Because of the small momentum transfer to the target nucleus the outgoing lepton and pion tend to go in the forward direction in the lab frame, and no nuclear breakup occurs. Both charged current (CC) and neutral current (NC) coherent pion productions are possible;

$$
\begin{aligned}
& \nu_{\mu} A \rightarrow \mu^{-} A \pi^{+} \\
& \nu_{\mu} A \rightarrow \nu_{\mu} A \pi^{0}
\end{aligned}
$$

where $A$ is a nucleus.

There exist several theoretical models for coherent pion production. They are categorized into two different types. The first one is built on the basis of Adler's Partially Conserved Axial-vector Current (PCAC) theorem [30], associating the neutrino-nucleus cross section to the pion-nucleus cross section at $Q^{2}=0$, where $Q^{2} \equiv-\left(P_{\ell}-P_{\nu}\right)^{2}$ is the square of the four-momentum transfer, and $P_{\ell}$ and $P_{\nu}$ are the four-momenta of the outgoing lepton and the incoming neutrino, respectively; the extrapolation to $Q^{2} \neq 0$ is done via a propagator term [31-35]. The second one is based on the description of the coherent production of $\Delta$ resonances on nuclei by using a modified $\Delta$-propagator and a distorted wave-function for the pion [36-38].

The model of Rein and Sehgal $[32,35]$, one of the first category, is commonly used in neutrino oscillation experiments. Since the Rein-Sehgal model is used for this thesis, the details are described in Section 4.2.1. Figure 1.5 shows the predicted or calculated cross sections for $\nu_{\mu}{ }^{12} \mathrm{C} \rightarrow \mu^{-} \pi^{+12} \mathrm{C}$ interaction. The solid line and the dashed line represent the Rein and Sehgal model with lepton mass effects [35] and without lepton mass effects [32], respectively. The cross sections predicted by other recent models are also shown in the figure. As shown in the figure, order-of-magnitude variation on the coherent pion production cross section exist among the different models. Therefore, more experimental input on coherent pion production in neutrino-nucleus interactions is needed.

Table 1.3: List of past measurements of coherent pion production. The experimental results are summarized in Figure 1.6

\begin{tabular}{lcccccc}
\hline \hline Experiment & Beam & Reaction & $E_{\nu}(\mathrm{GeV})$ & Target & $\langle A\rangle$ & Reference \\
\hline Aachen-Padova (AP) & $\nu_{\mu} / \bar{\nu}_{\mu}$ & $\mathrm{NC}$ & 2 & $\mathrm{Al}$ & 27 & {$[39]$} \\
Gargamelle (GGM) & $\nu_{\mu} / \bar{\nu}_{\mu}$ & $\mathrm{NC}$ & 3.5 & Freon & 30 & {$[40]$} \\
SKAT & $\nu_{\mu} / \bar{\nu}_{\mu}$ & $\mathrm{CC} / \mathrm{NC}$ & $3-30$ & Freon & 30 & {$[41]$} \\
CHARM & $\nu_{\mu} / \bar{\nu}_{\mu}$ & $\mathrm{NC}$ & $10-160$ & Marble & 20 & {$[42]$} \\
CHARM II & $\nu_{\mu} / \bar{\nu}_{\mu}$ & $\mathrm{CC}$ & $3-300$ & Glass & 20.1 & {$[43]$} \\
BEBC (WA59) & $\nu_{\mu} / \bar{\nu}_{\mu}$ & $\mathrm{CC}$ & $5-150$ & $\mathrm{Ne}$ & 20 & {$[44,45]$} \\
FNAL E632 & $\nu_{\mu} / \bar{\nu}_{\mu}$ & $\mathrm{CC}$ & $10-300$ & $\mathrm{Ne}$ & 20 & {$[46,47]$} \\
K2K & $\nu_{\mu}$ & $\mathrm{CC}$ & 1.3 & $\mathrm{C}$ & 12 & {$[48]$} \\
MiniBooNE & $\nu_{\mu}$ & $\mathrm{NC}$ & 1.2 & $\mathrm{C}$ & 12 & {$[29]$} \\
\hline \hline
\end{tabular}

Coherent pion production has already been the subject of several experimental campaigns. Table 1.3 summarizes the past measurements of coherent pion production. Although there exist positive coherent pion production results at higher energy (3-300 GeV 


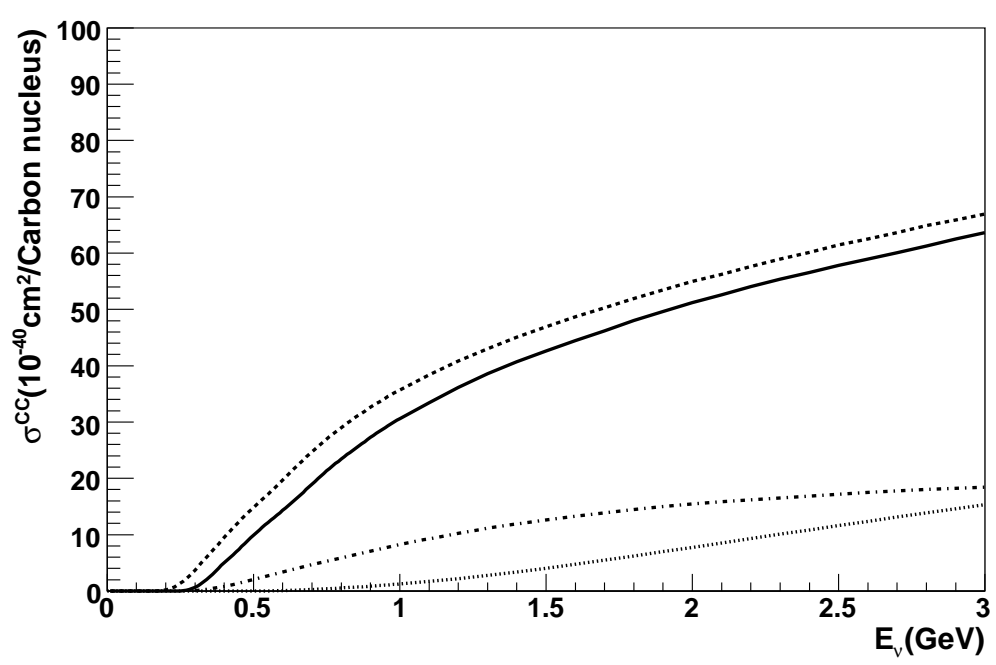

Figure 1.5: Cross section for $\nu_{\mu}{ }^{12} \mathrm{C} \rightarrow \mu^{-} \pi^{+12} \mathrm{C}$ interaction. The solid line represents the Rein and Sehgal model with lepton mass effects [35], the dashed line represents the Rein and Sehgal model without lepton mass effects [32], the dotted line represents the model of Kartavtsev et al. [34], and the dashed-dotted line represents the model of Alvarez-Ruso et al. [38]. The model of Singh et al. [36] gives a cross section similar to the model of Alvarez-Ruso et al.

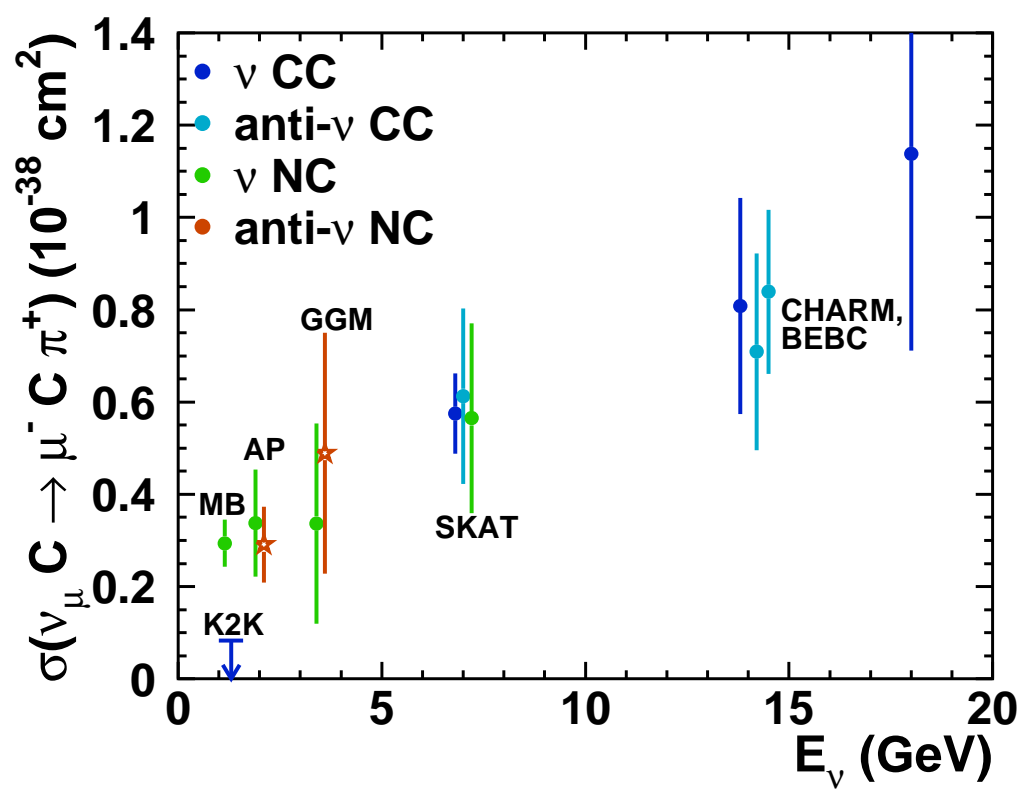

Figure 1.6: Existing experimental results on the coherent pion production cross section $\left(E_{\nu}<20 \mathrm{GeV}\right)$. The results are scaled to the cross section for $\nu_{\mu}{ }^{12} \mathrm{C} \rightarrow \mu^{-} \pi^{+12} \mathrm{C}$ by assuming (a) $\sigma(\mathrm{CC})=2 \sigma(\mathrm{NC})$, (b) $\sigma\left(\nu_{\mu}\right)=\sigma\left(\overline{\nu_{\mu}}\right)$, and (c) $A^{2 / 3}$ dependence. For the $\mathrm{K} 2 \mathrm{~K}$ result, the upper limit on the cross section ratio to total charged current is converted using the predicted value of the total charged current cross section quoted in their paper. Similarly, for the MiniBooNE result, the cross section ratio to all neutral current single neutral pion production is converted using the Rein-Sehgal prediction for resonant pion production. 
neutrino energy) via charged and neutral current interactions, the K2K experiment with a $1.3 \mathrm{GeV}$ wide-band neutrino beam [48] reports null observation of charged current coherent pion production. This result is confirmed by the SciBooNE experiment [49]. Meanwhile, the MiniBooNE Collaboration with similar neutrino energy to K2K observes the neutral current coherent pion production but their observation is $65 \%$ of the Rein and Sehgal prediction. We also show a summary of existing experimental results on the coherent pion production cross section below $20 \mathrm{GeV}$ in Figure 1.6. The Rein and Sehgal model well explains these experimental measurements except for results from the $\mathrm{K} 2 \mathrm{~K}$, SciBooNE and MiniBooNE experiments. From these facts, the coherent pion production have drawn much attention in the neutrino interaction physics community in addition to the importance for the $\nu_{\mu} \rightarrow \nu_{e}$ oscillation search. Hence, it is interesting to confirm the NC coherent $\pi^{0}$ production.

\subsection{Overview of This Thesis}

In this thesis, we present measurements of the $\mathrm{NC} \pi^{0}$ interaction in polystyrene $\left(\mathrm{C}_{8} \mathrm{H}_{8}\right)$ with mean neutrino energy $0.7 \mathrm{GeV}$. We measure the ratio of the total inclusive $\mathrm{NC} \pi^{0}$ cross section to the total CC cross section and kinematic distributions of the $\pi^{0} \mathrm{~s}$. We also extract the fraction of coherent $\mathrm{NC} \pi^{0}$ events in the inclusive NC $\pi^{0}$ data sample. In these analyses, we define $\mathrm{NC} \pi^{0}$ events to be $\mathrm{NC}$ neutrino interactions with at least one $\pi^{0}$ emitted in the final state from the target nucleus.

The measurement in this thesis is the first high statistic $\mathrm{NC} \pi^{0}$ measurement at the mean neutrino energy below $1 \mathrm{GeV}$. Hence, it helps the $\nu_{\mu} \rightarrow \nu_{e}$ oscillation search, especially for T2K. Our measurements use the fully active scintillating tracker as described in Chapter 3 while the existing high statistic $\pi^{0}$ production measurements are using Cherenkov detectors. Hence, our measurements provide new approach to the $\pi^{0}$ production via neutrino interactions, which is important information for the future developments of $\pi^{0}$ detection and reconstruction techniques. In addition, we could observe the recoil protons via neutrino interactions due to the full activity of the scintillating tracker. Using the information of the recoil proton, we can unambiguously distinguish the coherent pion production from the incoherent pion production since the proton recoil occurs only in the incoherent pion production. For these reasons, the measurements presented in this thesis is very much important for the neutrino physics.

This thesis is organized as follows. Chapter 2 describes the overview of the SciBooNE experiment. In Chapter 3, we describe the experimental setup including the neutrino beamline, the detector configuration, and the data set. In Chapter 4, we describe the Monte Carlo (MC) simulations for the neutrino flux prediction, neutrino interactions with nuclei and the particle transportation in the detector. In Chapter 5, we describe the event selection for the NC $\pi^{0}$ events. Then, in Chapter 6 , we discuss the study of the $\mathrm{NC} \pi^{0}$ production including the measurement of the ratio of the $\mathrm{NC} \pi^{0}$ production to total charged current cross sections, the $\pi^{0}$ kinematic distribution and the coherent pion extraction. The conclusions are given in Chapter 7 . 


\section{Chapter 2}

\section{SciBooNE Experiment}

In this chapter, we describe the overview and physics motivations of the SciBooNE experiment.

\subsection{Overview of SciBooNE}

The SciBar Booster Neutrino Experiment (SciBooNE) [50] is designed for measuring the neutrino-nucleus cross sections around one $\mathrm{GeV}$ region, which is essential for neutrino oscillation experiments such as T2K. We briefly summarize features of the SciBooNE experiment;

\section{- High intensity low energy neutrino beam}

SciBooNE uses the Booster Neutrino Beam (BNB) at Fermi National Accelerator Laboratory (FNAL), which has been used for the MiniBooNE experiment. The BNB can provide a high rate and low energy neutrino beam. In the BNB, an antineutrino beam can be produced by reversing the polarity of the horn current.

\section{- Fully active fine segmented scintillator tracking detector}

SciBooNE uses the Scintillator Bar (SciBar) detector, a fully active fine segmented tracking detector. The SciBar detector was originally developed and used for the K2K experiment [51]. SciBar acts as the neutrino target, and also can detect all charged particles produced by neutrino interactions.

These two main feature give us an efficient opportunity for precise measurements of neutrino cross sections since both are already built and have been operated successfully.

Figure 2.1 shows a schematic drawing of the experimental setup of SciBooNE. The SciBooNE detector is positioned $100 \mathrm{~m}$ downstream from the proton target on the axis of the beam. The MiniBooNE detector is located $440 \mathrm{~m}$ downstream from the SciBooNE detector, exposed by the same neutrino beam. The detector comprises three sub-detectors; the SciBar detector is followed by an electromagnetic calorimeter (EC) and a muon range detector (MRD). Detailed descriptions of the BNB and the SciBooNE detector are given in Chapter 3. 


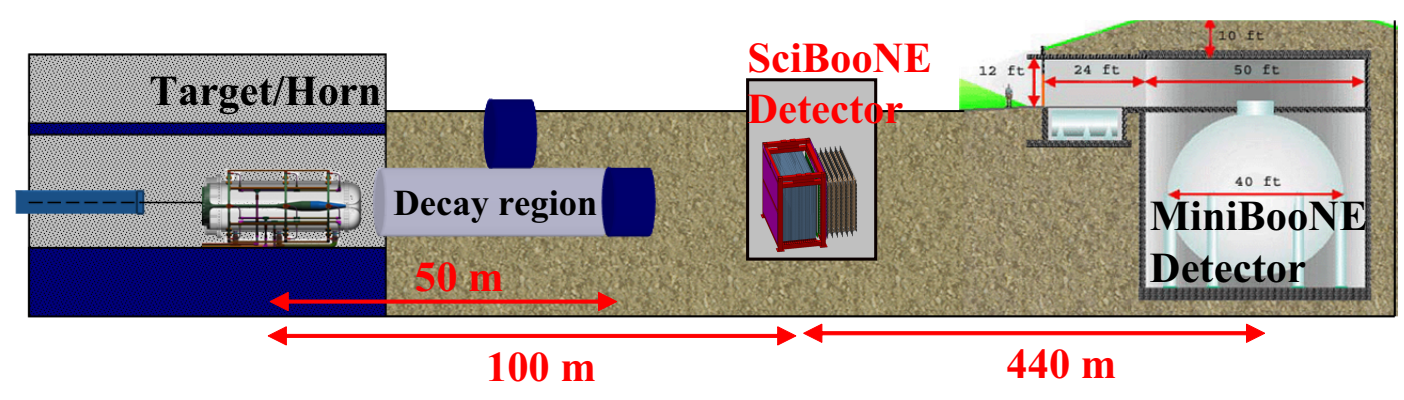

Figure 2.1: Schematic drawing of the experimental setup of SciBooNE.

\subsection{Physics Motivations of SciBooNE}

SciBooNE has three main physics motivations: precise measurements of neutrinonucleus cross sections, measurements of antineutrino-nucleus cross sections, and the BNB neutrino flux measurements.

\subsubsection{Precise Measurements of Neutrino-nucleus Cross Sections}

Figure 2.2 shows the $\nu_{\mu}$ energy spectrum at SciBooNE with those at K2K and T2K. Since the entire range of the T2K energy spectrum is covered within the spectrum of SciBooNE, various neutrino cross section measurements at SciBooNE could help neutrino oscillation studies in $\mathrm{T} 2 \mathrm{~K}$. The measurement of the $\mathrm{NC} \pi^{0}$ production is classified into this category and the primary motivation of the SciBooNE experiment.

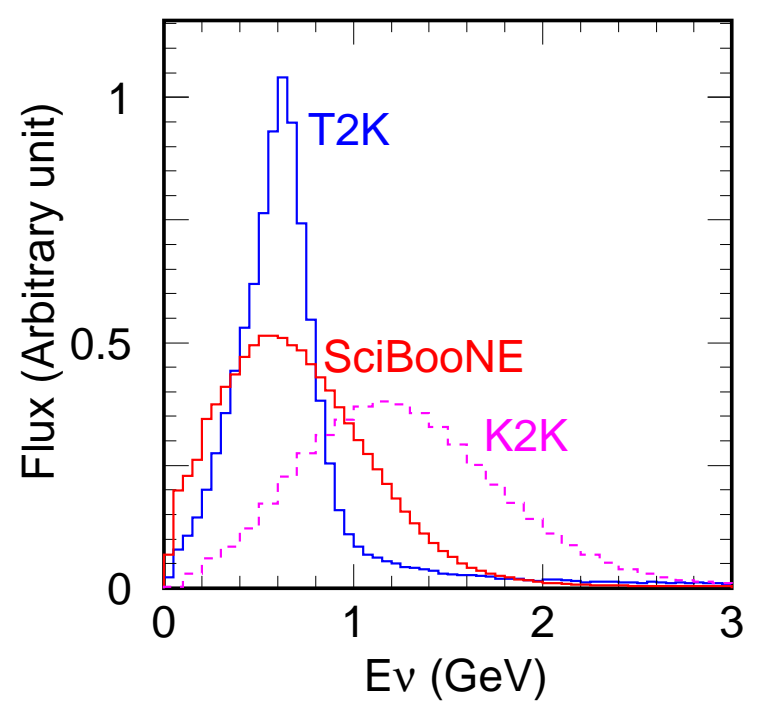

Figure 2.2: Comparison of the muon neutrino energy spectra at $\mathrm{K} 2 \mathrm{~K}, \mathrm{~T} 2 \mathrm{~K}$, and SciBooNE. All curves are normalized to unit area.

\subsubsection{Measurements of Antineutrino-nucleus Cross Sections}

If a non-zero $\theta_{13}$ is found, $\mathrm{T} 2 \mathrm{~K}$ will search for $\mathrm{CP}$ violation in the neutrino sector. It requires oscillation measurements with both neutrino and antineutrino beams. However, the current knowledge of antineutrino cross sections in the few $\mathrm{GeV}$ range is very poor 
with few low statistics measurements. SciBooNE can measure various antineutrino cross sections with high precision.

\subsubsection{BNB Neutrino Flux Measurements}

The $\nu_{\mu}$ flux normalization and energy spectrum measured by SciBooNE can be use for neutrino oscillation searches at MiniBooNE. The sensitivity study of $\nu_{\mu} \rightarrow \nu_{x}$ and $\bar{\nu}_{\mu} \rightarrow \bar{\nu}_{x}$ oscillations with flux normalization and shape systematics [50] indicates the utility of an external measurement of the neutrino flux. In the $\bar{\nu}_{\mu} \rightarrow \bar{\nu}_{x}$ oscillation search, it is crucial to understand the wrong-sign $\left(\nu_{\mu}\right)$ background, and we can extract the normalization and energy spectrum of the background by using the SciBooNE experiment. 


\section{Chapter 3}

\section{Experimental Setup and Data Set}

In this chapter, we describe the experimental setup of the SciBooNE experiment. We also describe the data set used for this analysis.

\subsection{Booster Neutrino Beam}

Fermilab Booster accelerates the protons up to $8 \mathrm{GeV}$ kinetic energy. Selected spills containing approximately $4-5 \times 10^{12}$ protons are extracted and bent toward the BNB target hall. Each spill contains 81 bunches of protons, approximately 6 nsec wide each and $19 \mathrm{nsec}$ apart, for a total spill duration of $1.6 \mu \mathrm{sec}$. The typical beam alignment and divergence, measured by the beam position monitors located near the target, are within 1 $\mathrm{mm}$ and $1 \mathrm{mrad}$ of the nominal target center and axis direction, respectively. The typical beam focusing on target measured by the beam profile monitors is of the order of $1-2 \mathrm{~mm}$ (RMS) in both the horizontal and vertical directions. The number of protons delivered to the BNB target for each spill is measured with a $2 \%$ accuracy using two toroidal current transformers (often referred to as toroid's) located near the target along the beamline. These parameters are well tuned within the experiment requirements.

\subsubsection{Target and Magnetic Focusing Horn}

The primary proton beam smashes a thick beryllium target located in the BNB target hall. Secondary mesons (pions and kaons) are produced by hadronic interactions of the protons with the target. The target is made of seven cylindrical slugs with a radius of $0.51 \mathrm{~cm}$, for a total target length of $71.1 \mathrm{~cm}$, or about 1.7 inelastic interaction lengths. The target is surrounded by a magnetic focusing horn, focusing the positively-charged secondary particles from the target to the direction pointing to the SciBooNE detector. Such positively-charged secondary particles are dominated by charged pions $\left(p i^{+}\right)$producing the neutrino beam via their decay $\left(p i^{+} \rightarrow \mu^{+} \nu_{\mu}\right)$ The focusing is produced by the toroidal magnetic field present in the air volume between the horn's two coaxial conductors made of aluminum alloy. The horn current pulse is approximately a half-sinusoid of amplitude $174 \mathrm{kA}, 143 \mu \mathrm{sec}$ long, synchronized to each beam spill. The polarity of the horn current flow can be (and has been) switched, in order to focus negatively-charged mesons, and therefore to produce an antineutrino beam instead of a neutrino beam. 


\subsubsection{Decay Region and Absorber}

The secondary mesons from the target/horn region are further collimated via passive shielding, and moved to a cylindrical decay region where the secondary mosons can decay into neutrinos. The decay region is filled with air at atmospheric pressure, $50 \mathrm{~m}$ long and $90 \mathrm{~cm}$ in radius. A beam absorber located at the end of the decay region stops hadronic particles and muons, and only a pure neutrino beam pointing toward the detector remains, mostly from $\pi^{+} \rightarrow \mu^{+} \nu_{\mu}$ decays.

\subsection{SciBooNE Detector}

A schematic drawing of the SciBooNE detector is shown in Figure 3.1. The SciBooNE detector consists of three sub-detectors: a fully active and finely segmented scintillator bar tracker (SciBar), an electromagnetic calorimeter (EC) and a muon range detector (MRD). In the following sections, sub-detectors and the data acquisition system are described. The detector coordinate are also described.

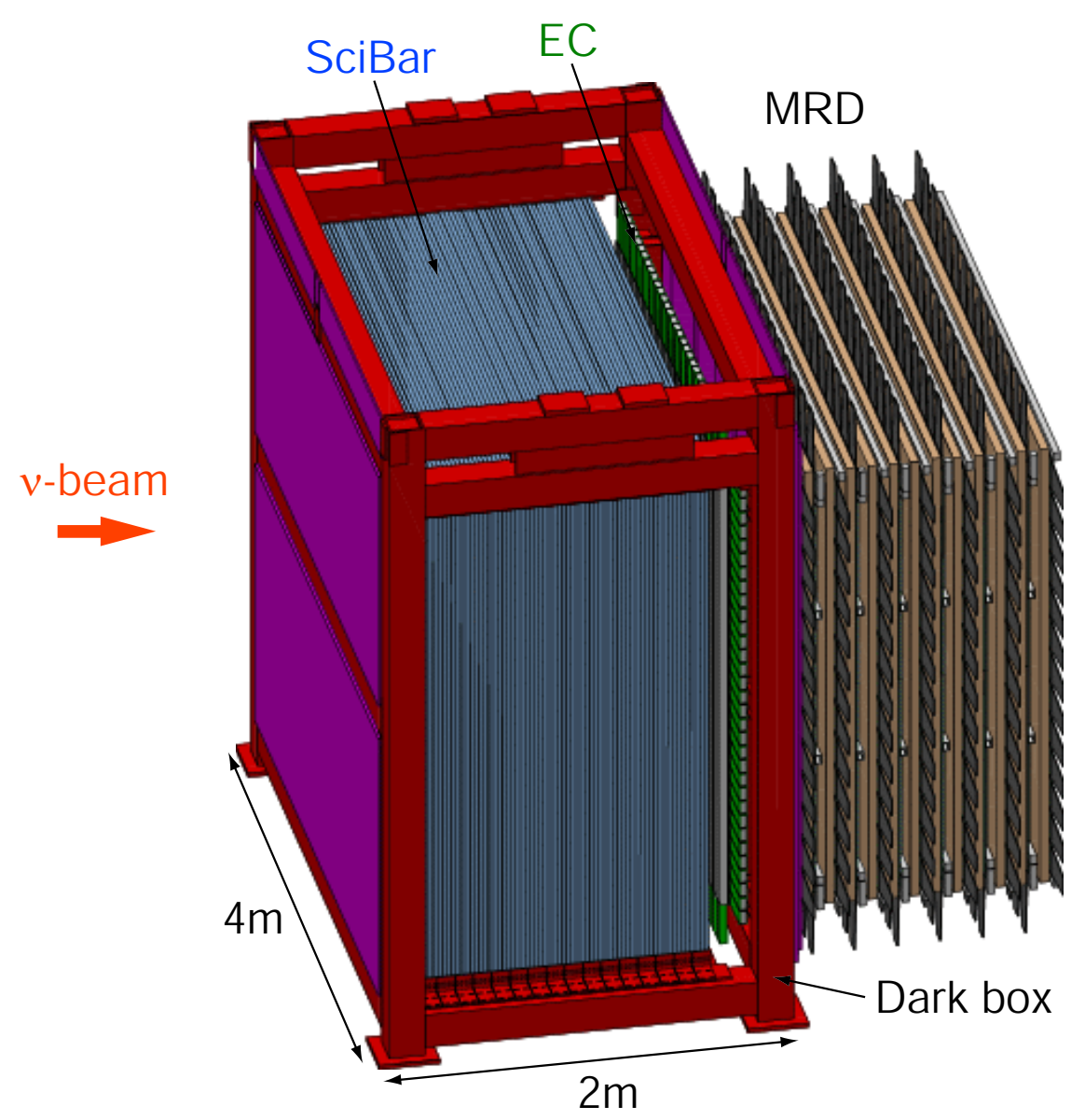

Figure 3.1: Schematic drawing of the SciBooNE detector. 


\subsubsection{Scintillator Bar Tracker (SciBar)}

The SciBar detector is located at the upstream of the other sub-detectors. The most important role of SciBar is to reconstruct the neutrino-nucleus interaction vertex and detect charged particles produced by neutrino interactions. In addition, SciBar can identify particles based on energy deposition per unit length. SciBar was originally designed and built as a near detector for the $\mathrm{K} 2 \mathrm{~K}$ experiment [51]. After $\mathrm{K} 2 \mathrm{~K}$ was completed, the SciBar detector was once disassembled, shipped to FNAL, and then re-built there for SciBooNE.

Figure 3.2 shows a schematic drawing of SciBar. The SciBar detector consists of 14,336 extruded plastic scintillator strips which serve as the target for the neutrino beam as well as the active detection medium. Each strip has dimensions of $1.3 \times 2.5 \times 300 \mathrm{~cm}^{3}$. The scintillators are arranged vertically and horizontally to construct a $3 \times 3 \times 1.7 \mathrm{~m}^{3}$ volume with a total mass of 15 tons. Each strip is read out by a wavelength shifting (WLS) fiber, Kuraray Y11(200)MS attached to a 64-channel multi-anode photomultiplier tube (MA-PMT), Hamamatsu H8804 as shown in Figure 3.3. The hit finding efficiencies evaluated with cosmic ray data are $99.8 \%$ and $99.9 \%$ for the vertical and horizontal planes, respectively. The track finding efficiency for single tracks of $10 \mathrm{~cm}$ or longer is more than $99 \%$. The radiation length of polystyrene (scintillator material) is approximately about $43 \mathrm{~cm}$. Since the length of SciBar along the beam axis is $1.7 \mathrm{~m}, \mathrm{SciBar}$ has the thickness of 4.0 radiation length. Table 3.1 summarizes specifications of the SciBar detector. In the following sections, we describe the readout electronics, the gain monitoring system and the energy calibration in details.

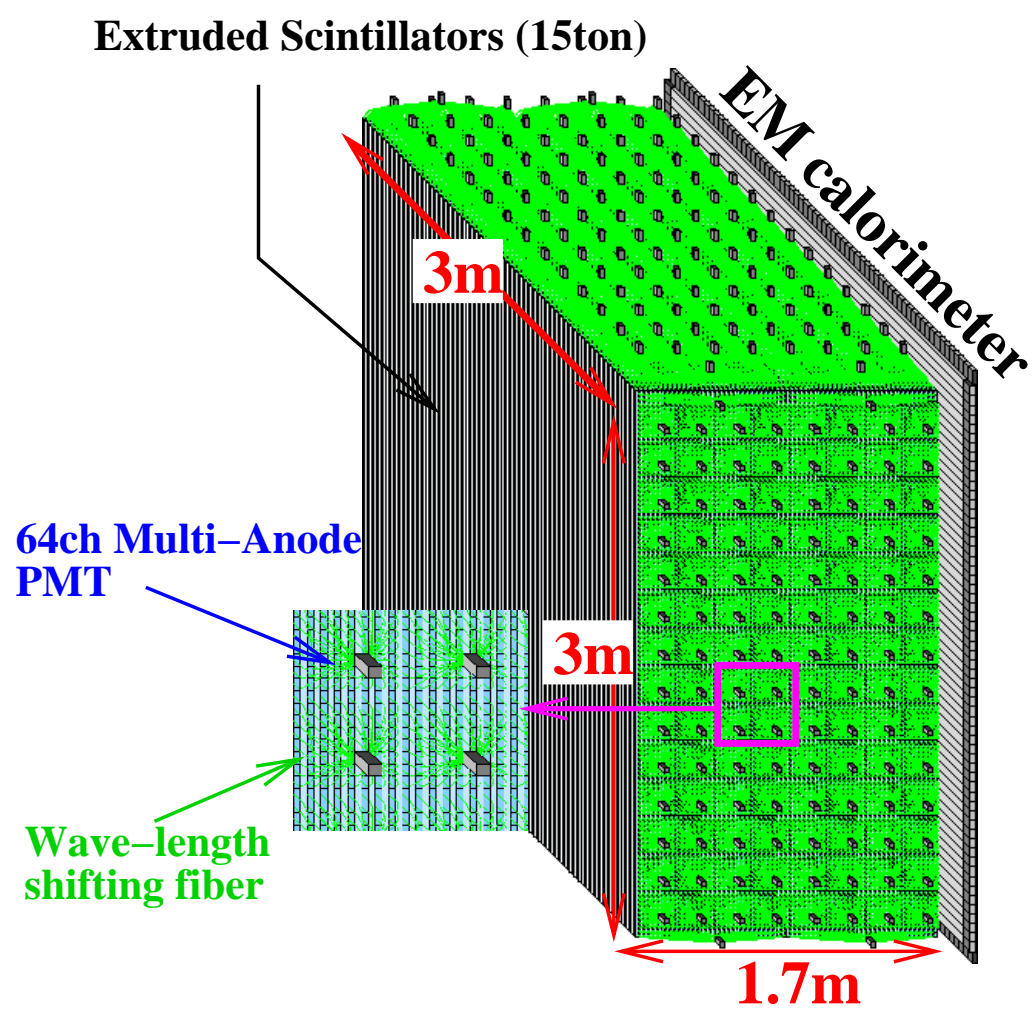

Figure 3.2: Schematic drawing of SciBar. 
Table 3.1: Specifications of the SciBar detector

\begin{tabular}{|c|c|}
\hline \multicolumn{2}{|r|}{ Structure } \\
\hline Dimensions & $3 \mathrm{~m} \times 3 \mathrm{~m} \times 1.7 \mathrm{~m}$ \\
\hline Weight & 15 tons \\
\hline Number of channels & 14,336 \\
\hline \multicolumn{2}{|r|}{ Scintillator } \\
\hline Material & Polystyrene, $\mathrm{PPO}(1 \%), \mathrm{POPOP}(0.03 \%)$ \\
\hline Emission peak wavelength & $420 \mathrm{~nm}$ \\
\hline Reflector material & $\mathrm{TiO}_{2}(15 \%)$ infused in polystyrene \\
\hline Dimensions & $1.3 \mathrm{~cm} \times 2.5 \mathrm{~cm} \times 300 \mathrm{~cm}$ \\
\hline Density & $1.021 \mathrm{~g} / \mathrm{cm}^{3}$ \\
\hline \multicolumn{2}{|r|}{ WLS fiber } \\
\hline Type & Kuraray Y11(200)MS, multi-clad \\
\hline Material & polystyrene(core), acrylic(inner), polyfluor(outer) \\
\hline Refractive index & 1.56 (core), 1.49 (inner), 1.42 (outer) \\
\hline Absorption peak wavelength & $430 \mathrm{~nm}$ \\
\hline Emission peak wavelength & $476 \mathrm{~nm}$ \\
\hline Diameter & $1.5 \mathrm{~mm}$ \\
\hline Attenuation length & 350 cm (typical) \\
\hline \multicolumn{2}{|r|}{ MA-PMT } \\
\hline Model & Hamamatsu H8804 \\
\hline Anode & $8 \times 8$ pixels (pixel size: $2 \times 2 \mathrm{~mm}^{2}$ ) \\
\hline Cathode & Bialkali (Sb-K-Cs) \\
\hline Sensitive wavelength & 300-650 nm (peak: $420 \mathrm{~nm}$ ) \\
\hline Quantum efficiency & $12 \%$ at $\lambda=500 \mathrm{~nm}$ \\
\hline Dynode & Metal channel structure, 12 stages \\
\hline Gain & typical $6 \times 10^{5}$ at $800 \mathrm{~V}$ \\
\hline Response linearity & $\begin{array}{l}\text { within } 10 \% \text { up to } 200 \text { photoelectrons } \\
\text { with the gain of } 6 \times 10^{5}\end{array}$ \\
\hline Crosstalk & $3.15 \%$ (adjacent pixel) \\
\hline \multicolumn{2}{|r|}{ Readout electronics } \\
\hline Number of ADC channels & 14,336 \\
\hline ADC pedestal width & less than 0.3 photoelectron \\
\hline ADC response linearity & $\begin{array}{l}\text { within } 5 \% \text { up to } 300 \text { photoelectrons } \\
\text { with the gain of } 5 \times 10^{5}\end{array}$ \\
\hline Number of TDC channels & 448 \\
\hline TDC resolution & $0.78 \mathrm{nsec}$ \\
\hline TDC full range & $50 \mu \mathrm{sec}$ \\
\hline
\end{tabular}




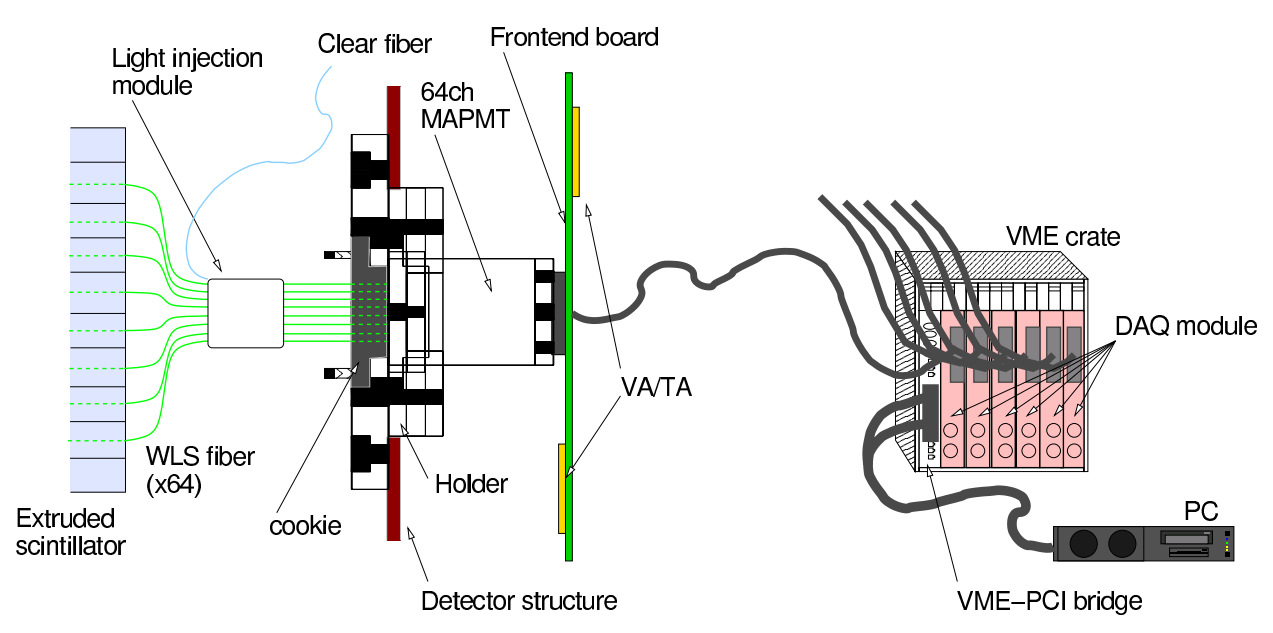

Figure 3.3: Schematic drawing of the SciBar readout system.

\section{Readout Electronics}

The readout electronics system consists of a front-end electronics board (FEB) attached to each MA-PMT and a back-end VME module [52]. Figure 3.4 shows the picture of the FEB. On the FEB, a combination of VA and TA ASICs (IDEAS VA32HDR11 and TA32CG) is employed to multiplex pulse-height information from each anode of the MAPMT and to make a fast-triggering signal. The VA has a 32-channel preamplifier-shaper circuit with a multiplexer. The slow shaper shapes the output with a peaking time of $1.2 \mu \mathrm{sec}$. The signal from each VA slow shaper is sampled at the time of an external hold request, and the result is passed to the multiplexer. The signal after preamplification in the VA is also sent to a fast shaper in the TA with a peaking time of 80 nsec. A logical "OR" of 32 channels is sent out from the TA. The intrinsic time jitter of the discriminated output is less than 1 ns. The TA signal is sent to a 64 -channel multi-hit time-to-digital converter (AMT [53]) and recorded as timing information. Each FEB has two packages of VA/TA, processing 64-channel charge information and two-channel timing information for each MA-PMT. The back-end VME module, called the DAQ board, is developed as a standard VME-9U board. Figure 3.5 shows the picture of the DAQ board. Each DAQ board controls the readout of eight FEBs, and thus 28 DAQ boards are used in total. We use four VME-9U crates for all 28 DAQ boards ( 7 boards per 1 crate). Each of the eight channels in the single DAQ board has line drivers to control the VA and TA ASICs on FEB and a 12-bit flash analog-to-digital converter (ADC) to digitize the multiplexed analog signal from the FEB with a 1-MHz readout clock.

In the SciBooNE operation, the TA signal is used to generate the hold request for sampling the signal of each VA slow shaper. In fact, the DAQ board receives the TA signal from the FEB and generate the hold request signal back to the FEB with $1.2 \mu \mathrm{s}$ delay. By doing this, the signal from the VA slow shaper is always sampled at its peaking time. Therefore, the recorded charge information does not depend on when the neutrino interactions happen during a total spill duration of $1.6 \mu \mathrm{sec}$. The timing sequence related to the TA, VA and hold request is shown in Figure 3.6.

The TA signal is also sent to a cosmic-ray trigger board. The board is a general purpose logic board powered by an FPGA, and programmed to generate a signal when 
a cosmic-ray penetrates almost all the layers of SciBar. The signal is used to record the cosmic-ray events which are useful to the calibration of SciBar and EC.

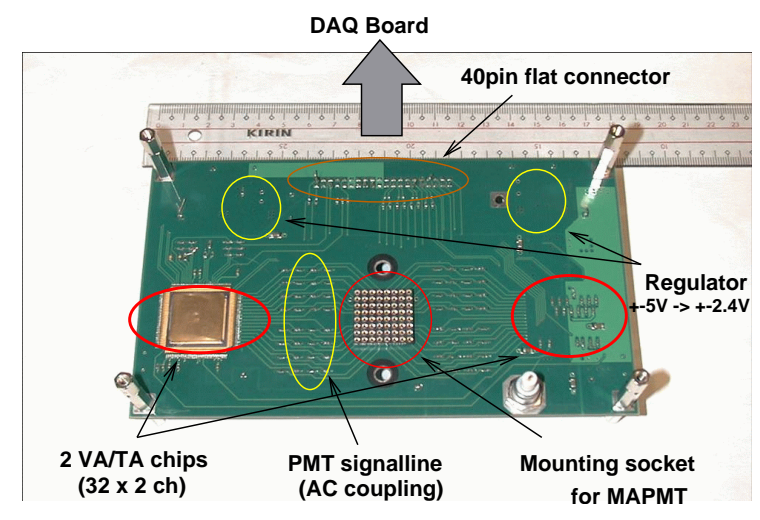

Figure 3.4: Picture of a front-end board.

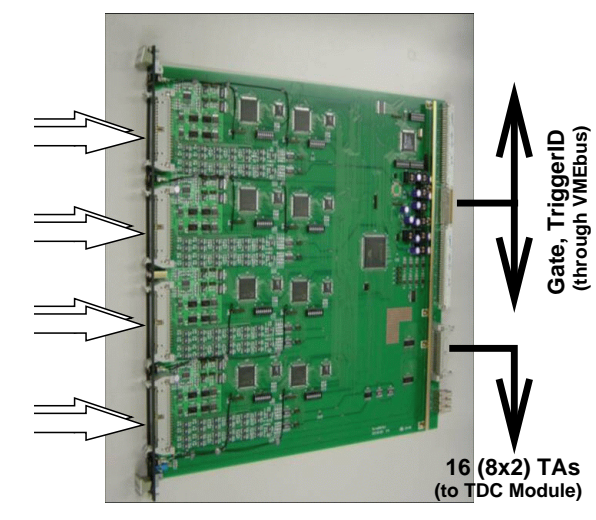

Figure 3.5: Picture of a DAQ board.

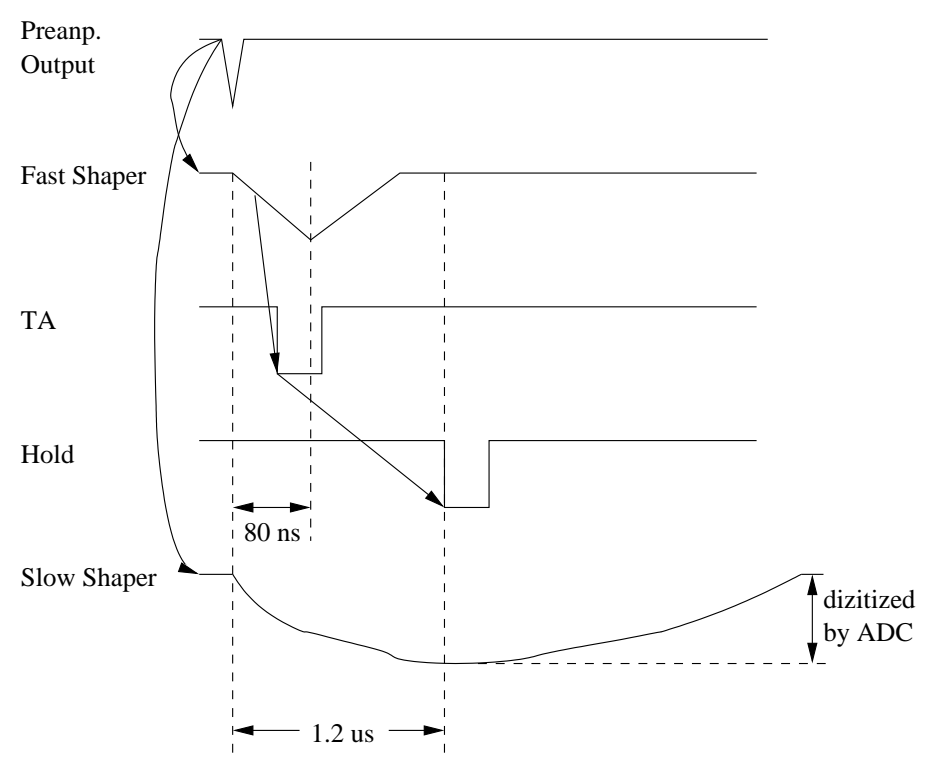

Figure 3.6: The timing sequence of the SciBar electronics

\section{Gain Monitoring System}

The gain stability of the MA-PMTs is ensured within $\pm 2 \%$ by a custom-made monitoring system using light-emitting diodes (LEDs) [54]. The system consists of four sets of light sources, PIN photo-diodes, and clear fiber bundles. A schematic drawing of the SciBar gain monitoring system are shown in Figure 3.7. The pulsed light from each LED is carried to 56 MA-PMTs through 56 clear fibers. A white cylinder, called a Light Injection Module, is attached to the WLS fiber bundle in order to illuminate 64 WLS fibers uniformly. The LED light is absorbed by the WLS fibers, and then emitted light from the WLS fiber is carried to each channel of the MA-PMT. 


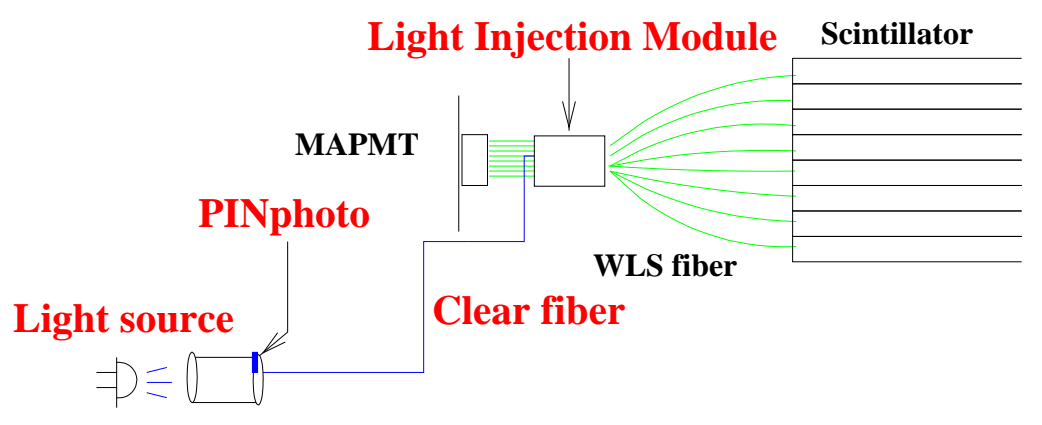

Figure 3.7: Schematic drawing of the SciBar gain monitoring system.

\section{Energy Scale Calibration}

The energy scale for each channel is calibrated with cosmic-ray muons. The number of photoelectrons for cosmic-ray muons for a typical channel are shown in Figure 3.8. The path length of the particle inside the scintillator strip and the light attenuation in the WLS fiber are corrected. The averaged light yield of a minimum ionizing particle is measured to be approximately 20 p.e. per $1.3 \mathrm{~cm}$ path length. The energy calibration constant which converts the number of photoelectrons to the visible energy is measured for each channel. Figure 3.9 shows the energy calibration constants for all channels. The averaged value is 8.1 p.e./MeV, and the channel-by-channel variation is about $20 \%$.

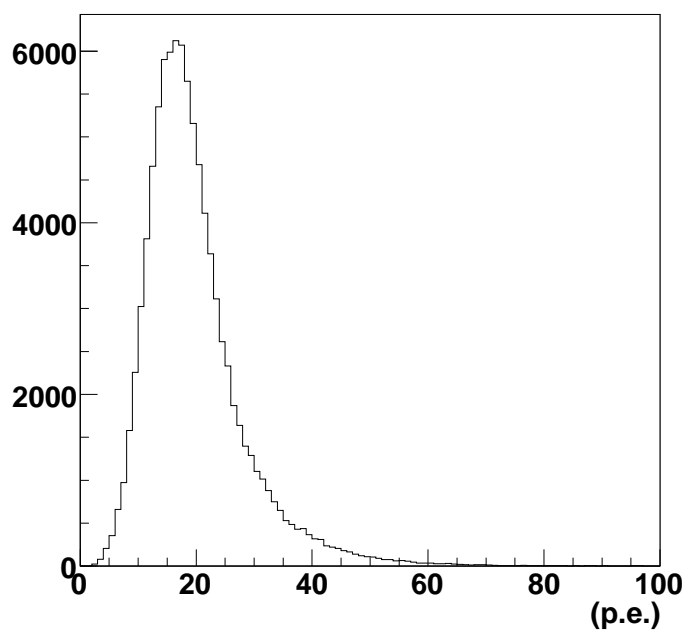

Figure 3.8: Number of photoelectrons for cosmic-ray muons for a typical channel.

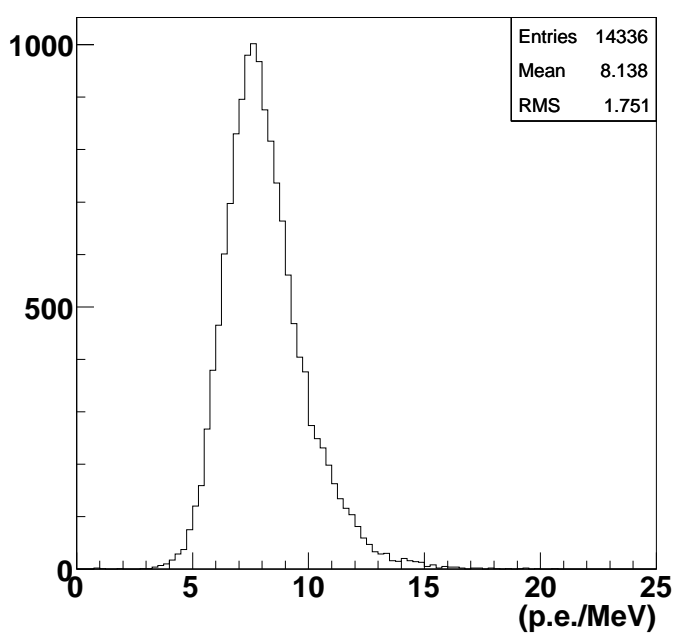

Figure 3.9: Energy calibration constants for all channels.

\subsubsection{Electromagnetic Calorimeter (EC)}

EC is a "spaghetti" type electromagnetic calorimeter, installed downstream of SciBar, and is designed to measure the electron neutrino contamination in the beam and identify 
photons from $\pi^{0}$ decay. The calorimeter modules were originally built for the CHORUS experiment at CERN [55] and later used in HARP and then in K2K.

The calorimeter consists of modules of dimensions $262 \times 8.4 \times 4.2 \mathrm{~cm}^{3}$. The modules construct one vertical and one horizontal plane, and each plane has 32 modules. An active area of $2.7 \times 2.6 \mathrm{~m}^{2}$ is covered by the planes. The EC has a thickness of 11 radiation lengths along the beam direction.

Each module is made of a stack of 21 lead sheets and 740 scintillating fibers. The $1 \mathrm{~mm}$ diameter scintillating fibers, Kuraray SCSF81, are embedded in the grooves on $1.9 \mathrm{~mm}$ thick lead sheets. The stack is held together by a welded steel case. At each end of the module, fibers are grouped into two bundles, and each bundle is connected to a Plexiglas light guide. The light guide is attached to 1 inch PMT, Hamamatsu R1335/SM, with a special green-extended photocathode. A typical gain is $2 \times 10^{6}$ at the operation voltage of $1600 \mathrm{~V}$. In total, 256 PMTs are used in the EC. The attenuation length of the fiber is measured to be approximately $400 \mathrm{~cm}$ by using cosmic-ray muons. The readout system consists of eight 32-channel 12-bit QDC (Charge-to-Digital Converter) modules, CAEN V792. One VME-6U crate is used for QDC readout. The energy resolution for electrons was measured to be $14 \% / \sqrt{E(\mathrm{GeV})}$ in a test beam [55].

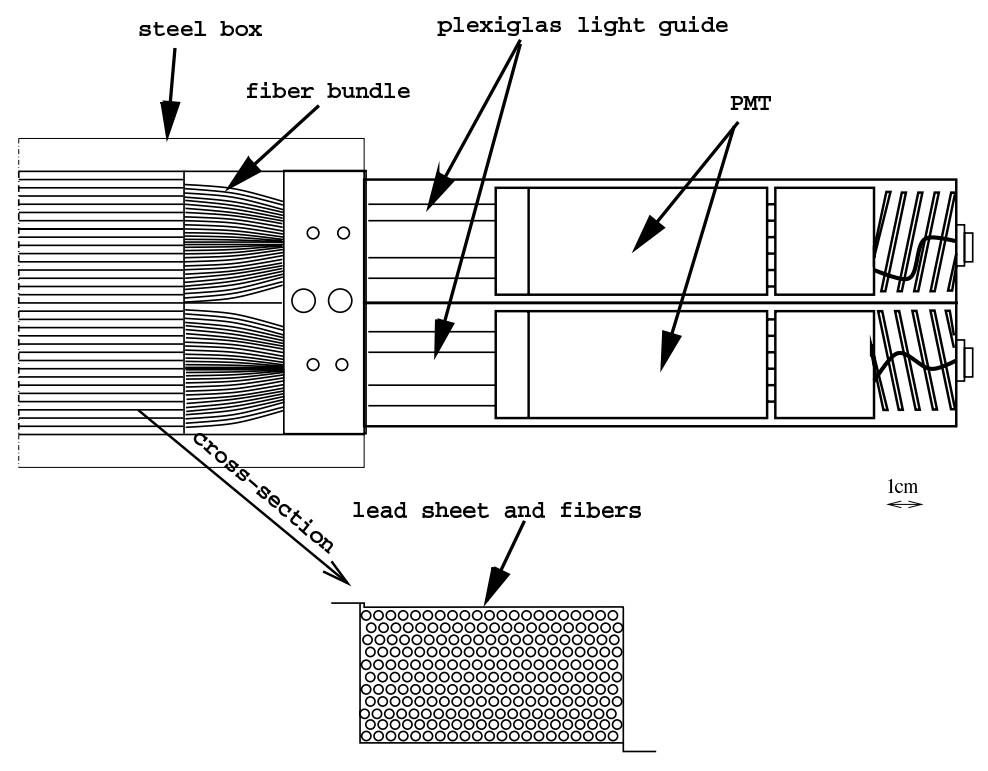

Figure 3.10: Schematic drawing of the EC module.

\subsubsection{Muon Range Detector (MRD)}

The MRD detector is installed downstream of the EC and is designed to measure the momentum of muons produced by charged-current neutrino interactions up to $1.2 \mathrm{GeV} / c$ using the range measurement. The MRD was constructed for SciBooNE at FNAL, primarily by using out of parts recycled from past FNAL experiments.

The MRD consists of 12 iron plates and 13 alternating horizontal and vertical scintillator planes. Each iron plate is 2 inch thick, and covers an area of $274 \times 305 \mathrm{~cm}^{2}$. The total mass of absorber material is approximately 48 tons. The iron plates are sandwiched between scintillator planes. Each scintillator plane consists of $20 \mathrm{~cm}$ wide, $6 \mathrm{~mm}$ thick 
scintillator paddles. Each vertical scintillator plane is comprised of $138 \mathrm{~cm}$ long paddles, arranged in a $2 \times 15$ array to have an active area of $276 \times 300 \mathrm{~cm}^{2}$. On the other hand, each horizontal scintillator plane consists of $155 \mathrm{~cm}$ long paddles, arranged in a $13 \times 2$ array to have an active area of $260 \times 310 \mathrm{~cm}^{2}$. In total, 362 paddles are used in the MRD.

The scintillator paddles are read out by five types of 2 inch PMTs; the vertical planes consist of Hamamatsu 2154-05 and RCA 6342A PMTs, the horizontal planes consist of EMI 9954KB, EMI 9839b and 9939b PMTs. Charge and timing information from each PMT are recorded. The readout electronics system consists of LeCroy 4300B ADCs and LeCroy 3377 TDCs. We use 13 TDCs and 26 ADCs housed in three CAMAC crates. The timing resolution and full range are $0.5 \mathrm{~ns}$ and $32 \mu \mathrm{sec}$, respectively. The energy threshold for TDC hits is approximately $250 \mathrm{keV}$ which corresponds to $20 \%$ of the signal of a minimum ionizing particle. The single noise rate is typically $100 \mathrm{~Hz}$ except for RCA 6342A PMTs which are noisy (up to $10^{4} \mathrm{~Hz}$ ) Hit finding efficiency was continuously monitored by using cosmic ray data taken between beam spills. The average hit finding efficiency is measured to be $99 \%$.

Table 3.2: Specifications of the MRD detector.

\begin{tabular}{ll}
\hline \hline & \multicolumn{1}{c}{ Iron plate } \\
\hline Number of plates & 12 \\
Dimensions & $274 \times 305 \mathrm{~cm}^{2}, 2$ inch thickness \\
Density & $7.841 \mathrm{~g} / \mathrm{cm}^{3}$ \\
\hline & Scintillator plane \\
\hline Number of planes & 13 \\
Segmentation & $2 \times 15$ (vertical), $13 \times 2$ (horizontal) \\
Dimensions of a counter & thickness: $6 \mathrm{~mm}$, width: $20 \mathrm{~cm}$ \\
& length: $138 \mathrm{~cm}$ (vertical), $155 \mathrm{~cm}$ (horizontal) \\
\hline & \multicolumn{1}{c}{ PMT } \\
\hline Model & EMI $9954 \mathrm{~KB}, 9839 \mathrm{~b}$ and $9939 \mathrm{~b}$ (horizontal) \\
& Readout electronics \\
\hline Number of channels & 362 \\
Model & LeCroy $4300 \mathrm{~B}(\mathrm{ADC})$, Lecroy 3377 (TDC) \\
TDC resolution & 0.5 nsec \\
TDC full range & $32 \mu \mathrm{sec}$ \\
\hline \hline
\end{tabular}

\subsubsection{Trigger and Data Acquisition (DAQ) System}

We describe the SciBooNE trigger and DAQ system in this section.

\section{Beam Cycle}

Each spill of the neutrino beam to SciBooNE is synchronized to the $15 \mathrm{~Hz}$ Booster clock. However, not all counts of the Booster clock are occupied by the spills to SciBooNE as shown in Figure 3.11. In general, the neutrino beam comes to SciBooNE with several 


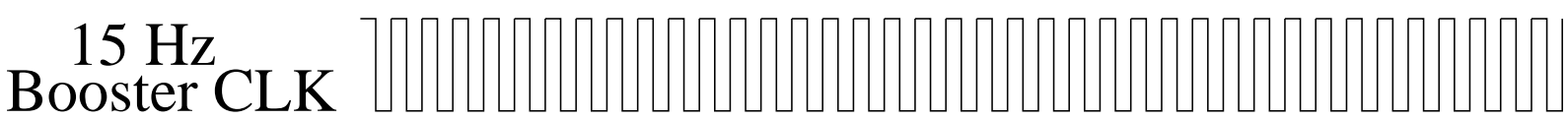 Beam to SciBooNE

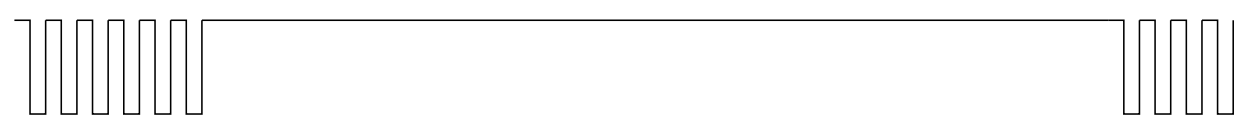 \\ One beam cycle $\sim 2$ second}

Figure 3.11: SciBooNE's beam cycle

continuous spills (up to 10 spills). Then, there is no beam period for more than 1.5 second. Since such several continuous spills and no beam period come to SciBar by turns, we call this repetition "beam cycle". We need to take calibration data during no beam period and stop taking calibration data before the spills come to SciBooNE. However, the number of continuous spills and no beam period change depending on the operation modes of the accelerator. Hence, the ways to generate beam triggers and calibration triggers are not straightforward.

Table 3.3: Tevetron clocks (TCLKs) used for SciBooNE. The $\$ 10, \$ 11, \$ 12$ and $\$ 1 \mathrm{D}$ are synchronized to the $\$ 0 \mathrm{C}$, which is $15 \mathrm{~Hz}$ Booster clock

\begin{tabular}{c|c}
\hline \hline TCLK code & description \\
\hline$\$ 0 \mathrm{C}$ & $15 \mathrm{~Hz}$ Booster clock \\
$\$ 10$ & beam in Booster is accelerated and extracted somewhere \\
$\$ 11$ & no beam in Booster \\
$\$ 12$ & generated twice at the beginning of the beam cycle \\
$\$ 1 \mathrm{D}$ & beam in Booster is accelerated and extracted to SciBooNE \\
\hline $1 \mathrm{~F}$ & Booster kicker timing for extraction \\
\hline \hline
\end{tabular}

To generate beam triggers and calibration triggers at the proper timing, we use several timing signals from the accelerator, called Tevetron clock (TCLK). TCLK is used to transmit important accelerator timing information to all major systems throughout the accelerator complex. Up to 256 unique events or timing markers may be encoded onto TCLK. Each TCLK is expressed by two digit hexadecimal number $(\$ 00-\$ F F)$. The TCLKs related to SciBooNE are $\$ 0 \mathrm{C}, \$ 10, \$ 11, \$ 12, \$ 1 \mathrm{D}$ and $\$ 1 \mathrm{~F}$. We show the descriptions about these TCLKs in Table 3.3. Figure 3.12 shows the timing sequence of TCLKs and the SciBooNE triggers. The $\$ 0 \mathrm{C}$ is the $15 \mathrm{~Hz}$ Booster clock. The other TCLKs listed above are synchronized with $\$ 0 \mathrm{C}$. At the beginning of the beam cycle, two $\$ 12$ s are generated. After the two $\$ 12 \mathrm{~s}$, several $\$ 10$ s are generated. The $\$ 10$ s let us know that the beam in Booster is accelerated and extracted. In case of the beam for the Booster neutrino beamline, $\$ 1 D$ s are also generated with $\$ 10$. After the train of the $\$ 10$ s, several $\$ 11$ s are generated. During $\$ 11 \mathrm{~s}$ are generated, the beam is not in Booster. After the train $\$ 11 \mathrm{~s}$, the two $\$ 12 \mathrm{~s}$ are generated again. This is how the beam cycle proceeds. Although the numbers of $\$ 10$ s, $\$ 1 \mathrm{Ds}$ and $\$ 11$ are not fixed permanently, typical period for one beam 
cycle is about $2 \mathrm{sec}$. We use the first one of two $\$ 12$ to enable beam triggers and disable the calibration triggers. By doing this, we disabled the calibration triggers by at least $2 \times 1 / 15 \mathrm{~Hz}=133 \mathrm{~ms}$ earlier than the next coming beam. This time is much longer than the DAQ deadtime described later $(20 \mathrm{~ms})$. We use the first one of the $\$ 11$ 's train to disable the beam triggers and enable calibration triggers. By doing this, we ensure that the beam trigger is never missed due to the calibration trigger. We use the logical AND of $\$ 1 \mathrm{~F}$ and $\$ 1 \mathrm{D}$ for the beam triggers, where the $\$ 1 \mathrm{~F}$ is the kicker timing for the beam extraction. This is because the $\$ 1 \mathrm{~F}$ has better timing resolution (100 ns) than $\$ 1 \mathrm{D}$ does. For practical purpose, one trigger is generated at the same time when the calibration triggers are enabled by $\$ 11$. We call such triggers off-beam triggers. One off-beam trigger is generated per one beam cycle by definition. Since the beam trigger and off-beam trigger is commonly used by each component of the SciBooNE detectors, we call them global triggers.

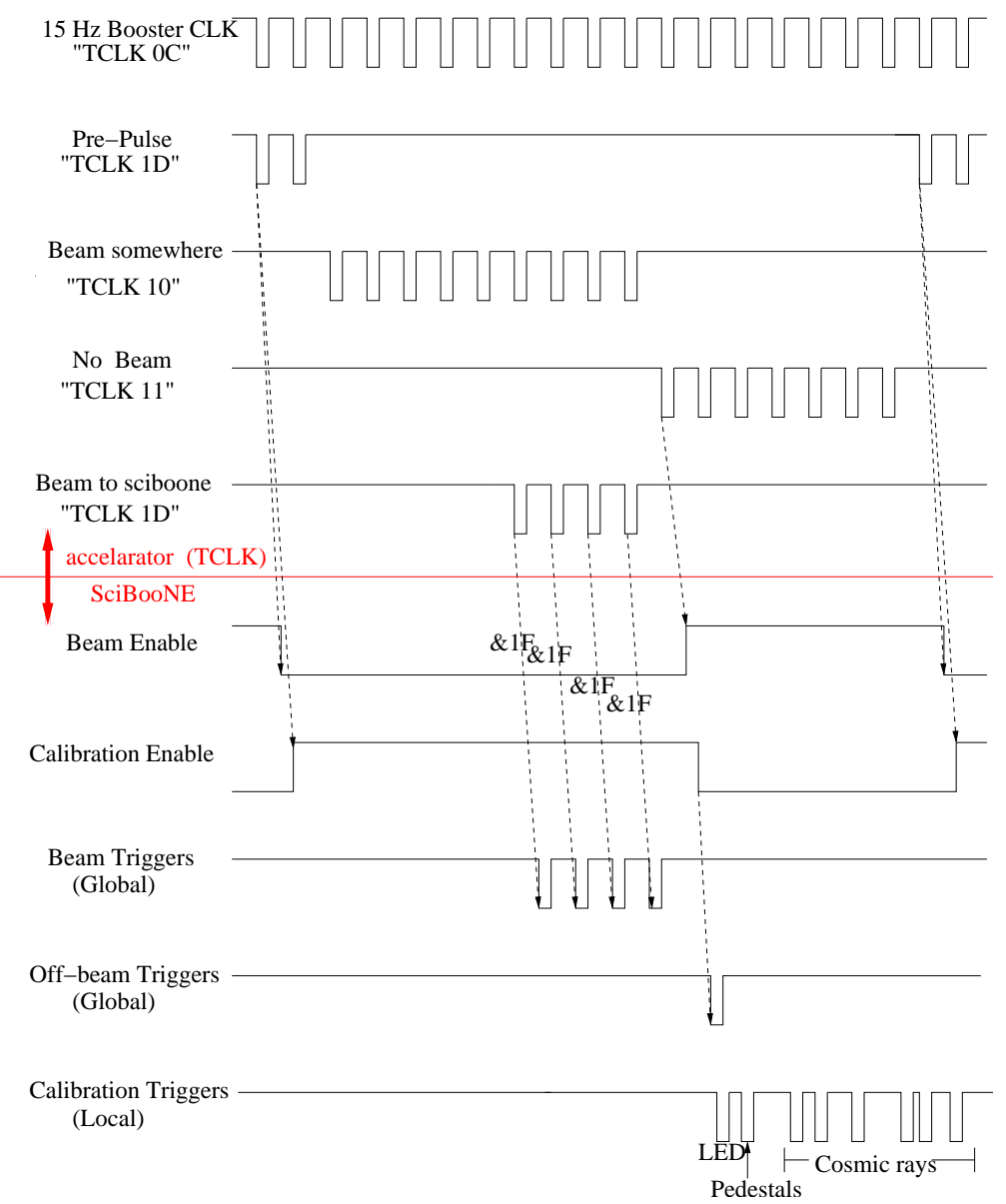

Figure 3.12: The relation between TCLK and the SciBooNE trigger signals

\section{The SciBooNE Trigger}

The beam trigger (logical AND of $\$ 1 \mathrm{D}$ and $\$ 1 \mathrm{~F}$ ) is distributed to all three detectors and used for the ADC gates and reference timings for TDCs. The beam trigger is also distributed to the PCI module called the Time and Frequency Processor, Symetricom 
bc637 PCI (GPS card). This GPS card is used for recording the GPS time for each beam trigger. Once the beam trigger condition is set, all sub-detector systems read out all channels irrespective of hit occupancy (i.e. whether or not a neutrino interaction occurred), ensuring unbiased neutrino data.

SciBar and EC have common calibration triggers, which are the LED, pedestal and cosmic trigger. The LED trigger is for the gain monitor system of SciBar. Since no such gain monitor system in EC, the LED triggers are just pedestal triggers for EC. Meanwhile, MRD generates the pedestal and cosmic trigger independently from SciBar (EC). Therefore, the total numbers of triggers including beam triggers and calibration triggers are different between SciBar (EC) and MRD. We refer to such numbers as numbers of local triggers, hereafter.

\section{Data Stream}

Figure 3.13 shows the schematic drawings of SciBooNE DAQ system. Each VME/CAMAC crate is controlled by single front-end Linux computer. In terms of the DAQ system, SciBar and the EC are treated as one component. For SciBar and the EC, the collected data from each front-end computer are sent to the SciBar-EC local event builder via Ethernet. The local event builder assembles the data and build an event fragment and passes those fragments to a global event builder. For the MRD, the collected data from each front-end computer are directly sent to the global event builder.

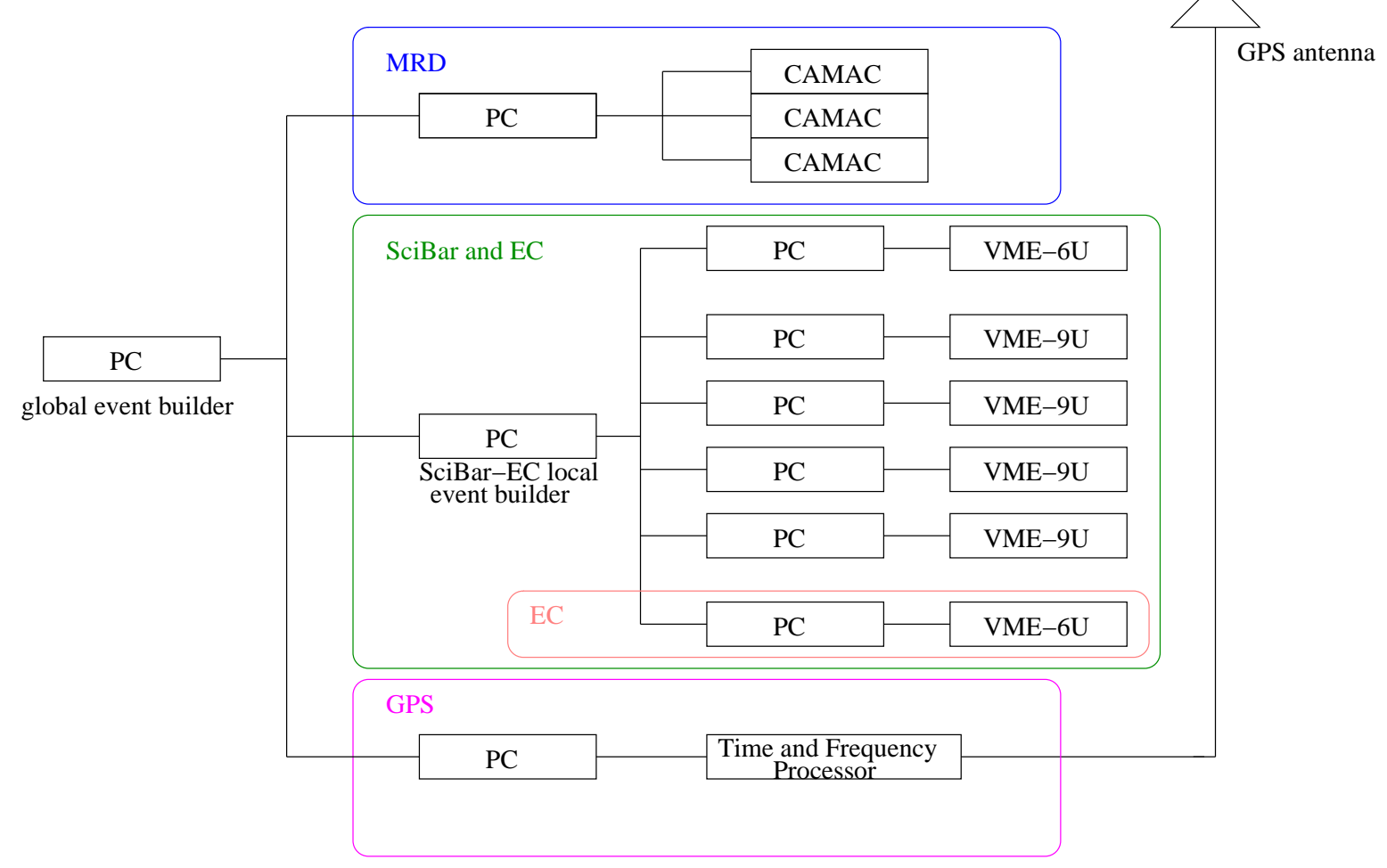

Figure 3.13: Schematic drawing of the SciBooNE DAQ system 


\section{Event Synchronization}

For the event synchronization of the SciBooNE detectors, we use two TRG modules, which are used to record the number of triggers and distribute it. The TRG modules were initially developed for the Super-Kamiokande experiment. One TRG module is located at the VME-6U crate in SciBar. This records the number of local triggers received by SciBar (EC) and distribute it to other VME crates in SciBar (EC). The SciBar-EC local event builder checks the consistency of the numbers from all crates before sending the global event builder. The other TRG module is located at the VME-6U crate connected to the computer with the GPS card. This records the number of global triggers and distribute SciBar and MRD. The global event builder checks consistency the numbers from all components before writing data to the disk. All electronics of the SciBooNE detectors are automatically restarted if the global or SciBar-EC local event builder find the inconsistency between the numbers.

We also need to synchronize the detector information to the accelerator information. The accelerator information is provided by FNAL ACNET (Accelerator Control NETwork). The ACNET DAQ stream is independent from the detector DAQ stream, and the beam and detector information is merged at offline using the GPS time stamps.

\section{Performance}

The typical readout times of SciBar (EC), MRD and GPS are about $17 \mathrm{~ms}$ (less than $1 \mathrm{~ms}$ for only EC),20 ms and less than $1 \mathrm{~ms}$, respectively, for one trigger. The SciBooNE system does not accept the next trigger until all data from each component are read out. As a result, MRD restricts the maximum readout speed $(50 \mathrm{~Hz})$. The spill of Booster Neutrino Beam is always synchronized with $15 \mathrm{~Hz}$ Booster clock. The readout speed (50 $\mathrm{Hz}$ ) is fast enough to take every beam spill. The probability of missing a beam spill due to the readout time is less than $0.002 \%$. The data size of one trigger is about $38 \mathrm{kB}$. It means that the maximum DAQ speed is $1.9 \mathrm{MB}$ per second $(50(\mathrm{~Hz}) \times 38(\mathrm{kB}))$.

\subsubsection{Detector Coordinate}

An event display of a typical muon neutrino event candidate is shown in Figure 3.14. SciBooNE uses a right-handed Cartesian coordinate system in which the $z$ axis is the beam direction and the $y$ axis is the vertical upward direction. Thus, the $x$ axis is the horizontal direction perpendicular to the beam direction. The origin is located on the most upstream surface of SciBar in the $z$ dimension, and at the center of the SciBar scintillator plane in the $x$ and $y$ dimensions. Since each sub-detector is read out both vertically and horizontally, two views are defined; the top view ( $z-x$ projection) and the side view (z-y projection).

\subsection{Data Set}

The SciBooNE experiment took data from June 2007 until August 2008. The datataking is divided into three periods depending on the polarity of the horn: Run-1 (Antineutrino mode), Run-2 (Neutrino mode), and Run-3 (Antineutrino mode). In this chapter, 

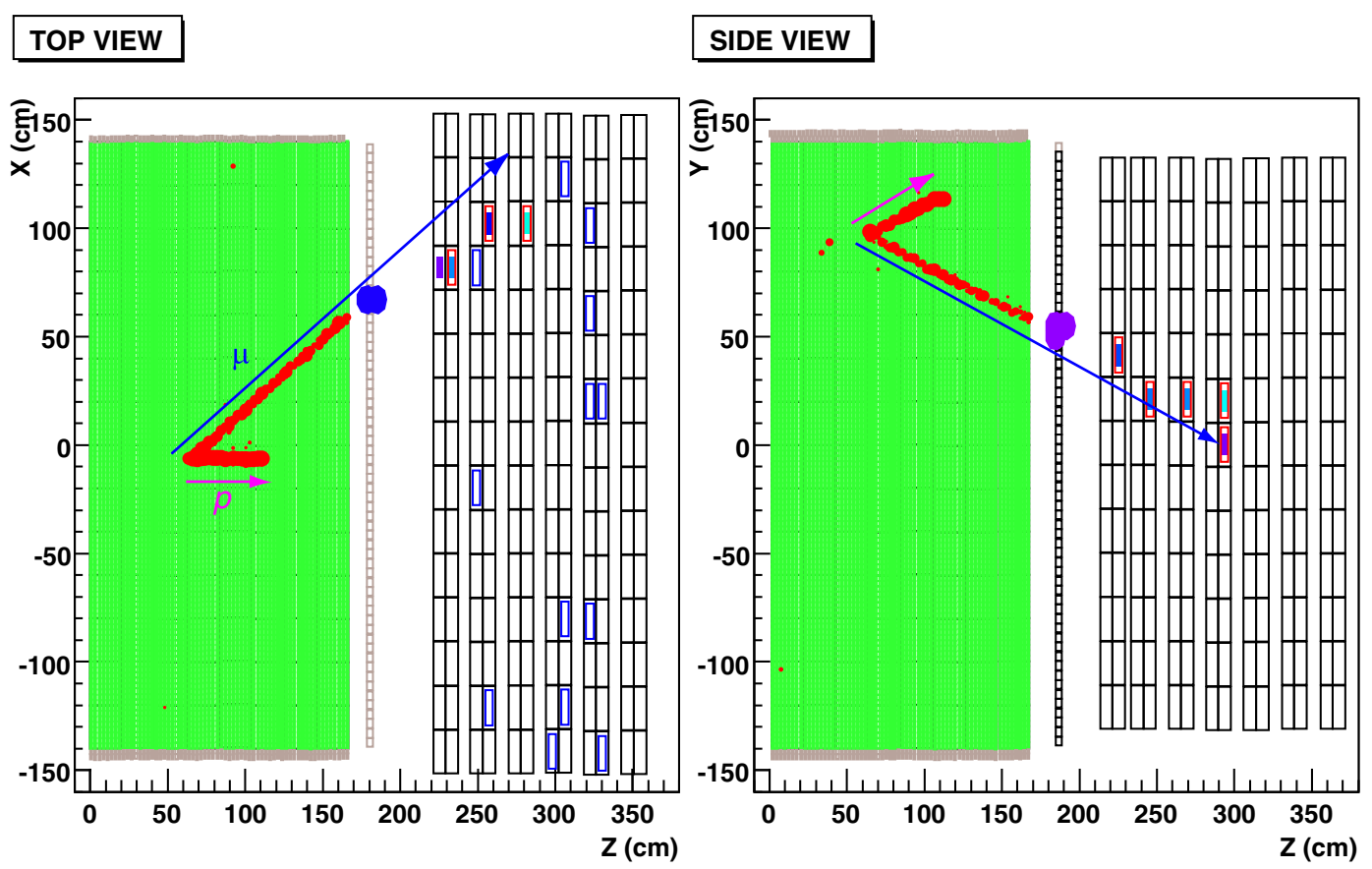

Figure 3.14: Event display of a typical muon neutrino charged current quasi-elastic scattering $\left(\nu_{\mu} n \rightarrow \mu^{-} p\right)$ candidate event by muon neutrinos in SciBooNE. Circles on SciBar indicate $\mathrm{ADC}$ hits, and the area of the circle is proportional to the energy deposition in the scintillator strip. Framed boxes on the MRD indicate TDC hits in the beam-on timing (red) and beam-off timing (blue) windows. Filled boxes on the MRD show ADC hits in the beam-on timing window.

we describe data quality cuts which are applied before any physics analyses, and then we summarize data set used in these analyses.

\subsubsection{Data Quality Cuts}

Only spills that satisfy certain data quality cuts are used for physics analyses. The purpose of the data quality cuts is to ensure that the experimental apparatus is functioning properly. This includes both the beam and detector.

For the beam quality check, the beam-line monitors and the GPS system are used. The detailed selection criteria is found in [56]. Overall, beam quality cuts reject less than $1 \%$ of total number of protons on target accumulated during the run.

For the detector quality check, approximately $1-2 \%$ of beam spills were lost due to the dead time of the DAQ system caused by the run switch done manually every $\sim 8$ hours, and the initialization failure of SciBar electronics which sometimes happened right after the run switch. In addition, there were occasionally detector down time due to maintenance works. Approximately $2 \%$ of beam spills were lost during the detector maintenance. Pedestals, the supplied high-voltages, and responses to cosmic-ray muons of each subdetector, and the response of SciBar to the LED light, are monitored continuously, and only the periods where all the sub-detectors are functioning are selected. Less than $1 \%$ of protons on target are rejected because of these cuts. 


\subsubsection{Summary of Data-taking}

In total, $2.64 \times 10^{20}$ protons on target were delivered to the beryllium target during the SciBooNE data. After all beam and detector quality cuts, $2.52 \times 10^{20}$ protons on target (POT) are usable for physics analyses. The data collection efficiency is $95.5 \%$. The number of POT for each run period is summarized in Table 3.4.

In these analyses, the full neutrino data sample is used, corresponding to $0.99 \times 10^{20}$ POT in RUN2 satisfying all data quality cuts, collected between October 2007 and April 2008.

Table 3.4: Summary of SciBooNE data-taking. The table shows the number of POT collected after the data quality cuts, as described in the text.

\begin{tabular}{lll}
\hline \hline Run & Period & POT \\
\hline Run 1 (Antineutrino) & Jun. 2007 - Aug. 2007 & $0.52 \times 10^{20}$ \\
Run 2 (Neutrino) & Oct. 2007 - Apr. 2008 & $0.99 \times 10^{20}$ \\
Run 3 (Antineutrino) & Apr. 2008 - Aug. 2008 & $1.01 \times 10^{20}$ \\
\hline \hline
\end{tabular}




\section{Chapter 4}

\section{Monte Carlo Simulation}

In this chapter, we describe the Monte Carlo (MC) simulations for the neutrino beam production, neutrino interactions with nuclei and the particle transportation in the detector.

\subsection{Neutrino Beam Simulation}

In order to predict the neutrino beam flux at the SciBooNE detector location, a Monte Carlo (MC) simulation, developed by the MiniBooNE Collaboration [57], is used. The simulation uses the GEANT4 framework [58].

\subsubsection{Simulation of Meson Productions}

The geometry and materials in the BNB target hall and decay region is implemented in the simulation code. Primary protons are generated according to the expected beam optics properties upstream of the target. The interactions of primary protons with the beryllium target are simulated based on the hadron interaction data. For the $\pi^{+}$production in proton-beryllium interactions, which is most important for these analyses, we uses experimental input from the HARP [59] and BNL E910 [60] experiments. We describe shortly the hadron production via the interactions of primary protons with the beryllium target in the simulation code. The detail description is in [56]. For $\pi^{+}, \pi^{-}$, and $K^{0}$ productions, the Sanford-Wang parametrization [61] is used to calculate the double differential cross section of a given meson species:

$$
\frac{d^{2} \sigma}{d p d \Omega}=C_{1} p^{C_{2}}\left(1-\frac{p}{p_{B}-C_{9}}\right) \exp \left(-C_{3} \frac{p^{C_{4}}}{p_{B}^{C_{5}}}-C_{6} \theta\left(p-C_{7} p_{B} \cos ^{C_{8}} \theta\right)\right)
$$

where $p$ is the total momentum of the meson, $\theta$ is the angle of the meson with respect to the incident proton, $p_{B}$ is the momentum of the incident proton, and $C_{1}, \ldots, C_{9}$ are parameters determined by fitting to the experimental data.

For $K^{+}$production, since no measurement exists at the BNB primary proton beam energy, the Feynman scaling hypothesis is employed to relate $K^{+}$production measurements at different proton beam energies to the expected production at the BNB proton beam energy. In this hypothesis, the Feynman scaling variable $x_{F} \equiv p_{\|}^{\mathrm{cm}} / p_{\|}^{\max , \mathrm{cm}}$ is used, 
where $p_{\|}^{\mathrm{cm}}$ and $p_{\|}^{\mathrm{max}, \mathrm{cm}}$ is the parallel component of the momentum of the produced particle in the center-of-mass frame and the maximum value of $p_{\|}^{\mathrm{cm}}$ for the given reaction, respectively. The double differential cross section using $x_{F}$ is expressed as:

$$
\frac{d^{2} \sigma}{d p d \Omega}=\frac{p^{2}}{E^{2}} C_{1}\left(1-\left|x_{F}\right|\right) \exp \left(-C_{2} p_{T}-C_{3}\left|x_{F}\right|^{C_{4}}-C_{5} p_{T}^{2}-C_{7}\left|p_{T} \times x_{F}\right|^{C_{6}}\right)
$$

where $p, p_{T}$ and $E$ are the momentum, the transverse component of the momentum and energy of $K^{+}$, respectively. For $K^{-}$production, the MARS hadronic interaction package [62] is used to determine the absolute double differential cross section.

\subsubsection{Simulation of Meson Decays}

The FORTRAN-based Monte Carlo code uses the output of the meson productions simulation as input, and generates the neutrino kinematics distributions from meson and muon decays. Current best knowledge of meson and muon decay branching fractions, and decay form factors in three-body semi-leptonic decays are used [56]. Polarization effects in muon decays are also accounted for.

\subsubsection{Neutrino Beam Flux Prediction at SciBooNE}

Once produced by the simulation, neutrinos are extrapolated along straight lines toward the SciBooNE detector. All neutrinos whose ray traces cross any part of the detector volume are considered for the SciBooNE flux predictions. No information from the SciBooNE and MiniBooNE neutrino flux measurements is used as the experimental input for the neutrino beam simulation.

Figure 4.1 shows the neutrino flux predictions at the SciBooNE detector location and as a function of neutrino energy. The spectra are averaged within $2.12 \mathrm{~m}$ from the beam center.* In the neutrino mode running (positive horn polarity), a expected total neutrino flux per POT is $2.2 \times 10^{-8} \mathrm{~cm}^{-2}$ at the SciBooNE detector location, with a mean neutrino energy of $0.7 \mathrm{GeV}$. The flux is dominated by muon neutrinos (92.92\% of total), with small contributions from muon antineutrinos (6.48\%), electron neutrinos $(0.54 \%)$ and electron antineutrinos (0.05\%).

On the other hand, in the antineutrino mode running (negative horn polarity), the expected total neutrino flux per proton on target is $1.3 \times 10^{-8} \mathrm{~cm}^{-2}$, and the mean neutrino energy is $0.6 \mathrm{GeV}$. The flux is dominated by muon antineutrinos $(83.85 \%$ of total), with contributions from muon neutrinos (15.58\%), electron neutrinos $(0.15 \%)$ and electron antineutrinos $(0.42 \%)$.

\subsubsection{Systematic Uncertainties in the Neutrino Flux Prediction}

The systematic uncertainties in the neutrino flux prediction come from several sources; proton delivery/optics, secondary particle productions, hadronic interactions in the target or horn and horn magnetic field. Detailed descriptions of each uncertainty are found elsewhere [57]. The systematic uncertainty in the total number of interactions at the

*The area used in this calculation covers the cross-sectional area of the SciBar detector which is $3 \mathrm{~m} \times 3 \mathrm{~m}$. 

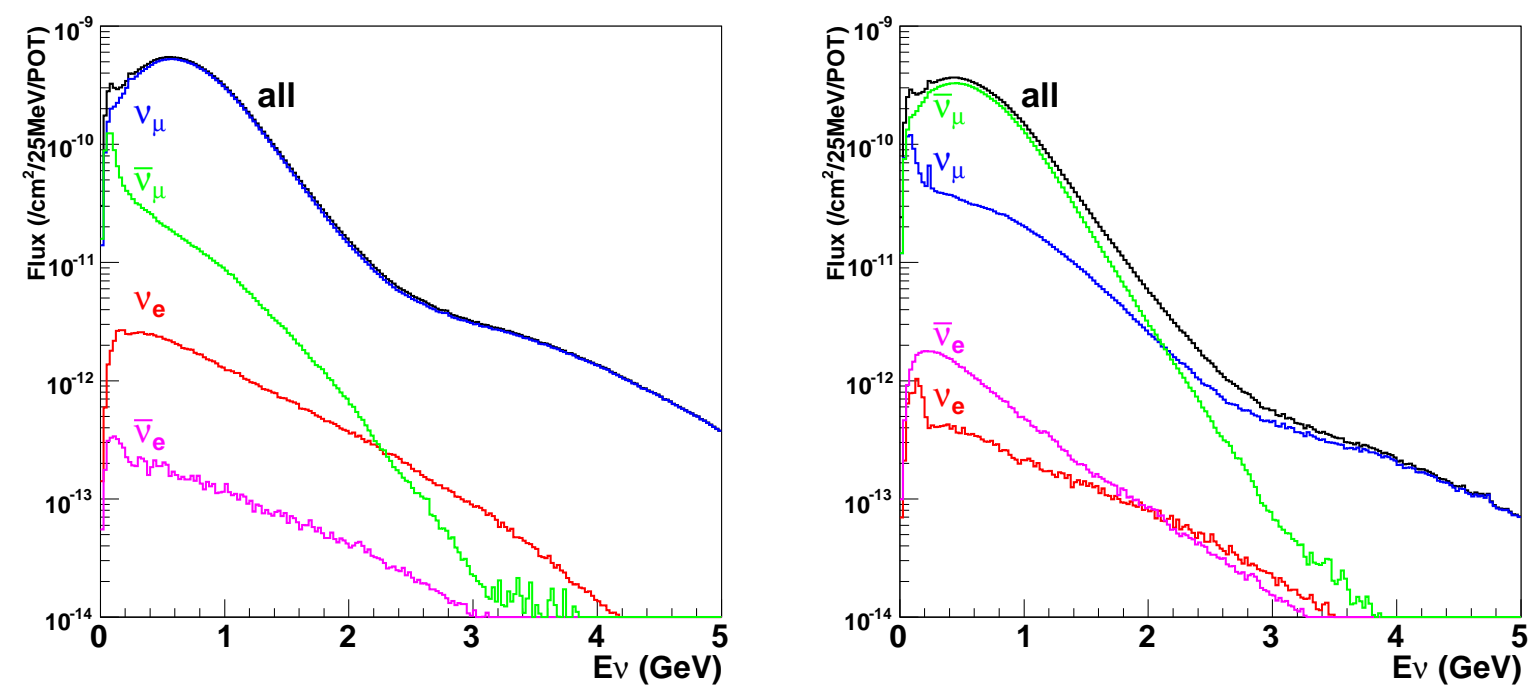

Figure 4.1: Neutrino flux predictions at the SciBooNE detector as a function of neutrino energy $E_{\nu}$, normalized per unit area, proton on target (POT) and neutrino energy bin width, in the neutrino mode (left) and the antineutrino mode running. The spectra are averaged within $2.12 \mathrm{~m}$ from the beam center. The total flux and contributions from individual neutrino flavors are shown.

SciBooNE detector is estimated to be $15 \%$ when only the neutrino flux uncertainties are taken into account. The error is dominated by the uncertainty of the $\pi^{+}$production cross section, which is $14 \%$. Because of the large uncertainty in the neutrino flux prediction, we measure cross section ratios in this thesis in order to reduce the systematic uncertainty on the cross section measurement, instead of measuring the absolute cross sections.

\subsection{Neutrino Interaction Simulation (NEUT)}

The neutrino interactions with nuclear targets are simulated with the NEUT program library $[63,64]$ which is used in the Kamiokande, Super-Kamiokande, K2K, and T2K experiments. NEUT generates neutrino interaction with nuclear targets such as protons, oxygen, carbon, and iron in the neutrino energy range from $100 \mathrm{MeV}$ to $100 \mathrm{TeV}$. Not only the primary neutrino interactions in nuclei but also secondary interactions of the mesons and hadrons with the nuclear medium are simulated.

\subsubsection{Neutrino-Nucleus Interactions}

In NEUT, the following neutrino interactions in both charged current (CC) and neutral current $(\mathrm{NC})$ are simulated:

- quasi-elastic scattering $\left(\nu N \rightarrow \ell N^{\prime}\right)$

- single meson production $\left(\nu N \rightarrow \ell N^{\prime} m\right)$

- single gamma production $\left(\nu N \rightarrow \ell N^{\prime} \gamma\right)$ 
- coherent $\pi$ production $\left(\nu^{12} \mathrm{C}\left(\right.\right.$ or $\left.\left.^{56} \mathrm{Fe}\right) \rightarrow \ell \pi{ }^{12} \mathrm{C}\left(\mathrm{or}^{56} \mathrm{Fe}\right)\right)$

- deep inelastic scattering $\left(\nu N \rightarrow \ell N^{\prime}\right.$ hadrons $)$

where $N$ and $N^{\prime}$ are the nucleons (proton or neutron), $\ell$ is the lepton, and $m$ is the meson. Figure 4.2 shows neutrino-nucleus cross sections per nucleon divided by neutrino energy. The expected number of $\nu_{\mu}$ interactions in the SciBar fiducial volume at SciBooNE is summarized in Table 4.1.

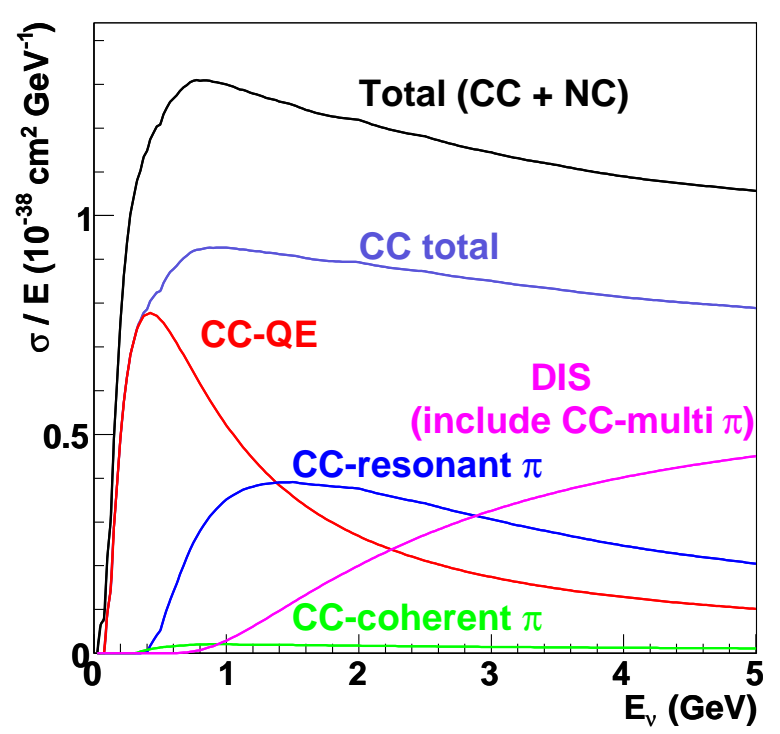

Figure 4.2: Neutrino-nucleus cross sections per nucleon divided by neutrino energy.

Table 4.1: The expected number and fraction of events in each neutrino interaction estimated by NEUT at the SciBooNE detector location with the neutrino beam exposure of $0.99 \times 10^{20}$ protons on target. The 10.6 ton fiducial volume of the SciBar detector is assumed. The $\mathrm{CC}$ and $\mathrm{NC}$ interactions are abbreviated as $\mathrm{CC}$ and $\mathrm{NC}$, respectively.

\begin{tabular}{lrr}
\hline \hline Interaction Type & \# Events & Fraction(\%) \\
\hline CC quasi-elastic & 53,363 & 41.4 \\
CC single $\pi$ via resonances & 29,688 & 23.1 \\
CC coherent $\pi$ & 1,771 & 1.4 \\
CC single meson except $\pi$ & 839 & 0.7 \\
CC DIS & 6,074 & 4.7 \\
NC elastic & 22,521 & 17.5 \\
NC single $\pi^{0}$ via resonances & 6,939 & 5.4 \\
NC coherent $\pi^{0}$ & 1,109 & 0.9 \\
NC single meson except $\pi^{0}$ & 4,716 & 3.7 \\
NC DIS & 1,768 & 1.4 \\
\hline \hline
\end{tabular}




\section{Quasi-elastic Scattering}

The dominant interaction in the SciBooNE neutrino energy range is quasi-elastic scattering. For the quasi-elastic scattering, the formula by Llewellyn-Smith [65] is used in NEUT. The amplitude of this process is described by the product of the leptonic and hadronic weak currents. The hadronic current is expressed as

$$
<N^{\prime}\left|J_{\mu}^{\mathrm{had}}\right| N>=\cos \theta_{c} \bar{u}\left(N^{\prime}\right)\left[\gamma_{\mu} F_{V}^{1}\left(Q^{2}\right)+\frac{i \sigma_{\mu \nu} q^{\nu} \xi F_{V}^{2}\left(Q^{2}\right)}{2 m_{N}}+\gamma_{\mu} \gamma_{5} F_{A}\left(Q^{2}\right)\right] u(N),
$$

where $\theta_{c}$ is the Cabbibo angle, $\xi \equiv \mu_{p}-\mu_{n}=3.71$ is the difference of anomalous dipole moments between a proton and a neutron, and $m_{N}$ is the nucleon mass. The vector form factors, $F_{V}^{1}$ and $F_{V}^{2}$, are represented as

$$
\begin{aligned}
F_{V}^{1}\left(Q^{2}\right) & =\left(1+\frac{Q^{2}}{4 m_{N}^{2}}\right)^{-1}\left[G_{E}^{V}\left(Q^{2}\right)+\frac{Q^{2}}{4 m_{N}^{2}} G_{M}^{V}\left(Q^{2}\right)\right], \\
\xi F_{V}^{2}\left(Q^{2}\right) & =\left(1+\frac{Q^{2}}{4 m_{N}^{2}}\right)^{-1}\left[G_{M}^{V}\left(Q^{2}\right)-G_{E}^{V}\left(Q^{2}\right)\right],
\end{aligned}
$$

where $G_{E}^{V}$ and $G_{M}^{V}$ are the electric and magnetic Sachs form factors, given by

$$
G_{E}^{V}\left(Q^{2}\right)=\frac{1}{\left(1+\frac{Q^{2}}{M_{V}^{Q E^{2}}}\right)^{2}} \quad, \quad G_{M}^{V}\left(Q^{2}\right)=\frac{1+\xi}{\left(1+\frac{Q^{2}}{M_{V}^{Q E^{2}}}\right)^{2}}
$$

The axial-vector form factor, $F_{A}$, is given by

$$
F_{A}\left(Q^{2}\right)=\frac{g_{A}}{\left(1+\frac{Q^{2}}{M_{A}^{Q E^{2}}}\right)^{2}}
$$

where $g_{A}=-1.23$ is determined from neutron decay measurements. Both the vector and axial-vector form factors are assumed to be dipole. The vector mass $\left(M_{V}^{Q E}\right)$ and the axial vector mass $\left(M_{A}^{Q E}\right)$ for the quasi elastic scattering are set to be $0.84 \mathrm{GeV} / c^{2}$ and $1.21 \mathrm{GeV} / c^{2}$, respectively, as suggested by recent results $[66,67]$.

The differential cross section is expressed as

$$
\frac{d \sigma}{d Q^{2}}=\frac{m_{N}^{2} G_{F}^{2} \cos ^{2} \theta_{c}}{8 \pi E_{\nu}^{2}}\left[A\left(Q^{2}\right) \mp B\left(Q^{2}\right) \frac{s-u}{m_{N}^{2}}+C\left(Q^{2}\right) \frac{(s-u)^{2}}{m_{N}^{4}}\right],
$$

where $E_{\nu}$ is the incident neutrino energy, $s-u \equiv 4 m_{N} E_{\nu}-Q^{2}-m_{\ell}^{2}, m_{\ell}$ is the mass of the outgoing lepton, and

$$
\begin{aligned}
A\left(Q^{2}\right)= & \frac{m_{\ell}^{2}+Q^{2}}{4 m_{N}^{2}}\left[\left(4+\frac{Q^{2}}{m_{N}^{2}}\right)\left|F_{A}\right|^{2}-\left(4-\frac{Q^{2}}{m_{N}^{2}}\right)\left|F_{V}^{1}\right|^{2}\right. \\
& +\frac{Q^{2}}{m_{N}^{2}}\left|\xi F_{V}^{2}\right|^{2}\left(1-\frac{Q^{2}}{4 m_{N}^{2}}\right)+\frac{4 Q^{2} F_{V}^{1} \xi F_{V}^{2}}{m_{N}^{2}} \\
& \left.-\frac{m_{\ell}^{2}}{m_{N}^{2}}\left(\left|F_{V}^{1}+\xi F_{V}^{2}\right|^{2}+\left|F_{A}\right|^{2}\right)\right] \\
B\left(Q^{2}\right)= & -\frac{Q^{2}}{m_{N}^{2}} F_{A}\left(F_{V}^{1}+\xi F_{V}^{2}\right) \\
C\left(Q^{2}\right)= & \frac{1}{4}\left(\left|F_{A}\right|^{2}+\left|F_{V}^{1}\right|^{2}+\frac{Q^{2}}{m_{N}^{2}}\left|\frac{\xi F_{V}^{2}}{2}\right|^{2}\right)
\end{aligned}
$$


To obtain the cross sections for neutral current elastic scattering, we use the following relations $[68,69]$ :

$$
\begin{aligned}
\sigma(\nu p \rightarrow \nu p) & =0.153 \times \sigma\left(\nu n \rightarrow \mu^{-} p\right) \\
\sigma(\bar{\nu} p \rightarrow \bar{\nu} p) & =0.218 \times \sigma\left(\bar{\nu} p \rightarrow \mu^{+} n\right) \\
\sigma(\nu n \rightarrow \nu n) & =1.5 \times \sigma(\nu p \rightarrow \nu p) \\
\sigma(\bar{\nu} n \rightarrow \bar{\nu} n) & =1.0 \times \sigma(\nu n \rightarrow \nu n)
\end{aligned}
$$

For scattering off nucleons in the nucleus, we use the relativistic Fermi gas model of Smith and Moniz [70]. The nucleons are treated as quasi-free particles and the Fermi motion of nucleons along with the Pauli exclusion principle is taken into account. The momentum distribution of the target nucleon is assumed to be flat up to a fixed Fermi surface momentum of $217 \mathrm{MeV} / c$ for carbon and $250 \mathrm{MeV} / c$ for iron. The same Fermi momentum distribution is also used for all of the other nuclear interactions. The nuclear potential is set to $27 \mathrm{MeV}$ for carbon and $32 \mathrm{MeV}$ for iron.

\section{Single Meson Production via Baryon Resonances}

The second most probable interaction in SciBooNE is the resonant single meson production of $\pi, K$, and $\eta$, described by the model of Rein and Sehgal [18]. The main signal in this analysis is NC single $\pi^{0}$ production via baryon resonances. The model assumes an intermediate baryon resonance, $N^{*}$ :

$$
\begin{aligned}
\nu N & \rightarrow \ell N^{*}, \\
N^{*} & \rightarrow N^{\prime} m \quad(m=\pi, \eta, K) .
\end{aligned}
$$

The double differential cross section of single meson production depends on the amplitude for the production of a given resonance and the probability of the baryon resonance decay to the meson:

$$
\frac{d^{2} \sigma}{d Q^{2} d \nu}=\frac{1}{32 \pi m_{N} E_{\nu}^{2}} \cdot \frac{1}{2} \sum_{\text {spins }}\left|T\left(\nu N \rightarrow \ell N^{*}\right)\right|^{2} \cdot \delta\left(W^{2}-M^{2}\right),
$$

where $W$ is the hadronic invariant mass and $T\left(\nu N \rightarrow \ell N^{*}\right)$ is given as

$$
\begin{aligned}
T\left(\nu N \rightarrow \ell N^{*}\right) & =-4 G_{F} \cos \theta_{c} M E\left[\sqrt{\frac{-q^{2}}{Q^{2}}}<N^{*}\left|u F_{-}-v F_{+}\right| N>\right. \\
& \left.+\frac{m_{N}}{M} \sqrt{2 u v}<N^{*}\left|F_{0}\right| N>\right] .
\end{aligned}
$$

Here, $u$ and $v$ are defined as

$$
u=\frac{E+E^{\prime}+Q}{2 E} \quad v=\frac{E+E^{\prime}-Q}{2 E}
$$

where the incident and final lepton energies are denoted by $E$ and $E^{\prime}$, respectively, and $Q$ is the modulus of the three momentum transfer in the Lab. We use FKR (FeynmanKislinger-Ravndal) baryon model [71] to express the baryon state $\left(|N>,| N^{*}\right\rangle$ in Eq. 4.18) and calculate $F_{ \pm}, F_{0}$ in Eq 4.18. 
In this model, for the baryon of three quarks, we have the $S U(6)$ spin and flavor multiplets $(\mathbf{5 6}, \mathbf{7 0}$ and 20) and spatial excitations of two independent three-dimensional modes of internal harmonic oscillation among the three particles. Therefore, baryon state is generally expressed as

$$
\sum_{i} \alpha_{i}\left|f_{1}, f_{2}, f_{3}>_{i}\right| s_{1}, s_{2}, s_{3}>_{i} \mid n(\text { space })>_{i}
$$

where, $f_{j}$ and $s_{j}$ represent the flavor and spin of the $j$-th quark, respectively, and $\mid n($ space $)>$ is the spatial wave function at the $n$-th excitation. The way to obtain the explicit form of Eq. 4.20 for each baryon is described in the Appendix of [71].

To calculate $F_{ \pm}, F_{0}$ in Eq 4.18, the four-dimensional harmonic oscillator Hamiltonian is used. We just put results here without the details of calculations. For the charged current, they obtained

$$
\begin{aligned}
& F_{ \pm}^{C C}=-9 \tau^{+}\left[\left(T^{V} \pm T^{A}\right) a_{\mp}+\left(R^{V} \pm R^{A}\right) \sigma_{ \pm}\right] e^{-\lambda a_{z}} \\
& F_{0}^{C C}=9 \tau^{+}\left[S+C \sigma_{z}+B \vec{\sigma} \cdot \vec{a}\right] e^{-\lambda a_{z}}
\end{aligned}
$$

For the neutral current, they obtained

$$
\begin{aligned}
F_{ \pm}^{N C} & =-9 \frac{\tau^{3}}{2}\left[\left(T^{V} \pm T^{A}\right) a_{\mp}+\left(R^{V} \pm R^{A}\right) \sigma_{ \pm}\right] e^{-\lambda a_{z}} \\
& -9 \sin ^{2} \theta_{w}\left(\tau^{3}+1\right)\left(T^{V} a_{\mp}+R^{V} \sigma_{ \pm}\right) e^{-\lambda a_{z}} \\
F_{0}^{N C} & =9 \frac{\tau^{3}}{2}\left[S+C \sigma_{z}+B \vec{\sigma} \cdot \vec{a}\right] e^{-\lambda a_{z}} \\
& -9 \sin ^{2} \theta_{w}\left(\tau^{3}+1\right) S e^{-\lambda a_{z}} .
\end{aligned}
$$

Here the isospin operator $\left(t^{+}, t^{3}\right)$, spin operator $(1, \vec{\sigma})$ and oscillator operators $(\vec{a})$ act on the flavor, spin and space sectors in the baryon wave function (Eq. 4.20), respectively, and other parameters are defined as

$$
\begin{aligned}
\lambda & =\sqrt{\frac{2}{\Omega}} \frac{m_{N}}{W} Q \\
T^{V} & =\frac{1}{3 W} \sqrt{\frac{2}{\Omega}} G^{V}\left(q^{2}\right) \\
R^{V} & =\sqrt{2} \frac{m_{N}}{W} \frac{\left(W+m_{N}\right) Q}{\left(W+m_{N}\right)^{2}-q^{2}} G^{V}\left(q^{2}\right), \\
S & =-\frac{q^{2}}{Q^{2}} \frac{3 m_{N}+q^{2}-m_{N}^{2}}{6 m_{N}^{2}} G^{V}\left(q^{2}\right), \\
T^{A} & =\frac{1}{2} \sqrt{\frac{2}{\Omega}} \frac{m_{N}}{W} \frac{Q}{\left(W+m_{N}\right)^{2}-q^{2}} G^{A}\left(q^{2}\right), \\
R^{A} & =\frac{\sqrt{2}}{8 W}\left(W+m_{N}+\frac{2 n W^{2}}{\left(W+m_{N}\right)^{2}-q^{2}}\right) G^{A}\left(q^{2}\right), \\
B & =\frac{1}{4 W} \sqrt{\frac{2}{\Omega}}\left(1+\frac{W^{2}-m_{N}^{2}+q^{2}}{\left(W+m_{N}\right)^{2}-q^{2}}\right) G^{A}\left(q^{2}\right), \\
C & =\frac{1}{8 m_{N} Q}\left(W^{2}-m_{N}^{2}+n \Omega \frac{W^{2}-m_{N}^{2}+q^{2}}{\left(W+m_{N}\right)^{2}-q^{2}}\right) G^{A}\left(q^{2}\right) .
\end{aligned}
$$


The transition form factors $G^{V, A}\left(q^{2}\right)$ are expressed as

$$
G^{V, A}\left(q^{2}\right)=\left(1-\frac{q^{2}}{4 m_{N}^{2}}\right)^{1 / 2-n}\left(\frac{1}{1-q^{2} / M_{V, A}^{1 \pi}{ }^{2}}\right)^{2}
$$

where $n$ is the number of excitations of baryon spatial wave function (Eq. 4.20). Both the vector and axial-vector form factors are assumed to be dipole. The vector mass $\left(M_{V}^{1 \pi}\right)$ and axial vector mass $\left(M_{A}^{1 \pi}\right)$ for the single meson production, is set to be $0.84 \mathrm{GeV} / c^{2}$ and $1.21 \mathrm{GeV} / c^{2}$, respectively.

For resonances with a finite decay width, the double differential cross sections can be derived by replacing the $\delta$-function with a Breit-Wigner formula:

$$
\delta\left(W^{2}-M^{2}\right) \rightarrow \frac{1}{2 \pi} \frac{\Gamma}{(W-M)^{2}+\Gamma^{2} / 4} .
$$

All intermediate baryon resonances with mass less than $2 \mathrm{GeV} / c^{2}$ are included. Those baryon resonances with mass greater than $2 \mathrm{GeV} / c^{2}$ are simulated as deep inelastic scattering. Lepton mass effects from the non-conservation of lepton current and the pion-pole term in the hadronic axial vector current are included in the simulation $[72,73]$.

To determine the angular distribution of a pion in the final state, Rein's method [74] is used for the $P_{33}(1232)$ resonance. For other resonances, the directional distribution of the generated pion is set to be isotropic in the resonance rest frame. The angular distribution of $\pi^{+}$has been measured for $\nu_{\mu} p \rightarrow \mu^{-} p \pi^{+}[75]$ and the results agree well with the prediction of NEUT. Pauli blocking is accounted for in the decay of the baryon resonance by requiring the momentum of the nucleon to be larger than the Fermi surface momentum. Pion-less $\Delta$ decay is also taken into account, where $20 \%$ of the events do not have a pion and only the lepton and nucleon are generated [76].

\section{Coherent Pion Production}

The model of Rein and Sehgal $[32,35]$ is used to simulate coherent pion production in NEUT. Based on Adler's PCAC theorem, the differential cross section for $Q^{2}=0$ is expressed as

$$
\left.\frac{d^{3} \sigma(\nu A \rightarrow \nu A \pi)}{d x d y d t}\right|_{Q^{2}=0}=\left.\frac{G_{F}^{2}}{\pi^{2}} f_{\pi}^{2} m_{N} E_{\nu}(1-y) \frac{\sigma(\pi A \rightarrow \pi A)}{d t}\right|_{E_{\nu} y=E_{\pi}},
$$

where $x=Q^{2} / 2 m_{N} \nu$ and $y=\nu / E_{\nu}$ are the Bjorken kinematic variables, $\nu$ is the energy transfer, $t$ is the square of the four-momentum transferred to the nucleus, $G_{F}$ is the weak coupling constant, $f_{\pi}$ is the pion decay constant, and $m_{N}$ is the mass of nucleon. The relation expresses the forward neutrino cross section at the nucleus $A$ in terms of the cross section for the process $\pi A \rightarrow \pi A$.

The extrapolation of the cross section to $Q^{2} \neq 0$ is done based on the method of hadron dominance, which is an extension of the model used in electromagnetic interactions, the vector meson dominance (VMD) model. The VMD model treats the electromagnetic current as a superposition of the contribution from the lightest vector mesons. Following the calculation of the VMD model, the cross section is then obtained by attaching a propagator term:

$$
\frac{d^{3} \sigma(\nu A \rightarrow \nu A \pi)}{d x d y d t}=\left.\frac{G_{F}^{2}}{\pi^{2}} f_{\pi}^{2} m_{N} E_{\nu}(1-y)\left(\frac{m_{A}^{2}}{Q^{2}+m_{A}^{2}}\right)^{2} \frac{\sigma(\pi A \rightarrow \pi A)}{d t}\right|_{E_{\nu} y=E_{\pi}}
$$


where $m_{A}$ is the axial vector mass, introduced practically in the propagator.

The pion-nucleus differential cross section is expressed as

$$
\frac{d \sigma(\pi A \rightarrow \pi A)}{d t}=\left.\mathcal{A}^{2}\left|F_{A}(t)\right|^{2} \frac{d \sigma(\pi N \rightarrow \pi N)}{d t}\right|_{t=0}
$$

where $\mathcal{A}$ is the atomic number of the nucleus, $F_{A}(t)$ is the nuclear form factor (including the effect of pion absorption). With the aid of the optical theorem, the pion-nucleon differential cross section in the forward direction is given by

$$
\left.\frac{d \sigma(\pi N \rightarrow \pi N)}{d t}\right|_{t=0}=\frac{1}{16 \pi}\left[\sigma_{\text {tot }}^{\pi N}\right]^{2}\left(1+r^{2}\right), \quad r=\frac{\operatorname{Re} f_{\pi N}(0)}{\operatorname{Im} f_{\pi N}(0)}
$$

An average cross section from measurements of pion-deuteron scattering is used to obtain $\sigma_{\text {tot }}^{\pi N}$ in this model. For the nuclear form factor,

$$
\left|F_{A}(t)\right|^{2}=e^{-b|t|} F_{\mathrm{abs}}
$$

is used, where $b$ is related to the nuclear radius $R$, given by

$$
b=\frac{1}{3} R^{2} \quad\left(R=R_{0} \mathcal{A}^{1 / 3}\right) .
$$

The $F_{\text {abs }}$ is the attenuation factor representing the effect of pion absorption in the nucleus, expressed as

$$
F_{\text {abs }}=e^{-\langle x\rangle / \lambda},
$$

where $\langle x\rangle$ and $\lambda$ are the average path length traversed by the pion produced in the nucleus, and the absorption length, respectively. By assuming the nucleus is a homogeneous sphere with an uniform density, $\langle x\rangle$ and $\lambda$ are calculated as

$$
\langle x\rangle=\frac{3}{4} R, \quad \lambda=\mathcal{A}\left(\frac{4 \pi R^{3}}{3}\right)^{-1} \sigma_{\text {inel }}^{\pi N},
$$

where $\sigma_{\text {inel }}^{\pi N}$ is the pion-nucleon inelastic cross section. As a result of this,

$$
F_{\text {abs }}=\exp \left(-\frac{9 \mathcal{A}^{1 / 3}}{16 \pi R_{0}^{2}} \sigma_{\text {inel }}^{\pi N}\right)
$$

is obtained. Hence, the differential cross section for coherent pion production in the model of Rein and Sehgal is expressed as

$$
\frac{d^{3} \sigma(\nu A \rightarrow \nu A \pi)}{d x d y d t}=\frac{G_{F}^{2}}{\pi^{2}} f_{\pi}^{2} m_{N} E_{\nu}(1-y)\left(\frac{m_{A}^{2}}{Q^{2}+m_{A}^{2}}\right)^{2} \frac{\mathcal{A}^{2}}{16 \pi}\left[\sigma_{\text {tot }}^{\pi N}\right]^{2}\left(1+r^{2}\right) e^{-b|t|} F_{\text {abs }}
$$

The Rein and Sehgal model predicts three features of coherent pion production as follows

1. Since the axial parts of the neutral and charged currents form a triplet in isospace, we are led to $f_{\pi^{0}}^{2}=\left(\sqrt{1 / 2} f_{\pi^{+}}\right)^{2}=(1 / 2) f_{\pi^{+}}^{2}=(1 / 2)\left(0.93 m_{\pi}\right)^{2}$. Therefore, the model predicts the relation between the neutral and charged current coherent pion production cross sections as $\sigma(\mathrm{CC})=2 \times \sigma(\mathrm{NC})$. The relation is slightly modified since the charged current cross section is modified by the lepton mass correction [35]. 
2. The $A$ dependence of the cross section turns out to be approximately $\mathcal{A}^{1 / 3}$. The behavior results roughly from a product $\mathcal{A}^{4 / 3} b^{-1} b^{-1 / 2}$, where $b \sim R^{2} \sim \mathcal{A}^{2 / 3}$. The first factor is the effect of nuclear coherence, including the effects of pion absorption, the second factor comes from integration of $e^{-b|t|}$ over $t$, the third one comes from integration over $x$.

3. The model predicts the same cross section for coherent pion production by neutrinos and antineutrinos.

In our MC simulation, we set the axial vector mass, $m_{A}$, to $1.0 \mathrm{GeV} / c^{2}$, and the nuclear radius parameter, $R_{0}$, is set to $1.0 \mathrm{fm}$. For the total and inelastic pion-nucleon cross sections, $\sigma_{\text {tot }}^{\pi N}$ and $\sigma_{\text {inel }}^{\pi N}$, respectively, the fitted results given in Rein and Sehgal's paper are used.

\section{Deep Inelastic Scattering (DIS)}

The double differential cross section for charged current deep inelastic scattering (DIS) is calculated as:

$$
\begin{aligned}
\frac{d^{2} \sigma}{d x d y} & =\frac{G_{F}^{2} m_{N} E_{\nu}}{\pi}\left[\left(1-y+\frac{1}{2} y^{2}+C_{1}\right) F_{2}(x) \pm y\left(1-\frac{1}{2} y+C_{2}\right) x F_{3}(x)\right] \\
C_{1} & =\frac{m_{\ell}^{2}(y-2)}{4 m_{N} E_{\nu} x}-\frac{m_{N} x y}{2 E_{\nu}}-\frac{m_{\ell}^{2}}{4 E_{\nu}^{2}} \\
C_{2} & =-\frac{m_{\ell}^{2}}{4 m_{N} E_{\nu} x}
\end{aligned}
$$

where $x \equiv Q^{2} /\left(2 m_{N}\left(E_{\nu}-E_{\ell}\right)+m_{N}^{2}\right)$ and $y \equiv\left(E_{\nu}-E_{\ell}\right) / E_{\nu}$ are the Bjorken scaling parameters, and $E_{\ell}$ is the energy of the final state lepton. The nucleon structure functions, $F_{2}$ and $x F_{3}$, are calculated using the GRV98 parton distribution functions [77]. In addition, we have included the corrections in the small $Q^{2}$ region developed by Bodek and Yang [78]. In the calculation, the hadronic invariant mass, $W$, is required to be larger than $1.3 \mathrm{GeV} / c^{2}$.

The multi-hadron final states are simulated with two models. In the range of $1.3<$ $W<2.0 \mathrm{GeV} / c^{2}$, a custom-made program [79] is employed. For the events with $W>$ $2 \mathrm{GeV} / c^{2}$, PYTHIA/JETSET [80] is used to calculate the kinematics of hadronic final states.

To obtain the cross sections for neutral current deep inelastic scattering, we use the following relations:

$$
\begin{aligned}
& \frac{\sigma(\nu \text { NC-DIS })}{\sigma(\nu \text { CC-DIS })}= \begin{cases}0.26 & \left(E_{\nu} \leq 3 \mathrm{GeV}\right) \\
0.26+0.04 \times\left(E_{\nu} / 3-1\right) & \left(3<E_{\nu}<6 \mathrm{GeV}\right) \\
0.30 & \left(E_{\nu} \geq 6 \mathrm{GeV}\right)\end{cases} \\
& \frac{\sigma(\bar{\nu} \text { NC-DIS })}{\sigma(\bar{\nu} \text { CC-DIS })}= \begin{cases}0.39 & \left(E_{\nu} \leq 3 \mathrm{GeV}\right) \\
0.39-0.02 \times\left(E_{\nu} / 3-1\right) & \left(3<E_{\nu}<6 \mathrm{GeV}\right) \\
0.37 & \left(E_{\nu} \geq 6 \mathrm{GeV}\right)\end{cases}
\end{aligned}
$$

These relations are estimated from the experimental results $[81,82]$. 


\subsubsection{Intra-nuclear Interactions}

The interactions of mesons and nucleons produced via neutrino interactions with nuclear matters are simulated. These interactions are treated by using a cascade model, and each of the particles is traced until it escapes from the nucleus. The neutrino interaction position in the nucleus is calculated using the Wood-Saxon type nucleon density distribution:

$$
\rho(r)=\frac{Z}{A} \rho_{0}\left[1+\exp \left(\frac{r-c}{a}\right)\right]^{-1},
$$

where $\rho_{0}=0.48 m_{\pi}^{3}, A$ and $Z$ are the mass number and atomic number of the nucleus, respectively. For carbon nucleus, $a=0.52 \mathrm{fm}$ and $c=2.36 \mathrm{fm}$ [83]. Fermi motion of nucleons in the nucleus and Pauli blocking effect are taken into account in the simulation. The Fermi surface momentum at the interaction point is defined as

$$
p_{F}(r)=\left(\frac{3}{2} \pi^{2} \rho(r)\right)^{\frac{1}{3}} .
$$

\section{Pion Interactions}

The interactions of pions are most important for the analysis in this thesis. The inelastic scattering, charge exchange and absorption of pions in the nuclei are simulated. The interaction cross sections of pions in the nuclei are calculated using the model by Salcedo et al. [84], which agrees well with past experimental data [85]. If inelastic scattering or charge exchange occurs, the direction and momentum of pions are determined by using results from a phase shift analysis of pion-nucleus scattering experiments [86].

In SciBooNE, approximately $15 \%$ of pions produced via neutrino interaction are absorbed in carbon, about $20 \%$ of pions are inelastically scattered, and the probability of charge exchange is $5 \%$.

\section{Nucleon Re-scattering}

Re-interactions of the recoil protons and neutrons produced in neutrino interactions are also simulated. Nucleon-nucleon interactions modify the outgoing nucleon's momentum and direction. Both elastic scattering and pion production are considered. The differential cross sections were obtained from nucleon-nucleon scattering experiments [87]. For pion production, the isobaric nucleon model [88] is used.

In SciBooNE, approximately $35 \%$ of protons produced via charged current quasi-elastic scattering interact inside of the carbon nucleus.

\subsection{Detector Simulation}

We use the GEANT4 framework for the detector simulation. The detector simulation includes a detailed information of the detector geometry.

\subsubsection{Simulation of Detector Responses}

In the detector simulation of SciBar, true energy deposition in a scintillator strips simulated by GEANT is converted to the number of photoelectrons using a conversion 
factor which is measured for each channel with cosmic-ray muons. Scintillator quenching is simulated using Birk's law with a measured value of Birk's constant. [54] The measured light attenuation length (Table 3.1) of each fiber is used in the simulation. Crosstalk between nearby MA-PMT channels is simulated using measured values (Table 3.1). To simulate the digitization of the PMT signal, the number of photoelectrons is smeared by Poisson statistics and by the PMT resolution, and then converted to ADC counts. A logical OR of 32 MA-PMT channels is made for each TDC channel, and the time of each hit is converted to TDC counts. Multiple TDC hits in each channel are simulated.

In the EC detector simulation, true energy deposition in scintillating fibers in the detector is converted to the number of photoelectrons using a conversion factor which is measured for each channel with cosmic-ray muons. The attenuation of light in the fiber is simulated using the measured attenuation length value $(400 \mathrm{~cm})$. The number of photoelectrons is smeared by Poisson statistics and by the PMT resolution, and then converted to ADC counts.

For the detector simulation of the MRD, true energy deposition in each scintillator is converted to ADC counts using the conversion factor measured with cosmic muons. The attenuation of light in the scintillator are simulated. Gaps between scintillator counters in each plane, which cause inefficiency $(1 \%)$, are included in the simulation. The time of energy deposition is digitized and converted into TDC counts.

In addition to the main components such as SciBar, EC and MRD, the particle transportation in the detector structure frame made of iron and the dark box made of wood is also simulated.

\subsubsection{Simulation of Pion Interaction in Detector}

The Bertini cascade model within GEANT4 [89] is used to simulate the interactions of hadrons with detector materials. Among all the hadronic interactions, the interactions of pions are most important to the analysis. To check the validity of the simulation in our interested energy region $\left(T_{\pi}<500 \mathrm{MeV}\right)$, the simulated cross section of pioncarbon interaction is compared with external measurements [85,90-94]. As a result, a $10 \%$ difference of the total cross section between the MC simulation and data is seen for higher energy pion. The difference is accounted for when we evaluate systematic errors.

\subsubsection{Neutrino Interactions in the Surrounding Material}

In addition to neutrino interactions inside the detector, we also simulate interactions in the surrounding material (the walls of the detector hall and soil). The density of material is assumed to be $2.15 \mathrm{~g} / \mathrm{cm}^{3}$ for the calculation of the interaction rate. The concrete material with the density of $2.15 \mathrm{~g} / \mathrm{cm}^{3}$ is used as the material for propagation of product particles. We generate events in a volume of $\pm 5 \mathrm{~m}$ in $x, y$, and $z$ direction in the SciBooNE coordinates. The details are described in Appendix B. 


\section{Chapter 5}

\section{Event Selection for Neutral Current $\pi^{0}$ Production}

\subsection{Overview}

As shown in Table 4.1, the fraction of $\mathrm{NC} \pi^{0}$ production to all neutrino interactions is only about $6 \%$. Hence, we need make $\mathrm{NC} \pi^{0}$ enriched sample by applying the selections. In this chapter, we describe the event selections in detail.

Figure 5.1 shows the event display of typical $\mathrm{NC} \pi^{0}$ candidate in SciBooNE. We aim to reconstruct and select events where $\mathrm{NC} \pi^{0}$ interaction is occurred inside SciBar detector and both two gamma rays from $\pi^{0}$ are converted into $e^{+} e^{-}$pairs also in SciBar. The detail of the $\mathrm{NC} \pi^{0}$ signal definition is described in Section 5.2.

Before we describe the $\mathrm{NC} \pi^{0}$ event selections, we describe the particle reconstruction in Section 5.3. This is because the reconstructed objects such as hits, clusters and tracks are used for the event selections. As shown in this chapter, we keep gamma ray tracks by removing the proton and muon tracks because most of particles making tracks in SciBar are protons or muons except for gamma rays. In addition, identifying the muons is important to reject the CC background events. Furthermore, as shown in Figure 5.1, a single gamma ray in SciBooNE energy range (a few hundred $\mathrm{MeV}$ ) makes one main track and additional hits or short tracks around the main track in SciBar. Therefore, we perform the track-based reconstruction, which is commonly used for all SciBooNE analyses. Then, we identify protons and muons using the reconstructed tracks. The reconstructed tracks is also used for the reconstruction of gamma rays and $\pi^{0}$.

We also describe CC sample in Section 5.4. The CC sample is used for the beam flux normalization. Finally, we present the event selections for the $\mathrm{NC} \pi^{0}$ production in Section 5.5

\subsection{Signal Definition}

We define an $\mathrm{NC} \pi^{0}$ interaction as an $\mathrm{NC}$ neutrino interaction in which at least one $\pi^{0}$ is emitted in the final state from the target nucleus, $\nu_{\mu} C \rightarrow \nu_{\mu} \pi^{0} X$ where $X$ represents the nuclear remnant and any combination of nucleons and mesons. We include such $\mathrm{NC} \pi^{0}$ interactions occurring only in SciBar as our signals. Unless otherwise indicated, our " $\mathrm{NC} \pi^{0}$ " means a $\mathrm{NC} \pi^{0}$ interaction occurring in SciBar but neither in EC nor MRD. 


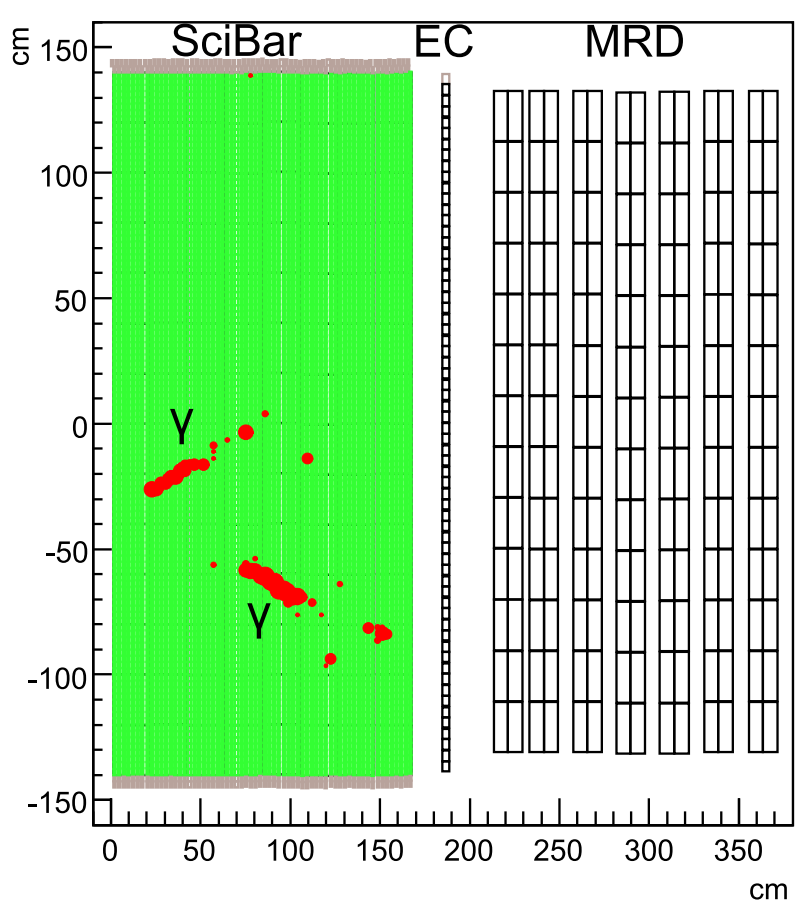

Figure 5.1: Event display of a typical $\mathrm{NC}^{0}$ event candidate in SciBooNE data. The neutrino beam runs from left to right in this figure, encountering SciBar, the EC and MRD, in that order. The circles on SciBar indicate ADC hits for which the area of the circle is proportional to the energy deposition in that channel. This event display shows the electromagnetic shower tracks from the pair conversions of the two $\pi^{0}$ decay photons.

According to our MC simulation, $96 \%$ of $\mathrm{NC} \pi^{0}$ events without any selection cuts have a single $\pi^{0}$ (85\% from a single $\pi^{0}$ without any other mesons and $11 \%$ from a single $\pi^{0}$ with charged mesons) and $4 \%$ have two $\pi^{0}$ s. Any $\pi^{0}$ emitted from the initial target nucleus constitutes a signal event whether it is created from the neutrino vertex or final state interactions. Events with a $\pi^{0}$ produced in the neutrino interaction but absorbed in the target nucleus are not included in the signal sample, nor are events in which $\pi^{0} \mathrm{~s}$ are produced by secondary particles interacting with the detector scintillator outside the target nucleus. We show the classification of the $\mathrm{NC}^{0}$ signal and non-signal in Figure 5.2.

We identify $\pi^{0}$ by two reconstructed gamma rays. Hence, both of the two gamma rays from $\pi^{0}$ must be converted into $e^{+} e^{-}$pairs in SciBar to be identified as a $\pi^{0}$ events. In fact, the number of signal events where both of the two gamma rays are converted in SciBar is only $30 \%$ of all signals. This means that the maximum $\mathrm{NC} \pi^{0}$ detection efficiency attainable is $30 \%$. We will describe the gamma conversion probability in detail in Section 6.6.2. 


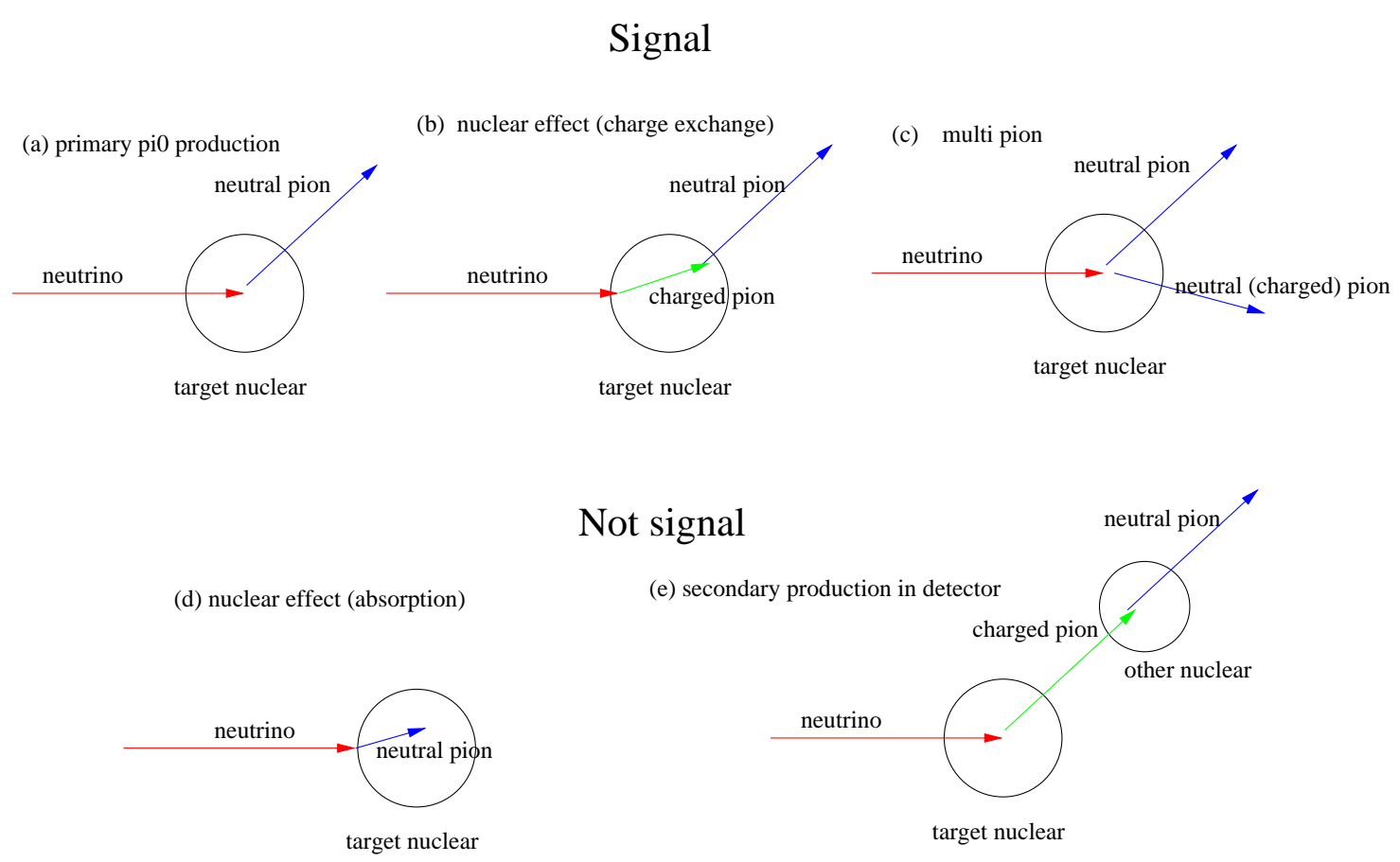

Figure 5.2: Classification of the NC $\pi^{0}$ signal and non-signal

\subsection{Particle Reconstruction}

\subsubsection{Overview}

Figure 5.3 shows a schematic view of the event reconstruction of SciBooNE. In SciBar, hits and 2D tracks, 3D tracks (we call just "tracks" hereafter) are obtained described as Section 5.3.2. In this analysis, 2D projections are used only for reconstructing tracks not directly used for the event selections or $\pi^{0}(\gamma)$ reconstruction. Based on the $d E / d x$ information, SciBar tracks are divided into proton-like tracks or non-proton-like tracks as described in Section 5.3.3. For the gamma ray reconstruction, extended tracks are obtained after the additional treatment to the non-proton-like tracks as described in Section 5.3.4.

The EC information are obtained as hits and clusters. We search the EC clusters matching with the tracks as described in Section 5.3.5. Such matched EC clusters are used for the muon identification and energy correction for gamma rays.

The MRD information are obtained as hits and tracks. We search the MRD hits or tracks matching with the SciBar tracks as described in Section 5.3.6. Such SciBar-MRD matched tracks are used for the muon identification.

\subsubsection{SciBar Track Reconstruction}

The first step of the SciBar event reconstruction is to search for two-dimensional tracks in each view of SciBar using a cellular automaton algorithm [95]. For tracking, the 


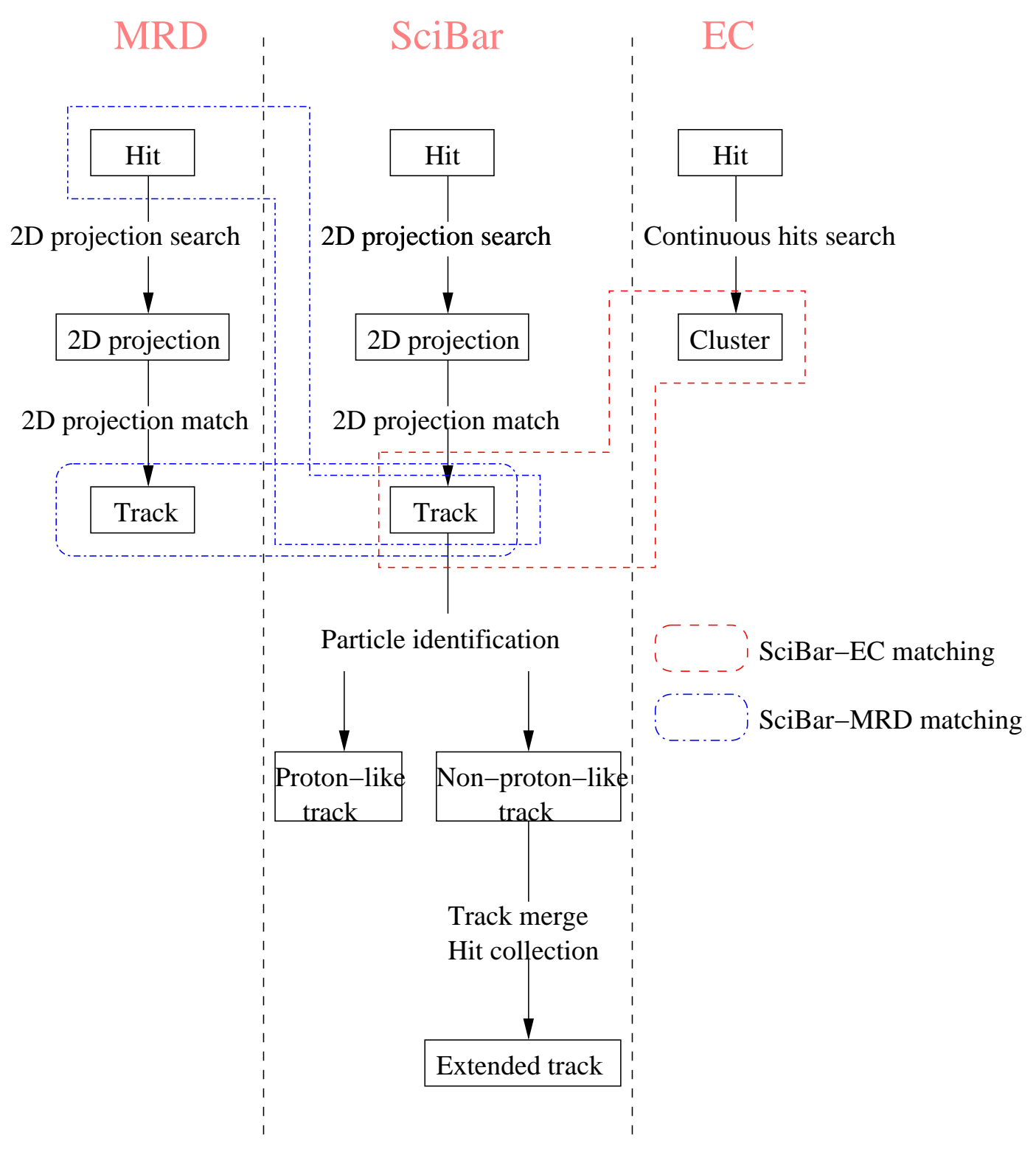

Figure 5.3: The schematic view of the event reconstruction of SciBooNE

hit threshold is set to 2.5 photo-electrons, corresponding to roughly $0.25 \mathrm{MeV}$. Three dimensional tracks are reconstructed by matching the timing and z-edges of the two dimensional projections. In order to match track projections in a three dimensional track, the timing difference between two two-dimensional projections is required to be less than $50 \mathrm{~ns}$, and the $z$-edge difference must be less than $6.6 \mathrm{~cm}$ for upstream and downstream edges.

*The previous SciBooNE result such as the measurement of charged current coherent production [56] used 2.0 photo-electrons threshold. We noticed that the data/MC discrepancies of the gamma ray reconstructions such as energy, direction and number of collected hits are large around 2.0 photo-electrons threshold. Hence, we decided to use 2.5 photo-electrons threshold. 


\subsubsection{Particle Identification Parameter}

The SciBar detector has the capability to distinguish protons from other particles by using $d E / d x$ since the energy deposit of recoil protons at SciBooNE energies is larger than minimum ionizing energy deposit. We define a muon confidence level (MuCL) using the observed energy deposit per layer for all reconstructed tracks [49]. The MuCL of the proton tracks tends to be close to 0 while the MuCL of other tracks tends to be close to 1 . Figure 5.4 shows the distribution of the MuCL. For the MC simulation, the contribution from gamma rays, muons, protons and other particles are separately shown, respectively. Tracks with MuCL less than 0.03 are called proton-like tracks, otherwise called non-proton-like tracks.

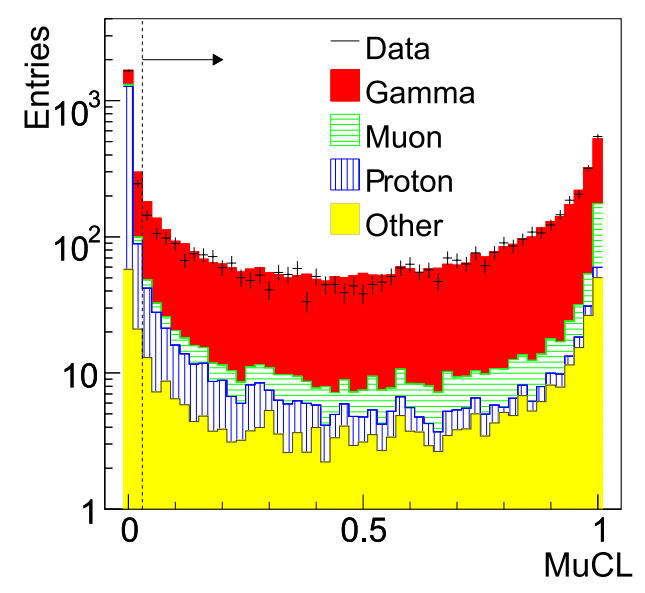

Figure 5.4: Distribution of MuCL of tracks

\subsubsection{Extended Track}

As mentioned at the beginning of this chapter, the typical gamma rays make one main track and additional short tracks or hits around the main track in SciBar. To reconstruct the energy of gamma rays, we need to collect such additional hits and short tracks. In case of additional short tracks, we need to relate the short tracks to the main track and handle them as a single gamma ray not two different particles. Hence, we introduce new reconstructed tracks called "extended tracks" based on the original reconstructed tracks.

Reconstructed tracks are extended in two ways to improve energy reconstruction of gamma rays within SciBar. The first step is merging two or more tracks if they are nearly co-linear, because electromagnetic showers can form separate hit clusters in SciBar resulting in two or more tracks. The second step is collecting hits around merged tracks. Electromagnetic showers sometimes deposit energy around the main track and these hits are missed by the track reconstruction algorithm. Hits within $20 \mathrm{~cm}$ from 2D projections of the merged track (i.e., after the first step) for each view but not assigned to any track are added to the extended track. The methods described above are applied only to non-proton-like tracks. For the energy reconstruction, we use charge information of hits associated with original tracks as well as newly assigned hits. For reconstructing the 
directions of gamma rays, we fit positions of hits in all original tracks in the extended track with a straight line and do not use hits newly collected in the second step. The details of the extended track is described in Appendix. A.

\subsubsection{SciBar-EC Matching}

Some particles can escape SciBar and deposit energy in the EC. After event reconstruction in SciBar we search for EC clusters aligned with tracks from SciBar. One EC cluster is defined as a collection of neighboring EC hits. For an EC hit, the pulse heights of both side PMTs are required to be above threshold, which is set to 3 times the width of each pedestal - about $7 \mathrm{MeV}$. The energy of an EC hit is the geometric average of the two PMTs. The center of an EC cluster is defined as the energy-weighted average of hits in the cluster. To match the EC cluster to the SciBar track, the EC cluster is required to be within $10 \mathrm{~cm}$ of the extrapolated two-dimensional projections of the SciBar track in each EC plane. The energy of matched EC clusters is added to the corresponding extended tracks. The details of the SciBar-EC matching is described in Appendix. A.

\subsubsection{SciBar-MRD Matching}

A track in SciBar matched to a track or hits in MRD is called the SciBar-MRD matched track. For matching the MRD track to a SciBar track, the upstream edge of the MRD track is required to be on either one of the first two layers of MRD. The transverse distance between the two tracks at the first layer of MRD must be less than $30 \mathrm{~cm}$. The requirement on the difference between track directions is given by $\left|\theta_{\mathrm{MRD}}-\theta_{\mathrm{SB}}\right|<\theta_{\max }$, where $\theta_{\max }$ is a function of the length of the MRD track in the range between 0.4 radian and 1.1 radians. If no MRD track is found, we extrapolate the SciBar track to the MRD and search for nearby contiguous hits in MRD. For matching MRD hits to the SciBar track, the MRD hit is required to be within a cone with an aperture of \pm 0.5 radian and a transverse offset within $10 \mathrm{~cm}$ of the extrapolated SciBar track at the upstream edge of MRD. The timing difference between the SciBar track and the track or hits in MRD is required to be within 100 nsec.

\subsection{Normalization (Charged-Current Event Sam- ple)}

To identify charged current events, we search for events with at least one SciBarMRD matched track. We reject events with hits associated with the muon track on the most upstream layer of SciBar to eliminate neutrino-induced incoming particles from the upstream wall or soil. The neutrino interaction vertex for CC events is reconstructed as the upstream edge of the muon track. We select events whose vertices are in the SciBar fiducial volume. The fiducial volume is defined as

$$
\left\{\begin{array}{l}
-130 \mathrm{~cm}<\mathrm{x}<130 \mathrm{~cm} \\
-130 \mathrm{~cm}<\mathrm{y}<130 \mathrm{~cm} \\
2.62 \mathrm{~cm}<\mathrm{z}<157.2 \mathrm{~cm}(2 \mathrm{nd}-60 \text { th layer })
\end{array}\right.
$$

The total mass in the fiducial volume is 10.6 tons. The time of the muon track is required to be within a $2 \mu \mathrm{s}$ window around the beam pulse. Finally, we require the muon track to 
stop in the MRD. The MRD-stopped event sample [49] serves as the normalization sample in the cross-section ratio measurement. Unless otherwise indicated, the MC distributions in this paper are normalized by using the MRD-stopped data sample.

\subsection{Event Selection for $\mathrm{NC} \pi^{0}$}

\subsubsection{Overview}

The clear feature of NC $\pi^{0}$ production is two gamma rays, coming from the decay of the $\pi^{0}$, converted into two $e^{+} e^{-}$pairs. Background events can be categorized into two types. One is called an internal background. Internal backgrounds are neutrino interactions other than $\mathrm{NC} \pi^{0}$ (mainly CC) within SciBar. The other is called an external background. External backgrounds come from neutrino interactions in the material outside of the detector volume (support structure, walls and soil-so called dirt background events) as well as from cosmic rays. In dirt background events, neutrinos interact with materials such as the walls of the experimental hall or soil and produce secondary particles which deposit energy within SciBar's fiducial volume. The contribution of accidental cosmic rays in the event samples is small and accurately estimated by data taken with off-beam timing; the fraction of accidental cosmic ray events is $1.8 \%$ after all event selection cuts. Data distributions shown hereafter are after subtracting the cosmic backgrounds. The event selection cuts for $\mathrm{NC} \pi^{0}$ production are developed to select events with two gamma rays while rejecting the above background events.

The event selection cuts are summarized as follows.

(1) Number of tracks

(2) Fiducial volume cut

(3) First layer veto

(4) Side escaping track rejection cut

(5) Decay electron rejection cut

(6) Track disconnection cut

(7) Electron catcher cut

(8) Number of extended tracks

(9) $\pi^{0}$ vertex cut

(10) $\pi^{0}$ mass cut

Here, we describe the outline of the event selection cuts. We describe the details of each cuts in following selections. First, at least two tracks starting in SciBar are selected at the selections $(1)(2)$. Charged particles from the outside of SciBar are rejected at the selection (3). Muons escaping from the side of SciBar or stopping in SciBar are rejected at the selections (4)(5). At the selection (6), we reject charged current events with two particles whose vertices are common. For selections (1)-(6), we use information from only 
SciBar. At the selection (7), we use EC to reject muons going though SciBar. At the selection $(8)(9)(10)$, we select two gamma rays from $\pi^{0}$ s and reject gamma ray entering from the outside of SciBar. We use standard tracks for selections (1)-(7) since their main purpose is charged current rejection. For selections (8)(9)(10), we use extended tracks since their main purpose is selecting two gamma rays or rejecting gamma rays from the outside of SciBar.

\subsubsection{Number of Tracks}

To find two gamma rays, we select events with at least two tracks whose timing difference is less than 50 ns. Figure 5.5 shows the number of tracks distribution after the selection.

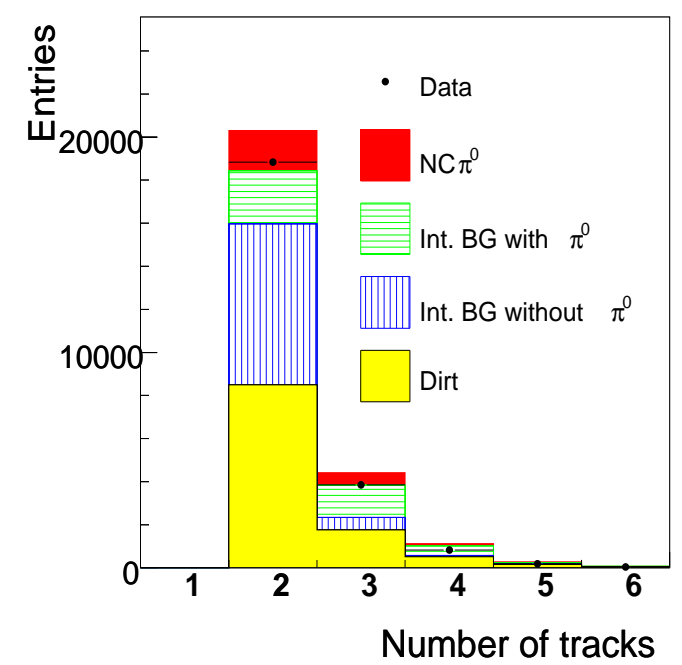

Figure 5.5: The number of tracks distribution; The MC contribution from the $\mathrm{NC} \pi^{0}$, the internal background with $\pi^{0} \mathrm{~s}$ in the final state, the internal background without $\pi^{0} \mathrm{~s}$ in the final state and the dirt background events are shown separately.

\subsubsection{Fiducial Volume Cut}

There are four track edge combinations in two tracks (Figure 5.6). We calculate the distance between edges for these four combination and select the one which gives minimum distance; "Closest edges". In case that two $\gamma_{\mathrm{s}}$ are correctly reconstructed as two tracks, this minimum distance corresponds to the distance between the points where $2 \gamma \mathrm{s}$ convert to the electromagnetic showers. "Closest edges" of two tracks are required to be in this fiducial volume defined in Equation 5.1. Figure 5.7 shows the upstream edge of the longest track after this fiducial volume cut. The discrepancy between data and the MC simulation is seen, especially in the events where the tracks start at the upstream of SciBar. According to the MC simulation, the main contribution of such events is the dirt background events which is not precisely predicted. 


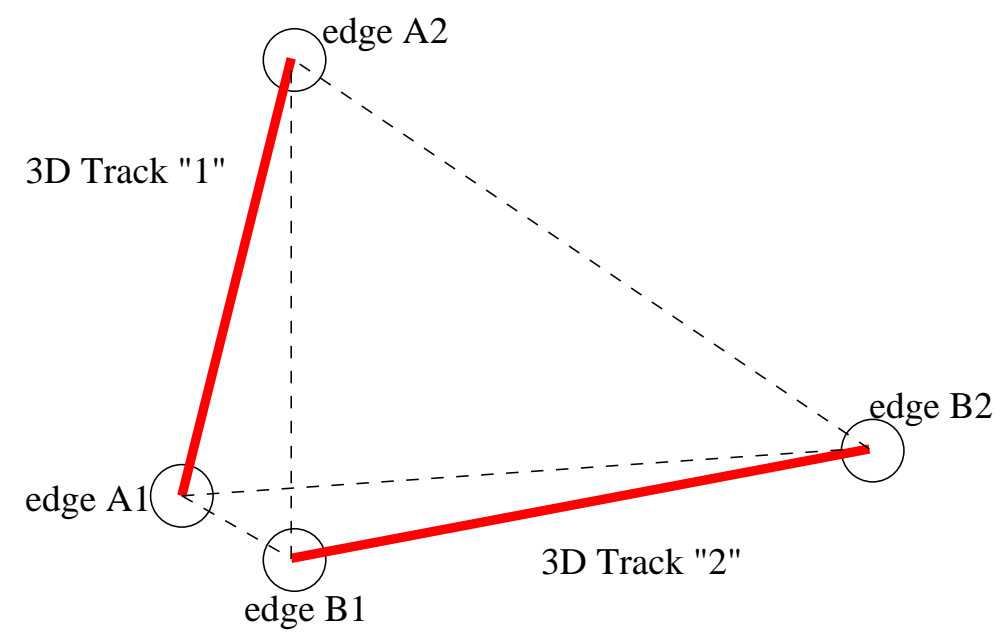

Figure 5.6: There are four edge combinations, which are $(A 1, B 1),(A 1, B 2),(A 2, B 1)$, and $(A 2, B 2)$. And "Closest edges" are A1 and B1.
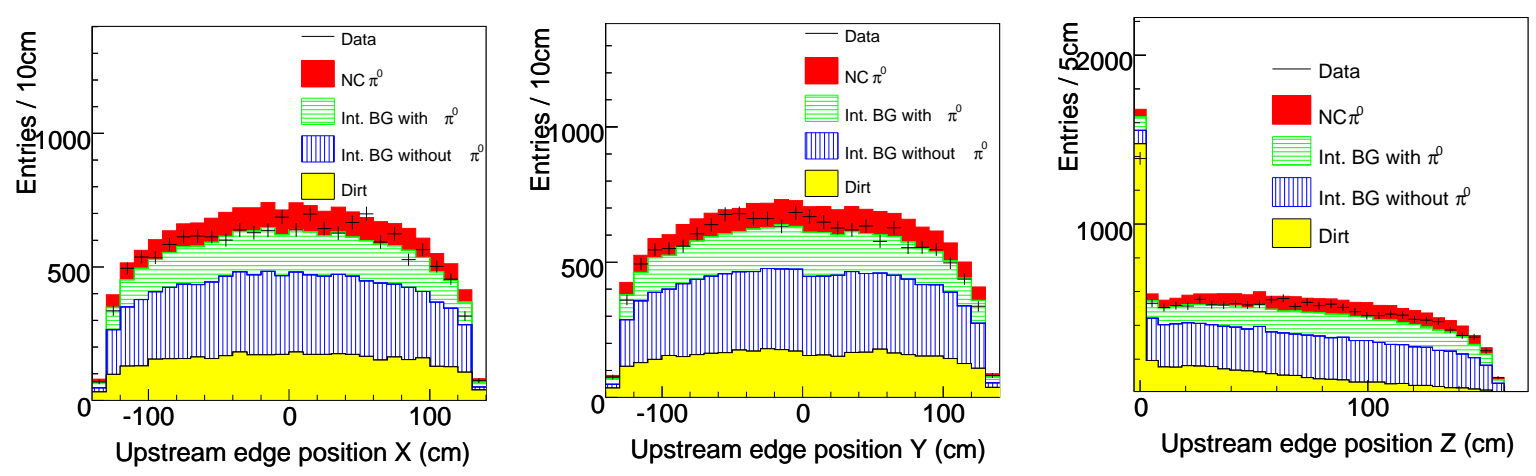

Figure 5.7: The upstream edge distribution of the longest track after this fiducial volume cut

\subsubsection{First Layer Veto}

To reduce dirt background, we reject events with hits in the first layer within $100 \mathrm{~ns}$ from the timing of tracks. Figure 5.8 shows the upstream edge of the longest track after the first layer veto. Figure 5.9 shows the downstream edge of the longest tracks. The peak seen at $z=165 \mathrm{~cm}$ indicates that many muons (charged current events) go out from SciBar.

\subsubsection{Side Escaping Track Rejection Cut}

There are charged current events with muons escaping from the side of SciBar. To reduce these events, the $\mathrm{x}$ and $\mathrm{y}$ position of both edges of the tracks are required to be in fiducial volume (Figure 5.10). Figure 5.11 and 5.12 shows the upstream and downstream edge of the longest tracks after the cut, respectively. By this cut, $41 \%$ of the background events are rejected. Meanwhile, the $\mathrm{NC} \pi^{0}$ efficiency of this cut is $77 \%$. 

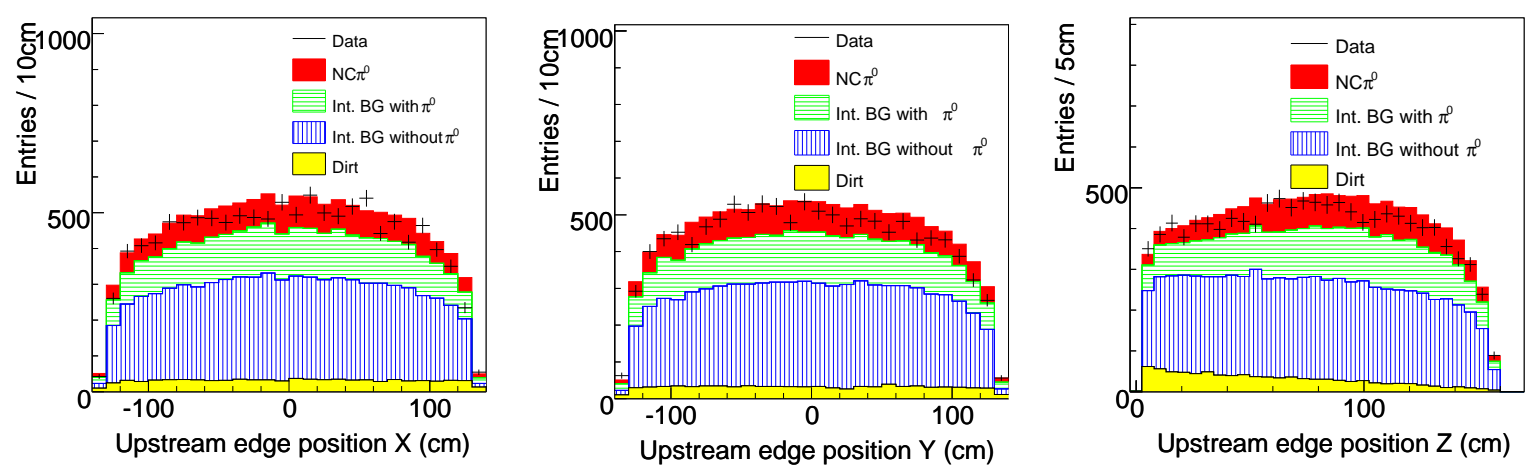

Figure 5.8: The upstream edge distribution of the longest track after first layer veto
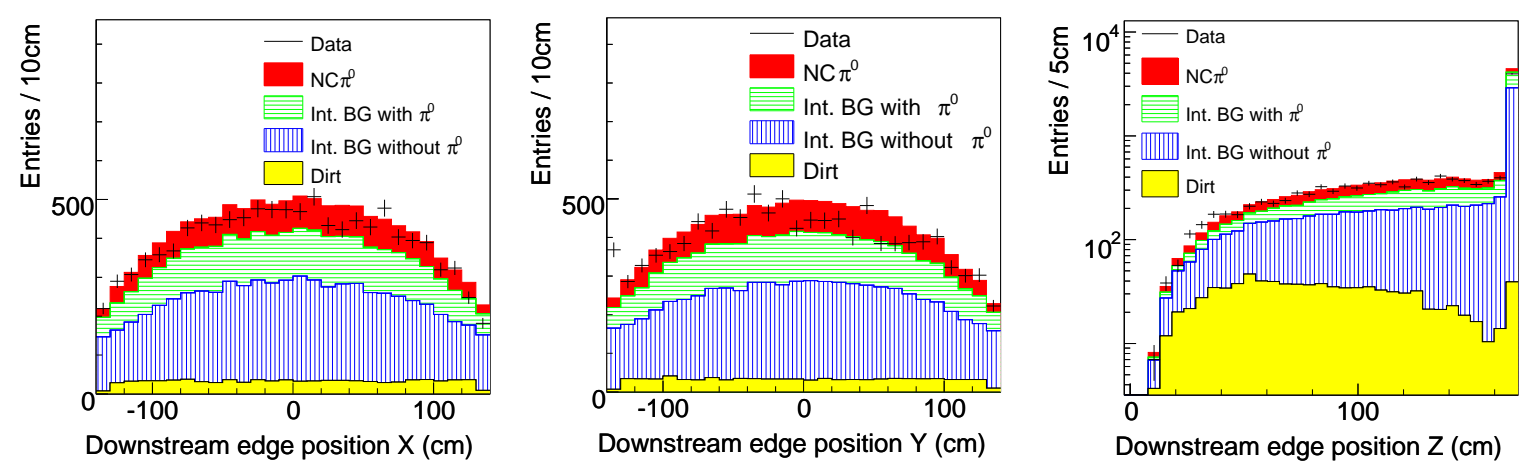

Figure 5.9: The downstream edge distribution of the longest track after the first layer veto

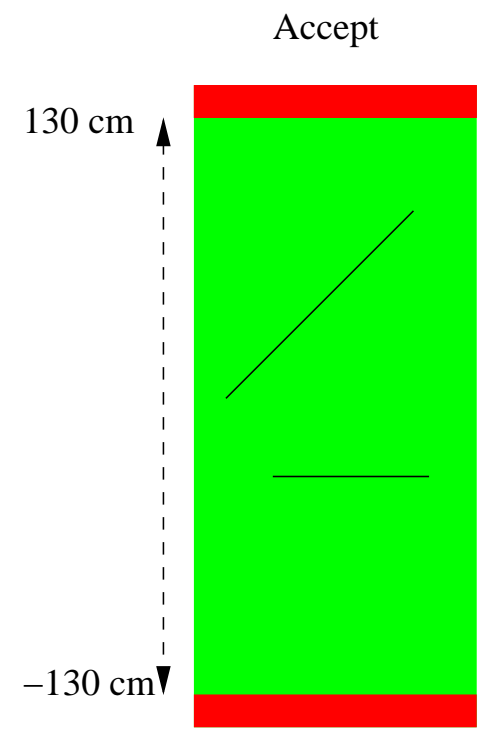

Accept

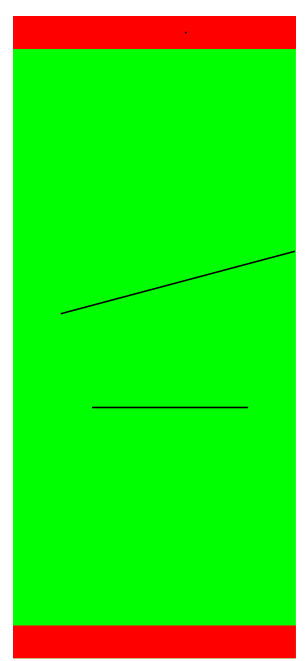

Reject

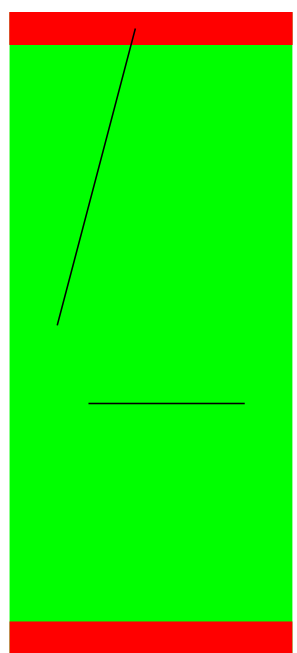

Figure 5.10: Only events with track escaping from the side of SciBar are rejected 

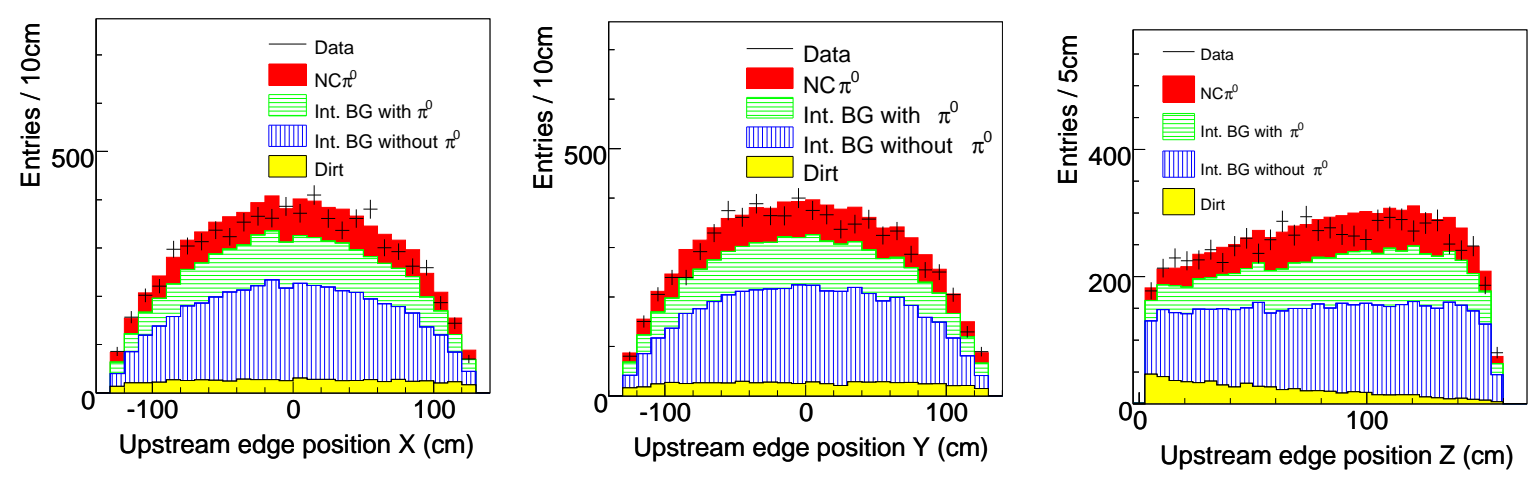

Figure 5.11: The upstream edge of the longest track after rejecting side escaping tracks
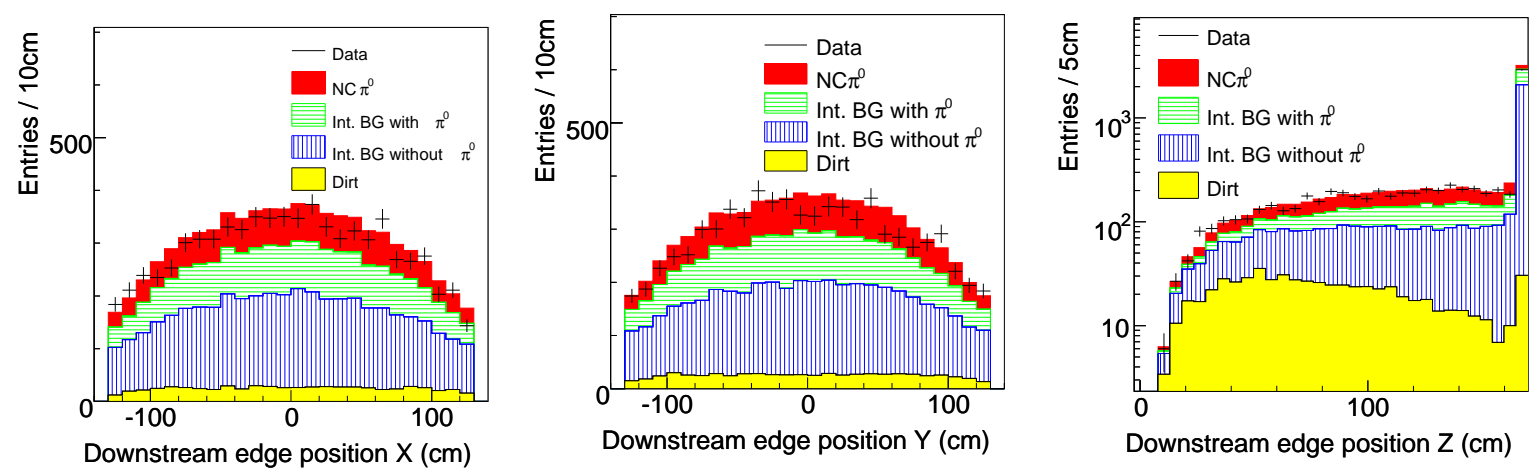

Figure 5.12: The downstream edge of the longest track after rejecting side escaping tracks

\subsubsection{Decay Electron Rejection Cut}

To reject muons stopping in SciBar, we tag the electrons from muon decay. No charge information is recorded for any scintillator strip after the first hit in an event, but the times of hits above threshold are recorded. Thus most decay electrons are not reconstructed as tracks but can be identified as delayed time hits near the end of a muon track. To detect decay electrons, we use a value called $\Delta t_{\text {max }}$ defined as below

1. Find TDC channels corresponding to the upstream and downstream edges of all tracks for both views

2. Among founded TDC channels, choose TDC hits coincident with another view within 50 ns to reduce the accidental noise. Since there are multiple tracks and TDC hits, the number of chosen TDC hits could be more than one.

3. Calculate timing difference between hits at the upstream and downstream. In case of more than one combination of the upstream and downstream hits due to multiple tracks and TDC hits, the difference is calculated for any combinations.

4. Define the maximum one below $5000 \mathrm{~ns}$ as $\Delta t_{\max }$.

Events with decay electrons yield large values of $\Delta t_{\max }$ because of the long muon lifetime $\left(\tau_{\mu}=2.2 \mu \mathrm{sec}\right)$ while $\Delta t_{\max }$ is less than $10 \mathrm{~ns}$ (time that particles transport in SciBar) in case of no decay electron at any edges. Figure 5.13 shows the distribution of $\Delta t_{\max } . \Delta t_{\max }$ of the most of no decay electron events is less than $100 \mathrm{~ns}$. We reject 
events with $\Delta t_{\max }$ greater than 100 ns. By this cut, $31 \%$ of the background events are rejected. Meanwhile, the $\mathrm{NC} \pi^{0}$ efficiency of this cut is $94 \%$.

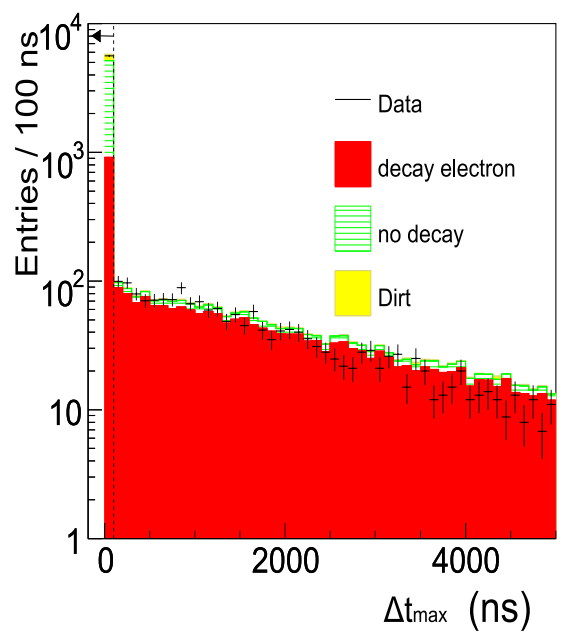

Figure 5.13: Distribution of $\Delta t_{\max }$; The MC contribution from events with decay electrons in SciBar, events without decay electrons in SciBar and dirt backgrounds, are shown separately.

\subsubsection{Track Disconnection Cut}

Charged current events often have multiple tracks with a common vertex while $2 \gamma \mathrm{s}$ from $\pi^{0}$ usually are isolated from each other. The distance between tracks is a good value to separate the $2 \gamma$ events from the charged current events. We define "minimum track distance" as below

1. Select two tracks

2. Calculate 2D distances for all combination of edges of two tracks.

3. Repeat the same procedure for all combinations of two tracks. We have several 2D distances.

4. Define the minimum one as a "minimum track distance"

Figure 5.14 shows the "minimum track distance". The contamination of charged current events concentrates around zero. We reject events with the minimum track distance less than $6 \mathrm{~cm}$.

\subsubsection{Electron Catcher Cut}

Matched EC clusters are used to reject muons penetrating the EC. Two quantities are used: the energy deposit in matched EC clusters in the upstream layer, $E_{1}$, and the ratio of energy deposits in the downstream EC cluster $\left(E_{2}\right)$ over the upstream EC 

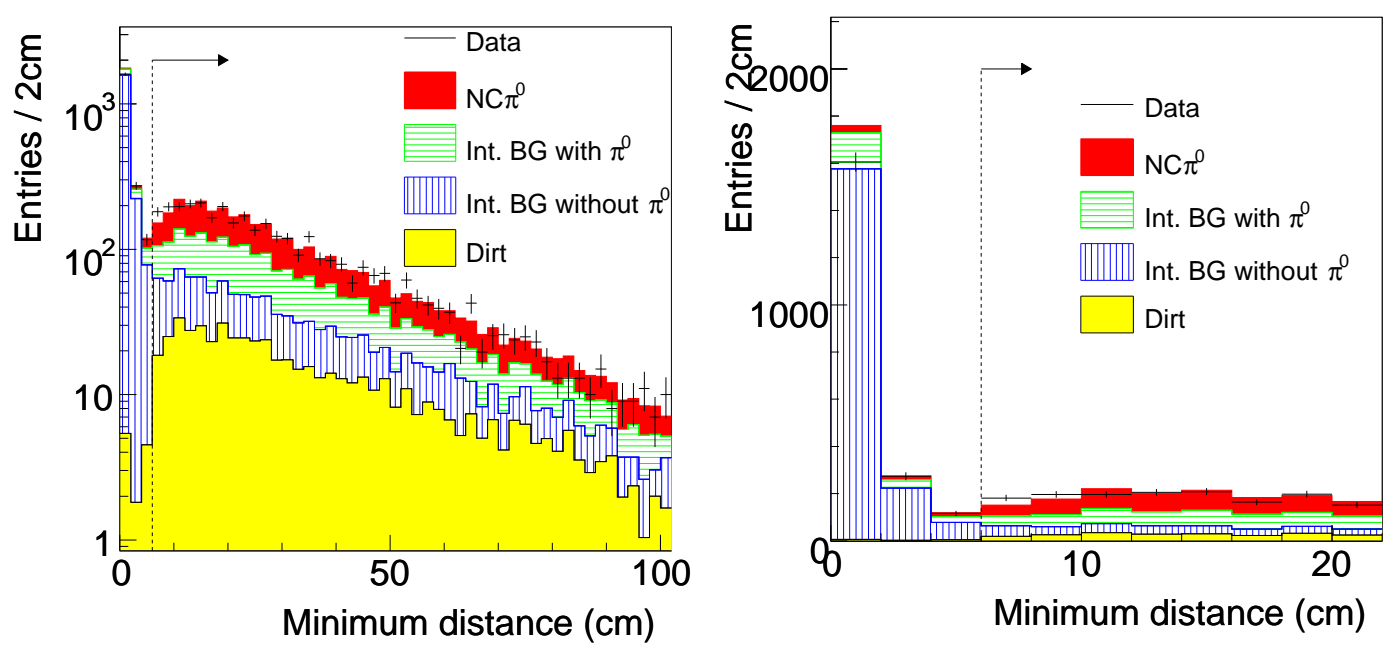

Figure 5.14: Distribution of "minimum track distance" after decay electron rejection. The right plot is a zoomed-out version of the left plot

cluster, $\mathrm{R}_{\mathrm{EC}}=E_{2} / E_{1}$. Since muons tend to penetrate more material than $\gamma_{\mathrm{s}}$, the energy deposit of muon at both upstream and downstream planes are close to each other while $\gamma_{\mathrm{S}}$ stop in the short range after conversion with large energy deposit in the upstream cluster. Figure 5.15 shows scatter plots between $E_{1}$ and $R_{\mathrm{EC}}$ for muons and $\gamma \mathrm{s}$ in the MC simulation. The $E_{1}$ and $\mathrm{R}_{\mathrm{EC}}$ are defined event by event as described below.
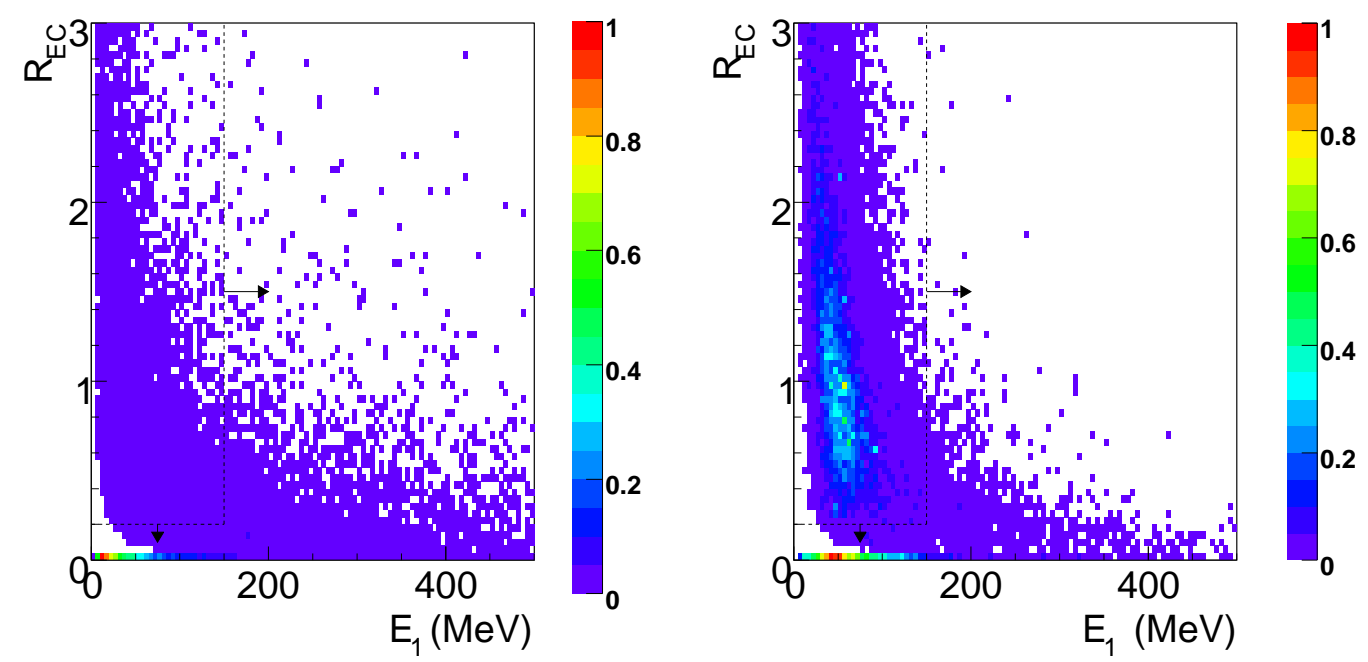

Figure 5.15: The 2D plots between the $E_{1}$ and $\mathrm{R}_{\mathrm{EC}}$ for $\gamma \mathrm{s}$ (left) and muons (right). Events without any tracks matched to EC clusters are not included in these plots.

1. For events without any tracks matched to EC clusters, both $E_{1}$ and $\mathrm{R}_{\mathrm{EC}}$ are left undefined.

2. For events with tracks matched with both upstream and downstream EC clusters, 
the track with maximum energy ratio of the upstream plane to the downstream plane is selected. The maximum energy ratio is $\mathrm{R}_{\mathrm{EC}}$. The upstream energy deposit in the track is $E_{1}$.

3. For events with only tracks matched with upstream EC cluster, the track giving minimum upstream energy deposit is selected as $E_{1}$. $\mathrm{R}_{\mathrm{EC}}$ is 0 .

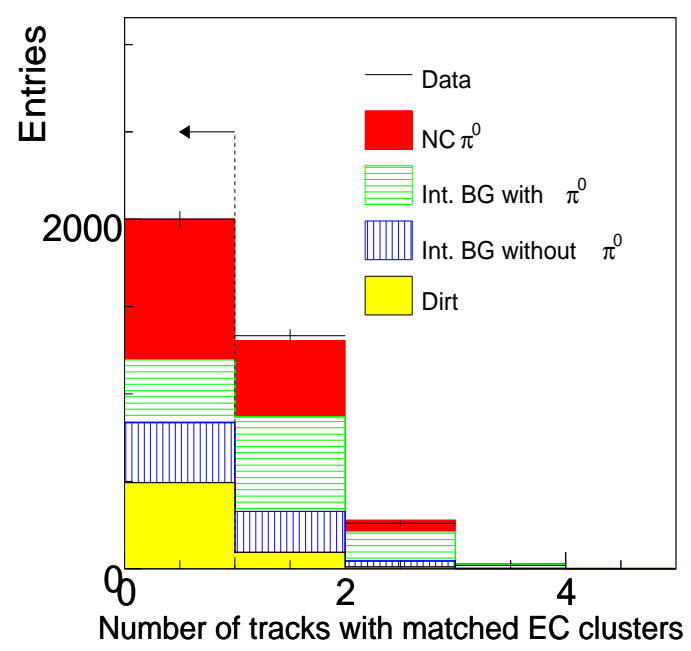

Figure 5.16: Option 1 of the EC cut: the number of track with matched EC clusters after the track disconnection cut. Events without SciBar-EC matched tracks pass the EC cut. Events with SciBar tracks matching EC clusters can pass the EC cut if they satisfy the Option 2 or Option 3.

Figure 5.16, 5.17 and 5.18 show the number of tracks with matched EC clusters, $E_{1}$ and $\mathrm{R}_{\mathrm{EC}}$. Events satisfying at least one of three requirements below are selected

Option 1 There are no tracks matched to the EC clusters.

Option $2 E_{1}$ is greater than $150 \mathrm{MeV}$

Option $3 \mathrm{R}_{\mathrm{EC}}$ is less than 0.2

By this cut, $28 \%$ of the background events are rejected. Meanwhile, the $\mathrm{NC} \pi^{0}$ efficiency of this cut is $91 \%$.

\subsubsection{The Number of Extended Tracks}

After the EC cut, we reconstruct $\gamma_{\mathrm{s}}$ to select $\pi^{0}$ events and reject $\gamma_{\mathrm{s}}$ from the outside of SciBar. Therefore our event selections are based on "extended track" rather than the standard track. As described in Section 5.3.4 the methods for extended tracks are applied for non-proton-like tracks. Figure 5.19 shows the MuCL distribution after the EC cut. The dashed line $(\mathrm{MuCL}=0.03)$ separates particles to proton-like or non-proton-like tracks. The gamma ray efficiency for the non-proton-like sample is $87 \%$ and the purity 


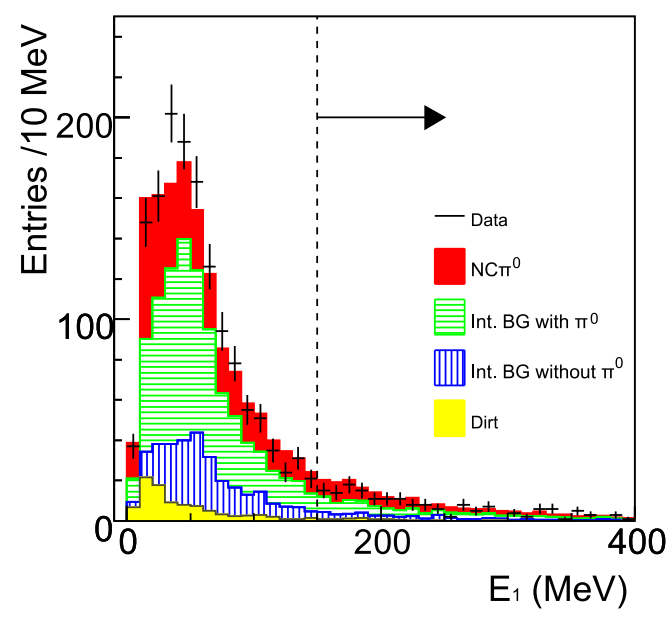

Figure 5.17: Option 2 of the EC cut: the energy deposited in the upstream layer of the EC $\left(E_{1}\right)$ after the track disconnection cut. Events with $E_{1}>150 \mathrm{MeV}$ pass the EC cut. Events with $E_{1}<150 \mathrm{MeV}$ can pass the EC cut if they satisfy the Option 3.

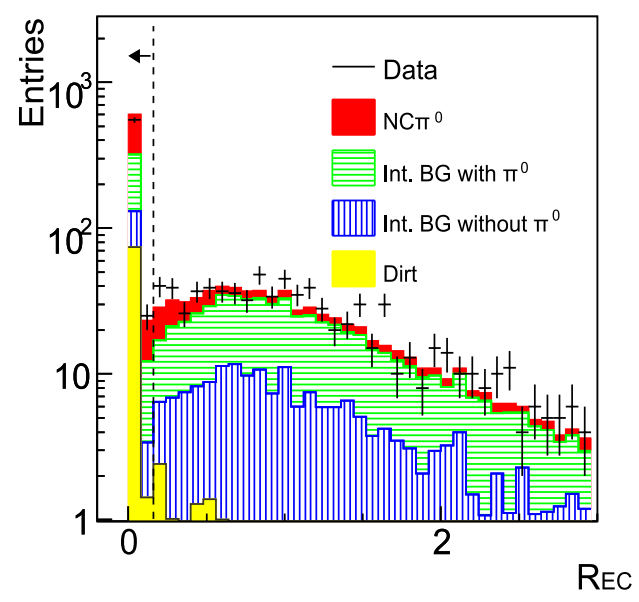

Figure 5.18: Option 3 of the EC cut: the ratio of deposited energy in the downstream over the upstream layer $(\mathrm{R})$ after the track disconnection cut. Only events with $E_{1}<150 \mathrm{MeV}$ are shown.

of gamma rays in the non-proton-like sample is $81 \%$. Figure 5.20 shows the number of non-proton-like tracks and proton-like tracks.

The contribution from the dirt backgrounds is not negligible after EC cut. About $70 \%$ of two tracks in dirt background events are made by single $\gamma$ from $\pi^{0}$ produced in the outside of the detectors. Since two tracks made by the single $\gamma$ are nearly co-linear, these tracks become just one extended track by the track merging. Figure 5.21 shows the number of extended tracks. Many dirt backgrounds are just one extended track as expected. Events with at least two extended tracks are selected for $\pi^{0}$ reconstruction. 


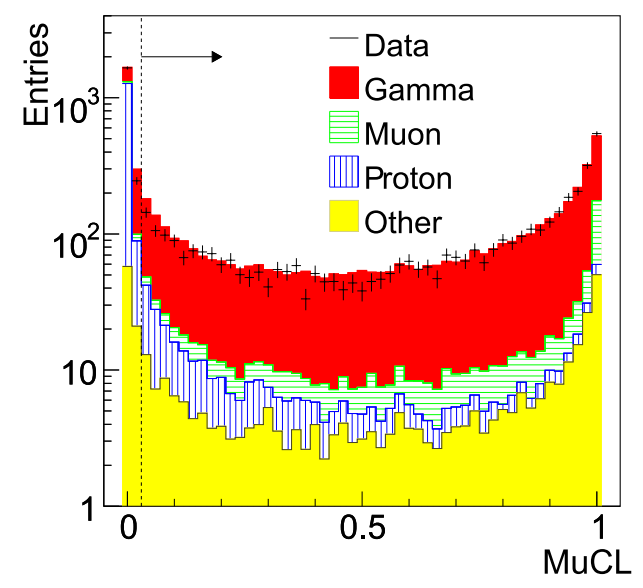

Figure 5.19: Distribution of MuCL of tracks after the EC cut.
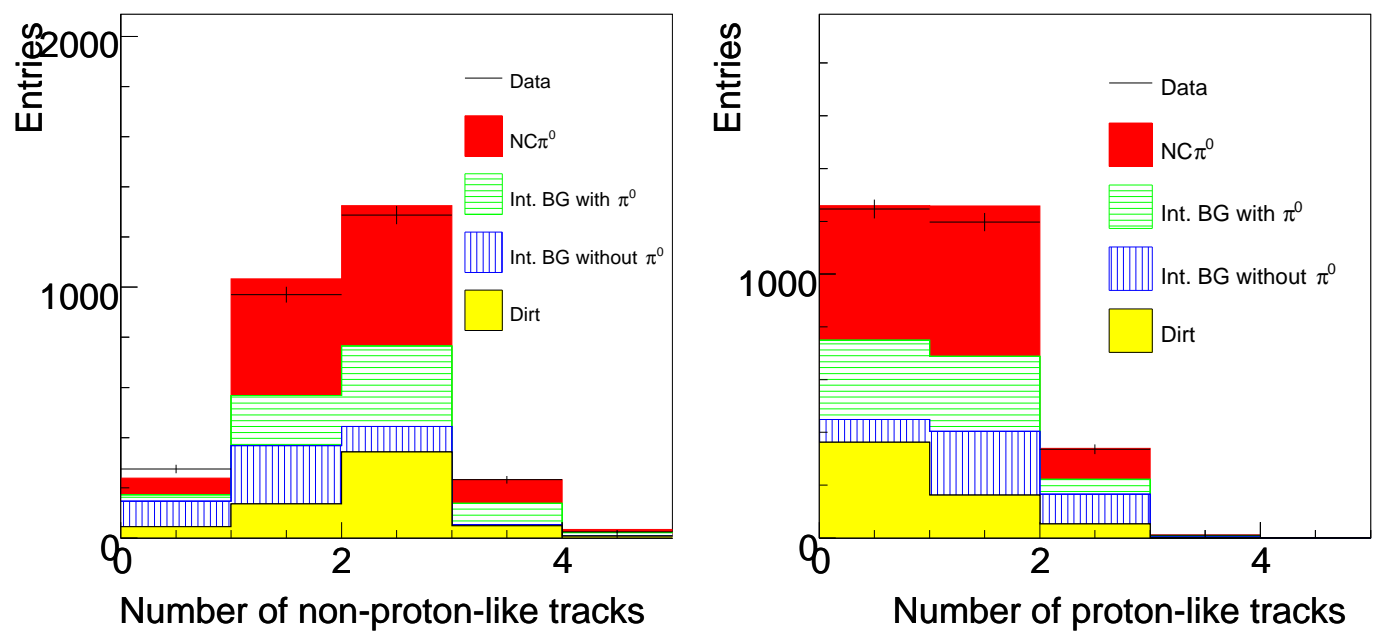

Figure 5.20: From the left, the number of non-proton-like tracks and that of proton-like tracks, respectively

As shown in Figure 5.21, $58 \%$ of the $\mathrm{NC} \pi^{0}$ signals have only one extended track and rejected by the cut. However, $67 \%$ among such rejected $\mathrm{NC} \pi^{0}$ signals are events with one reconstructed gamma ray, due to mis-reconstruction or gamma rays not converting in SciBar. Such events can not be used for $\pi^{0}$ reconstruction; $21 \%$ are events where the two gamma rays are reconstructed as two tracks but one of them is identified as a proton-like track; $12 \%$ are events in which the two gamma rays are reconstructed as two tracks but then merged, resulting in one extended track.

\subsubsection{0 $\pi^{0}$ Vertex Cut}

As described in Section 5.5.9, the events with single $\gamma$ from the outside of the detectors are main contribution to dirt background events and such dirt background events are 


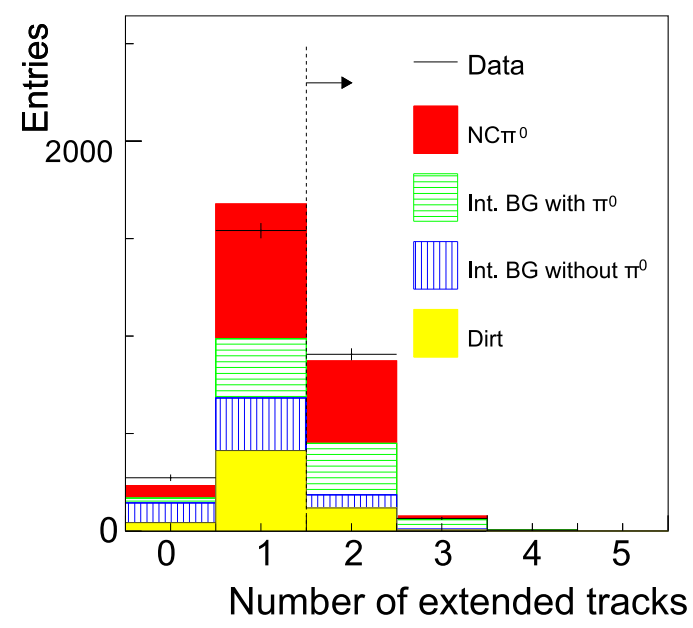

Figure 5.21: The number of extended tracks after the EC cut

rejected by selecting events with at least two extended tracks. After selecting events with at least two extended tracks, the dirt background events have $2 \gamma \mathrm{s}$ from $\pi^{0}$. We reject such dirt background events by reconstructing the $\pi^{0}$ vertex. We reconstruct $\pi^{0}$ vertex in the following way.

1. Fit extended tracks for both views with linear function as below.

$$
\begin{gathered}
x=\mathrm{a}_{\mathrm{top}} \mathrm{Z}+\mathrm{b}_{\text {top }} \\
y=\mathrm{a}_{\text {side }} \mathrm{Z}+\mathrm{b}_{\text {side }},
\end{gathered}
$$

where $\left(a_{\text {top }}, b_{\text {top }}\right)$ and $\left(a_{\text {side }}, b_{\text {side }}\right)$ are fitting parameters of the linear function for the top view and side view, respectively.

2. Calculate the intersection of two extended tracks for each view. $\left(z_{x}, x\right)$ for top view and $\left(z_{y}, y\right)$ for side view are obtained.

3. Calculate the error of intersection by propagating errors of a,b. $\left(\delta z_{x}, \delta x\right)$ for top view and $\left(\delta z_{y}, \delta y\right)$ for side view are obtained.

4. The $z$-vertex is obtained as error weighted average of $z_{x}$ and $z_{y}$.

$$
z=\frac{\frac{z_{x}}{\delta z_{x}{ }^{2}}+\frac{z_{y}}{\delta z_{y}{ }^{2}}}{\frac{1}{\delta z_{x}{ }^{2}}+\frac{1}{\delta z_{y}{ }^{2}}}
$$

5. In case of more than two extended tracks, the combination of two extended tracks with minimum $\left|z_{x}-z_{y}\right|$ is chosen.

The $x, y, z$-vertex calculated in this way are shown in Figure 5.22. Since most of the contributions at $z<0 \mathrm{~cm}$ are the dirt background events as expected, events with $\mathrm{z}$ greater than $0 \mathrm{~cm}$ are selected. 

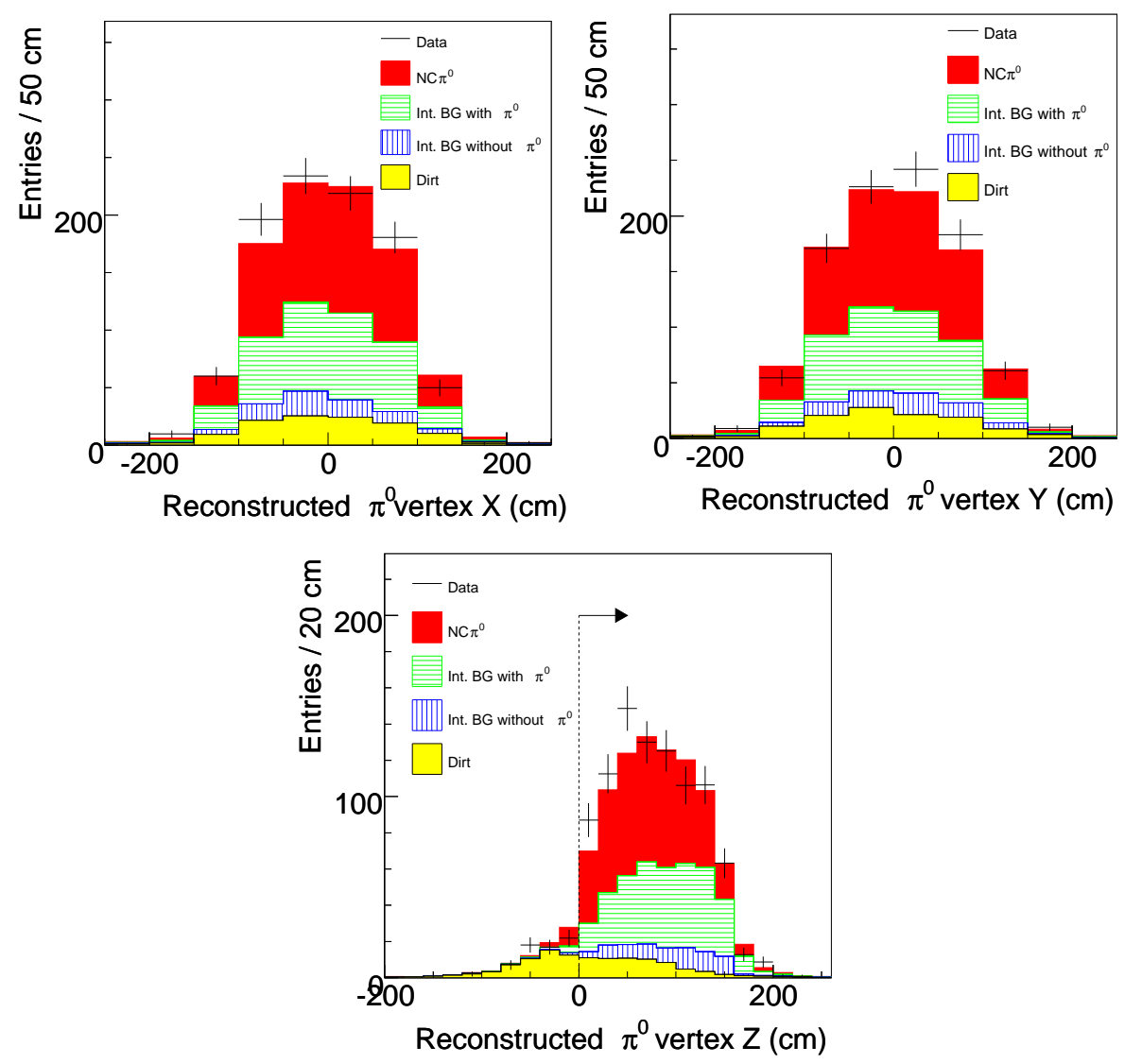

Figure 5.22: Reconstructed $\pi^{0}$ vertex $(x, y, z)$ after the requirement of at least two extended tracks

\subsubsection{1 $\pi^{0}$ Mass Cut}

Figure 5.23 shows the reconstructed $\pi^{0}$ mass $\left(\mathrm{M}_{\pi^{0}}^{\mathrm{rec}}\right)$ calculated as

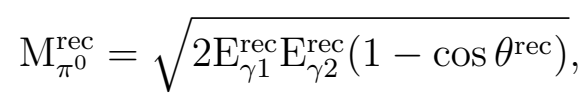

where $E_{\gamma 1}^{r e c}$ and $E_{\gamma 2}^{r e c}$ are the reconstructed energies of two selected extended tracks $\left(E_{\gamma 1}^{\mathrm{rec}}>\mathrm{E}_{\gamma 2}^{\mathrm{rec}}\right)$, and $\theta^{\text {rec }}$ is the angle between two selected extended tracks. To reject dirt backgrounds and charged current events, we select events with $50 \mathrm{MeV} / \mathrm{c}^{2}<\mathrm{M}_{\pi^{0}}^{\mathrm{rec}}<200 \mathrm{MeV} / \mathrm{c}^{2}$. The fact that the peak value is smaller than the actual $\pi^{0}$ mass $(135 \mathrm{MeV})$ is due to energy leakage of $\gamma \mathrm{s}$ described in Appendix A.3.

\subsubsection{Event Summary}

Table 5.1 shows the number of events for data and MC simulation at each event selection stage. We select 657 events in data after all cuts. Subtracting the estimated background of 240 events (202 internal and 38 external) yields 417 signal events. The MC expectation is 368 events. The purity of $\mathrm{NC} \pi^{0}$ production after all event selection cuts is estimated to be $61 \%$ (40\% from single $\pi$ production via resonance decay, $15 \%$ from coherent $\pi$ production and $5 \%$ from neutrino deep inelastic scattering). According to our 


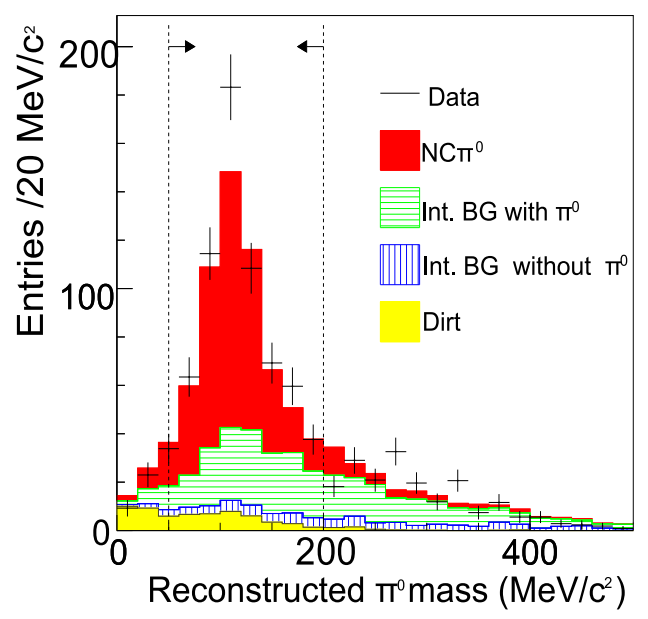

Figure 5.23: Reconstructed $\pi^{0}$ mass

MC simulation, $96 \%$ of selected $\mathrm{NC} \pi^{0}$ events have one $\pi^{0}$ (91 \% from a single $\pi^{0}$ without any other mesons and $5 \%$ from a single $\pi^{0}$ with charged mesons) and $4 \%$ have two $\pi^{0}$ s. The efficiency for $\mathrm{NC} \pi^{0}$ events is defined as:

$$
\epsilon_{\mathrm{NC} \pi^{0}}=\frac{\text { the number of selected NC } \pi^{0} \text { events }}{\text { the number of generated } \mathrm{NC} \pi^{0} \text { events in the SciBar FV }}
$$

The efficiency is $5.3 \%$. The internal background, which accounts for $33 \%$ of this sample, contains charged current $\pi^{0}$ production including secondary $\pi^{0} \mathrm{~s}(18 \%)$, neutral current secondary $\pi^{0}$ production in detector materials (9\%) and non- $\pi^{0}$ background (6\%). According to our MC simulation, the average energy of neutrinos producing $\mathrm{NC} \pi^{0}$ events in the SciBar fiducial volume is $1.3 \mathrm{GeV}$ and the average energy of neutrinos producing $\mathrm{NC} \pi^{0}$ events that pass all selection cuts is $1.1 \mathrm{GeV}$.

\begin{tabular}{|c||c|c|c|c||c|c||c|c|}
\hline $\begin{array}{c}\text { event } \\
\text { selection }\end{array}$ & $\begin{array}{c}\text { NC } \\
\pi^{0}\end{array}$ & $\begin{array}{c}\text { Int. BG } \\
\text { with } \pi^{0}\end{array}$ & $\begin{array}{c}\text { Int. BG } \\
\text { without } \pi^{0}\end{array}$ & $\begin{array}{c}\text { Dirt } \\
\text { BG }\end{array}$ & $\begin{array}{c}\text { MC } \\
\text { total }\end{array}$ & Data & $\begin{array}{c}\text { NC } \pi^{0} \\
\text { Efficiency }\end{array}$ & $\begin{array}{c}\text { NC } \pi^{0} \\
\text { Purity }\end{array}$ \\
\hline \hline 2 tracks & 2578 & 4516 & 8120 & 10970 & 26183 & 23761 & $37.1 \%$ & $10 \%$ \\
FV & 2142 & 3623 & 7034 & 4160 & 16960 & 15336 & $30.9 \%$ & $13 \%$ \\
1st layer veto & 1893 & 3086 & 6722 & 895 & 12596 & 11926 & $27.3 \%$ & $15 \%$ \\
No side escape & 1465 & 1947 & 3760 & 638 & 7810 & 7444 & $21.1 \%$ & $19 \%$ \\
Decay-e cut & 1377 & 1302 & 2483 & 606 & 5768 & 5609 & $19.8 \%$ & $24 \%$ \\
Trk disconnection & 1314 & 1089 & 617 & 595 & 3614 & 3614 & $18.9 \%$ & $36 \%$ \\
EC cut & 1202 & 645 & 443 & 579 & 2870 & 2791 & $17.3 \%$ & $42 \%$ \\
2 extracks & 444 & 318 & 72 & 121 & 955 & 973 & $6.5 \%$ & $46 \%$ \\
$\pi^{0}$ vtx cut & 428 & 311 & 70 & 65 & 874 & 904 & $6.2 \%$ & $49 \%$ \\
$\pi^{0}$ mass cut & 368 & 174 & 29 & 38 & 608 & 657 & $5.3 \%$ & $61 \%$ \\
\hline
\end{tabular}

Table 5.1: The number of events for data and MC at each event selection stage. 


\section{Chapter 6}

\section{Study of Neutral Current $\pi^{0}$ Production}

\subsection{Overview}

There are three main goals for this analysis. The first is to measure the cross section of $\mathrm{NC} \pi^{0}$ production. We measure the ratio of $\mathrm{NC} \pi^{0}$ cross section to the total charged current cross section in order to minimize the systematic uncertainty of the neutrino flux prediction. The second goal is to measure the $\pi^{0}$ kinematics which are momentum and angular distribution. The last one is to measure the coherent $\pi^{0}$ contribution in the context of the Rein and Sehgal model.

In this Chapter, we measure the cross section ratio in Section 6.2, and the $\pi^{0}$ kinematics using selected events in Section 6.3. We also present measurement of the coherent $\pi^{0}$ contribution in Section 6.4. Finally, we summarize all results in this thesis in Section 6.5. At the end, we discuss the possible improvement of the analyses and future prospects in Section 6.6

\subsection{Neutral Current $\pi^{0}$ Cross Section}

We measure the cross section ratio of the neutral current $\pi^{0}$ production to the total charged current interaction.

\subsubsection{Neutral Current $\pi^{0}$ Production}

The efficiency corrected number of neutral current $\pi^{0}$ events is calculated as

$$
N\left(\mathrm{NC} \pi^{0}\right)=\frac{N_{\mathrm{obs}}-N_{\mathrm{BG}}}{\epsilon_{\mathrm{NC} \pi^{0}}}
$$

where $N_{\text {obs }}$ is the number of observed events, $N_{\mathrm{BG}}$ is the number of background events estimated with the MC simulation, and $\epsilon_{\mathrm{NC} \pi^{0}}$ is the selection efficiency of neutral current $\pi^{0}$ events defined as Equation 5.6. $N_{\text {obs }}$ and $N_{\mathrm{BG}}, \epsilon_{\mathrm{NC} \pi^{0}}$ are 657, 240.0 and 0.053, respectively. After subtracting background and correcting for the selection efficiency, the number of neutral current $\pi^{0}$ events is measured to be $[7.8 \pm 0.5$ (stat.) $] \times 10^{3}$. The neutrino 
energy dependence of the selection efficiency for neutral current $\pi^{0}$ events is shown in Figure 6.1. The mean neutrino beam energy for true neutral current $\pi^{0}$ events in the sample is estimated to be $1.14 \mathrm{GeV}$ after accounting for the effects of the selection efficiency.
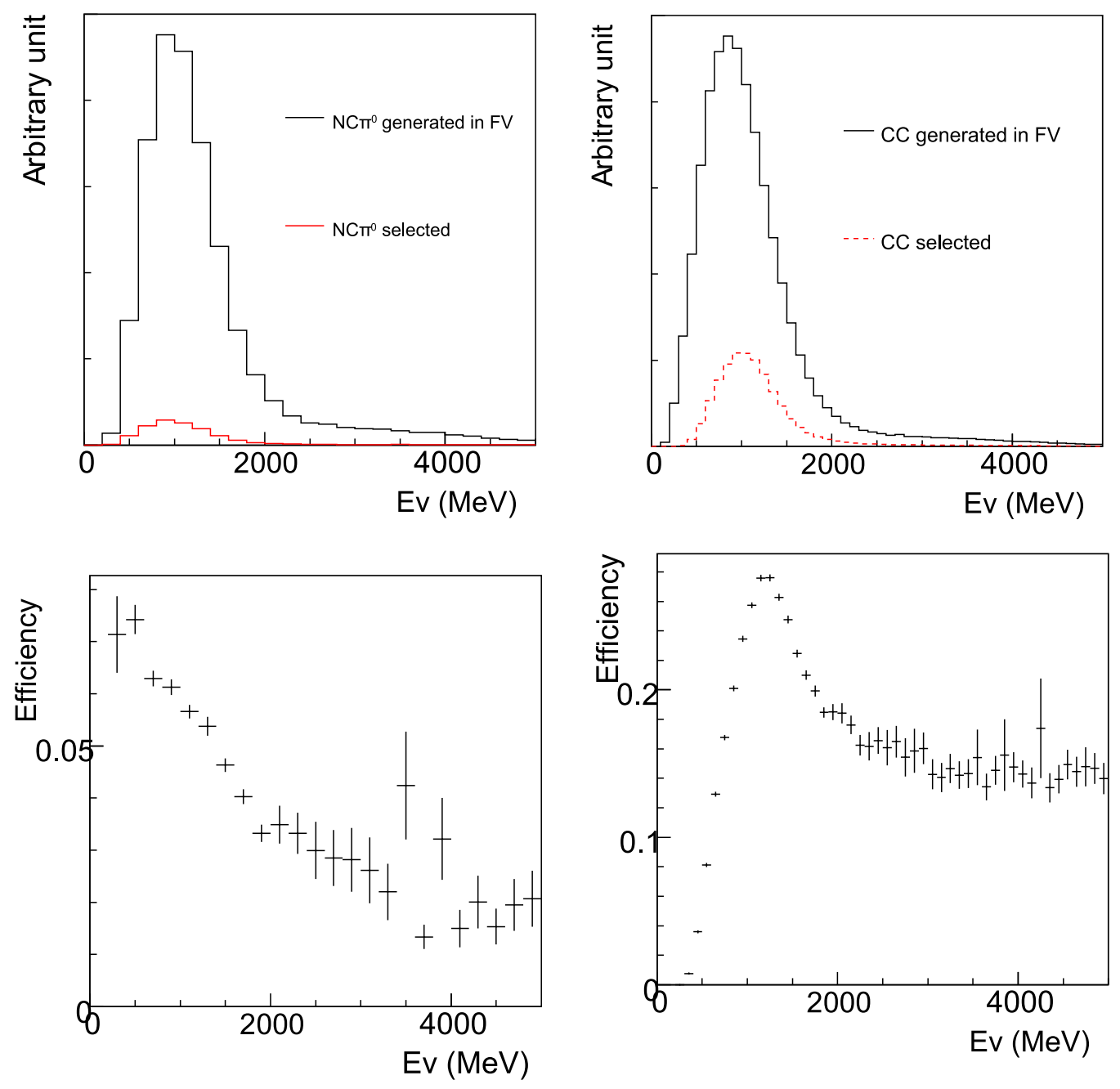

Figure 6.1: Neutrino energy spectra and selection efficiency as a function of neutrino energy for neutral current $\pi^{0}$ events (left) and all charged current events (right).

\subsubsection{Total Charged Current Interaction}

The total number of charged current interaction is estimated by using the SciBarMRD stopped sample. The number of charged current candidates after correcting for the selection efficiency is calculated as

$$
N(\mathrm{CC})=\frac{N_{\mathrm{obs}}^{\mathrm{CC}}-N_{\mathrm{BG}}^{\mathrm{CC}}}{\epsilon_{\mathrm{CC}}}
$$


where $N_{\mathrm{obs}}^{\mathrm{CC}}$ is the number of observed charged current events, $N_{\mathrm{BG}}^{\mathrm{CC}}$ and $\epsilon_{\mathrm{CC}}$ are the number of background events and selection efficiency in the sample, respectively, estimated by the MC simulation. We observed 21,702 MRD-stopped events $\left(N_{\text {obs }}^{C C}\right)$. The number of background events and the selection efficiency are estimated to be $2348\left(N_{\mathrm{BG}}^{\mathrm{CC}}\right)$ and $19 \%\left(\epsilon_{\mathrm{CC}}\right)$, respectively. The neutrino energy dependence of the selection efficiency for charged current events is shown in Figure 6.1. After subtracting the background events and correcting for the efficiency, the number of charged current events is measured to be $[1.02 \pm 0.01$ (stat. $)] \times 10^{5}$.

\subsubsection{Cross Section Ratio}

The ratio of the neutral current $\pi^{0}$ production to the total charged current cross section is measured to be

$$
\begin{aligned}
\frac{\sigma\left(\mathrm{NC} \pi^{0}\right)}{\sigma(\mathrm{CC})} & =\frac{N\left(\mathrm{NC} \pi^{0}\right)}{N(\mathrm{CC})} \\
& =(7.7 \pm 0.5(\text { stat. }) \pm 0.5(\text { sys. })) \times 10^{-2}
\end{aligned}
$$

at the mean neutrino energy of $1.14 \mathrm{GeV}$; systematic uncertainties are described in section 6.2.4. The MC expectation based on the Rein and Sehgal model [18] is $6.8 \times 10^{-2}$. Although the value of this measurement is larger than the expectation by $11 \%$, the measurement is consistent with the expectation of the Rein and Sehgal model within the uncertainty.

\subsubsection{Systematic Uncertainty}

The sources of systematic error are divided into four categories, (i) detector response and track reconstruction, (ii) nuclear effects and neutrino interaction models, (iii) neutrino beam and (iv) dirt background. We vary these sources within their uncertainties and take the resulting change in the cross section ratio as the systematic uncertainty of the measurement. Table 6.1 summarizes the systematic errors in the neutral current $\pi^{0}$ cross section ratio. The total systematic error is $\pm 0.5 \times 10^{-2}$ on the cross section ratio.

\begin{tabular}{ccc}
\hline \hline Source & \multicolumn{2}{c}{ error $\left(\times 10^{-2}\right)$} \\
\hline Detector response & -0.39 & 0.38 \\
$\nu$ interaction & -0.25 & 0.30 \\
Dirt backgrounds & -0.10 & 0.10 \\
$\nu$ beam & -0.11 & 0.22 \\
\hline Total & $-0.5(-0.483)$ & $0.5(0.542)$ \\
\hline \hline
\end{tabular}

Table 6.1: Summary of the systematic errors in the neutral current $\pi^{0}$ cross section ratio. The numbers in the last row show the quadratic summation of numbers in other row with different digit (1 and 3 ) 


\section{Detector Response and Track Reconstruction}

The uncertainties in the crosstalk and the resolution of the MA-PMT,the scintillator quenching effect, simulation of pion interaction in SciBar, the hit threshold for the track reconstruction, the $\gamma$ reconstruction and the energy scale are considered. The systematic errors for each source in this category are in Table 6.2.

Crosstalk and the Resolution of MA-PMT The crosstalk of the MA-PMT was measured to be $3.15 \%$ for adjacent channels, with an absolute error of $0.4 \%$. The single photoelectron resolution of the MA-PMT is set to $50 \%$ in the simulation, and the absolute error is estimated to be $\pm 20 \%$. We prepare several MC event sets by changing the cross talk level and single photoelectron resolution separately, and take the change of the cross section ratio as the systematic error.

Scintillator Quenching Birk's constant of the SciBar scintillator was measured to be $0.0208 \pm 0.0023 \mathrm{~cm} / \mathrm{MeV}[54]$ and is varied within the measurement to evaluate the systematic error.

Pion Interaction in SciBar A $10 \%$ difference of the total pion-carbon cross section between the GENAT4 simulation and external measurements is seen for higher energy pions. To evaluate the systematic error due to this effect, we vary the cross section by $\pm 10 \%$, and take the change as the systematic error.

Hit Threshold The conversion factors from the ADC counts to the photoelectron were measured for all 14336 MA-PMT channels. The measurement was at the $20 \%$ level. Since we use the number of photoelectrons (2.5 p.e.) for the hit threshold, the hit threshold for track reconstruction is varied by $\pm 20 \%$ to evaluate the systematic error.

Reconstructed Gamma Direction For the uncertainty on reconstruction of the gamma ray direction, we study how the difference between data and MC distributions changes when we change the gamma direction reconstruction algorithm. We compare our standard algorithm with gamma direction reconstruction obtained using extended tracks - resulting in poorer angular resolution. We take this change as the uncertainty.

EC Energy Scale EC energy information is used for the event selection. The uncertainty of the EC energy scale for $\gamma$ is estimated to be $10 \%$. We vary the reconstructed EC energy by $\pm 10 \%$. The EC energy scale in MC simulation and take the change as systematic uncertainty.

Total Gamma Energy Scale The $\pi^{0}$ mass peak in Figure 6.3 shows good agreement between data and the MC simulation in term of energy scale. We fit the peak of data with a gaussian function. The discrepancy of the gaussian mean between data and the MC simulation is $(2.0 \pm 1.5) \%$. Meanwhile, the discrepancy of the muon energy deposit between data and the MC simulation is $1.3 \%$. Although both Data/MC discrepancy of gamma rays and muons agrees with each other within the error, the discrepancy of gamma rays could be larger than that of muons. This is because the hit distributions 
of gamma ray's tracks are more complicated than that of muons. Therefore we choose 3 $\%$ (larger than $1.3 \%$ but within $2.0 \pm 1.5$ ) for the uncertainty of the gamma ray energy scale. We vary by $3 \%$ reconstructed energy of $\gamma$ and take the variation as the systematic uncertainty.

\begin{tabular}{ccc}
\hline \hline Source & \multicolumn{2}{c}{ error $\left(\times 10^{-2}\right)$} \\
\hline Crosstalk & 0.00 & 0.27 \\
Resolution of MA-PMT & -0.03 & 0.00 \\
Scintillator quenching & 0.00 & 0.17 \\
Pion interaction in SciBar & -0.16 & 0.07 \\
Hit threshold & -0.3 & 0.05 \\
$\gamma$ reconstruction & -0.13 & 0.13 \\
EC energy scale & -0.03 & 0.02 \\
$\gamma$ energy scale & -0.20 & 0.12 \\
Subtotal & $-0.4(0.386)$ & $0.4(0.379)$ \\
\hline \hline
\end{tabular}

Table 6.2: Summary of the systematic errors by the uncertainties in the detector response for the neutral current $\pi^{0}$ cross section ratio. The numbers in the last row show the quadratic summation of numbers in other row with different digit (1 and 3)

\section{Neutrino Interaction Models and Nuclear Effects}

We consider uncertainties in the CC resonant $\pi$ cross section, the NC/CC ratio, the axial vector mass and $\pi$ interaction in the initial target nucleus. The systematic errors for each source in this category are in Table 6.3

CC resonant Pion Cross Section The largest background are CC resonant pion interaction. The uncertainty of the cross section is estimated to be $\pm 20 \%$ based on the K2K measurement [96]. We vary the cross section of charged current resonant pion production by $\pm 20 \%$ and take that change as the systematic error.

NC/CC Ratio The uncertainty of the ratio between NC events to CC events is estimated to be $\pm 20 \%$. We vary the $\mathrm{NC} / \mathrm{CC}$ ratio by $\pm 20 \%$ and take that change as the systematic error.

Axial Vector Mass for QE and Resonant $\pi$ In the NEUT simulation, we set the axial vector mass $M_{A}$ to $1.21 \mathrm{GeV} / c^{2}$ for both $\mathrm{QE}$ and resonant pion production. The uncertainty in this value is estimated to be $\pm 0.1 \mathrm{GeV} / c^{0}$ based on recent measurements $[66,67]$. Since the results from past experiments are systematically lower than the recent measurements [97], we only vary $M_{A}$ down to 1.11

Pion Interaction in the Initial Target Nucleus For pions produced by neutrino interactions, uncertainties on the cross sections for pion absorption and pion inelastic scattering in the nucleus are considered. In the momentum range of pions from $\Delta$ decays, the cross section measurement uncertainty for absorption, inelastic scattering and charge exchange is $30 \%$. 


\begin{tabular}{ccc}
\hline \hline Source & \multicolumn{2}{c}{ error $\left(\times 10^{-2}\right)$} \\
\hline CC resonant pion cross section & -0.14 & 0.16 \\
NC/CC ratio & -0.11 & 0.16 \\
Axial vector mass & 0.00 & 0.07 \\
Pion absorption cross section & -0.17 & 0.19 \\
Pion inelastic cross section & -0.01 & 0.03 \\
Pion charge exchange cross section & -0.03 & 0.04 \\
\hline Subtotal & $-0.3(-0.251)$ & $0.3(0.302)$ \\
\hline \hline
\end{tabular}

Table 6.3: Summary of the systematic errors by the uncertainties in the neutrino interaction models and nuclear effect in the neutral current $\pi^{0}$ cross section ratio. The numbers in the last row show the quadratic summation of numbers in other row with different digit (1 and 3)

\section{Dirt Backgrounds}

As shown in Figure 5.22, the dirt background simulation describes data at $z<-20 \mathrm{~cm}$ where the dirt background is the dominant contamination. However, the statistical uncertainty is large, $15 \%$. We scale the dirt contamination by $\pm 15 \%$ in the final sample and take the change as the systematic error due to dirt backgrounds. As a result, we assign $\pm 0.1 \times 10^{-2}$ on the cross section ratio.

\section{Neutrino Beam}

The uncertainties in the secondary hadron production cross sections in proton-beryllium interactions, hadronic interactions in the target or horn, and the horn magnetic field model are considered. Uncertainties associated with the delivery of the primary proton beam to the beryllium target and the primary beam optics, which result in the overall normalization uncertainty, are not considered in this analysis since it cancels in the cross section ratio.

The change in the neutrino beam spectrum due to these uncertainties is calculated by drawing random parameter vectors and weighting each event by a factor corresponding to the variation of the yield of the parent meson with the given momentum and angle. The extraction of the neutral current $\pi^{0}$ cross section ratio is repeated for each beam systematic parameter set. The efficiency of neutral current $\pi^{0}$ events, the efficiency and purity of the charged current inclusive samples, and the number of background events are re-calculated using each parameter set. The number of observed events is obtained from data.

Figure 6.2 shows the distribution of the neutral current $\pi^{0}$ cross section ratio obtained by using 1000 neutrino flux predictions. The mean and sigma of the distribution are $7.736 \times 10^{-2}$ and $0.162 \times 10^{-2}$,respectively. Since the result using the default MC simulation is $7.680 \times 10^{-2}$, we assign the systematic error of $(+0.218,-0.105) \times 10^{-2}$. 


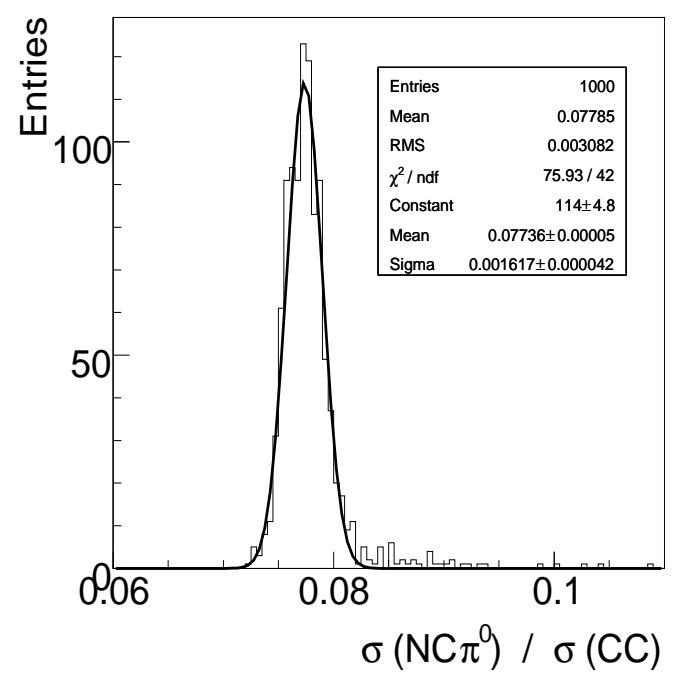

Figure 6.2: The distribution of the neutral current $\pi^{0}$ cross section ratio $\left(\sigma\left(N C \pi^{0}\right) / \sigma(C C)\right)$ obtained by using 1000 neutrino flux predictions.

\subsection{Reconstructed $\pi^{0}$ Kinematics}

In this section, the kinematic valuables of $\pi^{0}$ events are reconstructed. These valuables are very important to check our models describing $\pi^{0}$ production mechanisms and verify that we correctly select and reconstruct the $\pi^{0}$ events.

\subsubsection{Reconstructed $\pi^{0}$ Mass}

Figure 6.3 shows the reconstructed $\pi^{0}$ mass $\mathrm{M}_{\pi^{0}}^{\mathrm{rec}}$ before the $\pi^{0}$ mass cut. The $\mathrm{M}_{\pi^{0}}^{\mathrm{rec}}$ is defined as

$$
\mathrm{M}_{\pi^{0}}^{\mathrm{rec}}=\sqrt{2 \mathrm{E}_{\gamma 1}^{\mathrm{rec}} \mathrm{E}_{\gamma 2}^{\mathrm{rec}}\left(1-\cos \theta^{\mathrm{rec}}\right)}
$$

where $\mathrm{E}_{\gamma 1}^{\mathrm{rec}}$ and $\mathrm{E}_{\gamma 2}^{\mathrm{rec}}$ are the reconstructed energies of two selected extended tracks, and $\theta^{\text {rec }}$ is the angle between two selected extended tracks. The angular resolution of extended tracks is estimated to be 6 degrees by the $\mathrm{MC}$ simulation. The fact that the peak value is smaller than the actual $\pi^{0}$ mass $(135 \mathrm{MeV})$ is due to energy leakage of $\gamma_{\mathrm{S}}$. On average, the reconstructed gamma ray track collect $85 \%$ of the true gamma ray energy according to the MC simulation. The detail performance of the gamma ray reconstruction is discussed in Appendix A.3

The clear peak shows that our $\pi^{0}$ reconstruction is successful. In addition, the MC simulation of the background region (tail of the distribution) describes data well.

We also show the reconstructed energies of the two selected extended tracks $\left(\mathrm{E}_{\gamma 1}^{\mathrm{rec}}>\right.$ $\left.\mathrm{E}_{\gamma 2}^{\mathrm{rec}}\right)$ in Figure 6.4. These plots also show the good agreement between data and the MC simulation. In case of more than two extended tracks, the combination of two extended tracks with minimum $\left|z_{x}-z_{y}\right|$ is chosen as described in Section 5.5.10. 


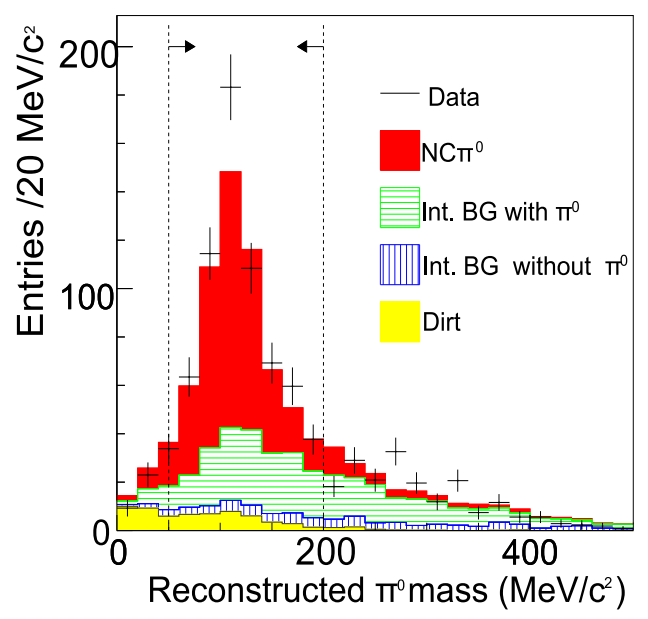

Figure 6.3: Reconstructed $\pi^{0}$ mass before the $\pi^{0}$ mass cut
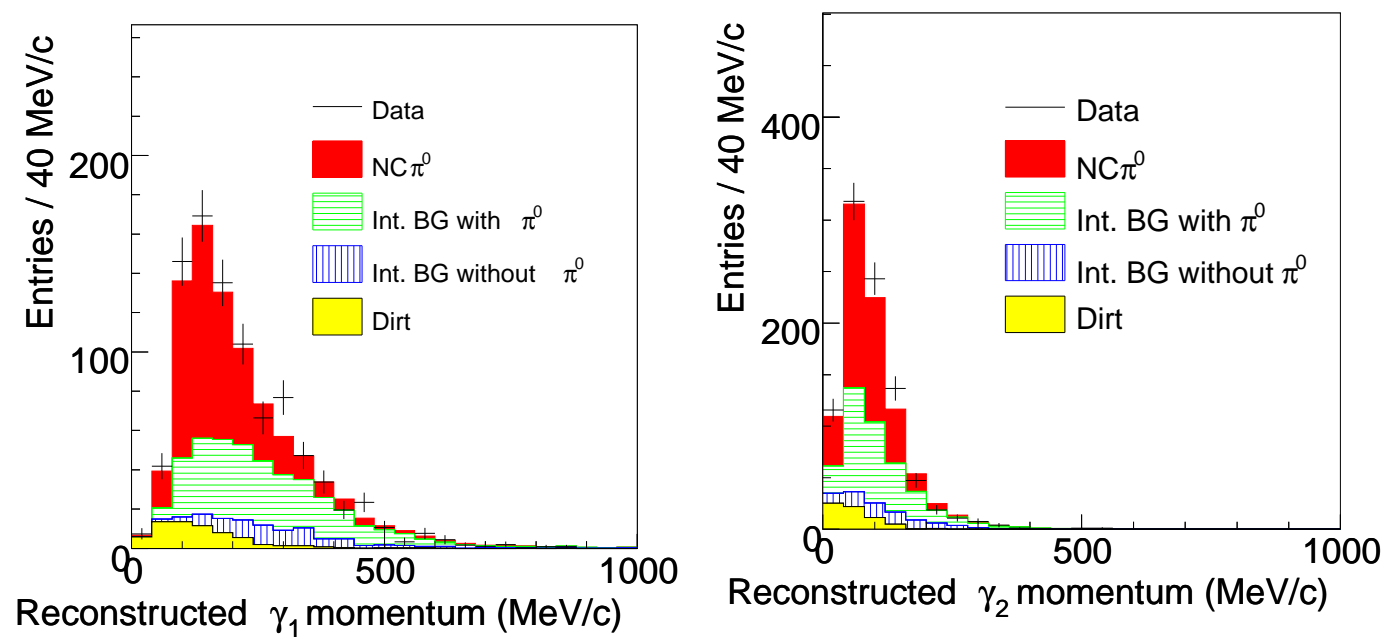

Figure 6.4: $\mathrm{E}_{\gamma 1}^{\mathrm{rec}}$ and $\mathrm{E}_{\gamma 2}^{\mathrm{rec}}$ before the $\pi^{0}$ mass cut $\left(\mathrm{E}_{\gamma 1}^{\mathrm{rec}}>\mathrm{E}_{\gamma 2}^{\mathrm{rec}}\right)$

\subsubsection{Reconstructed $\pi^{0}$ Momentum}

Figure 6.5 shows the reconstructed $\pi^{0}$ momentum after the $\pi^{0}$ mass cut. The average momentum of the selected $\pi^{0} \mathrm{~s}$ and the $\pi^{0}$ momentum resolution are estimated to be 223 $\mathrm{MeV} / c$ and $17 \%$, respectively (Appendix A). The $\mathrm{NC} \pi^{0}$ efficiency as a function of $\pi^{0}$ momentum is shown in Figure 6.6. Using these reconstructed $\pi^{0}$ momentum and the efficiency, the true $\pi^{0}$ momentum information is extracted in Section 6.3.5

$$
\mathrm{P}_{\pi^{0}}^{\mathrm{rec}}=\sqrt{\mathrm{E}_{\gamma 1}^{\mathrm{rec} 2}+\mathrm{E}_{\gamma 2}^{\mathrm{rec} 2}+2 \mathrm{E}_{\gamma 1}^{\mathrm{rec}} \mathrm{E}_{\gamma 2}^{\mathrm{rec}} \cos \theta^{\mathrm{rec}}}
$$

$\mathrm{E}_{\gamma 1}^{\mathrm{rec}}\left(\mathrm{E}_{\gamma 2}^{\mathrm{rec}}\right)$ and $\theta^{\text {rec }}$ are same definition as the formula for the reconstructed $\pi^{0}$ mass. 


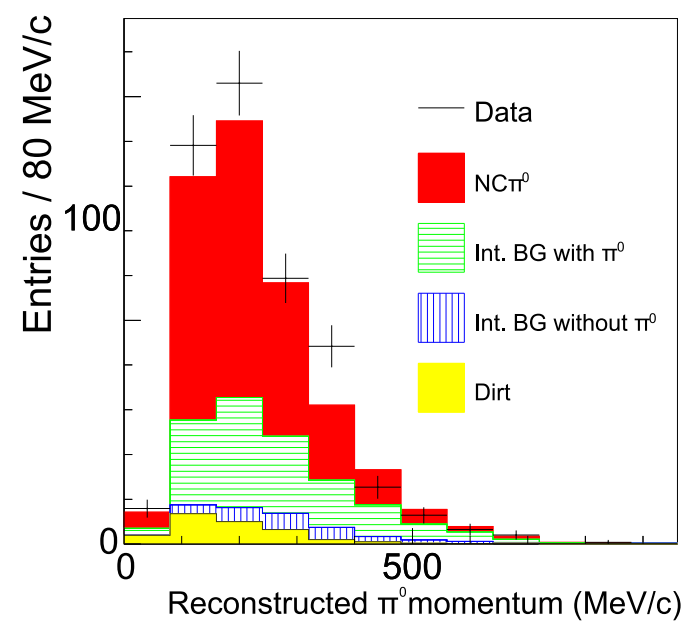

Figure 6.5: Reconstructed $\pi^{0}$ momentum after the $\pi^{0}$ mass cut
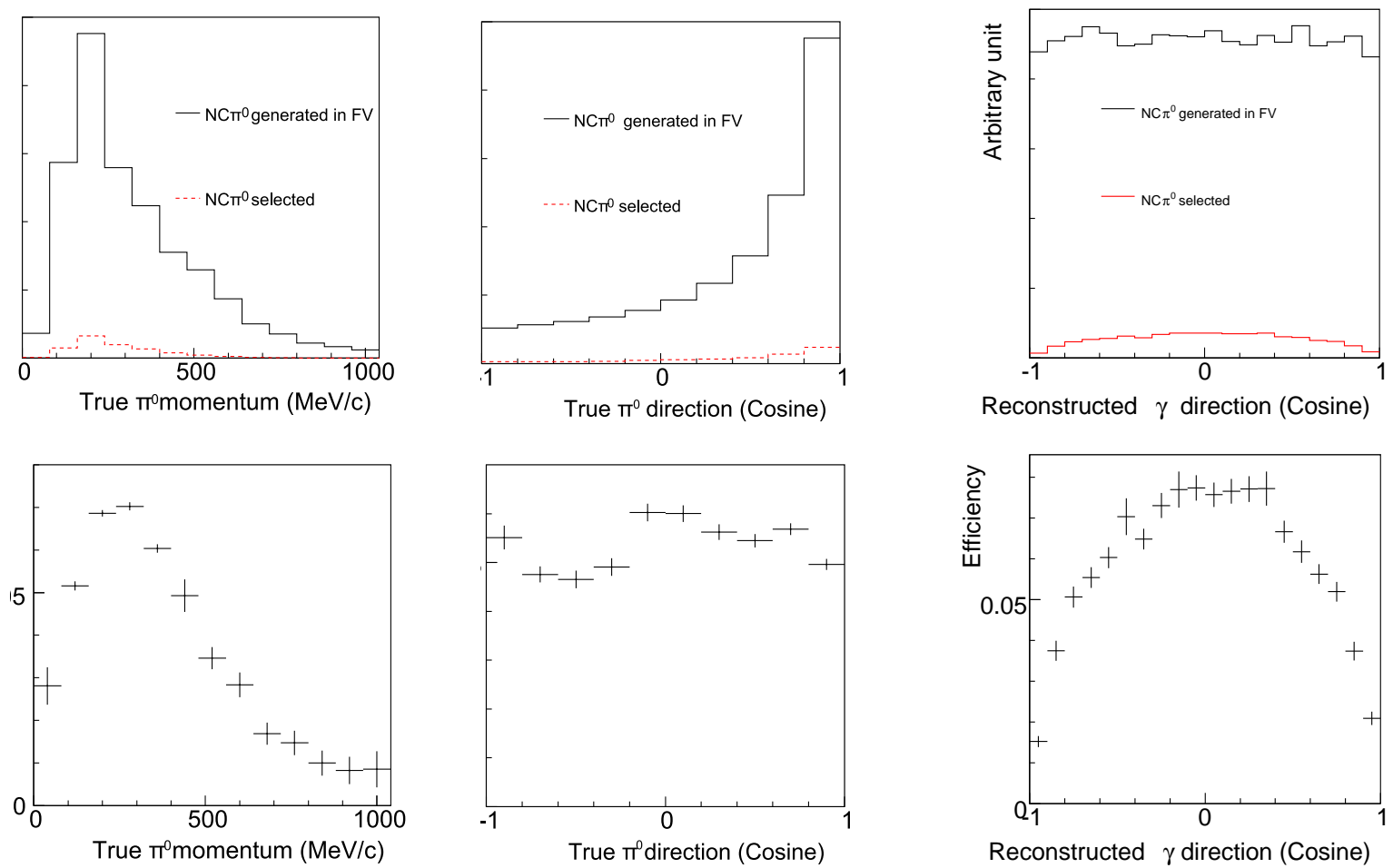

Figure 6.6: The upper three plots show the distributions of the true $\pi^{0}$ momentum and $\pi^{0}$ direction, gamma direction for the generated $\mathrm{NC} \pi^{0}$ events and selected $\mathrm{NC} \pi^{0}$ events. The lower three figures show the efficiency dependence of the the true $\pi^{0}$ momentum and $\pi^{0}$ direction, gamma direction. 


\subsubsection{Reconstructed $\pi^{0}$ Angle}

Figure 6.7 shows the reconstructed $\pi^{0}$ angle $\left(\theta_{\pi^{0}}^{\text {rec }}\right)$ with the respect to neutrino beam direction after the $\pi^{0}$ mass cut.

$$
\cos \theta_{\pi^{0}}^{\mathrm{rec}}=\frac{\mathrm{P}_{\mathrm{z} \pi^{0}}^{\mathrm{rec}}}{\mathrm{P}_{\pi^{0}}^{\mathrm{rec}}}
$$

Here, $\mathrm{P}_{\mathrm{z} \pi^{0}}^{\text {rec }}$ is $\mathrm{z}$ (beam axis) projection of $\mathrm{P}_{\pi^{0}}^{\text {rec }}$. The angular resolution of the $\pi^{0}$ direction is estimated to be 6 degrees in the MC simulation (Appendix A). The $\mathrm{NC} \pi^{0}$ efficiency as a function of $\pi^{0}$ angle is shown in Figure 6.6. These reconstructed $\pi^{0}$ angle and efficiency are used for extracting the true $\pi^{0}$ angle information in Section 6.3.5
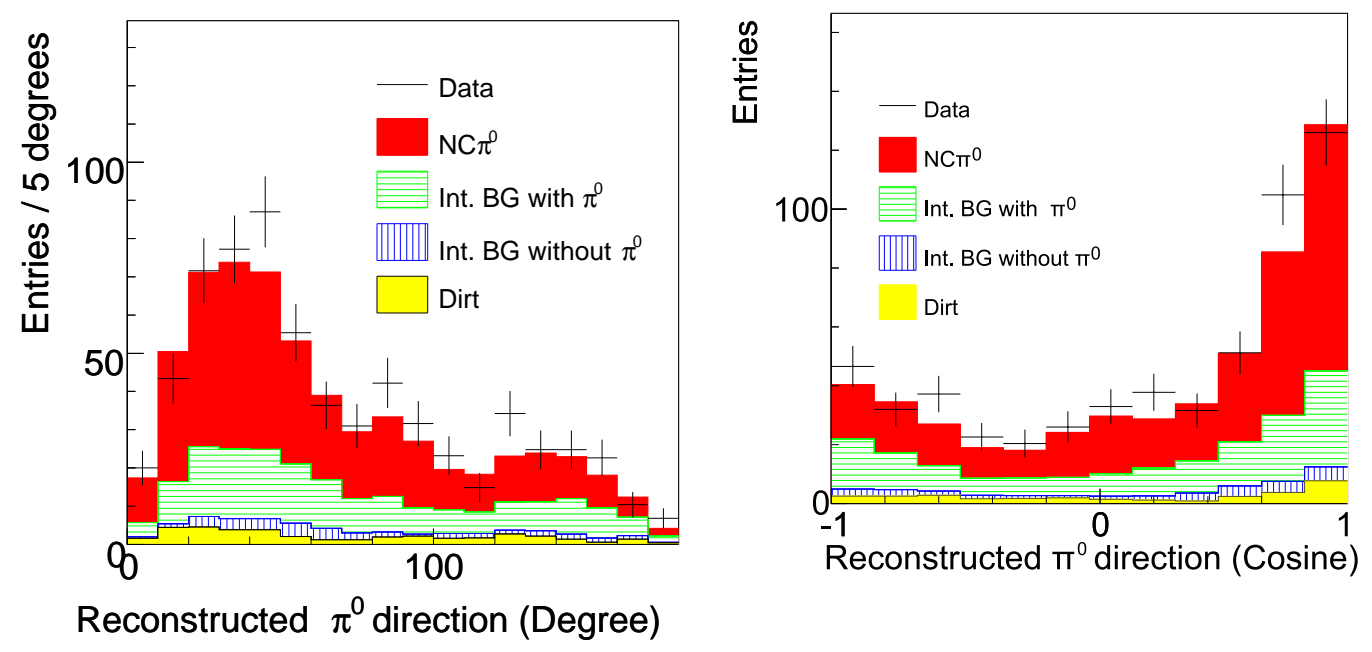

Figure 6.7: Reconstructed $\pi^{0}$ angle after the $\pi^{0} \operatorname{mass} \operatorname{cut}\left(\theta_{\pi^{0}}^{r e c}\right.$ and $\left.\cos \theta_{\pi^{0}}^{\text {rec }}\right)$

\subsubsection{Reconstructed Gamma Angle}

Figure 6.8 shows the reconstructed $\gamma$ angle with respect to the $\pi^{0}$ direction at the $\pi^{0}$ rest frame after the $\pi^{0}$ mass cut.

$$
\begin{array}{r}
\tan \theta_{\gamma}^{*}=\frac{\sin \theta_{\gamma}}{\gamma\left(\cos \theta_{\gamma}-\beta\right)} \\
\beta=\frac{\mathrm{P}_{\pi^{0}}^{\mathrm{rec}}}{\sqrt{\mathrm{P}_{\pi^{0}}^{\mathrm{rec} 2}+\mathrm{M}_{\pi^{0}}^{\mathrm{rec} 2}}} \\
\gamma=\frac{1}{\sqrt{1-\beta^{2}}}
\end{array}
$$

Here, $\theta_{\gamma}^{*}$ is the reconstructed $\gamma$ direction at the $\pi^{0}$ rest frame and $\theta_{\gamma}$ is the reconstructed $\gamma$ angle with respect to the $\pi^{0}$ direction at the laboratory frame. We set $\theta_{\gamma}^{*}=0$ when the reconstructed $\gamma$ direction at the $\pi^{0}$ rest frame is same as the $\pi^{0}$ direction at the laboratory frame. The $\mathrm{NC} \pi^{0}$ efficiency as a function of the reconstructed gamma angle $\left(\cos \theta_{\gamma}^{*}\right)$ is shown in Figure 6.6. Due to the two body decay, the $\cos \theta_{\gamma}^{*}$ distribution is flat in the ideal 
case. But actual $\cos \theta_{\gamma}^{*}$ distribution is not flat around $\cos \theta_{\gamma}^{*}=-1$ and $\cos \theta_{\gamma}^{*}=1$ due to the following reason. If one of $2 \gamma$ has most of the parent $\pi^{0}$ 's momentum, the boosting direction ( $\pi^{0}$ momentum direction) to the $\pi^{0}$ rest frame is nearly parallel to the direction of this gamma ray. In this case, the directions of the $2 \gamma$ at the $\pi^{0}$ rest frame are parallel and anti-parallel to the $\pi^{0}$ direction at the laboratory frame, which means $\cos \theta_{\gamma}^{*} \sim \pm 1$. However, another $\gamma$ with smaller momentum sometimes can not make track due to its momentum below the tracking threshold so that events with such $2 \gamma$ are rejected as events with only one photon candidate (one extended track).
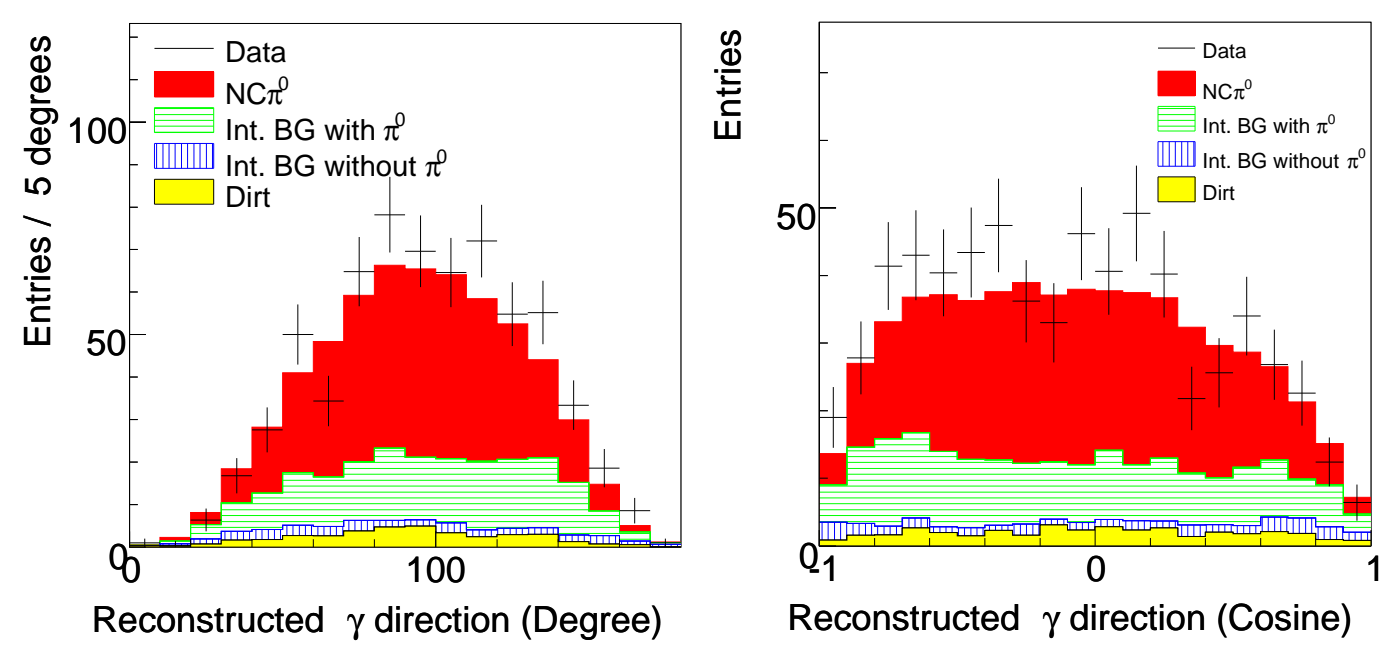

Figure 6.8: $\theta_{\gamma}^{*}$ and $\cos \theta_{\gamma}^{*}$ after the $\pi^{0}$ mass cut (reconstructed $\gamma$ angle with respect to $\pi^{0}$ direction at $\pi^{0}$ rest frame)

\subsubsection{Extraction of True $\pi^{0}$ Momentum and Angular Distribu- tion}

We extract true $\pi^{0}$ momentum and angular distribution. First we perform the background subtraction using the MC expectation. Then, we unfold the reconstructed $\pi^{0}$ momentum and angle to obtain selected true $\pi^{0}$ momentum and angular distribution using Bayesian method [98]. Finally, we perform the efficiency correction.

\section{Background Subtraction}

We subtract the background estimated by the MC simulation from the $\pi^{0}$ momentum and angular distribution. The MC simulation are normalized by the number of the MRDstopped events. Figure 6.9 show the $\pi^{0}$ momentum and angular distribution. To estimate systematic uncertainties for each bin, we generate 1000 expectations within systematic variations and take RMS of expectations as the size of systematic errors. This method is used for all histograms with systematic uncertainties. The detail description is in Appendix C. All systematic sources described in Section 6.2.4 are taken into account. 

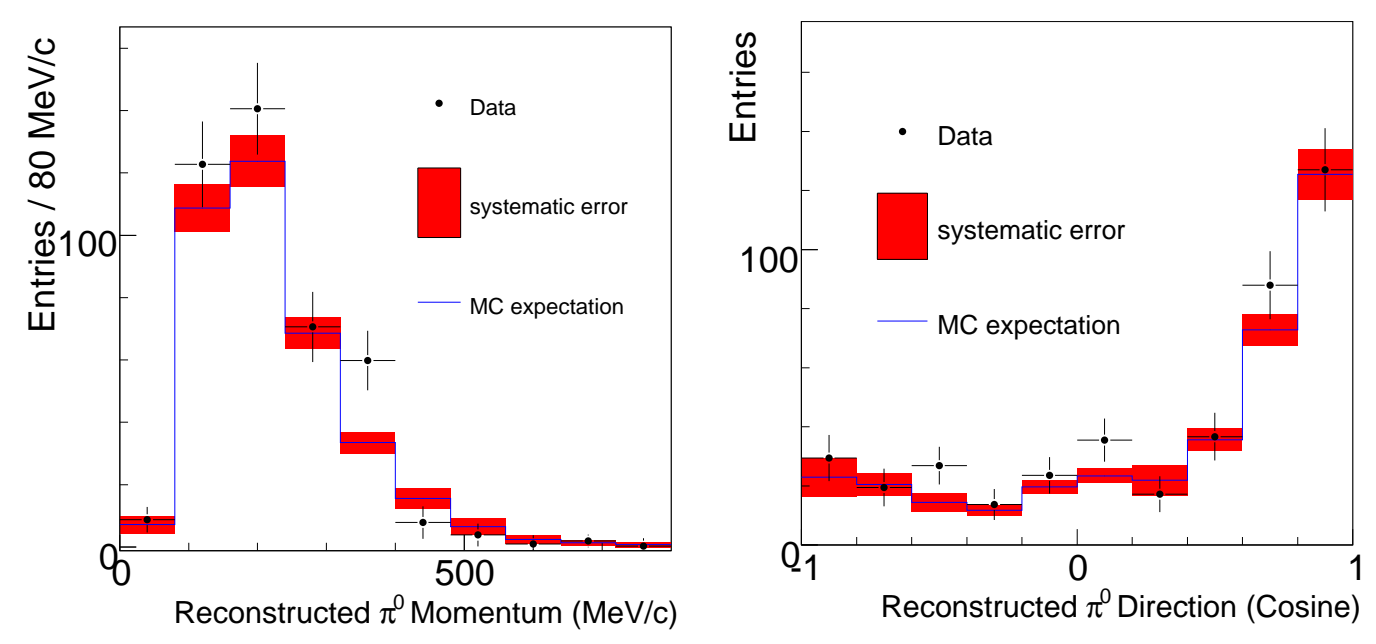

Figure 6.9: The distributions of the reconstructed $\pi^{0}$ momentum and direction after background subtraction. the solid line and the box show the statistic and systematic errors, respectively. The faded line shows the MC expectation

\section{Unfolding}

To convert reconstructed kinematics to true kinematics, we construct $10 \times 10$ unfolding matrices using the MC simulation for $\pi^{0}$ momentum and direction defined as

$$
M_{i, j}=P\left(T_{i} \mid R_{j}\right)
$$

where $P\left(T_{i} \mid R j\right)$ is the conditional probability of the reconstructed kinematics in $j$-th bin to produce the true kinematics in $i$-th bin. By definition, $P\left(T_{i} \mid R j\right)$ should be normalized as

$$
\sum_{i=1}^{10} P\left(T_{i} \mid R j\right)=1
$$

Figure 6.10 shows each contents of the unfolding matrices for the $\pi^{0}$ momentum and direction, respectively. Using the unfolding matrix, we obtain true kinematics distribution as

$$
n_{\text {true }}(i)=\sum_{j=1}^{10} M_{i, j} n_{\text {rec. }}(j)
$$

where $n_{\text {true(rec.) }}(i)$ is the entries in the $i$-th bin of the true (reconstructed) kinematics distributions. The bin widths for the $\pi^{0}$ momentum and angular distribution are $80 \mathrm{MeV} / c$ and 0.2 (Cosine), respectively. We use same binning both for reconstructed and true kinematics distributions. Figure 6.11 shows true $\pi^{0}$ momentum and angular distribution after applying unfolding matrices. The systematic errors of the plots are estimated with the same source described in Section 6.2.4. 

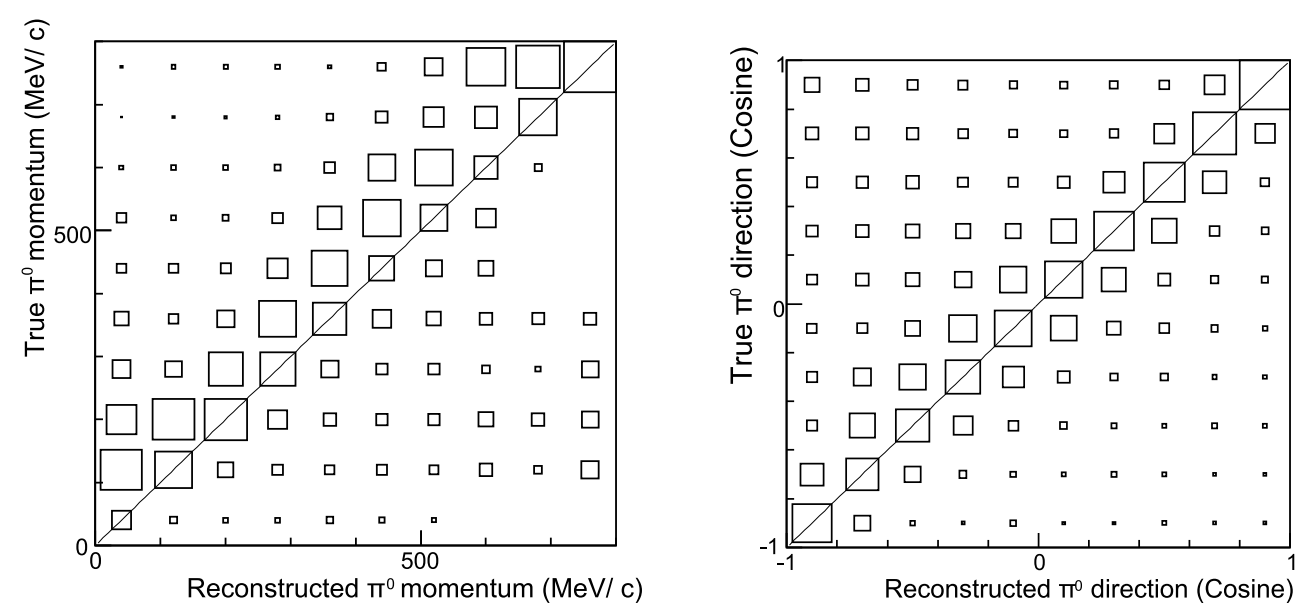

Figure 6.10: The 2D plots between the true $\pi^{0}$ momentum (direction) and the reconstructed one. The solid line shows $y=x$. The size of boxes indicates the content of each unfolding matrix element
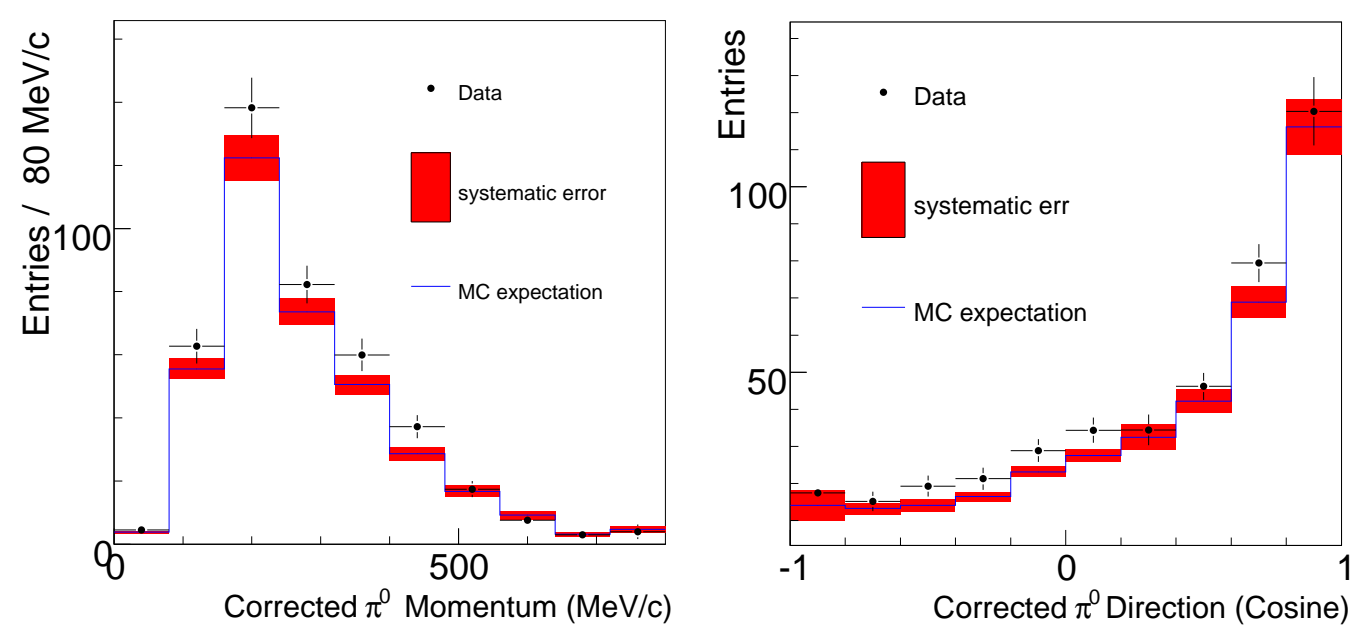

Figure 6.11: The distribution of the true $\pi^{0}$ momentum and direction after the unfolding. the solid line and the box show the statistic and systematic errors, respectively. The faded line shows the MC expectation

\section{Efficiency Correction}

After unfolding, the content of each bin is divided by the detection efficiency shown in Figure 6.6. Figure 6.12 show the distributions after the efficiency correction. The difference of total number of events for both distributions is $11 \%$, which is same as the difference between the measured center value and the MC expectation of the cross section ratio described in Section 6.2.3. 

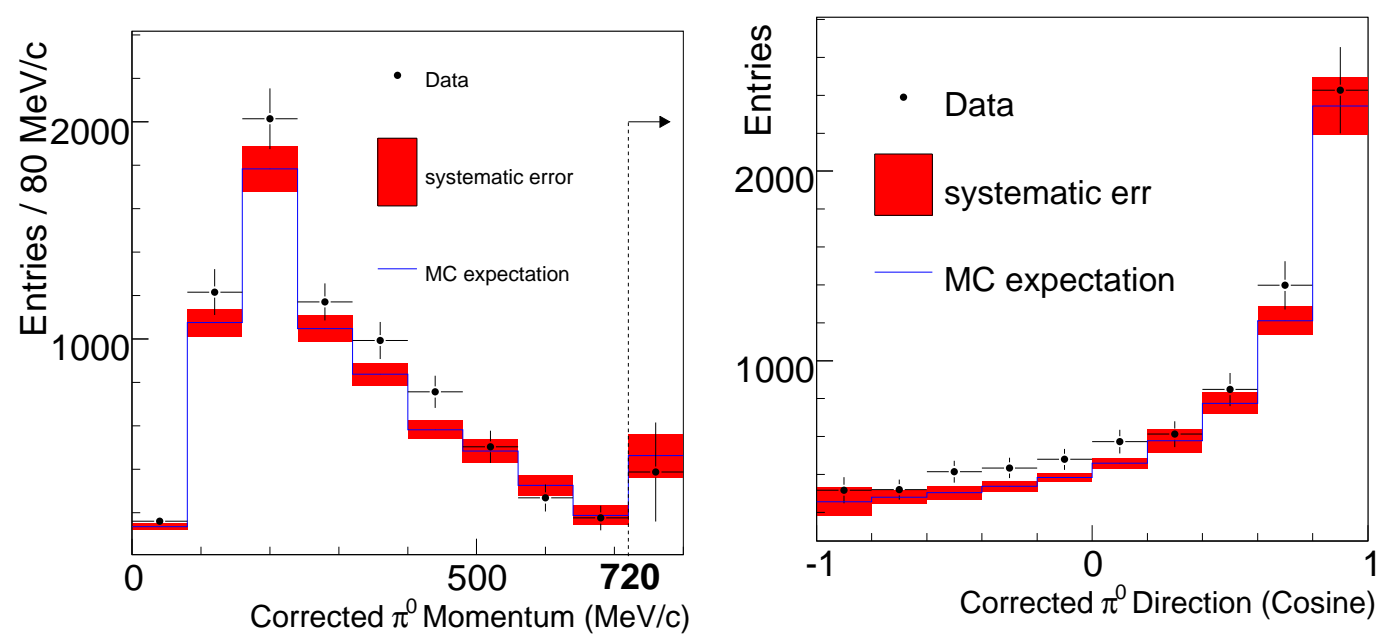

Figure 6.12: The distributions of the $\pi^{0}$ momentum and direction after all corrections described in the text. The solid line and the box show the statistic and systematic errors, respectively. The dashed line shows the Monte Carlo expectation based on the Rein and Sehgal model.

\section{True $\pi^{0}$ Momentum and Angular Distribution}

To compare the shapes of the distributions between the measurement and the expectation, we normalize both distribution by unit as shown in Figure 6.12. The shape of the distributions of the measurement reasonably agree with the $\mathrm{MC}$ expectation. This means that not only the Rein-Sehgal model but also the intra-nuclear interaction models shown in Section 4.2.2 describe our data since approximately $40 \%$ of neutrino-induced pions interact with the nuclear matter.

\subsection{Coherent Pion Production}

In this section, we measure the contribution of coherent pion production in the $\mathrm{NC} \pi^{0}$ events.

\subsubsection{Vertex Activity}

In the NC coherent pion production, there is no recoil nucleon at the final state since the $\pi^{0}$ is produced by neutrino interaction with a whole nucleus. Meanwhile, the nucleon recoil occurs in resonant pion production. To separate the $\mathrm{NC}$ coherent $\pi^{0}$ events from the $\mathrm{NC}$ resonant $\pi^{0}$ events, the recoil protons in the final state is used. The recoil protons are detected by their large energy deposition around the neutrino interaction vertex, so-called vertex activity. We search for the maximum deposited energy in a scintillator strip around the reconstructed vertex, an area of $40 \mathrm{~cm} \times 40 \mathrm{~cm}$ in both view. The reconstructed vertex is defined in Section 5.5.10. Since the part of selected two extended tracks $(2 \gamma$ candidate) is in the search region, we do not count hits associated with original tracks in the selected two extended tracks. The newly added hits in the extended tracks are counted as the vertex activity because the newly added hits are far from original tracks by up to 20 

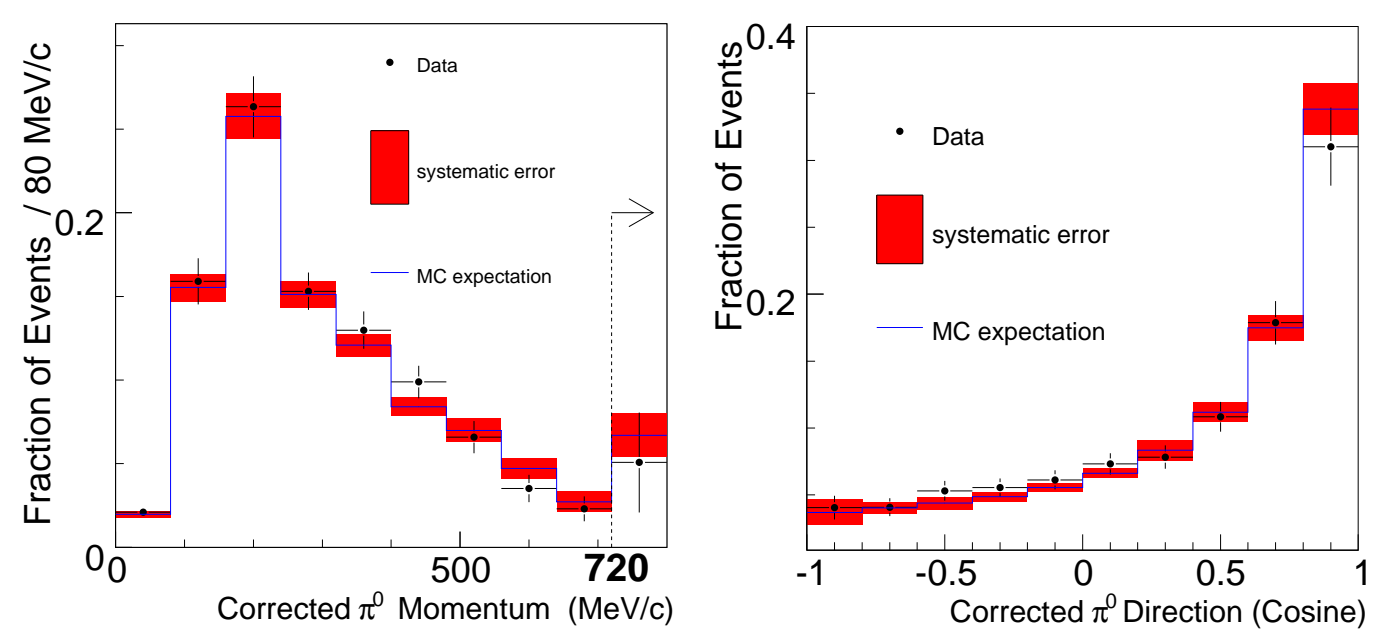

Figure 6.13: The distributions of the $\pi^{0}$ momentum and direction after all corrections described in the text. The solid line and the box show the statistic and systematic errors, respectively. The dashed line shows the Monte Carlo expectation based on the Rein and Sehgal model. Both distributions are normalized by unity.

$\mathrm{cm}$ and could be hits by recoil protons. Figure 6.14 shows the maximum deposited energy distribution after all selection cuts. The most of the coherent $\pi^{0}$ contribution is peaked at zero while the other $\pi^{0}$ production have high energy activity due to recoil protons. Events with energy deposition more than $2 \mathrm{MeV}$ are considered to have the activity at the vertex. Table 6.4 shows the number of events for samples with and without the vertex activity. The number of events in samples with and without the vertex activity in data are 406 and 251 while those in MC are 382 and 227, respectively. Figure 6.15 and 6.16 show the distributions of the reconstructed $\pi^{0}$ momentum and direction with and without the vertex activity. The $\pi^{0}$ events without the vertex activity tends to go forward comparing to that with the vertex activity, which is another feature of coherent pion production.

\begin{tabular}{ccc}
\hline \hline Mode & with vertex activity & without vertex activity \\
\hline NC coherent $\pi^{0}$ & 10 & 81 \\
NC incoherent $\pi^{0}$ & 194 & 83 \\
NC other & 42 & 20 \\
CC with $\pi^{0}$ & 97 & 23 \\
CC without $\pi^{0}$ & 13 & 8 \\
Dirt BG & 26 & 11 \\
\hline MC total & 382 & 227 \\
\hline Data & 406 & 251 \\
\hline \hline
\end{tabular}

Table 6.4: The number of events for both data and MC samples with and without the vertex activity. 


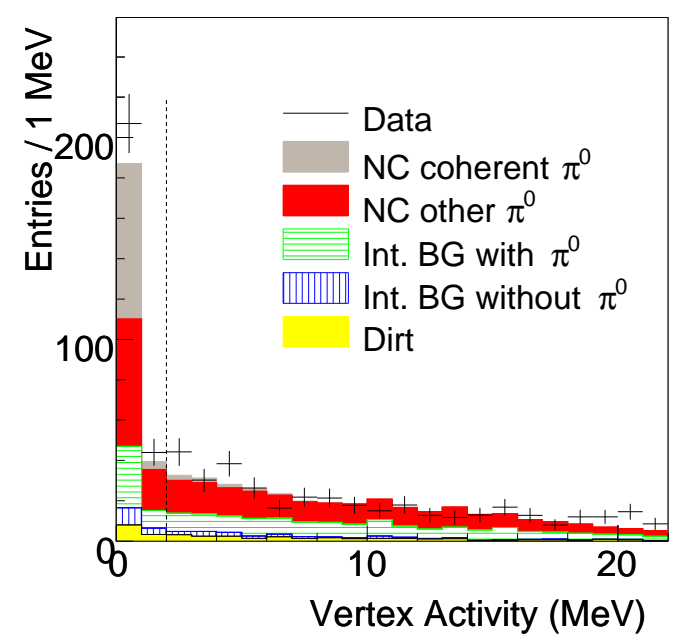

Figure 6.14: The vertex activity after all event selection cuts: The coherent contribution and other $\mathrm{NC} \pi^{0}$ are separately shown in the MC simulation.
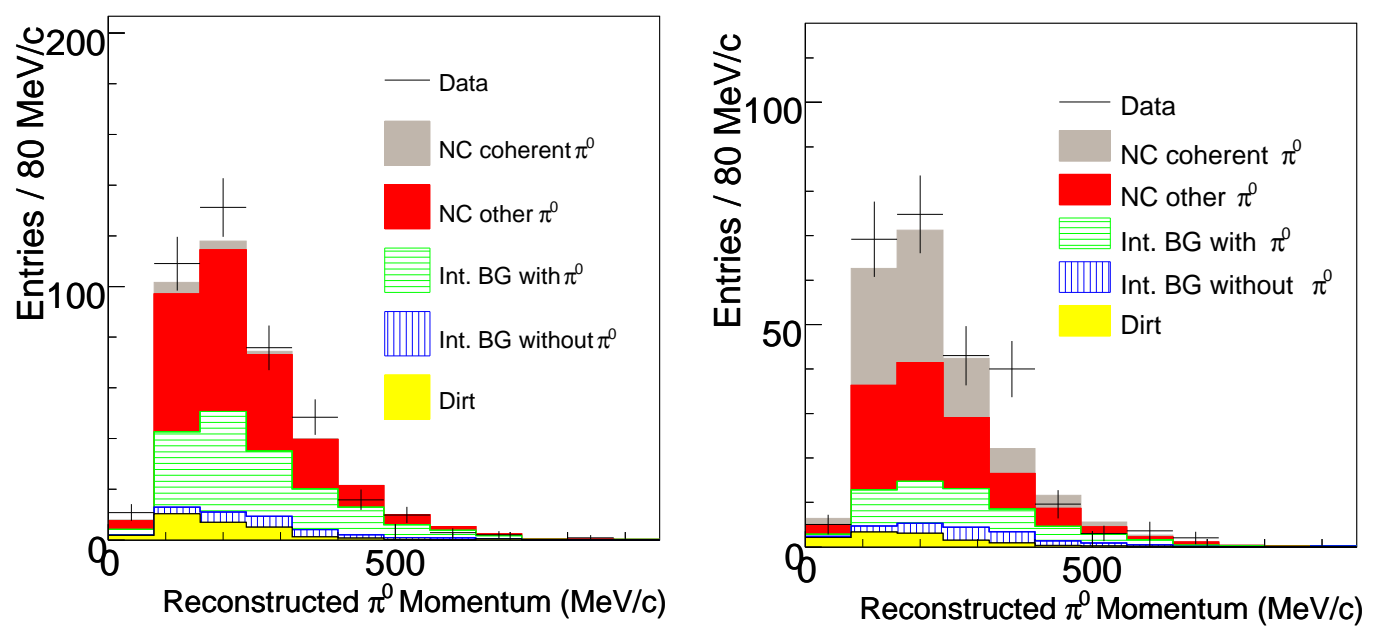

Figure 6.15: Reconstructed $\pi^{0}$ momentum distributions after all selection cuts with the vertex activity (left) and without the vertex activity (right)

\subsubsection{Coherent Pion Extraction}

The coherent and resonant production have very different distributions of the pion angle with respect to the beam direction. This fact can be used to separate the relative contribution of the two production mechanisms.

To extract the fraction of coherent $\pi^{0}$ production, we use the kinematic distribution of $E_{\pi^{0}}^{\mathrm{rec}}\left(1-\cos \theta^{\mathrm{rec}}\right)$ with and without vertex activity shown in Figure 6.17 , where $E_{\pi^{0}}^{\mathrm{rec}}$ is the reconstructed $\pi^{0}$ energy calculated as $E_{\gamma 1}^{\mathrm{rec}}+E_{\gamma 2}^{\mathrm{rec}}$. As we mention in Section 1.3.2, the coherent pion production satisfies

$$
\frac{1}{|t|}>R
$$



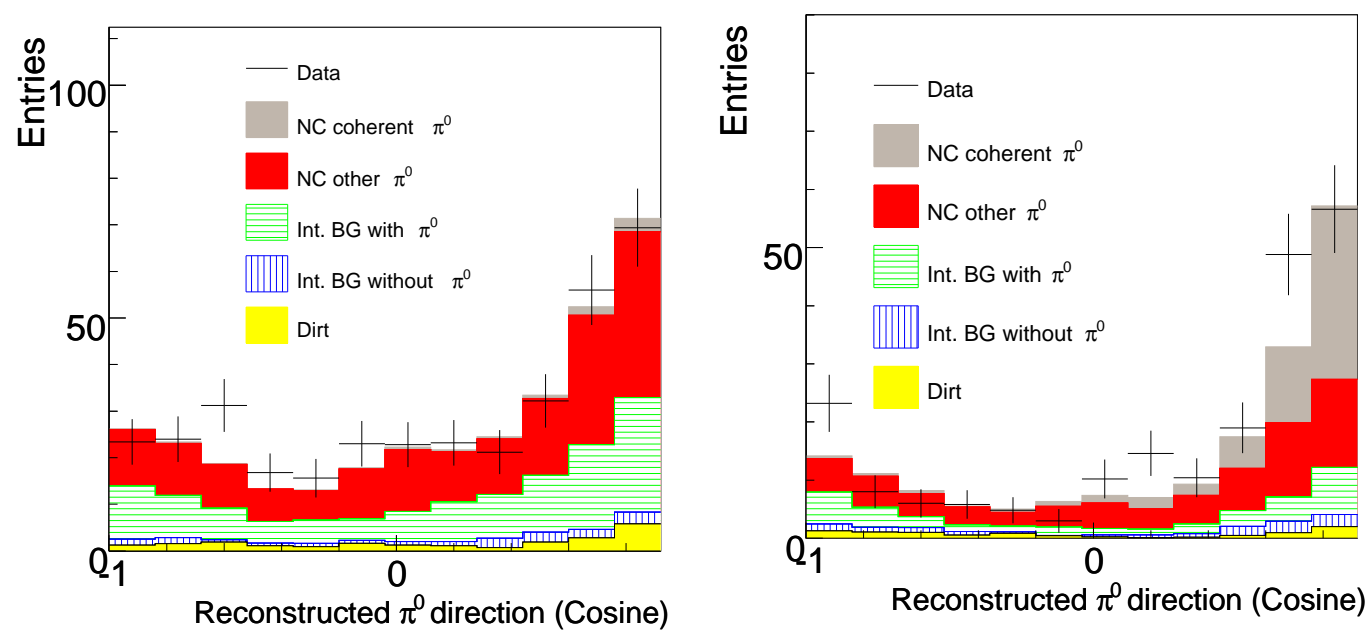

Figure 6.16: Reconstructed $\pi^{0}$ direction distributions after all selection cuts with the vertex activity (left) and without the vertex activity (right)

where $t$ and $R$ are the four-momentum transfer to the target nucleus from the neutrino and the radius of target nucleus, respectively. This means that the cross section decrease rapidly when $1 /|t|$ become smaller than $R$. Then, the condition expressed as

$$
\frac{1}{t^{2}}>R^{2}
$$

is also satisfied for the coherent pion production. From Equation 6.14, one can deduce

$$
E_{\pi^{0}}\left(1-\cos \theta_{\pi^{0}}\right)<\frac{1}{R}
$$

as described in [99]. Here, the $E_{\pi^{0}}$ and $\theta_{\pi^{0}}$ are the energy and direction of the emitted $\pi^{0}$, respectively. This is why the $E_{\pi^{0}}^{\mathrm{rec}}\left(1-\cos \theta^{\mathrm{rec}}\right)$ is a good valuable to separate the coherent $\pi^{0}$ contribution from the resonant $\pi^{0}$ production as well as $t$. We fit two $E_{\pi^{0}}^{\mathrm{rec}}\left(1-\cos \theta^{\mathrm{rec}}\right)$ distributions with and without the vertex activity by using three templates made by dividing the final MC sample into $\mathrm{NC}$ coherent $\pi^{0}$, NC resonant $\pi^{0}$ and background samples. Two parameters, $\mathrm{R}_{\text {coh }}$ and $\mathrm{R}_{\text {res }}$ scale the $\mathrm{NC}$ coherent $\pi^{0}$ and $\mathrm{NC}$ resonant $\pi^{0}$ templates independently. The background sample is fixed to the value of the $\mathrm{MC}$ prediction although the systematic errors on the background prediction are taken into account. The expected number of events in the $i$-th bin in the $E_{\pi^{0}}^{\mathrm{rec}}\left(1-\cos \theta^{\mathrm{rec}}\right)$ distribution is expressed as:

$$
N_{i}^{\text {exp }}=\mathrm{R}_{\text {coh }} \times N_{i}^{\text {coh }}+\mathrm{R}_{\text {res }} \times N_{i}^{\text {res }}+N_{i}^{\text {BG }}
$$

The fit minimizes the following $\chi^{2}$ :

$$
\chi^{2}=-2 \ln \frac{f\left(N^{\text {obs }} ; N^{\exp }\right)}{f\left(N^{\text {obs }} ; N^{\text {obs }}\right)},
$$

where $N^{\text {obs(exp) }}$ represents the observed (expected) number of events in all bins $\left(N_{1}^{\text {obs }(\exp )}, N_{2}^{\text {obs(exp) }}, \ldots, N_{N}^{\text {obs(exp) }}\right)$ and $f\left(N^{\text {obs }} ; N^{\exp }\right)$ is the Poisson likelihood to find $N^{\text {obs }}$ 

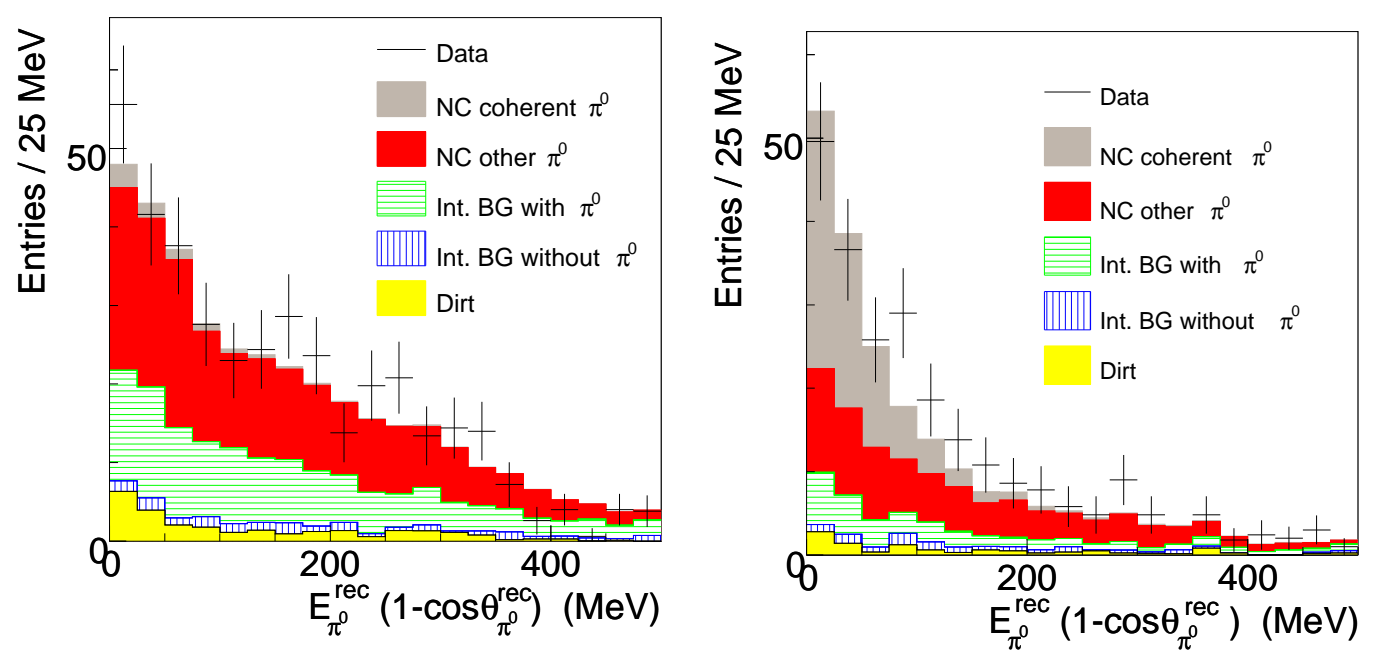

Figure 6.17: The $E_{\pi^{0}}^{\mathrm{rec}}\left(1-\cos \theta^{\text {rec }}\right)$ distributions before fitting with (left) and without (right) vertex activity

events when $N^{\exp }$ events are expected. When the systematic errors for each bin and their correlation expressed with covariance matrix $V_{j k}(j, k=1,2, \ldots, N(=39))$ are given, the likelihood is expressed as

$$
\begin{aligned}
& f\left(N^{\mathrm{obs}} ; N^{\exp } ; V\right)=A \int\left[\left[\prod_{i=1}^{N} d x_{i} \frac{x_{i}^{N_{i}^{\mathrm{obs}}} e^{-x_{i}}}{N_{i}^{\mathrm{obs} !}}\right]\right. \\
& \left.\times \exp \left[-\frac{1}{2} \sum_{j=1}^{N} \sum_{k=1}^{N}\left(x_{j}-N_{j}^{\exp }\right) V_{j k}^{-1}\left(x_{k}-N_{k}^{\exp }\right)\right]\right]
\end{aligned}
$$

where $A$ is the normalizing constant. To calculate this integral, we generate $1000 \mathrm{MC}$ expectations with random variations drawn within the systematic uncertainties for each bin. All systematic sources described in Section 6.2.4 are taken into account. Using $x_{i, m}$ for the $m$-th expectation in the $i$-th bin, the likelihood is expressed as :

$$
f\left(N^{\mathrm{obs}} ; N^{\exp } ; V\right)=\frac{1}{M} \sum_{m} \prod_{i} \frac{x_{i, m}^{N_{i}^{\mathrm{obs}}} e^{-x_{i, m}}}{N_{i}^{\mathrm{obs}}},
$$

where $M$ is the total number of random samples (1000). The result of the fit is:

$$
\begin{aligned}
\mathrm{R}_{\mathrm{coh}} & =0.97 \pm 0.19 \\
\mathrm{R}_{\mathrm{res}} & =1.25 \pm 0.13
\end{aligned}
$$

The $E_{\pi^{0}}^{\mathrm{rec}}\left(1-\cos \theta^{\mathrm{rec}}\right)$ distribution after the fitting is shown in Figure 6.19. The $\chi^{2}$ per degree of freedom (DOF), before the fit is $31.5 / 39=0.81$, and it is $26.5 / 37=0.71$ after the fit. Figure 6.18 shows three contours corresponding to one, two and three standard deviations from the best fit point. The statistic error and all systematic errors are included in the errors of $R_{\text {coh }}$ and $R_{\text {res }}$. Without the systematic errors, we obtain $0.98 \pm 0.18$ (stat.) 


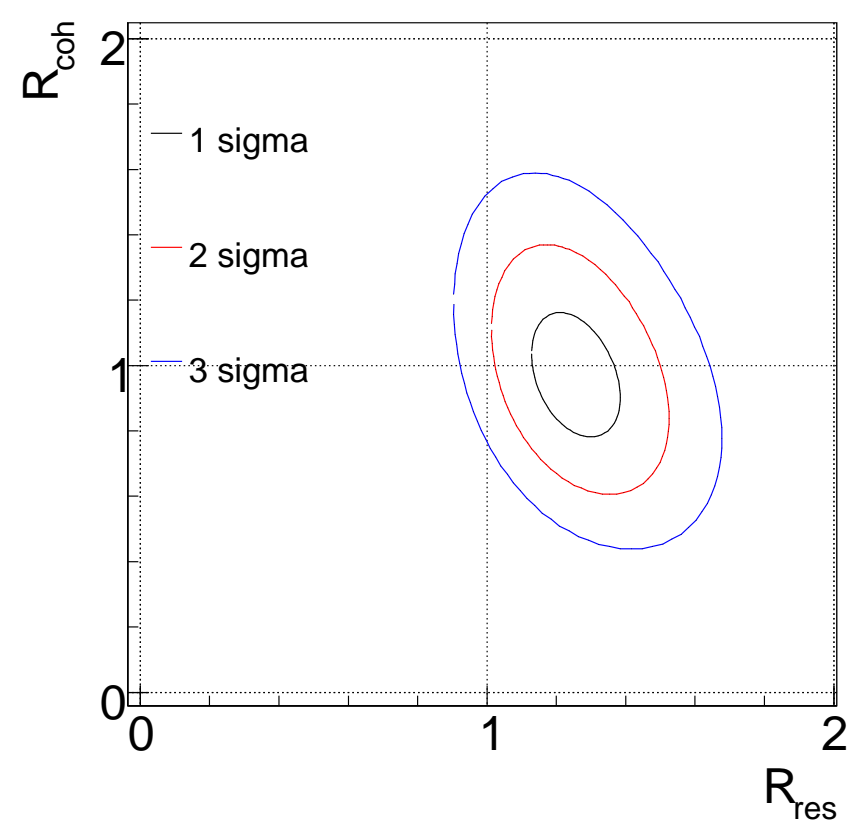

Figure 6.18: The contours corresponding to one, two and three standard deviations from the best fit point

and $1.19 \pm 0.10$ (stat.) for $R_{\text {coh }}$ and $R_{\text {res }}$, respectively. Hence, the uncertainty of the measurement are dominated by the statistic uncertainty. The dominant systematic source is the detector response. Figure 6.20 and 6.21 show the distributions of the reconstruction $\pi^{0}$ momentum and direction with or without the vertex activity after fitting.
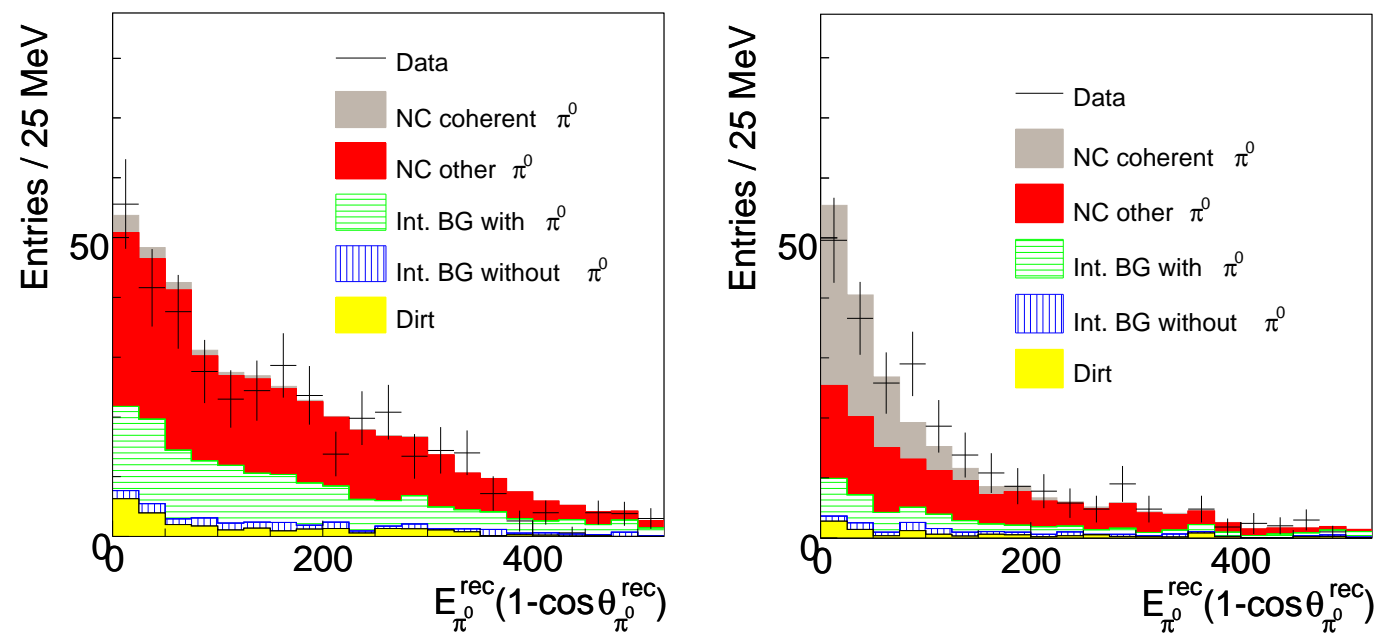

Figure 6.19: The $E_{\pi^{0}}^{\mathrm{rec}}\left(1-\cos \theta^{\text {rec }}\right)$ distributions after fitting with (left) and without (right) vertex activity

The ratio of the NC coherent $\pi^{0}$ production to the total CC cross sections from the MC prediction based on the Rein and Sehgal model is $1.21 \times 10^{-2}$. Hence, the cross 

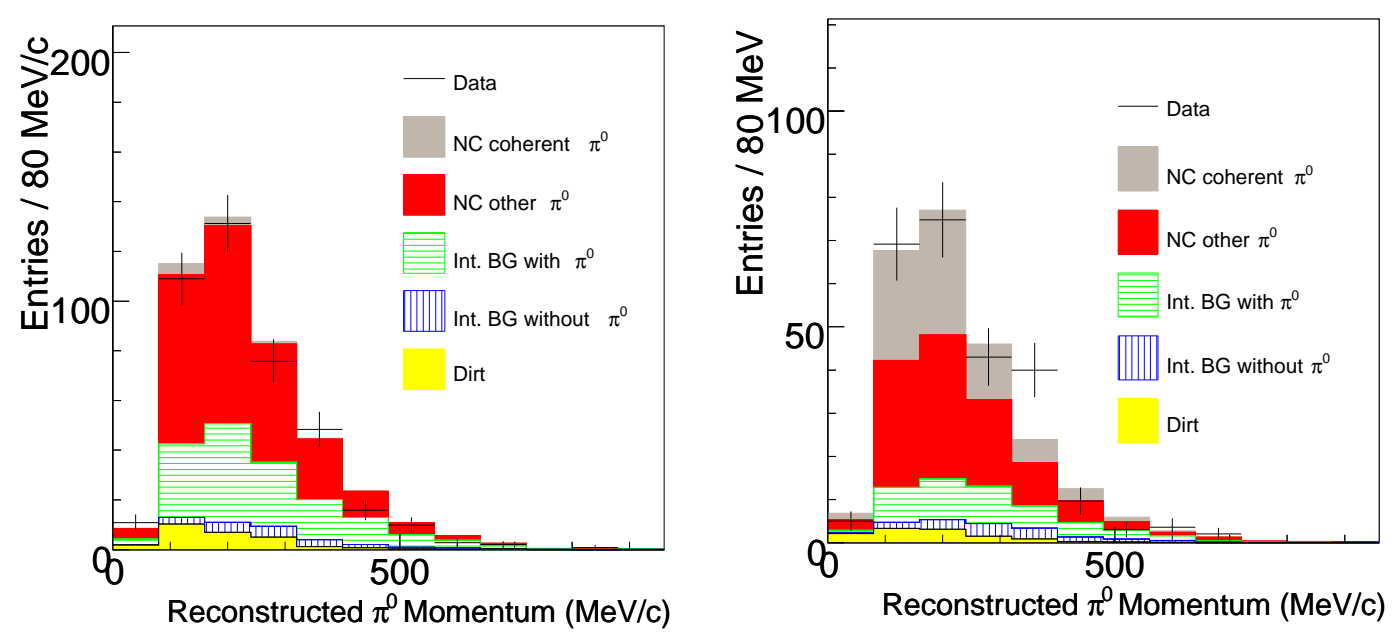

Figure 6.20: Reconstructed $\pi^{0}$ momentum distributions after fitting with the vertex activity (left) and without vertex activity (right)
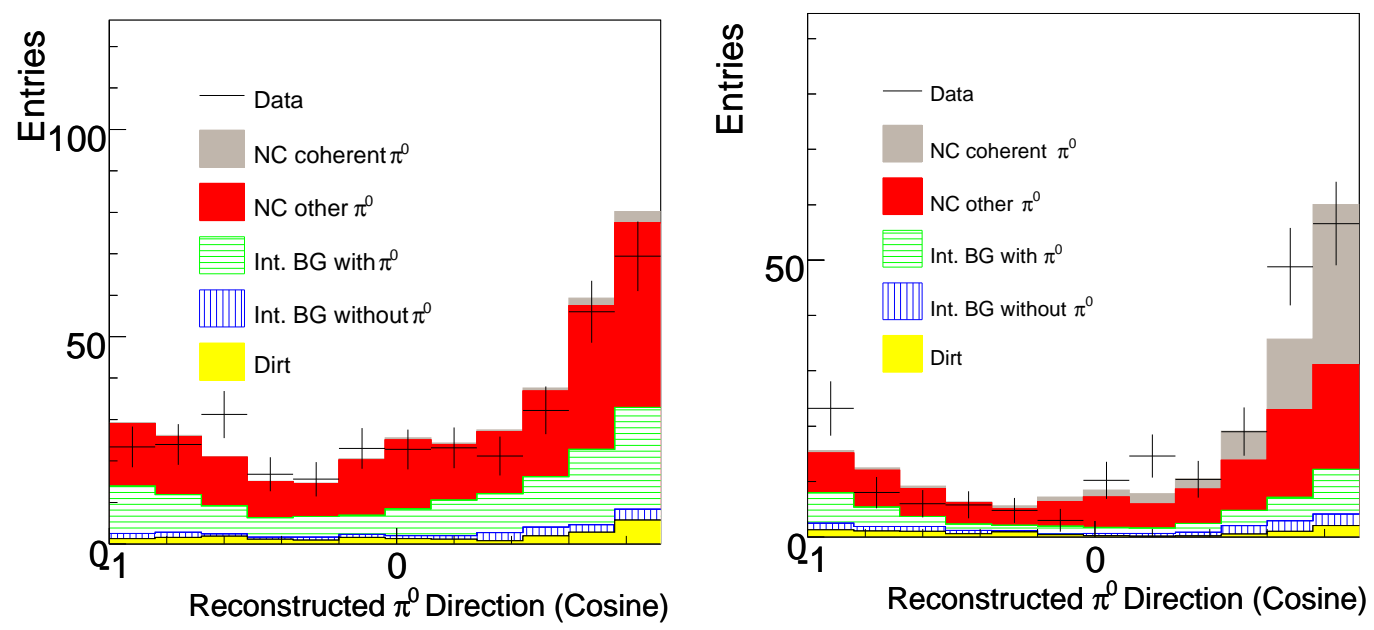

Figure 6.21: Reconstructed $\pi^{0}$ direction distributions after fitting with the vertex activity (left) and without vertex activity (right)

section ratios are measured to be:

$$
\begin{aligned}
\frac{\sigma\left(\mathrm{NCcoh} \pi^{0}\right)}{\sigma(\mathrm{CC})} & =\mathrm{R}_{\mathrm{coh}} \times \frac{\sigma\left(\mathrm{NCcoh} \pi^{0}\right)_{M C}}{\sigma(\mathrm{CC})_{M C}} \\
& =\mathrm{R}_{\mathrm{coh}} \times 1.21 \times 10^{-2} \\
& =(1.17 \pm 0.23) \times 10^{-2}
\end{aligned}
$$

where $R_{\text {coh }}$ is $0.97 \pm 0.19$. The mean neutrino energy for $\mathrm{NC}$ coherent $\pi^{0}$ events in the sample is estimated to be $1.0 \mathrm{GeV}$. This result is 5.8 standard deviations above the no coherent production assumption. The measured cross section is also consistent with the MC prediction based on the Rein and Sehgal model [32]. The result is an evidence of 
non-zero coherent pion production via neutral current interaction at the mean neutrino energy of $1.0 \mathrm{GeV}$.

\subsection{Summary}

The ratio of the $\mathrm{NC} \pi^{0}$ production to total $\mathrm{CC}$ cross sections is measured to be $[7.7 \pm$ 0.5 (stat.) \pm 0.5 (sys.) $] \times 10^{-2}$ at mean neutrino energy $1.1 \mathrm{GeV}$. The MC prediction based on the Rein and Sehgal model [18] is $6.8 \times 10^{-2}$. We achieve less than $10 \%$ uncertainty which is required for the $\nu_{\mu} \rightarrow \nu_{e}$ oscillation measurement down to the sensitivity of $\sin ^{2} 2 \theta_{13} \sim 10^{-2}$.

The measured shapes of the $\pi^{0}$ momentum and angular distributions, as shown in Figure 6.12, agree with the MC predictions within uncertainties. This means that the intra-nuclear interaction models implemented in NEUT well describe the kinematics of $\mathrm{NC} \pi^{0}$ production within the measured error $(\sim 10 \%)$.

The cross section ratio of $\mathrm{NC}$ coherent $\pi^{0}$ production to the total $\mathrm{CC}$ interaction is measured to be $(1.17 \pm 0.23) \times 10^{-2}$ based on the Rein and Sehgal model [32], while the MC prediction is $1.21 \times 10^{-2}$. This result is an evidence of non-zero coherent pion production via neutral current interaction at the mean neutrino energy of $1.0 \mathrm{GeV}$.

\subsection{Discussion}

We achieve three goals mentioned at the beginning: the measurement of the $\mathrm{NC} \pi^{0}$ cross section ratio, the $\pi^{0}$ kinematics distribution and the coherent $\pi^{0}$ contribution.

In this session, first, we discuss the impact of our results on the neutrino physics and the future prospects for each topic. Then, we discuss the possibility of increasing the $\mathrm{NC} \pi^{0}$ detection efficiency in future experiments.

\subsubsection{Impact of our Results on Neutrino Physics and Future Prospects}

\section{$\mathrm{NC} \pi^{0}$ Cross Section}

We achieve less than $10 \%$ uncertainty for the $\mathrm{NC} \pi^{0}$ cross section ratio $\left(\sigma\left(N C \pi^{0}\right) / \sigma(C C)\right)$ so that we can predict the $\mathrm{NC} \pi^{0}$ background events precise enough for the $\nu_{\mu} \rightarrow \nu_{e}$ oscillation search at the sensitivity of $\sin ^{2} 2 \theta_{13}$ down to $10^{-2}$.

However, this is just requirement for the T2K phase I. If $\nu_{\mu} \rightarrow \nu_{e}$ is not observed in $\sin ^{2} 2 \theta_{13} \geq 10^{-2}$, a further $\nu_{\mu} \rightarrow \nu_{e}$ search down to $\sin ^{2} 2 \theta_{13} \sim 10^{-3}$ may be conducted in the T2K phase II with the upgraded accelerator and Hyper-Kamiokande [14]. In this case, the $5 \%$ uncertainty for the $\mathrm{NC} \pi^{0}$ cross section is required to obtain the sensitivity of $\sin ^{2} 2 \theta_{13} \sim 10^{-3}$ [14]. For more precise measurements, at least, the statistical uncertainty should be decreased by increasing the detection efficiency as described in Section 6.6.2

After we find the $\nu_{\mu} \rightarrow \nu_{e}$ mode, we will search for the CP violation (CP phase $\delta$, as shown in Equation 1.2). To extract the CP phase, we compare the probability of $\bar{\nu}_{\mu} \rightarrow \bar{\nu}_{e}$ transition to that of $\nu_{\mu} \rightarrow \nu_{e}$ transition. Therefore, we have to perform the measurement of $\bar{\nu}_{\mu} \rightarrow \bar{\nu}_{e}$. This requires that the $\mathrm{NC} \pi^{0}$ production by antineutrinos should be measured with the similar uncertainty to $\mathrm{NC} \pi^{0}$ by neutrinos. We also took antineutrino data in 
the SciBooNE experiment. The analysis described in this chapter could be applied to the antineutrino data except that we need subtract the $30 \%$ contamination of neutrinos.

\section{$\pi^{0}$ Kinematics}

Validation of the nuclear model is important because we have to rely on the nuclear model to predict the $\mathrm{NC} \pi^{0}$ cross section with different nuclear targets. For example, we need to predict the $\mathrm{NC} \pi^{0}$ cross section on water for $\mathrm{T} 2 \mathrm{~K}$, which is using a water Cherenkov detector as the far detector.

As described in Section 6.3.5, the good data/MC agreement of the $\pi^{0}$ momentum and angular distributions confirms that the intra-nuclear interaction models implemented in NEUT well describe the kinematics of $\mathrm{NC} \pi^{0}$ production within the measured error $(\sim 10$ $\%$ ). Hence we have a confidence in the nuclear model in NEUT.

However, the nuclear model in NEUT will be not enough to predict the more precise cross section in future. In this case, we need direct measurements with given nuclear targets. For T2K, the measurement with a water target at the neutrino energy below 1 $\mathrm{GeV}$ is desired and plan. To obtain the cross section with various nuclear targets (carbon, iron and lead), the MINER $\nu \mathrm{A}$ experiment is now under construction [100].

\section{Coherent Pion Production}

Although our measurement of the cross section of $\mathrm{NC}$ coherent $\pi^{0}$ production is consistent with the prediction by the Rein and Sehgal model, there are still unresolved questions.

As we describe in Section 4.2.1, the model predicts the relation between the neutral and charged current coherent pion production cross sections as $\sigma(\mathrm{CC})=2 \times \sigma(\mathrm{NC})$. However, SciBooNE [49] reports no observation of CC coherent pion at the neutrino energy range of 1-2 GeV. Hence, the Rein and Sehgal model does not reproduce the SciBooNE data in this sense. In addition, the MiniBooNE also observed NC coherent pion production, but which is $65 \%$ of the Rein and Sehgal prediction, at mean neutrino energy of $1.2 \mathrm{GeV}$ [29]. So far our NC coherent $\pi^{0}$ measurement is also consistent with the MiniBooNE result within our statistical error. We need to increase the statistics to compare our result with MiniBooNE. This may be possible in future experiments as described in Section 6.6.2.

From these facts, the study of coherent pion production is still on going in SciBooNE. For the $\mathrm{CC}$ coherent pion production, we found the data excess comparing to the zero coherent pion prediction in the forward pion sample $\left(\theta_{\pi}<35^{\circ}\right)$ both for neutrino and antineutrino data $[101,102]$. The short summary of these recent study of the CC coherent pion is described in Appendix D. Also for the $\mathrm{NC}$ coherent $\pi^{0}$ production, the detail study of the kinematics of the emitted $\pi^{0}$ is desired. This is also possible by increasing the statistics as described in Section 6.6.2.

\subsubsection{Increasing the Detection Efficiency}

As described above, more precise measurements will be desired in future. In this thesis, for the coherent $\pi^{0}$ fraction, the statistic uncertainty is dominant. For the cross section ratio $\left(\sigma\left(N C \pi^{0}\right) / \sigma(C C)\right)$ measurement, the statistic uncertainty is equal to the systematic uncertainty. Hence, increasing the statistics significantly reduces the uncertainties of our measurements. 
However, as described in Section 5.5.12, the detection efficiency of $\mathrm{NC} \pi^{0}$ is $5.3 \%$, which is small. In this section, we discuss the possibilities of increasing the detection efficiency to reduce the statistical uncertainties. The main reason of the low detection efficiency is small gamma conversion probability of SciBar. The second reason is mis-identification of gamma rays.

\section{Gamma Conversion Probability}

The main reason of the small detection efficiency is the small probability of gamma conversion in SciBar. Since the length of SciBar in the beam direction is four radiation lengths, a significant fraction of gamma rays escape from SciBar without conversion. In $30 \%$ of events with a $\pi^{0}$ emitted in the SciBar fiducial volume, both gamma rays convert in SciBar; in 38\%, only one gamma ray converts in SciBar; in 32\%, neither gamma ray converts in SciBar. Since we aim to reconstruct two gamma rays to identify the $\mathrm{NC} \pi^{0}$ events, the maximum detection efficiency attainable is $30 \%$. Figure 6.22 and 6.23 show the conversion probability of two gamma rays from $\pi^{0}$ as a function of the $\pi^{0}$ kinematics (momentum and direction) and vertices. The probability goes down for the $\pi^{0}$ produced around the edge of SciBar as expected while much less dependence of the $\pi^{0}$ kinematics exists. To increase the gamma conversion probability, we need a detector with longer radiation length. To obtain the longer radiation length, the $\mathrm{T} 2 \mathrm{~K}$ experiment develop a detector optimized for the $\pi^{0}$ detection called "P0D" as one of the near detectors. The basic idea of P0D is inserting lead converters between scintillator planes.
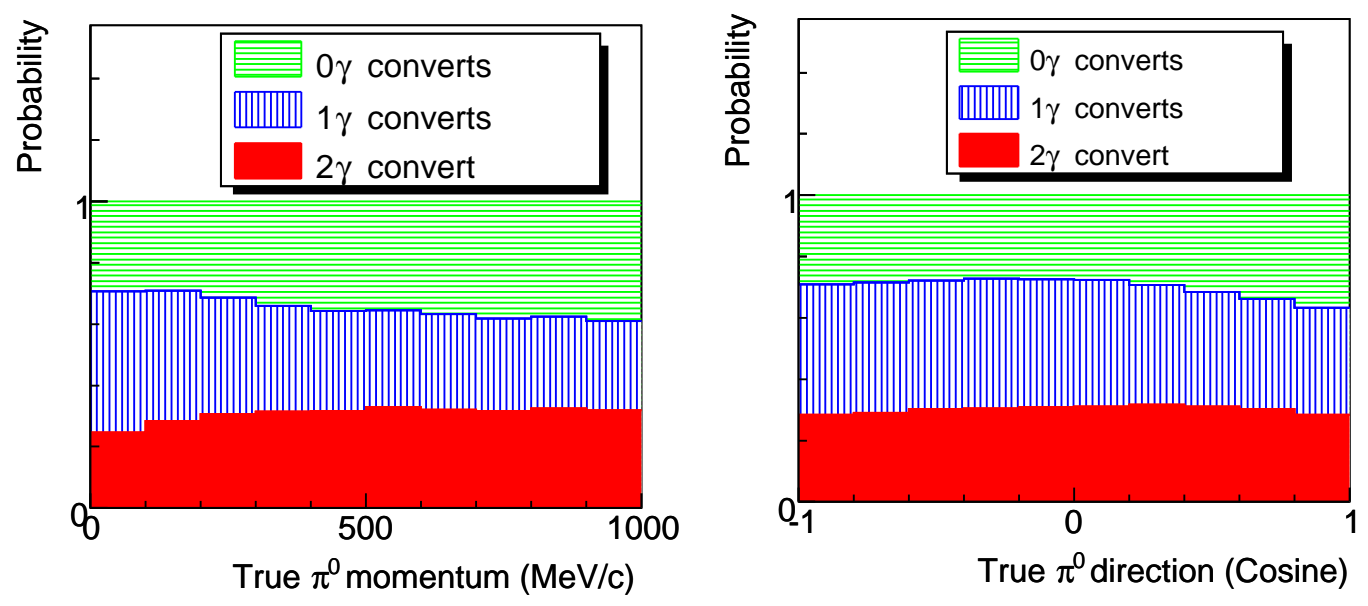

Figure 6.22: The conversion probability of two gamma rays from $\pi^{0}$ as a function of $\pi^{0}$ momentum (left plot) and direction (right plot). The contribution from both gamma rays conversion, one gamma ray conversion and no gamma ray conversion, are shown separately.

\section{Mis-identification of Gamma Ray}

To select gamma rays, we reject the proton and muon candidate tracks. For example, the requirement of at least two extended tracks (Section 5.5.9) rejects about $140 \mathrm{NC} \pi^{0}$ signal events by identifying a gamma ray as a proton-like tracks. There are possibilities 

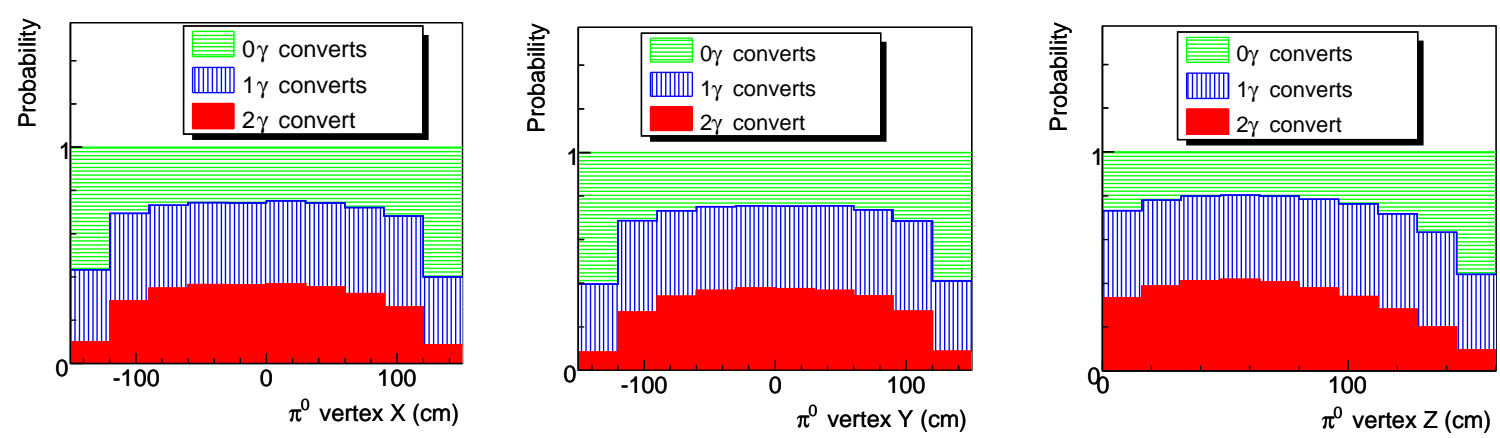

Figure 6.23: The conversion probability of two gamma rays from $\pi^{0}$ as a function of $\pi^{0}$ vertex for each dimension $(x, y, z$ from the left).

to distinguish gamma rays from proton-like tracks. One possible variable is track length. Figure 6.24 shows the track length of proton-like tracks rejected by the requirement of at least two extended tracks. The track length of gamma rays tends to be shorter than that of protons. Although using only the track length is still hard to distinguish gamma rays

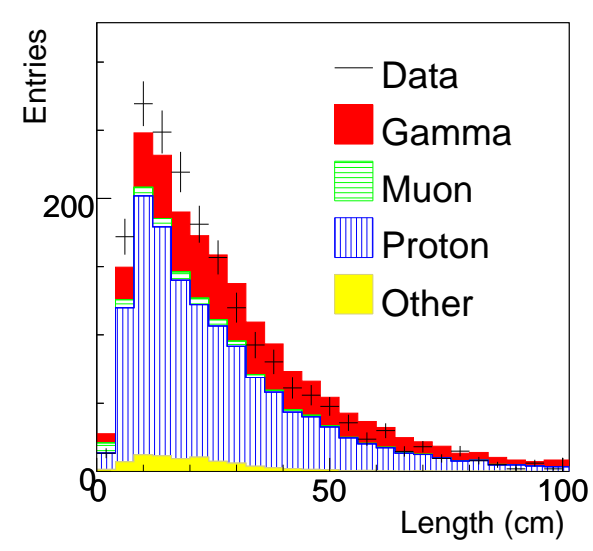

Figure 6.24: The track length of proton-like tracks rejected by requirement of at least two extended tracks.

from protons, the combination with other valuables may make it possible. For example, protons deposit larger energy near the stopping point than at the beginning. On the other hand, the maximum energy deposit in gamma rays should be located around the center of whole track since the number of $e^{-}\left(e^{+}\right)$in the electromagnetic showers (gamma rays) increases until their energies are not enough to produce another $e^{+} e^{-}$and decreases after that. Further study in this direction may enable us to distinguish gamma rays from protons.

In addition, the requirement of at least two extended tracks rejects about $90 \mathrm{NC} \pi^{0}$ signal events by identifying two back-to-back gamma rays as one gamma ray consisting of two co-linear tracks (one extended track). It may be possible to distinguish two back-toback gamma rays from one gamma ray broken into to co-linear tracks by using the track direction. This is because the two back-to-back gamma rays have opposite directions while the two co-linear tracks made by a single gamma ray have same directions. The timing 
difference between the track edges may be able to determine the track direction. Since typical gamma ray track length is roughly $30 \mathrm{~cm}$ corresponding to $1 \mathrm{~ns}$ transportation at speed of the light, the timing resolution better than 1 ns is desired. Using SciBar, we need more precise timing calibration because the SciBar timing resolution evaluated with cosmic ray data is now $1.6 \mathrm{~ns}$.

Since the current expected number of the $\mathrm{NC} \pi^{0}$ signals and the detection efficiency are 368 and $5.3 \%$ after all event selections, respectively, the detection efficiency could be increased up to $(90+140+368) / 368 \times 0.053=0.086$ if the improvement described above is possible. Considering that we can increase the gamma conversion probability up to $100 \%$ by inserting lead planes, the detection efficiency attainable in future is up to $0.086 \times(100 / 30) \sim 30 \%$. 


\section{Chapter 7}

\section{Conclusions}

The SciBooNE experiment is designed for measuring neutrino-nucleus cross sections at the neutrino energy around $1 \mathrm{GeV}$. The measurement of the $\pi^{0}$ production via $\mathrm{NC}$ interaction is the primary motivation of the SciBooNE experiment. In this thesis, we present a study of $\mathrm{NC} \pi^{0}$ production from muon neutrinos scattering on a polystyrene $\left(\mathrm{C}_{8} \mathrm{H}_{8}\right)$ target in the SciBooNE experiments using SciBooNE's full neutrino data set, corresponding to $0.99 \times 10^{20}$ protons on target.

Understanding the $\mathrm{NC} \pi^{0}$ production at the neutrino energy of $1 \mathrm{GeV}$ is essential for the neutrino oscillation experiments because the $\mathrm{NC} \pi^{0}$ production is a dominant background for $\nu_{\mu} \rightarrow \nu_{e}$ oscillation measurements. The $\nu_{\mu} \rightarrow \nu_{e}$ oscillation is not yet discovered and its corresponding mixing angle $\theta_{13}$ is constrained as $\sin ^{2} 2 \theta_{13}<0.15$ at the $90 \%$ confidence level. The new neutrino oscillation experiments such as $\mathrm{T} 2 \mathrm{~K}$ and $\mathrm{NO} \nu \mathrm{A}$ aim at the sensitivity of $\sin ^{2} 2 \theta_{13}$ below $10^{-2}$. To achieve this, the $\mathrm{NC} \pi^{0}$ production must be measured within $10 \%$ uncertainty. At the neutrino energy around $1 \mathrm{GeV}$, there are two main $\pi^{0}$ production mechanisms: resonant pion production and coherent pion production. Although several theoretical models exist to describe these $\pi^{0}$ production mechanisms including the nuclear effects, there are discrepancies among these predictions. Hence, more experimental inputs are essential. However, the cross section of the neutral current $\pi^{0}$ production was not measured within the $10 \%$ uncertainty, especially in the neutrino energy below $1 \mathrm{GeV}$. Not only the total cross section but also the kinematic distribution of the emitted $\pi^{0}$ must be measured. This is because the intra-nuclear interaction modifying the primary $\pi^{0}$ via simple neutrino-nucleon scatterings is also poorly known.

The coherent pion production have drawn much attention also in the neutrino interaction physics. This is because the Rein and Sehgal model used widely in neutrino oscillation experiments can not explain recent experimental results such as K2K, SciBooNE and MiniBooNE at the mean neutrino energy around $1 \mathrm{GeV}$. Especially, the $\mathrm{K} 2 \mathrm{~K}$ and SciBooNE experiment report null observation of the $\mathrm{CC}$ coherent pion production. Hence, it is important to measure the $\mathrm{NC}$ coherent pion production.

We have measured the cross section ratio of the $\mathrm{NC} \pi^{0}$ production to the total $\mathrm{CC}$ interaction and the $\pi^{0}$ kinematic distribution such as momentum and direction. For the cross section ratio, we obtain

$$
\frac{\sigma\left(\mathrm{NC} \pi^{0}\right)}{\sigma(\mathrm{CC})}=(7.7 \pm 0.5(\text { stat. }) \pm 0.5(\text { sys. })) \times 10^{-2} .
$$

The mean energy of neutrinos producing detected $\pi^{0} \mathrm{~s}$ is $1.1 \mathrm{GeV}$. The MC prediction 
with the Rein-Sehgal model implemented in our neutrino interaction simulation program with nuclear effects is $6.8 \times 10^{-2}$. Therefore, our result agrees with the MC prediction. We achieve less than $10 \%$ uncertainty which is required for the $\nu_{\mu} \rightarrow \nu_{e}$ oscillation measurement down to the sensitivity of $\sin ^{2} 2 \theta_{13} \sim 10^{-2}$.

The measured shapes of the $\pi^{0}$ momentum and angular distributions, as shown in Fig. 6.12 agree with the MC prediction. This means that the intra-nuclear interaction models describe our data with our uncertainty level $(\sim 10 \%)$.

We also measure the cross section ratio of the $\mathrm{NC}$ coherent pion production to the total CC interactions. We obtain

$$
\frac{\sigma\left(\mathrm{NCcoherent} \pi^{0}\right)}{\sigma(\mathrm{CC})}=(1.17 \pm 0.23) \times 10^{-2} .
$$

The mean energy of neutrinos producing detected coherent $\pi^{0} \mathrm{~s}$ is $1.0 \mathrm{GeV}$. This result is 5.8 standard deviations above the no coherent production assumption. This cross section ratio is consistent with the prediction by the Rein and Sehgal model, which is $1.21 \times 10^{-2}$. The Rein and Sehgal model also predicts the relation between the $\mathrm{CC}$ and $\mathrm{NC}$ coherent pion production: $\sigma(\mathrm{CC})=2 \times \sigma(\mathrm{NC})$. However, SciBooNE reports no observation of the $\mathrm{CC}$ coherent pion production. Hence, the Rein and Sehgal model can not explain both $\mathrm{NC}$ and $\mathrm{CC}$ coherent pion result of SciBooNE. This creates active controversies on the model of coherent pion production, and understanding of coherent pion production is in progress. 


\section{Appendix A}

\section{The Gamma and $\pi^{0}$ Reconstruction}

In this chapter, the details of $\gamma$ and $\pi^{0}$ reconstruction are described. First, we describe the method of the $\gamma$ reconstruction; extended track and SciBar-EC matching. Then, we describe the performance of the $\gamma$ and $\pi^{0}$ reconstruction including angular resolution, momentum resolution and so on.

\section{A.1 Extended Track}

In order to improve the reconstruction of $\gamma_{\mathrm{s}}$, we developed the new reconstruction algorithm called extended track. Extended tracks are reconstructed based on the tracks. Since extended tracks are used after the EC cut, the event sample used for plots in this section is after the EC cut described in Section 5.5.8.

\section{A.1.1 Track Merge}

Some of single $\gamma \mathrm{s}$ are broken into two tracks in SciBar (Figure A.1).We should reconstruct such events as a single $\gamma$ events by merging tracks on a common straight line. To do this, we fit hits associated to two tracks in each view with a straight line and we merge them if both reduced $\chi^{2} \mathrm{~s}$ are less than 50. Figure A.2 shows the distribution of the reduced $\chi^{2}$. The red color shows the case where two tracks are made by same particle (correct pairing), while the blue color shows the case where two tracks are made by different particle (wrong pairing). The 2D plots between the reduced $\chi^{2} \mathrm{~s}$ of top view and side view are shown in Figure A.3. After all event selections, about $7 \%$ of selected extended tracks are made by merged tracks.

\section{A.1.2 Energy Reconstruction}

After merging tracks, hits around the merged tracks are collected for energy reconstruction. The region where hits are collected is illustrated in Figure A.4. The energy of merged tracks are calculated as a sum of energy deposit in this region. We consider two variables to decide the size of this region ( $\mathrm{R}$ in Figure A.4). One is "energy collection efficiency" $\left(\epsilon_{\gamma}\right)$ and the other "gamma energy purity" $\left(P_{\gamma}\right)$. These two variable are defined as

$$
\epsilon_{\gamma}=\frac{\text { gamma energy in extended track }}{\text { true gamma energy }}
$$




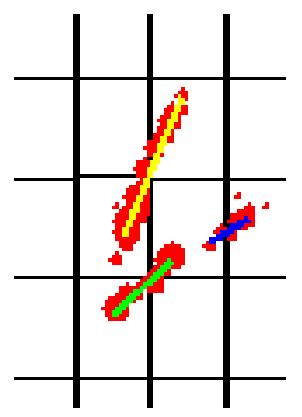

Figure A.1: An example for $2 \gamma \mathrm{s}$ from $\pi^{0}$. Red dots show hits in SciBar and colored line shows tracks. One $\gamma$ is broken into two tracks in this events
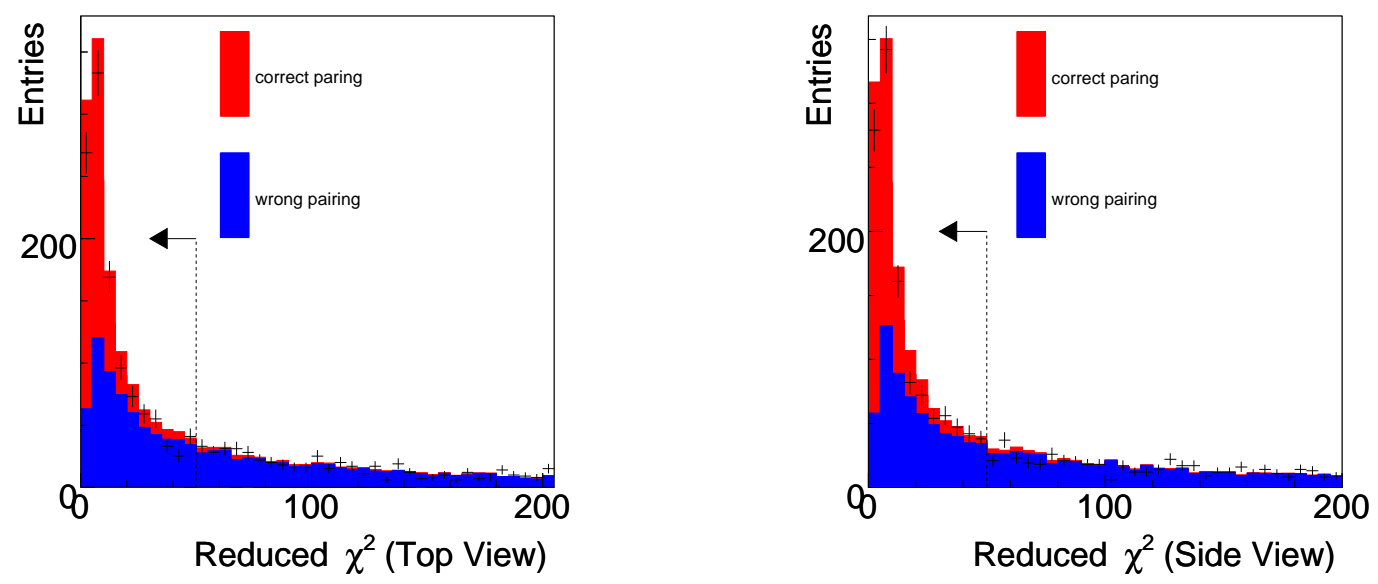

Figure A.2: Distribution of reduced $\chi^{2}$ by fitting hits in two tracks for both view. The left plot shows top view and the right shows side view. The red color shows correct pairing, while the blue color shows wrong pairing
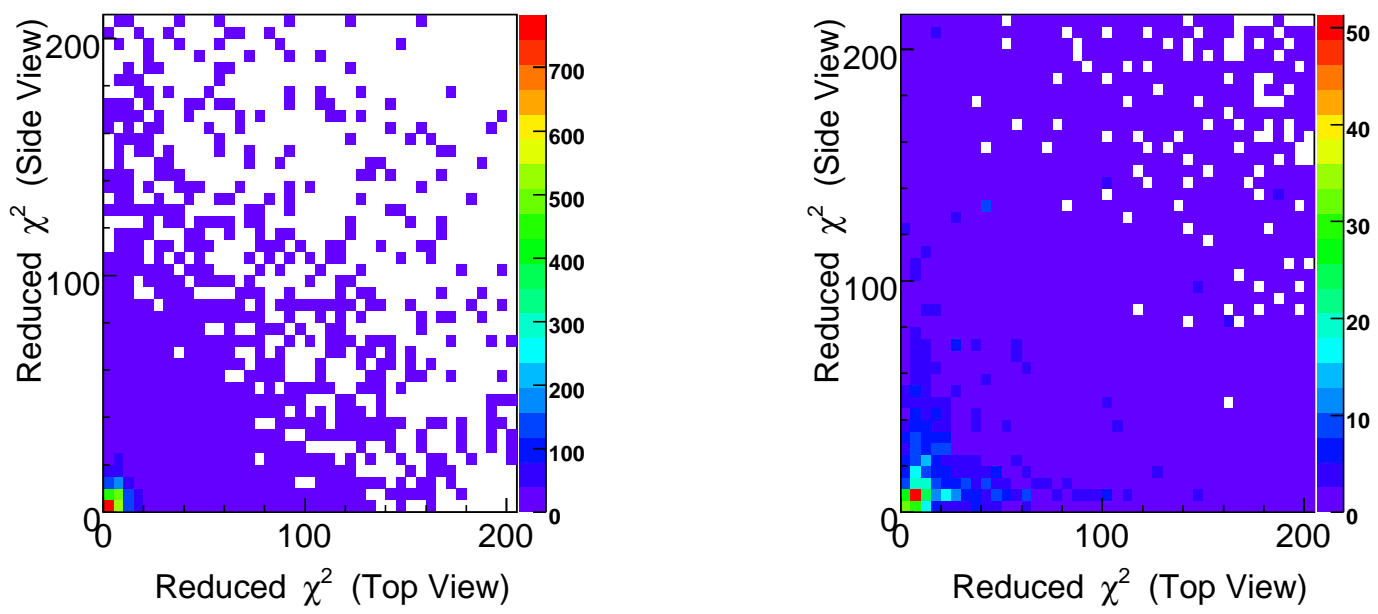

Figure A.3: 2D distribution of reduced $\chi^{2}$ by fitting hits in two tracks for both view in the MC simulation. The left plot shows correct pairing and the right shows wrong pairing. 


$$
P_{\gamma}=\frac{\text { gamma energy in extended track }}{\text { total energy in extended track }}
$$

The $\epsilon_{\gamma}$ become large with large $\mathrm{R}$ because we collect $\gamma$ hits even far from tracks. But the

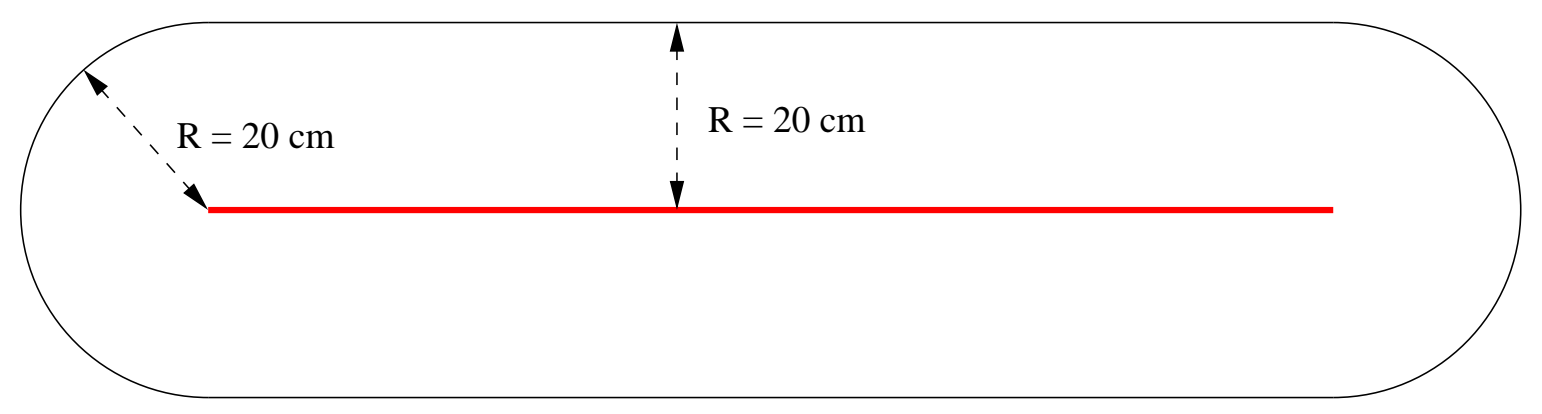

The $2 \mathrm{D}$ projection of a 3D track

Hit collection rejion

Figure A.4: Hit collection region. Hits are collected in this region for both view

$P_{\gamma}$ becomes small with large $\mathrm{R}$ because we collect hits associated with different particles near the $\gamma$ ray. Figure A.5 shows $\epsilon_{\gamma}$ and $P_{\gamma}$ dependence of "R". Since the $\epsilon_{\gamma}$ begins to saturate around $\mathrm{R}=20 \mathrm{~cm}$ but the $P_{\gamma}$ decreases linearly up to $\mathrm{R}=30 \mathrm{~cm}$, the $\mathrm{R}=20 \mathrm{~cm}$ is chosen. The performance of the energy reconstruction will be discussed in Section A.3.

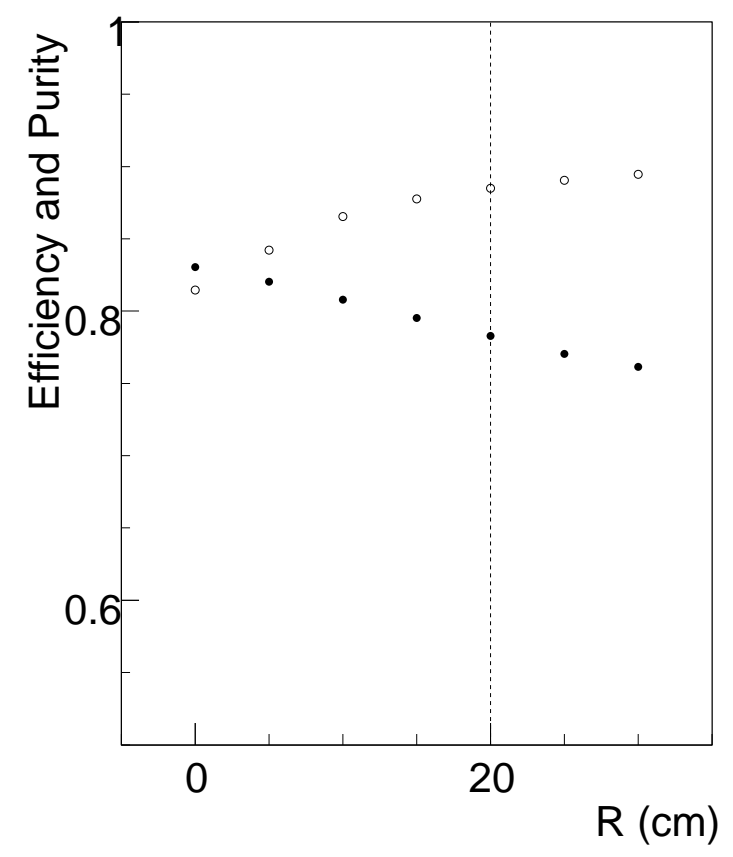

Figure A.5: $P_{\gamma}($ filled circle $)$ and $\epsilon_{\gamma}($ empty circle $)$ as a function of R shown in Figure A.4 


\section{A.1.3 Direction Reconstruction}

The reconstructed $\gamma$ direction is used for the $\pi^{0}$ vertex reconstruction, which is an important quantity in this analysis. Figure A.6 shows the number of hits around tracks for each $\mathrm{R}$ region without hits associated with tracks. (0cm $<\mathrm{R}<5 \mathrm{~cm}, 5 \mathrm{~cm}<\mathrm{R}<$ $10 \mathrm{~cm}, 10 \mathrm{~cm}<\mathrm{R}<15 \mathrm{~cm}, 15 \mathrm{~cm}<\mathrm{R}<20 \mathrm{~cm})$. The discrepancy between data and $\mathrm{MC}$ simulation become large for the large $\mathrm{R}$. Therefore, we only use information of tracks for the angle reconstruction, which means that hits newly associated in the way described in Section A.1.2 are not used for the direction reconstruction.
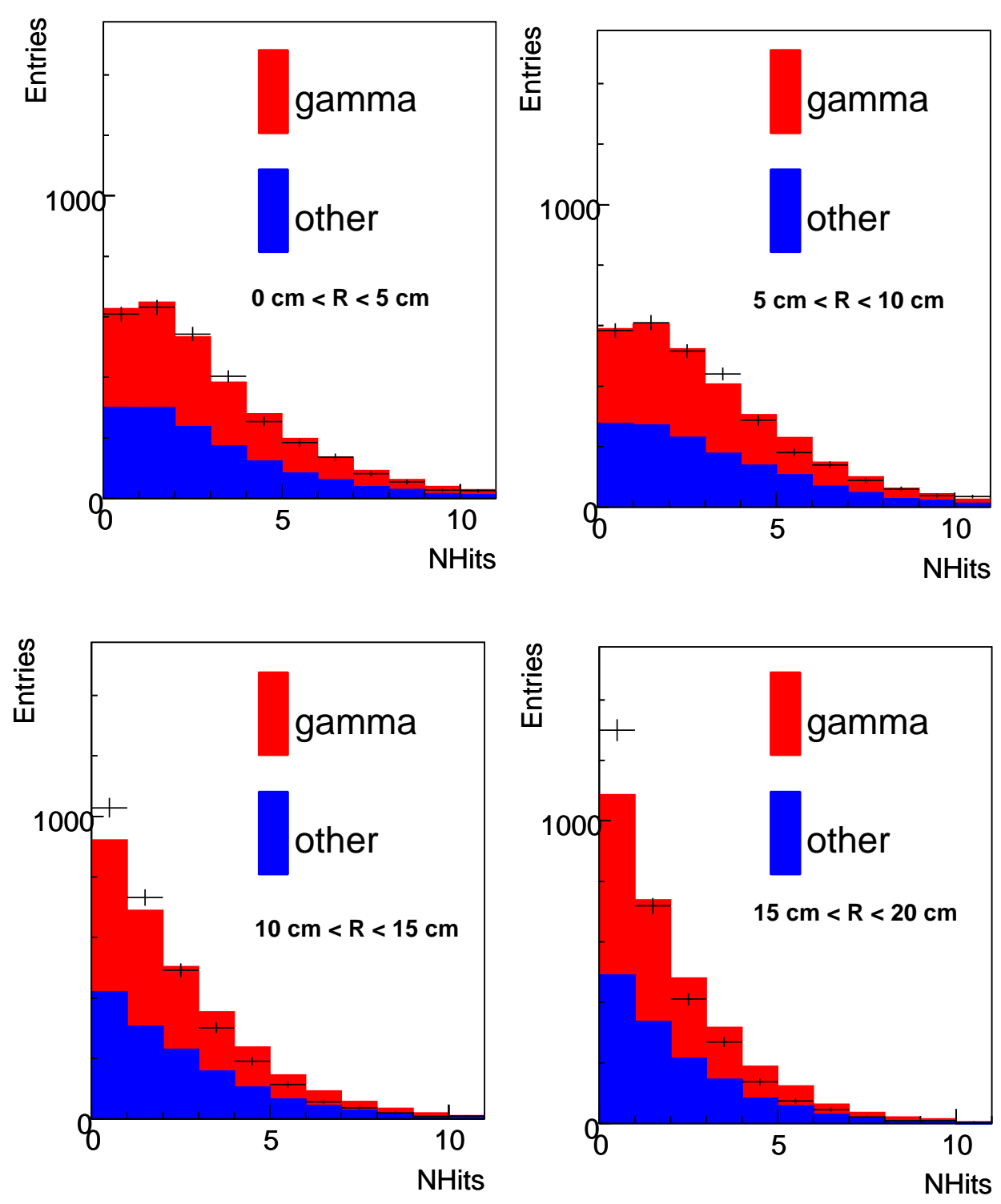

Figure A.6: The red (blue) histogram shows the distribution of the number of hits around gamma rays (other particles) for each $\mathrm{R}$ region $(0 \mathrm{~cm}<\mathrm{R}<5 \mathrm{~cm}, 5 \mathrm{~cm}<\mathrm{R}<$ $10 \mathrm{~cm}, 10 \mathrm{~cm}<\mathrm{R}<15 \mathrm{~cm}, 15 \mathrm{~cm}<\mathrm{R}<20 \mathrm{~cm})$. 


\section{A.2 SciBar-EC Matching}

Some of $\gamma$ s observed in SciBar have energy deposit in EC. After the event reconstruction in SciBar we search for EC clusters pointed by tracks from SciBar. Since we use EC information at the EC cut, the sample used for plots shown in this section is before the EC cut. The procedure of finding matched EC cluster is described below.

1. Extrapolate a track for both view and getting $\mathrm{x}$ position at $\mathrm{z}=178.4$ (EC upstream plane) ant $\mathrm{y}$ position at $\mathrm{z}=184.61 \mathrm{~cm}$ (EC downstream).

2. Take EC clusters within $10 \mathrm{~cm}$ from $\mathrm{x}, \mathrm{y}$ which are obtained above as a matched EC cluster (Figure A.7).

3. Repeat the procedure for all tracks.

Figure A.7 shows the distance between the extrapolated position and EC cluster position for gamma rays by the MC simulation and Figure A.8 are same plots for muons. The solid histogram shows the case of correct matching where EC cluster and SciBar track come from the same particle, while the dashed histogram shows the case of wrong matching where EC cluster and SciBar track made by different particle. There are peaks even for wrong matching of muons. This is the case that actual muon tracks are regarded as other particles by the algorithm matching reconstructed tracks and true particles. Matching reconstructed tracks and true particles is sometimes difficult due to high track multiplicity or overlapped tracks. Since the EC cut is for rejecting muon rather than selecting $\gamma \mathrm{s}$, we regard the EC cluster within $10 \mathrm{~cm}$ from the extrapolated position as a matched EC cluster. After all event selections, about $17 \%$ of selected extended tracks have matched EC clusters.
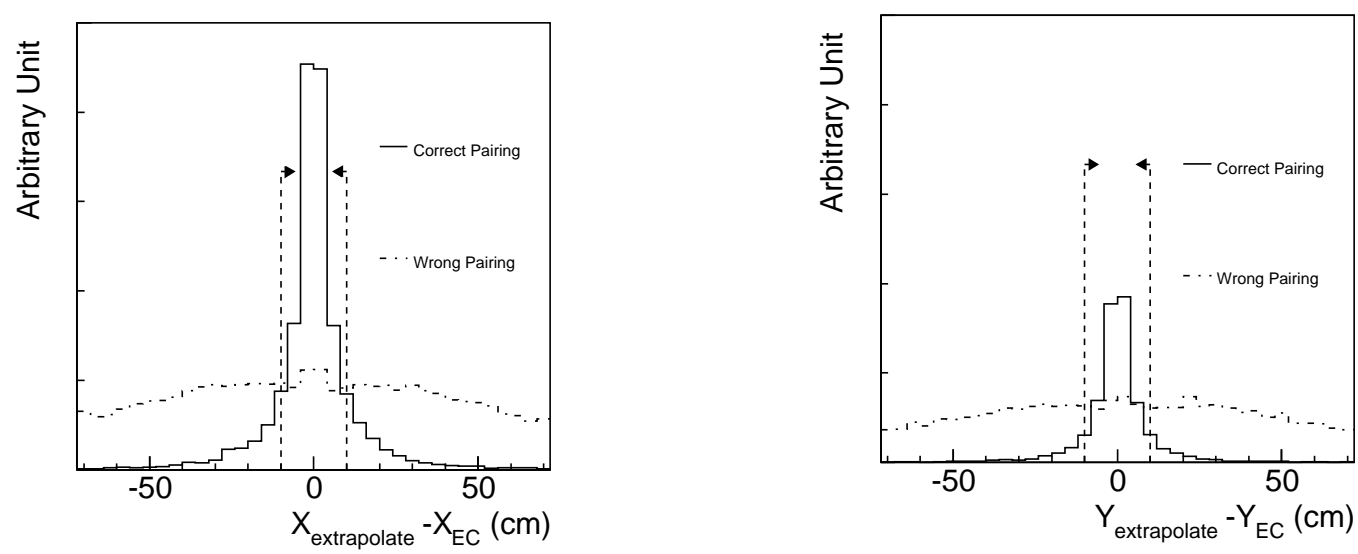

Figure A.7: The distance between the extrapolated position and EC cluster position for gamma rays in the MC simulation. The left plots shows the upstream cluster and the right shows the downstream. (Solid line:correct matching, Dashed line:wrong matching)

\section{A.3 Performance of Gamma Reconstruction}

Here we discuss the performance of $\gamma$ reconstruction. All plots in this section is after " $\pi^{0}$ mass cut" 

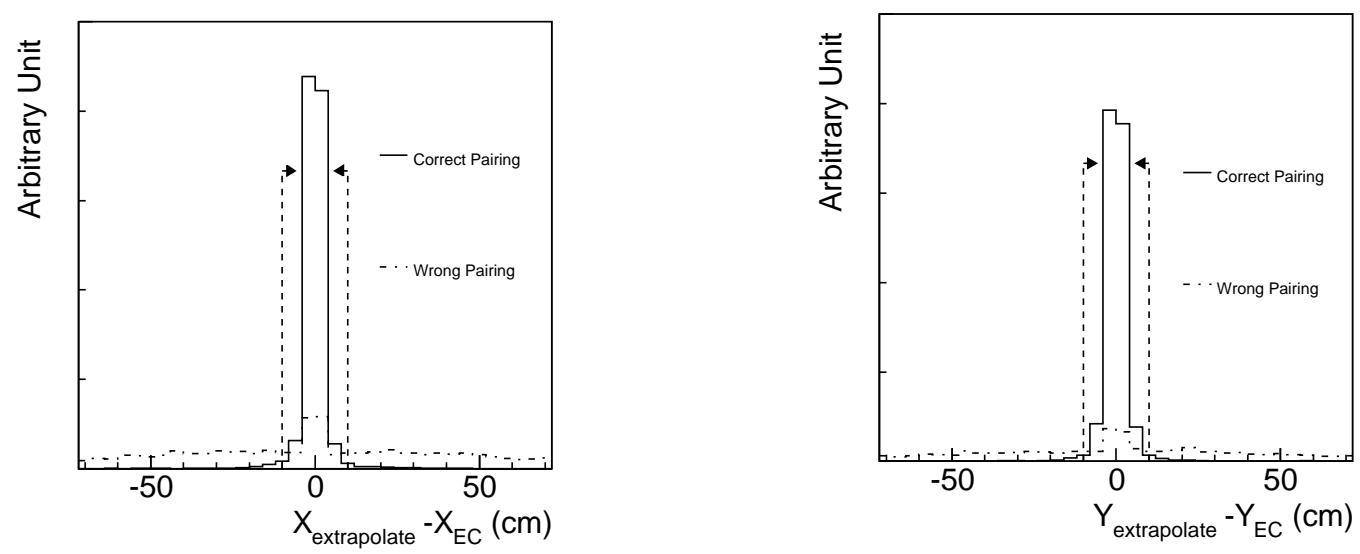

Figure A.8: The distance between the extrapolated position and EC cluster position for muons by the MC simulation. The left plots shows the upstream cluster and the right shows the downstream. (Solid line:correct matching, Dashed line:wrong matching)

\section{A.3.1 Gamma Angular Resolution}

Figure A.9 shows angles between the reconstructed $\gamma$ direction and the true $\gamma$ direction. The sigma by guassian fitting of the distribution is about 6 degree while RMS is about 14 degree. This difference comes from the long tail due to mis-reconstruction of $\gamma$ s.

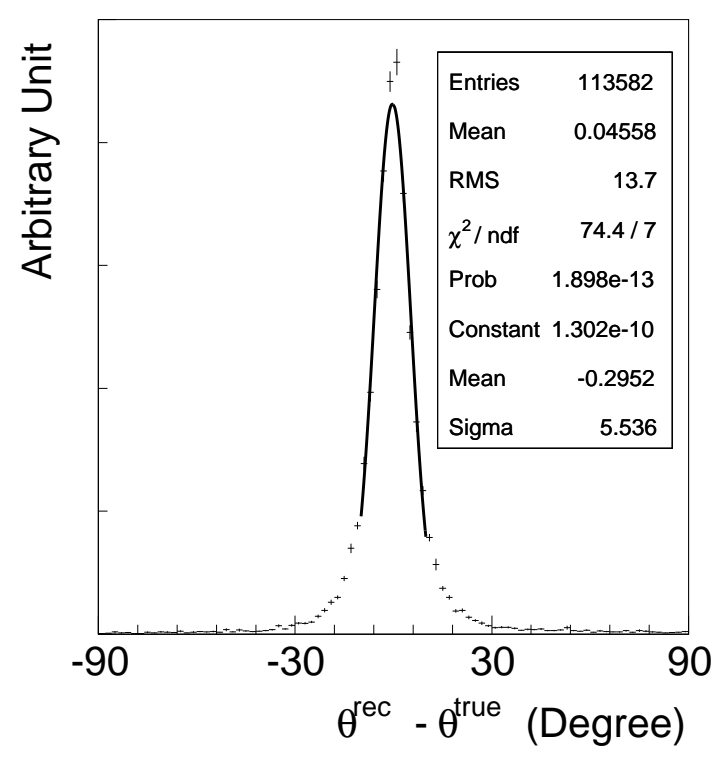

Figure A.9: The angles between reconstructed $\gamma$ direction and true $\gamma$ direction.

\section{A.3.2 Gamma Energy Resolution}

Figure A.10 shows the difference between reconstructed $\gamma$ energy in extended track and true energy deposit in SciBar. The sigma of the peak is $6.9 \mathrm{MeV}$ and the mean of $\gamma$ energy deposit in SciBar is $116 \mathrm{MeV}$ in the MC simulation. The energy resolution of $\gamma$ in SciBar is estimated to be $5.8 \%$ at $116 \mathrm{MeV}$. There is a long tail in this distribution. This 
is due to mis-reconstruction. Figure A.11 shows the difference between the reconstructed energy in the EC cluster and the true $\gamma$ energy deposit in EC. The sigma of the peak is $23 \mathrm{MeV}$ and the mean of $\gamma$ energy deposit in EC is estimated to be $72 \mathrm{MeV}$ in the MC simulation. Therefore the energy resolution of $\gamma$ in EC is estimated to be $32 \%$ at 72 $\mathrm{MeV}$.
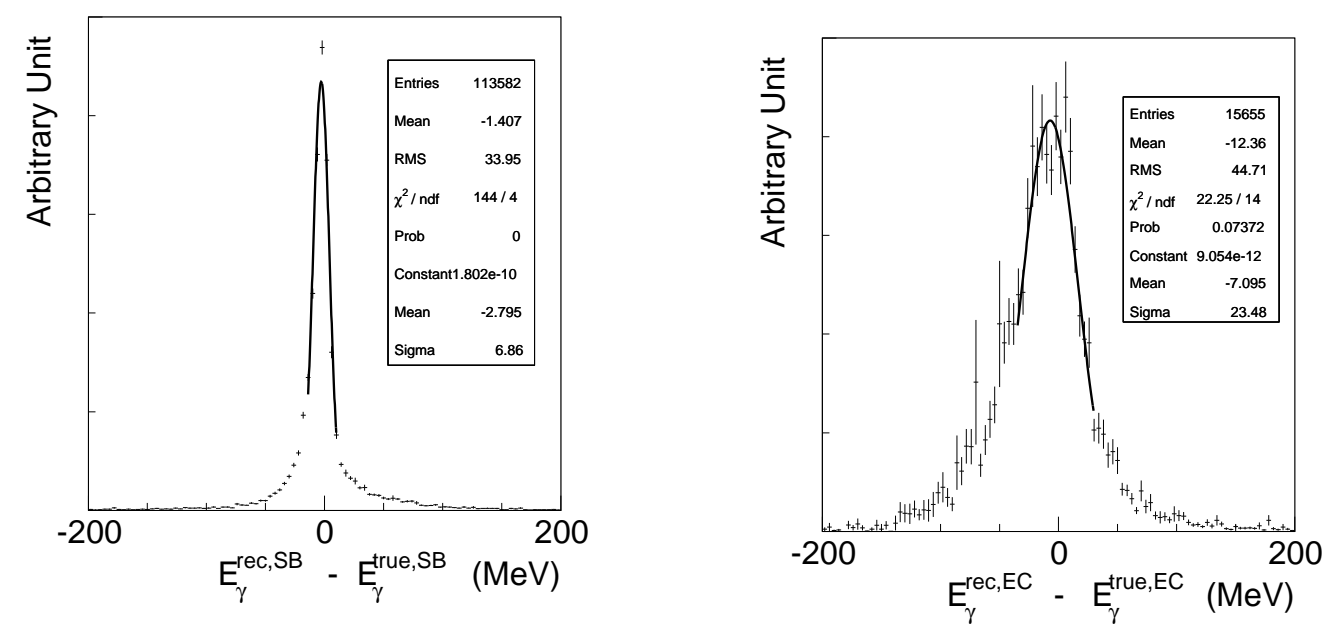

Figure A.10: The difference between the reconstructed energy in extended tracks Figure A.11: The difference between the reconstructed energy in EC cluster and and the true energy deposit by $\gamma$ in the true energy deposit by $\gamma$ in EC. SciBar.

Not all gamma ray energy is deposited in, nor recorded by, the detector. Figure A.12 shows the distribution of the actual energy collection efficiency defined as

$$
E f f_{\text {act }}=\frac{\text { gamma enengy in extended track }}{\text { true gamma energy }} \text {. }
$$

Since the average of $E f f_{a c t}$ is $76 \%$, the average lost energy is $24 \% ; 11 \%$ comes from energy loss in passive regions and gamma rays escaping from the detectors and $13 \%$ comes from energy deposit in active regions but not assigned to the extended track. The reconstructed energy could include energy deposited by other particles. Figure A.13 shows distribution of the gamma purity defined as

$$
P_{\gamma}=\frac{\text { gamma energy in the extended track }}{\text { all energy in the extended track }} .
$$

Since the average of the gamma purity is $85 \%, 15 \%$ of the total energy in an extended track comes from other particle on average. Due to this contamination, the effective energy collection efficiency defined as:

$$
E f f_{\text {eff }}=\frac{\text { reconstructed energy of extended track }}{\text { true gamma energy }} \text {. }
$$

is $85 \%$ on average, which is larger than the average of $E f f_{\text {act }}$.

Figure A.14 shows the distribution of the effective energy collection efficiency. Figure A.15 shows the 2D plot between the true gamma energy and the reconstructed energy of the extended track. The solid line on the plot shows the identity. The distribution is below the line since the effective energy collection efficiency is less than one. 


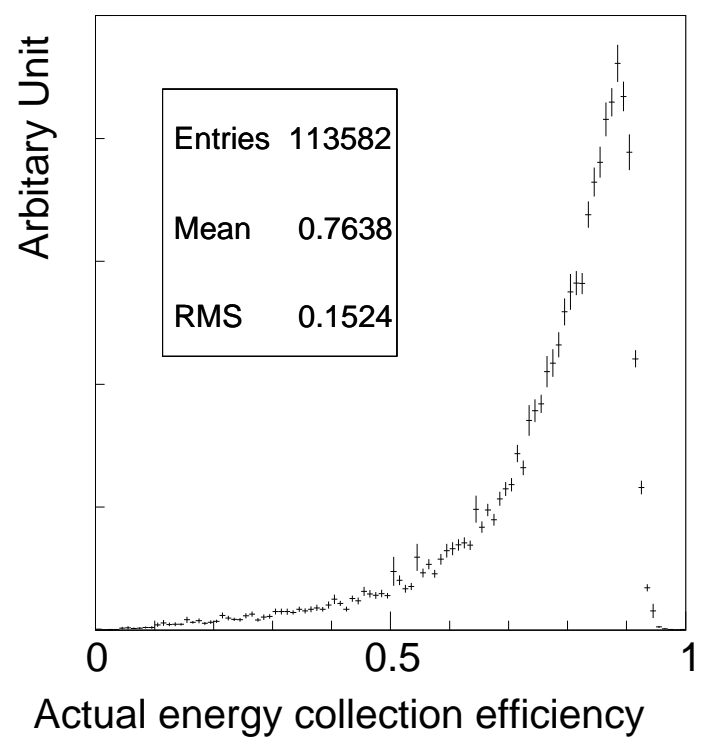

Figure A.12: Actual energy collection efficiency defined as gamma energy in extended track divided by true gamma energy.

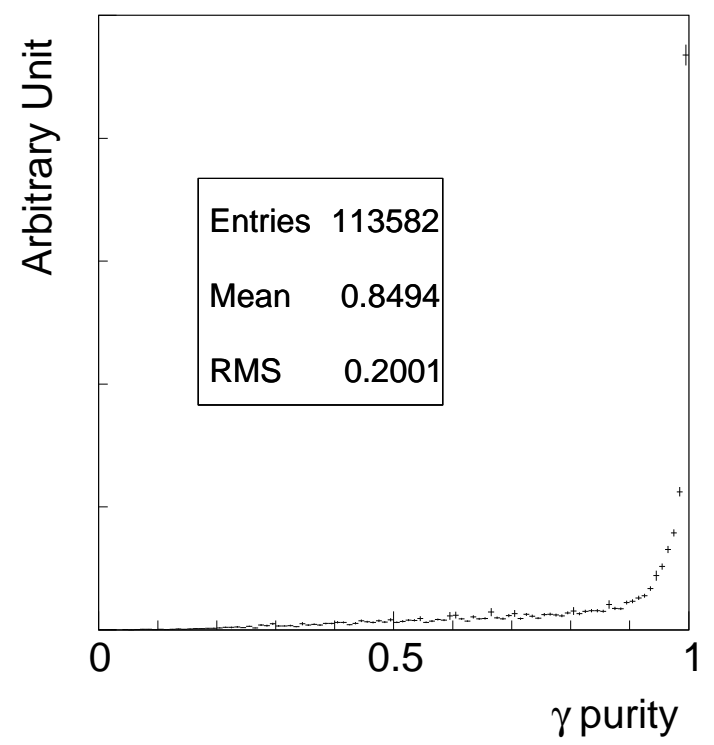

Figure A.13: Gamma purity defined as gamma energy in extended track divided by reconstructed energy of extended track. 


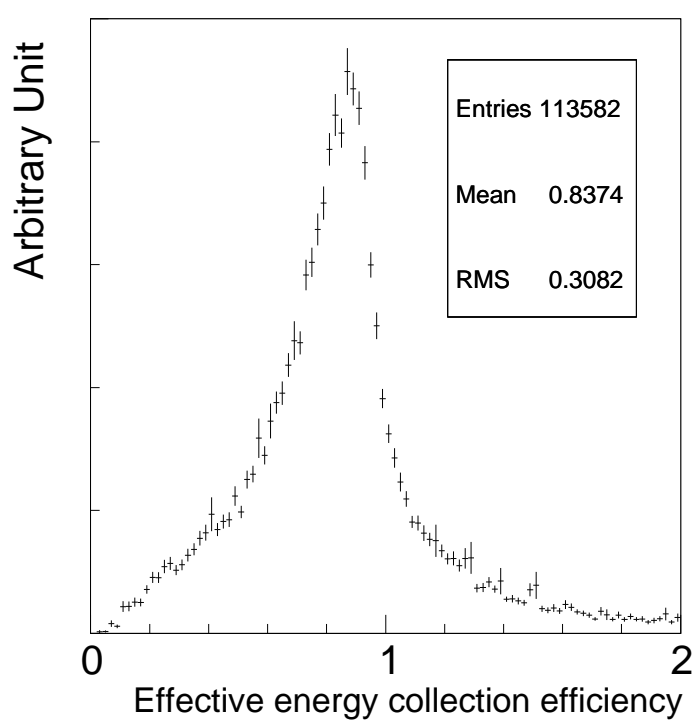

Figure A.14: Effective energy collection efficiency defined as the reconstructed energy of extended track divided by the true gamma energy.

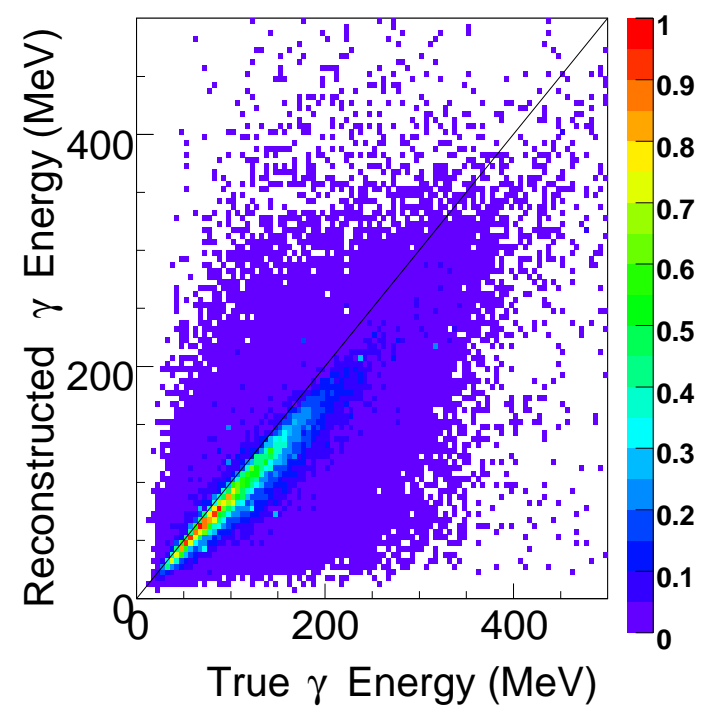

Figure A.15: 2D plot between the true $\gamma$ energy and the reconstructed energy of extended track. The line shows identity. 


\section{A.4 Performance of $\pi^{0}$ Reconstruction}

To check $\pi^{0}$ reconstruction, we look at what kind of two particles are in the selected pair of extended tracks in the $\mathrm{NC} \pi^{0}$ mode after the $\pi^{0}$ mass cut using the MC simulation. The result is shown in Table A.1. We use four types to categorize all the pairs. In the "correct $2 \gamma \mathrm{s}$ " events, both $\gamma \mathrm{s}$ from common $\pi^{0}$ are correctly associated to two extended tracks. In the "wrong $2 \gamma \mathrm{s}$ " events, both extended tracks are associated to $\gamma \mathrm{s}$ but these two are coming from different $\pi^{0} \mathrm{~s}$ or just one single $\gamma$ broken into isolated two extended tracks. In the "1 $\gamma+$ other" events, one extended track are associated to a $\gamma$ but the other extended track is associated to other charged particle. In the "two other particles" events, both extended tracks are associated to other charged particles. Possible charged particles are protons,charged pions and muons. Among these four types, Only in first one "correct $2 \gamma \mathrm{s}$ ", two extended tracks are correctly associated to $\pi^{0}$ and the contamination of this type is $87 \%$ after the $\pi^{0}$ mass cut. The plots shown in this section are using only the type "correct $2 \gamma \mathrm{s}$ " in the $\mathrm{NC} \pi^{0}$ to see the $\pi^{0}$ reconstruction performance.

\begin{tabular}{c||c}
\hline pair type & contamination $(\%)$ \\
\hline correct $2 \gamma \mathrm{s}$ & 87.3 \\
wrong $2 \gamma \mathrm{s}$ & 3.7 \\
$1 \gamma+$ other & 8.9 \\
2 others particle & 0.1 \\
\hline
\end{tabular}

Table A.1: The type of the extended track pair after the $\pi^{0}$ mass cut

\section{A.4.1 $\pi^{0}$ Vertex Resolution}

Figure A.16 shows the difference of $\pi^{0}$ vertex between the reconstructed vertex and the MC true value after the $\pi^{0}$ mass cut. The RMS is about $12 \mathrm{~cm}$ for all dimension.

\section{A.4.2 $\quad \pi^{0}$ Angle Resolution}

Figure A.17 shows the angle between the $\pi^{0}$ reconstructed direction and the MC true $\pi^{0}$ direction. RMS is about 15 degree and the sigma by gaussian fitting is about 6 degree. These values are close to the resolution of $\gamma$ angle as we expected.

\section{A.4.3 $\pi^{0}$ Mass Resolution and Momentum Resolution}

Figure A.18 shows the difference between the reconstructed $\pi^{0}$ mass and MC true $\pi^{0}$ mass $\left(135 \mathrm{MeV} / c^{2}\right)$ before the $\pi^{0}$ mass cut. The peak are negative because we do not correct the energy leakage of $\gamma \mathrm{s}$. The sigma by gaussian fitting is $28 \mathrm{MeV} / c^{2}$ and the mean reconstructed mass is $119 \mathrm{MeV} / c^{2}$. Therefore, the average reconstructed mass resolution is about $23 \%$.

Figure A.19 shows the difference between the reconstructed $\pi^{0}$ momentum and the MC true $\pi^{0}$ momentum after the $\pi^{0}$ mass cut. The peak are negative because of energy leakage of $\gamma \mathrm{s}$. The sigma by gaussian fitting is $37 \mathrm{MeV} / c^{2}$ and the mean reconstructed momentum is $223 \mathrm{MeV} / c$. Therefore, The average momentum resolution is about 17 


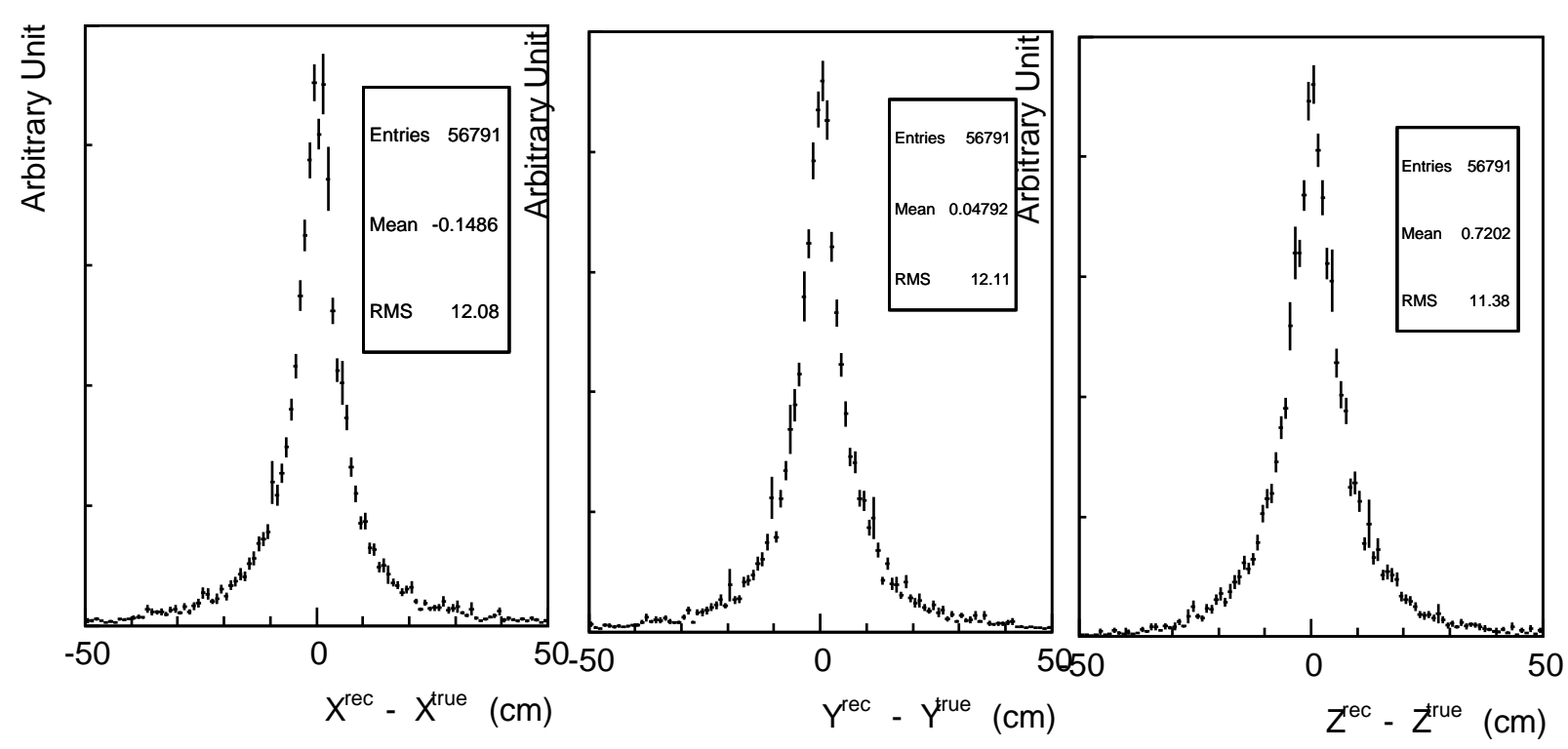

Figure A.16: The difference of $\pi^{0}$ vertex between the reconstructed $\pi^{0}$ vertex and the true value for each coordinates after the $\pi^{0}$ mass cut. From the left, $\mathrm{x}, \mathrm{y}$ and $\mathrm{z}$ vertices are shows, respectively.

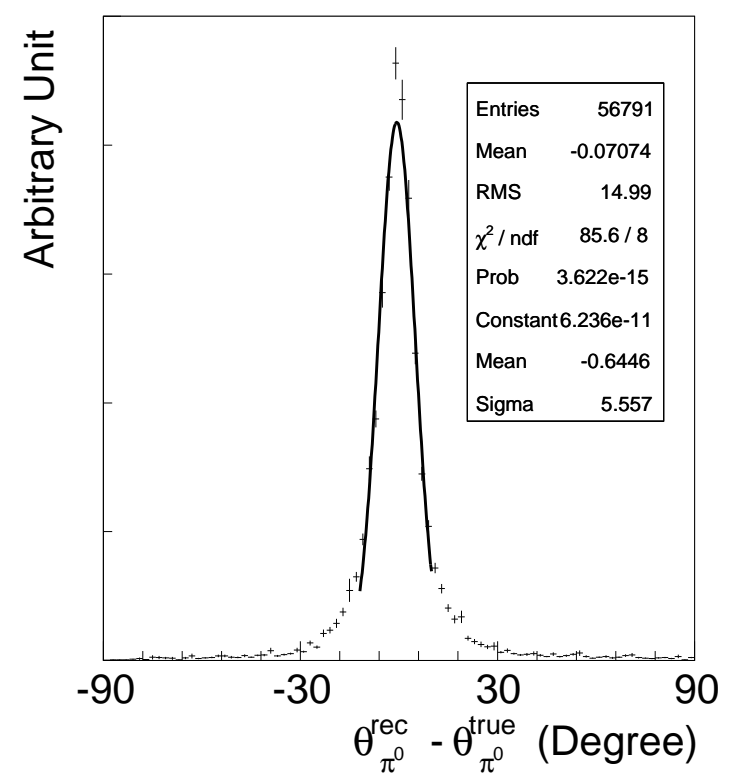

Figure A.17: The angle between the reconstructed $\pi^{0}$ direction and the MC true $\pi^{0}$ direction after the $\pi^{0}$ mass cut 


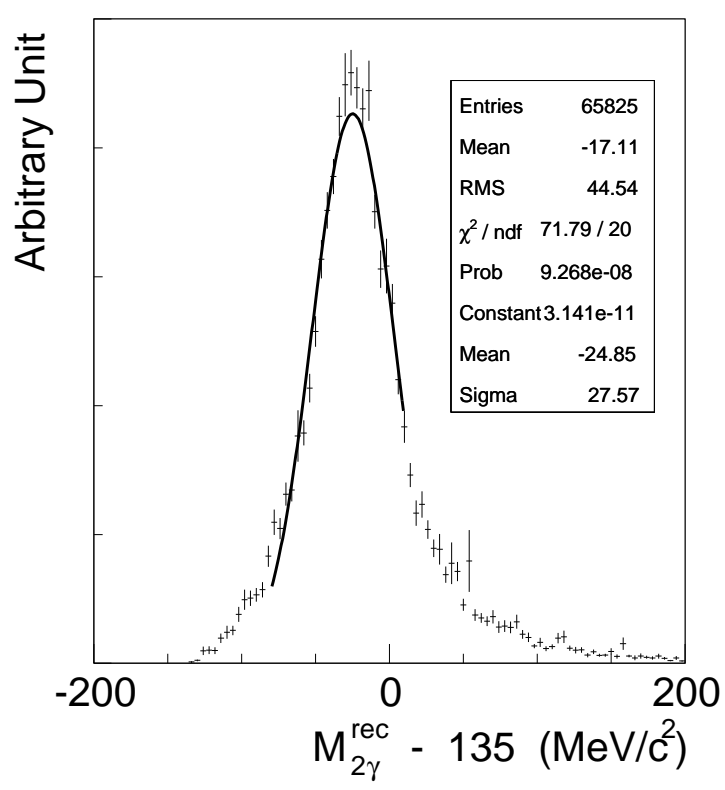

Figure A.18: The difference between the reconstructed $\pi^{0}$ mass and MC true $\pi^{0}$ mass $\left(135 \mathrm{MeV} / c^{2}\right)$ before the $\pi^{0}$ mass cut

\%. We already show the relation between the $\pi^{0}$ true momentum and the reconstructed momentum after the $\pi^{0}$ mass cut in Figure 6.10. In the end, we put the summary table of the $\pi^{0}$ reconstruction performance in Table A.2.

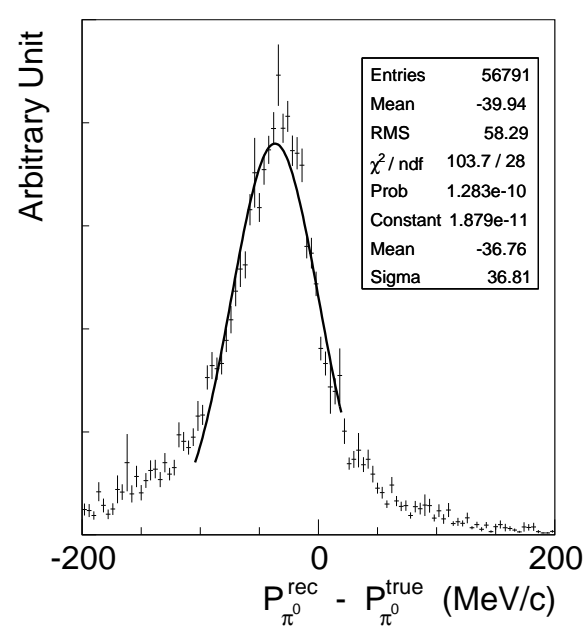

Figure A.19: The difference between the reconstructed $\pi^{0}$ momentum and the MC true $\pi^{0}$ momentum after the $\pi^{0}$ mass cut 


\begin{tabular}{c||c}
\hline$\pi^{0}$ angle resolution & 6 degree $($ sigma $)$ \\
mean $\pi^{0}$ reconstructed mass & $119 \mathrm{MeV} / c^{2}$ \\
$\pi^{0}$ mass resolution & $23 \%(\mathrm{sigma})$ \\
mean $\pi^{0}$ reconstructed momentum & $223 \mathrm{MeV} / \mathrm{c}$ \\
$\pi^{0}$ momentum resolution & $17 \%(\mathrm{sigma})$ \\
\hline
\end{tabular}

Table A.2: The performance the of $\pi^{0}$ reconstruction 


\section{Appendix B}

\section{Neutrino Interaction In The Surrounding Material}

Neutrino interactions occur outside of the detector such as the wall of the detector hall or dirt and produce secondary particles. The secondary particles by such interactions sometimes make hits in the SciBooNE detectors. We call these events "dirt background events". For NC $\pi^{0}$ measurement, neutrino induced $\pi^{0}$ production outside of the detector such as wall of the detector hall or dirt is a background. The estimate of the number of dirt background events in the $\mathrm{NC} \pi^{0}$ sample by the MC simulation is important in this analysis.

\section{B.1 Dirt material}

We use concrete as material for both the wall and dirt in the MC simulation. The contamination of each element is shown in Tab. B.1 The density is $2.15 \mathrm{~g} / \mathrm{cm}^{3}$. This estimation is very rough. Therefore we vary by $\pm 20 \%$ this density when we estimate systematic uncertainty.

\begin{tabular}{cc}
\hline \hline element & contamination (\%) \\
\hline $\mathrm{H}$ & 1 \\
$\mathrm{O}$ & 53 \\
$\mathrm{Na}$ & 2 \\
$\mathrm{Al}$ & 3 \\
$\mathrm{Si}$ & 34 \\
$\mathrm{~K}$ & 1 \\
$\mathrm{Ca}$ & 4 \\
$\mathrm{Fe}$ & 1 \\
\hline \hline
\end{tabular}

Table B.1: The contamination of each element of our concrete 


\section{B.2 The area of neutrino events}

Figure B.1 shows the area where neutrino events are generated. For the neutrino cross section, we use NEUT for $\mathrm{C}_{8} \mathrm{H}_{8}$, which is same for SciBar. About the coordination, $\mathrm{X}, \mathrm{y}$ and $\mathrm{z}$ correspond to the horizontal, vertical and beam direction, respectively. The origin of $\mathrm{x}$ and $\mathrm{y}$ is the center of SciBar and the origin of $\mathrm{z}$ is the upstream surface of SciBar. We generate neutrino events in the region, which is $-5 \mathrm{~m}<x<5 \mathrm{~m},-5 \mathrm{~m}<y<5 \mathrm{~m}$ and $-5 \mathrm{~m}<z<5 \mathrm{~m}$ for dirt MC simulation. Figure B.2 shows the vertex of neutrino
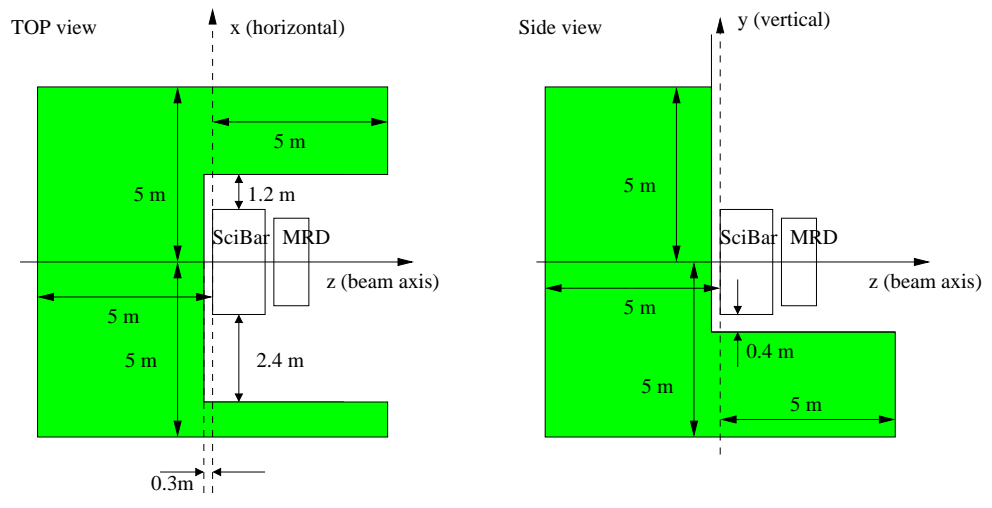

The region where dirt events are generated

Figure B.1: The area where neutrino events are generated (green region)

interactions by the dirt MC simulation after all event selections. By Fig. B.2, the most of neutrino interactions occur between $\pm 2 \mathrm{~m}$ for all directions. Therefore, The region $( \pm 5 \mathrm{~m}$ for $\mathrm{x}, \mathrm{y}$ and $\mathrm{z}$ ) is large enough.
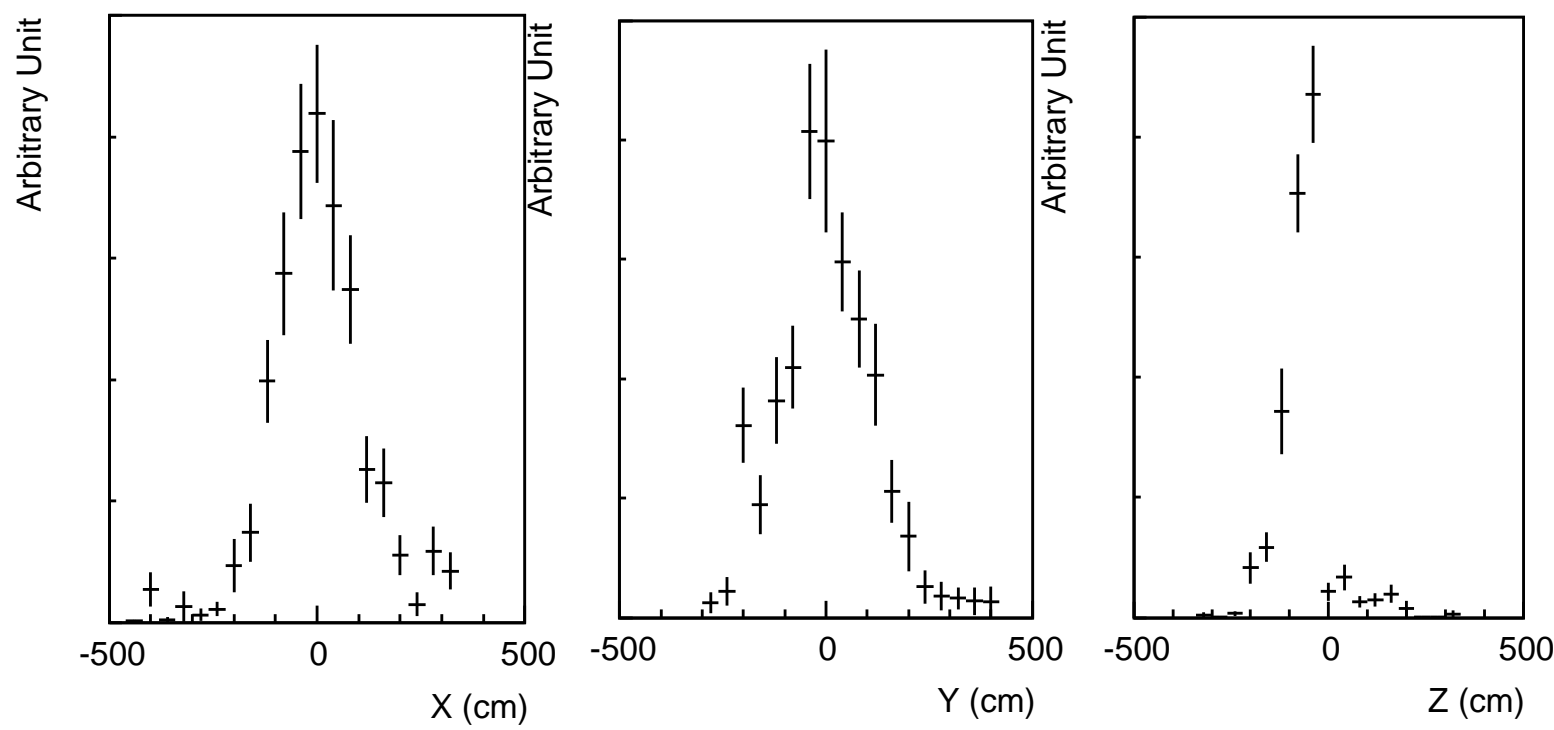

Figure B.2: The neutrino vertex of dirt MC after all event selections for $\mathrm{NC} \pi^{0}$ 


\section{Appendix C}

\section{Expectation with Systematic Uncertainty}

Some of histograms are shown with systematic errors for each bin in this thesis. In this chapter, we describe how we calculate the size of systematic errors in such histograms.

To include the systematic uncertainty, we generate 1000 expectations within systematic uncertainty and look at the variation of the value which we want to measure. For simplicity, we consider the case that we measure two value $\left(N_{1}, N_{2}\right)$ with two independent systematic source tagged as $A, B$. Since we have two sets of the systematic variations tagged as,+- (for example, MC sets with larger and smaller cross talk). we can get

$$
N_{1}^{A+}, N_{1}^{A-}, N_{1}^{B+}, N_{1}^{B-}
$$

and

$$
N_{2}^{A+}, N_{2}^{A-}, N_{2}^{B+}, N_{2}^{B-}
$$

for $N_{1}$ and $N_{2}$ (nominal MC expectations), respectively. Using them we define

$$
\begin{aligned}
& \sigma_{1}^{A+}=N_{1}^{A+}-N_{1} \\
& \sigma_{1}^{A-}=N_{1}^{A-}-N_{1} \\
& \sigma_{1}^{B+}=N_{1}^{B+}-N_{1} \\
& \sigma_{1}^{B-}=N_{1}^{B-}-N_{1} \\
& \sigma_{2}^{A+}=N_{2}^{A+}-N_{2} \\
& \sigma_{2}^{A-}=N_{2}^{A-}-N_{2} \\
& \sigma_{2}^{B+}=N_{2}^{B+}-N_{2} \\
& \sigma_{2}^{B-}=N_{2}^{B-}-N_{2} .
\end{aligned}
$$

Then, we generate random numbers of $\Delta_{1, i}^{A}, \Delta_{1, i}^{B}, \Delta_{2, i}^{A}$ and $\Delta_{2, i}^{B}$ for $i$-th generation $(0<$ $i<1000)$. To obtain $\Delta_{1, i}^{A}$ and $\Delta_{2, i}^{A}$, we get $x_{i}$ using a gaussian random number generator ( Mean $=0, \operatorname{RMS}=1$ ).

If we get $x_{i}>0$

$$
\begin{aligned}
& \Delta_{1, i}^{A}=\sigma_{1}^{A+} \times x_{i} \\
& \Delta_{2, i}^{A}=\sigma_{2}^{A+} \times x_{i}
\end{aligned}
$$


otherwise,

$$
\begin{aligned}
& \Delta_{1, i}^{A}=\sigma_{1}^{A-} \times x_{i} \\
& \Delta_{2, i}^{A}=\sigma_{2}^{A-} \times x_{i} .
\end{aligned}
$$

To obtain $R_{1, i}^{B}$ and $R_{2, i}^{B}$, we get $y_{i}$ using a gaussian random number generator ( Mean = 0 , RMS $=1)$. The $y_{i}$ must be obtained by the different random number generation for $x_{i}$ since the systematic sources of $A$ and $B$ are independent from each other. If we get $y_{i}>0$

$$
\begin{aligned}
& \Delta_{1, i}^{B}=\sigma_{1}^{B+} \times y_{i} \\
& \Delta_{2, i}^{B}=\sigma_{2}^{B+} \times y_{i}
\end{aligned}
$$

otherwise,

$$
\begin{aligned}
& \Delta_{1, i}^{B}=\sigma_{1}^{B-} \times y_{i} \\
& \Delta_{2, i}^{B}=\sigma_{2}^{B-} \times y_{i} .
\end{aligned}
$$

Finally we obtain $i$-the expectation for $N_{1}$ and $N_{2}$ expressed as

$$
\begin{aligned}
& N_{1, i}=N_{1}+\Delta_{1, i}^{A}+\Delta_{1, i}^{B} \\
& N_{2, i}=N_{2}+\Delta_{2, i}^{A}+\Delta_{2, i}^{B}
\end{aligned}
$$

respectively. When we obtain the RMS of $F\left(N_{1}, N_{2}\right)$ (some function of $N_{1}$ and $N_{2}$ ), we calculate

$$
\Delta F\left(N_{1}, N_{2}\right)=\sqrt{\frac{1}{1000} \sum_{i=1}^{1000}\left(F\left(N_{1, i}, N_{2, i}\right)-\hat{F}\left(N_{1}, N_{2}\right)\right)^{2}}
$$

respectively. We use $\Delta F\left(N_{1}, N_{2}\right)$ as the size of systematic error box. 


\section{Appendix D}

\section{Charged Current Coherent Pion Production}

The SciBooNE collaboration reports no observation of CC coherent pion production [49]. However, as described in Section 6.6.1, further study reveals the data excess comparing to the zero coherent pion prediction in the forward pion sample $\left(\theta_{\pi}<35^{\circ}\right)$ both for neutrino and antineutrino data. In this chapter, we present the summary of recent study of CC coherent pion production [101,102]. For analyses presented in this chapter, NEUT is used for the neutrino interaction simulation. Hence, the Rein and Sehgal model is employed for the coherent pion production.

\section{D.1 Event Selection of CC Coherent Pion Produc- tion}

The same event selection cuts are applied for neutrino and antineutrino CC coherent pion search. Although the MC tuning is performed in the neutrino mode, it is not yet done in the antineutrino mode.

We use the MRD-stopped event sample described in Section 5.4 as the CC event sample. After the CC selection, we select events with one additional track originating from the common vertex of the SciBar-MRD matched track. This additional track is required to be a pion-like track which means MuCL > 0.05 (Muon Confidence Level, Section 5.3.3). The recoil proton in the incoherent pion production can be identified by using the energy deposit around the vertex (vertex activity). The CC coherent pion events do not have the vertex activity. We also reduce the $\mathrm{CC}$ quasi-elastic events by using the angle $\left(\Delta \theta_{p}\right)$ between the expected proton track and the observed second track directions. In case of CC quasi-elastic events, the proton angle can be predicted by the muon angle and momentum, so that we expect $\Delta \theta_{p} \sim 0$. In case of non-elastic events such as the pion production, we expect $\Delta \theta_{p} \neq 0$. Finally, we select events with the pion track candidate directed forward, which is also the characteristic of coherent pion production. The details of the event selection cuts are described in $[49,56]$. 


\section{D.2 Study of CC Coherent Pion Production for Neu- trino and Antineutrino}

\section{D.2.1 $\nu_{\mu}$ CC Coherent Pion Production}
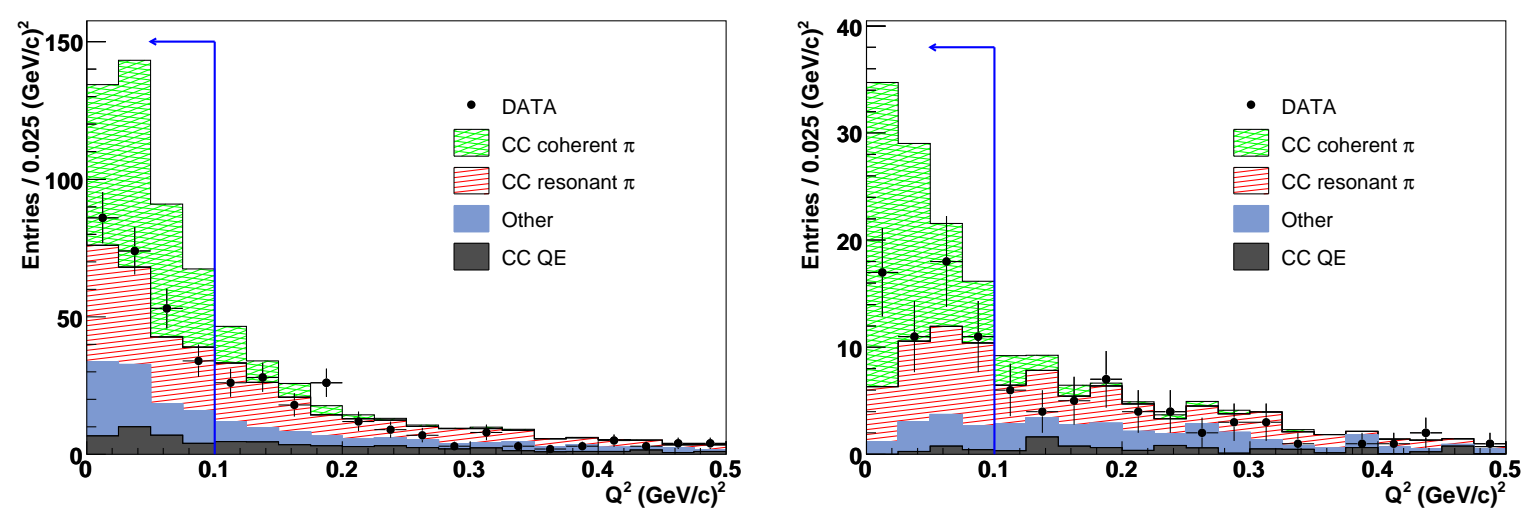

Figure D.1: The $Q_{\text {rec }}^{2}$ distribution for the MRD stopped CC coherent pion sample (left), and the MRD penetrated CC coherent pion sample (right).

Figure D.1 (left) shows the $Q_{\text {rec }}^{2}$ distribution for the selected events. The $Q_{\text {rec }}^{2}$ is called a reconstructed $Q^{2}$ calculated as

$$
Q_{\mathrm{rec}}^{2}=2 E_{\nu}^{\mathrm{rec}}\left(E_{\mu}-p_{\mu} \cos \theta_{\mu}\right)-m_{\mu}^{2}
$$

where $E_{\nu}^{\mathrm{rec}}$ is the reconstructed neutrino energy calculated by assuming CC quasi-elastic kinematics,

$$
E_{\nu}^{\mathrm{rec}}=\frac{1}{2} \frac{\left(M_{p}^{2}-m_{\mu}^{2}\right)-\left(M_{n}-V\right)^{2}+2 E_{\mu}\left(M_{n}-V\right)}{\left(M_{n}-V\right)-E_{\mu}+p_{\mu} \cos \theta_{\mu}}
$$

where $M_{p}$ and $M_{n}$ are the mass of proton and neutron, respectively, and $V$ is the nuclear potential, which is set to $27 \mathrm{MeV}$. In the signal region with $Q_{\text {rec }}^{2}$ less than $0.1 \mathrm{GeV} / c^{2}, 247$ $\mathrm{CC}$ coherent pion candidate events are observed, while the expected number of background events is $228 \pm 12$. The selection efficiency for the signal is estimated to be $10.4 \%$. The mean neutrino beam energy for true CC coherent pion events in the sample is estimated to be $1.1 \mathrm{GeV}$ after accounting for the effects of the selection efficiency.

We also use the MRD penetrated sample to search for CC coherent pion production at higher energy. Events with the SciBar-MRD matched track not stopping in MRD but exiting from the downstream end of MRD are defined as the MRD penetrated sample. The same selection cuts are applied to the MRD penetrated sample to search for CC coherent pion candidate events. Figure D.1 (right) shows the $Q_{\text {rec }}^{2}$ distribution for the $\mathrm{MRD}$ penetrated $\mathrm{CC}$ coherent pion sample. In the signal region, $57 \mathrm{CC}$ coherent pion candidates are observed, while the expected number of background events is $40 \pm 2.2$. The selection efficiency for the signal is estimated to be $3.1 \%$. The mean neutrino beam energy for true CC coherent pion events in the sample is estimated to be $2.2 \mathrm{GeV}$.

Since the data excess to the background level is small for both samples, we set $90 \%$ confidence level upper limit on the cross section ratio of the $\mathrm{CC}$ coherent pion production 
to the total $\mathrm{CC}$ interaction at $0.67 \times 10^{-2}$ for a mean neutrino energy of $1.1 \mathrm{GeV}$ and $1.36 \times 10^{-2}$ for that of $2.2 \mathrm{GeV}$. These limits correspond to $33 \%$ and $67 \%$ of the prediction by the Rein and Sehgal model at $1.1 \mathrm{GeV}$ and $2.2 \mathrm{GeV}$, respectively. These limits are published in [49].

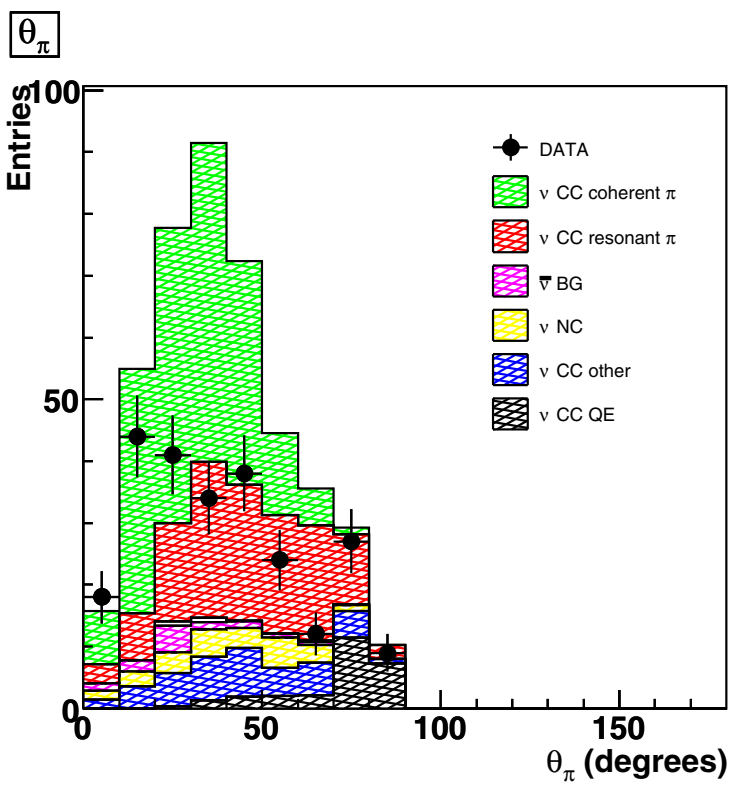

Figure D.2: The pion angular distribution in CC coherent pion sample.

After we published the upper limits, further study is performed. Figure D.2 shows the distribution of the pion angle with respect to the beam axis $\left(\theta_{\pi}\right)$ for the MRD stopped coherent sample. There are data excess with respect to the background prediction at small pion scattered angle. Motivated by this fact, both samples for neutrino and antineutrino is further divided into two sub-samples based on $\theta_{\pi}: \theta_{\pi}<30^{\circ}$ and $\theta_{\pi}>30^{\circ}$. In the case of $\mathrm{CC}$ coherent pion production, muon and pion are expected to be emitted back-to-back in the $x-y$ plane because of the conservation of momentum. Therefore, the kinematic variable called $\Delta \phi$ is defined as shown in Figure D.3. The coherent pion events are expected to distribute around $\Delta \phi=0$. Figure D.4 shows $\Delta \phi$ distributions in each sub-sample for

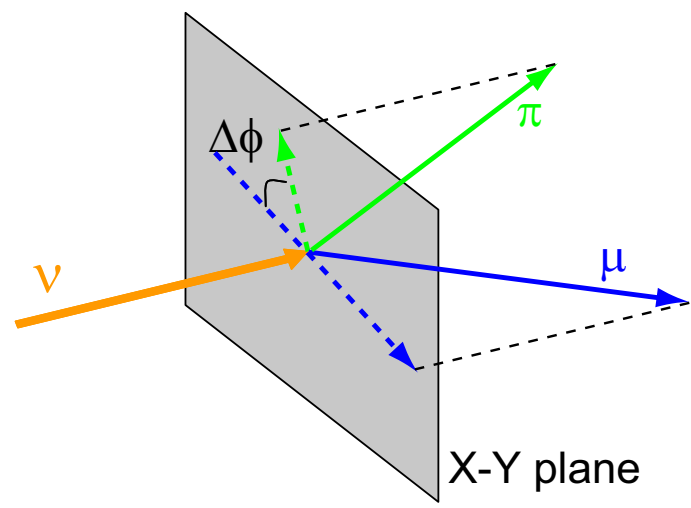

Figure D.3: Definition of the kinematic variable $\Delta \phi$. 

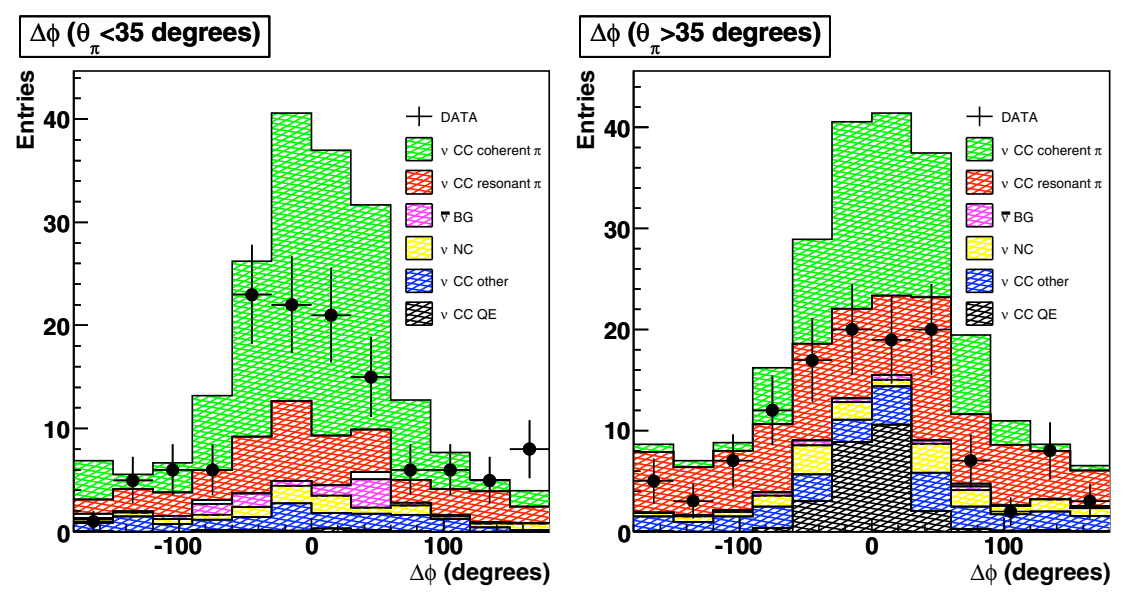

Figure D.4: $\Delta \phi$ distributions for two different regions of the pion scattering angle in CC coherent pion sample. The left plot is for $\theta_{\pi}<35^{\circ}$ and the right plot is for $\theta_{\pi} \geq 35^{\circ}$.

neutrino. In the large pion angle sample, the data and $\mathrm{MC}$ background prediction agree well. However, a data excess is found around $\Delta \phi=0$ in the small pion angle sample. This fact indicates the coherent pion signal in the region of the small pion scattering angle.

\section{D.2.2 $\bar{\nu}_{\mu}$ CC Coherent Pion Production}

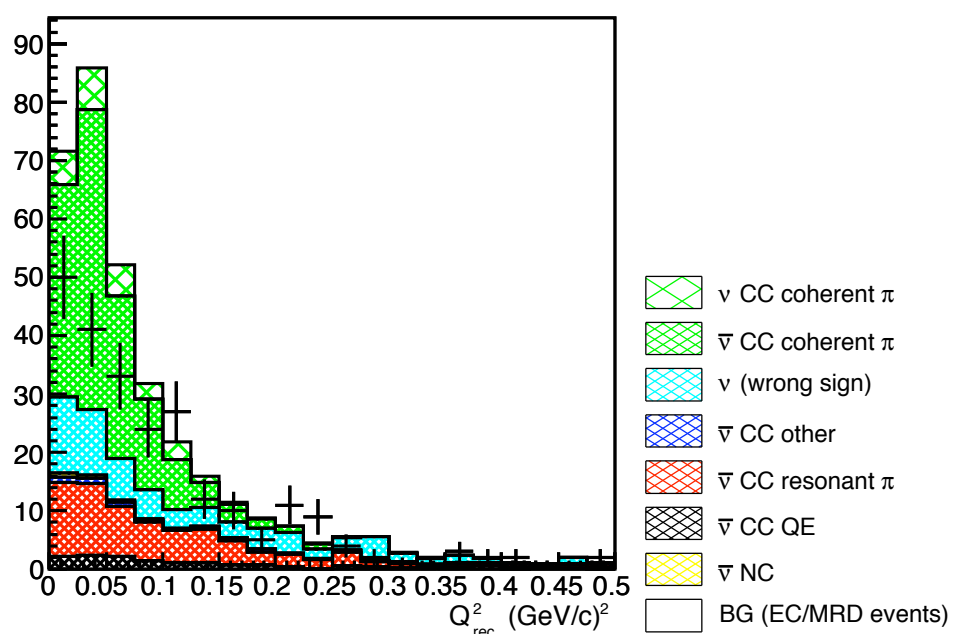

Figure D.5: The $Q_{\mathrm{rec}}^{2}$ distribution in antineutrino CC coherent pion sample.

Figure D.5 shows the $Q_{\text {rec }}^{2}$ distribution in the antineutrino CC coherent pion sample. As shown in Figure D.5, there is a data deficit in the low $Q_{\text {rec }}^{2}$ region similar to Figure D.1. However, the data clearly lie above the MC predicted background level in the coherent pion region below $0.1(\mathrm{GeV} / \mathrm{c})^{2}$, although the observed data excess is smaller than the predicted coherent pion signal. Although the systematic uncertainties are not yet included, the statistical significance of the data excess is $4 \sigma$ above the background prediction. The data excess above the predicted backgrounds are consistent with the K2K 
and SciBooNE upper limits observed in the neutrino mode within the statistical uncertainties considering the fact that the cross sections of coherent pion production are same for neutrinos and antineutrinos while the cross section of the background processes such as CC quasi-elastic interaction and resonant pion production for antineutrinos is smaller than that for neutrinos.

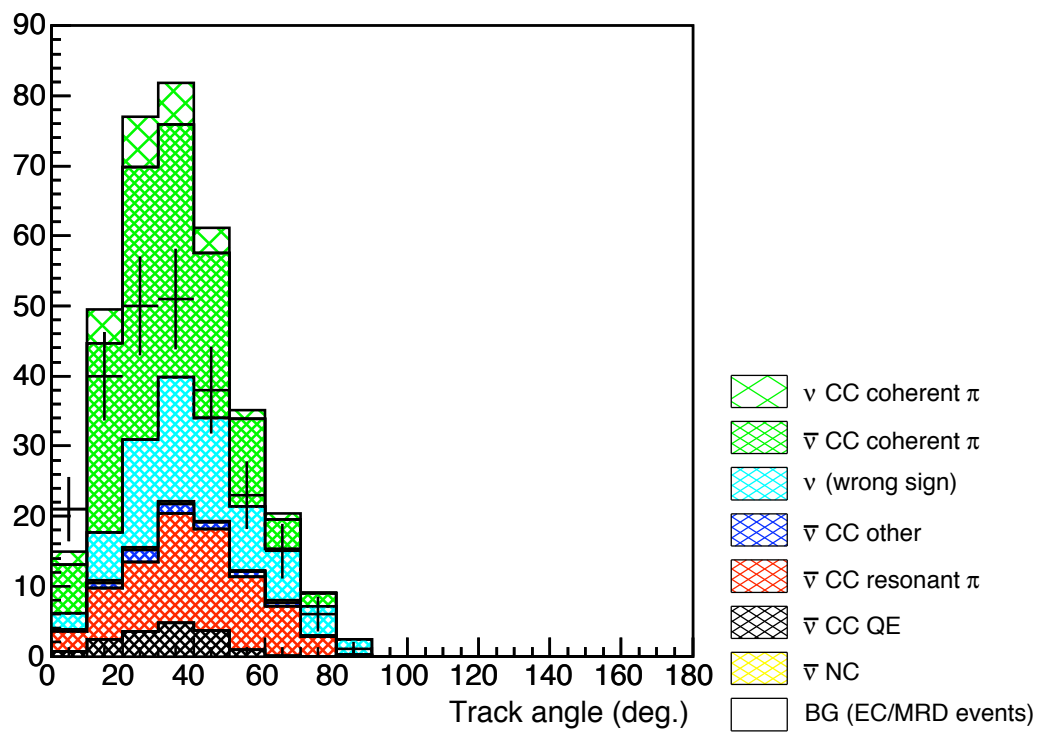

Figure D.6: The pion angular distribution in antineutrino CC coherent pion sample.
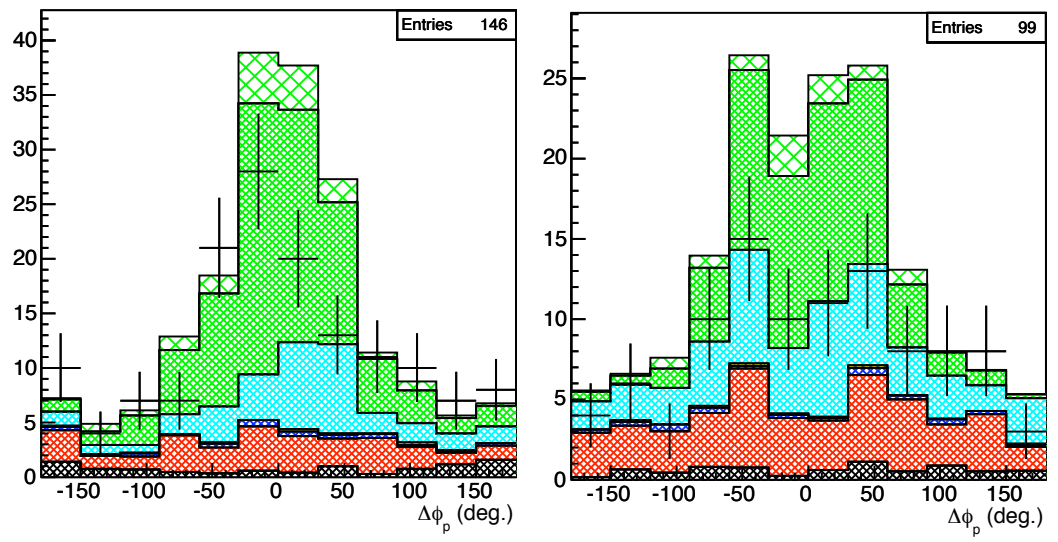

Figure D.7: $\Delta \phi$ distributions for two different regions of the pion scattering angle regions in SciBooNE's antineutrino CC coherent pion sample. The left plot is for $\theta_{\pi}<35^{\circ}$ and the right plot is for $\theta_{\pi} \geq 35^{\circ}$.

The same tests have been performed for antineutrino. Figure D.6 shows the distribution of the pion angle with respect to the beam axis $\left(\theta_{\pi}\right)$ for the antineutrino MRD stopped coherent sample. There are data excess with respect to the background prediction at small pion scattered angle as seen in the neutrino sample. Figure D.7 shows the $\Delta \phi$ distribution for the antineutrino MRD stopped coherent sample. As seen in the neutrino sample, a data excess is found around $\Delta \phi=0$ in the small pion angle sample 
while the data and MC background prediction agree well in the large pion angle sample. The coherent pion signal in the region of the small pion scattering angle is indicated also for the antineutrino data.

\section{D.3 Summary}

The further study of CC coherent pion production is performed both for neutrino and antineutrino data. The antineutrino and neutrino data suggest that pions from $\mathrm{CC}$ coherent pion events tend to be produced more forward than the Rein-Sehgal model prediction. Thus, the kinematic predicted by the Rein-Sehgal model does not match with our observation. In addition, the cross section predicted by the Rein and Sehgal model is smaller than our measurements of the CC coherent pion. From these facts, it is important to measure the cross section and pion kinematic distribution of $\mathrm{NC}$ coherent pion production. 


\section{Bibliography}

[1] Ziro Maki, Masami Nakagawa, and Shoichi Sakata. Remarks on the unified model of elementary particles. Prog. Theor. Phys., Vol. 28, pp. 870-880, 1962.

[2] Y. Ashie, et al. A Measurement of Atmospheric Neutrino Oscillation Parameters by Super-Kamiokande I. Phys. Rev., Vol. D71, p. 112005, 2005.

[3] M. H. Ahn, et al. Measurement of Neutrino Oscillation by the K2K Experiment. Phys. Rev., Vol. D74, p. 072003, 2006.

[4] D. G. Michael, et al. Observation of muon neutrino disappearance with the MINOS detectors and the NuMI neutrino beam. Phys. Rev. Lett., Vol. 97, p. 191801, 2006.

[5] Raymond Davis, Jr., Don S. Harmer, and Kenneth C. Hoffman. Search for neutrinos from the sun. Phys. Rev. Lett., Vol. 20, pp. 1205-1209, 1968.

[6] B. Aharmim, et al. Electron energy spectra, fluxes, and day-night asymmetries of B-8 solar neutrinos from the 391-day salt phase SNO data set. Phys. Rev., Vol. C72, p. 055502, 2005.

[7] J. Hosaka, et al. Solar neutrino measurements in Super-Kamiokande-I. Phys. Rev., Vol. D73, p. 112001, 2006.

[8] S. Abe, et al. Precision Measurement of Neutrino Oscillation Parameters with KamLAND. Phys. Rev. Lett., Vol. 100, p. 221803, 2008.

[9] M. Apollonio, et al. Search for neutrino oscillations on a long base-line at the CHOOZ nuclear power station. Eur. Phys. J., Vol. C27, pp. 331-374, 2003.

[10] F. Boehm, et al. Final results from the Palo Verde Neutrino Oscillation Experiment. Phys. Rev., Vol. D64, p. 112001, 2001.

[11] P. Adamson, et al. Measurement of Neutrino Oscillations with the MINOS Detectors in the NuMI Beam. Phys. Rev. Lett., Vol. 101, p. 131802, 2008.

[12] S. Yamamoto, et al. An improved search for $\mathrm{nu} / \mathrm{mu}-\mathbf{i} \mathrm{nu} / \mathrm{e}$ oscillation in a longbaseline accelerator experiment. Phys. Rev. Lett., Vol. 96, p. 181801, 2006.

[13] P. Adamson, et al. Search for muon-neutrino to electron-neutrino transitions in MINOS. 2009.

[14] Y. Itow, et al. The JHF-Kamioka neutrino project. 2001. 
[15] D. S. Ayres, et al. NOvA proposal to build a 30-kiloton off-axis detector to study neutrino oscillations in the Fermilab NuMI beamline. 2004.

[16] A. A. Aguilar-Arevalo, et al. Measurement of $\nu_{\mu}$ and $\bar{\nu}_{\mu}$ induced neutral current single $\pi^{0}$ production cross sections on mineral oil at $E_{\nu} \sim O(1 G e V) .2009$.

[17] Gian Luigi Fogli and G. Nardulli. A NEW APPROACH TO THE CHARGED CURRENT INDUCED WEAK ONE PION PRODUCTION. Nucl. Phys., Vol. B160, p. 116, 1979.

[18] Dieter Rein and Lalit M. Sehgal. Neutrino Excitation of Baryon Resonances and Single Pion Production. Ann. Phys., Vol. 133, p. 79, 1981.

[19] S. J. Barish, et al. Observation of single pion production by a weak neutral current. Phys. Rev. Lett., Vol. 33, p. 448, 1974.

[20] M. Derrick, et al. STUDY OF SINGLE PION PRODUCTION BY WEAK NEUTRAL CURRENTS IN LOW-ENERGY neutrino d INTERACTIONS. Phys. Rev., Vol. D23, p. 569, 1981.

[21] W. Krenz, et al. Experimental Study of Exclusive One Pion Production in All Neutrino Induced Neutral Current Channels. Nucl. Phys., Vol. B135, p. 45, 1978.

[22] M. Sajjad Athar, Shakeb Ahmad, and S. K. Singh. Charged current anti-neutrino reactions from C-12 at MiniBooNE energies. Phys. Rev., Vol. D75, p. 093003, 2007.

[23] C. Praet, O. Lalakulich, N. Jachowicz, and J. Ryckebusch. Neutrino-induced pion production from nuclei at medium energies. 2008.

[24] M. Martini, M. Ericson, G. Chanfray, and J. Marteau. A unified approach for nucleon knock-out, coherent and incoherent pion production in neutrino interactions with nuclei. 2009.

[25] C. Andreopoulos, et al. The GENIE Neutrino Monte Carlo Generator. 2009.

[26] Artur M. Ankowski and Jan T. Sobczyk. Argon spectral function and neutrino interactions. Phys. Rev., Vol. C74, p. 054316, 2006.

[27] Won-Yong Lee, et al. Single Pion Production in Neutrino and anti-neutrino Reactions. Phys. Rev. Lett., Vol. 38, p. 202, 1977.

[28] S. Nakayama, et al. Measurement of single pi0 production in neutral current neutrino interactions with water by a $1.3-\mathrm{GeV}$ wide band muon neutrino beam. Phys. Lett., Vol. B619, pp. 255-262, 2005.

[29] A. A. Aguilar-Arevalo, et al. First Observation of Coherent $\pi^{0}$ Production in Neutrino Nucleus Interactions with $E_{\nu}<2 \mathrm{GeV}$. Phys. Lett., Vol. B664, pp. 41-46, 2008.

[30] Stephen L. Adler. Tests of the Conserved Vector Current and Partially Conserved Axial-Vector Current Hypotheses in High-Energy Neutrino Reactions. Phys. Rev., Vol. 135, pp. B963-B966, 1964. 
[31] S. S. Gershtein, Yu. Ya. Komachenko, and M. Yu. Khlopov. FAST PION PRODUCTION IN EXCLUSIVE NEUTRINO PROCESSES. Sov. J. Nucl. Phys., Vol. 32, p. 861, 1980.

[32] Dieter Rein and Lalit M. Sehgal. Coherent pi0 Production in Neutrino Reactions. Nucl. Phys., Vol. B223, p. 29, 1983.

[33] A. A. Belkov and B. Z. Kopeliovich. ADLER RELATION AND NEUTRINO PRODUCTION OF SINGLE HADRONS. Sov. J. Nucl. Phys., Vol. 46, p. 499, 1987.

[34] E. A. Paschos, A. Kartavtsev, and G. J. Gounaris. Coherent pion production by neutrinos on nuclei. Phys. Rev., Vol. D74, p. 054007, 2006.

[35] D. Rein and L. M. Sehgal. PCAC and the Deficit of Forward Muons in $p i^{+}$Production by Neutrinos. Phys. Lett., Vol. B657, pp. 207-209, 2007.

[36] S. K. Singh, M. Sajjad Athar, and S. Ahmad. Nuclear effects in neutrino induced coherent pion production at K2K and MiniBooNE. Phys. Rev. Lett., Vol. 96, p. 241801, 2006.

[37] L. Alvarez-Ruso, L. S. Geng, S. Hirenzaki, and M. J. Vicente Vacas. Charged current neutrino induced coherent pion production. Phys. Rev., Vol. C75, p. 055501, 2007.

[38] L. Alvarez-Ruso, L. S. Geng, and M. J. Vicente Vacas. Neutral current coherent pion production. Phys. Rev., Vol. C76, p. 068501, 2007.

[39] H. Faissner, et al. OBSERVATION OF NEUTRINO AND ANTI-NEUTRINO INDUCED COHERENT NEUTRAL PION PRODUCTION OFF Al-27. Phys. Lett., Vol. B125, p. 230, 1983.

[40] E. Isiksal, D. Rein, and J. G. Morfin. EVIDENCE FOR NEUTRINO AND ANTINEUTRINOS INDUCED COHERENT PIO PRODUCTION. Phys. Rev. Lett., Vol. 52, pp. 1096-1099, 1984.

[41] H. J. Grabosch, et al. COHERENT PION PRODUCTION IN NEUTRINO AND ANTI-NEUTRINO INTERACTIONS ON NUCLEI OF HEAVY FREON MOLECULES. Zeit. Phys., Vol. C31, p. 203, 1986.

[42] F. Bergsma, et al. MEASUREMENT OF THE CROSS-SECTION OF COHERENT pi0 PRODUCTION BY MUON NEUTRINO AND ANTI-NEUTRINO NEUTRAL CURRENT INTERACTIONS ON NUCLEI. Phys. Lett., Vol. B157, p. 469, 1985.

[43] P. Vilain, et al. Coherent single charged pion production by neutrinos. Phys. Lett., Vol. B313, pp. 267-275, 1993.

[44] P. Marage, et al. COHERENT SINGLE PION PRODUCTION BY ANTINEUTRINO CHARGED CURRENT INTERACTIONS AND TEST OF PCAC. Z. Phys., Vol. C31, pp. 191-197, 1986.

[45] P. P. Allport, et al. COHERENT PRODUCTION OF pi+ MESONS IN neutrino NEON INTERACTIONS. Z. Phys., Vol. C43, pp. 523-526, 1989. 
[46] M. Aderholz, et al. COHERENT PRODUCTION OF pi+ pi- MESONS BY CHARGED CURRENT INTERACTIONS OF NEUTRINOS AND ANTINEUTRINOS ON NEON NUClEI AT THE TEVATRON. Phys. Rev. Lett., Vol. 63, p. 2349, 1989.

[47] Stephane Willocq, et al. Coherent production of single pions and rho mesons in charged current interactions of neutrinos and anti- neutrinos on neon nuclei at the Fermilab tevatron. Phys. Rev., Vol. D47, pp. 2661-2674, 1993.

[48] M. Hasegawa, et al. Search for coherent charged pion production in neutrino carbon interactions. Phys. Rev. Lett., Vol. 95, p. 252301, 2005.

[49] K. Hiraide, et al. Search for Charged Current Coherent Pion Production on Carbon in a Few-GeV Neutrino Beam. Phys. Rev., Vol. D78, p. 112004, 2008.

[50] A. A. Aguilar-Arevalo, et al. Bringing the SciBar detector to the booster neutrino beam. 2006.

[51] K. Nitta, et al. The K2K SciBar detector. Nucl. Instrum. Meth., Vol. A535, pp. 147-151, 2004.

[52] M. Yoshida, et al. Development of the readout system for the K2K SciBar detector. IEEE Trans. Nucl. Sci., Vol. 51, pp. 3043-3046, 2004.

[53] Y. Arai, et al. Development of a new TDC LSI and a VME module. IEEE Trans. Nucl. Sci., Vol. 49, pp. 1164-1169, 2002.

[54] M. Hasegawa. Ph.d. thesis. 2006.

[55] S. Buontempo, et al. Construction and test of calorimeter modules for the CHORUS experiment. Nucl. Instrum. Meth., Vol. A349, pp. 70-80, 1994.

[56] Katsuki Hiraide. A Study of Charged Current Single Charged Pion Productions on Carbon in a Few-GeV Neutrino Beam. FERMILAB-THESIS-2009-02.

[57] A. A. Aguilar-Arevalo, et al. The Neutrino Flux prediction at MiniBooNE. 2008.

[58] S. Agostinelli, et al. GEANT4: A simulation toolkit. Nucl. Instrum. Meth., Vol. A506, pp. 250-303, 2003.

[59] M. G. Catanesi, et al. Measurement of the production cross-section of positive pions in the collision of $8.9 \mathrm{GeV} / \mathrm{c}$ protons on beryllium. Eur. Phys. J., Vol. C52, pp. 29-53, 2007.

[60] I. Chemakin, et al. Pion Production by Protons on a Thin Beryllium Target at 6.4, 12.3, and 17.5 GeV/c Incident Proton Momenta. Phys. Rev., Vol. C77, p. 015209, 2008 .

[61] J. R. Sanford and C. L. Wang. . BNL Note No. 11299, 1967.

[62] N. V. Mokhov, et al. MARS Code Developments. 1998.

[63] Y. Hayato. NEUT. Nucl. Phys. Proc. Suppl., Vol. 112, pp. 171-176, 2002. 
[64] Gaku Mitsuka. NEUT. AIP Conf. Proc., Vol. 981, pp. 262-264, 2008.

[65] C. H. Llewellyn Smith. Neutrino Reactions at Accelerator Energies. Phys. Rept., Vol. 3, p. 261, 1972.

[66] R. Gran, et al. Measurement of the quasi-elastic axial vector mass in neutrino oxygen interactions. Phys. Rev., Vol. D74, p. 052002, 2006.

[67] A. A. Aguilar-Arevalo, et al. Measurement of muon neutrino quasi-elastic scattering on carbon. Phys. Rev. Lett., Vol. 100, p. 032301, 2008.

[68] L. A. Ahrens, et al. PRECISE DETERMINATION OF SIN**2-theta-w FROM MEASUREMENTS OF THE DIFFERENTIAL CROSS-SECTIONS FOR muonneutrino $\mathrm{p} \rightarrow$ muon-neutrino $\mathrm{p}$ AND anti-muon-neutrino $\mathrm{p} \rightarrow$ anti- muon-neutrino p. Phys. Rev. Lett., Vol. 56, p. 1107, 1986.

[69] Carl H. Albright, C. Quigg, R. E. Shrock, and J. Smith. Neutrino - Proton Elastic Scattering: Implications for Weak Interaction Models. Phys. Rev., Vol. D14, p. 1780, 1976.

[70] R. A. Smith and E. J. Moniz. NEUTRINO REACTIONS ON NUCLEAR TARGETS. Nucl. Phys., Vol. B43, p. 605, 1972.

[71] R. P. Feynman, M. Kislinger, and F. Ravndal. Current matrix elements from a relativistic quark model. Phys. Rev., Vol. D3, pp. 2706-2732, 1971.

[72] Ch. Berger and L. M. Sehgal. Lepton Mass Effects in Single Pion Production by Neutrinos. Phys. Rev., Vol. D76, p. 113004, 2007.

[73] Konstantin S. Kuzmin, Vladimir V. Lyubushkin, and Vadim A. Naumov. Lepton polarization in neutrino nucleon interactions. Mod. Phys. Lett., Vol. A19, pp. 28152829, 2004.

[74] D. Rein. ANGULAR DISTRIBUTION IN NEUTRINO INDUCED SINGLE PION PRODUCTION PROCESSES. Z. Phys., Vol. C35, pp. 43-64, 1987.

[75] T. Kitagaki, et al. CHARGED CURRENT EXCLUSIVE PION PRODUCTION IN NEUTRINO DEUTERIUM INTERACTIONS. Phys. Rev., Vol. D34, pp. 2554$2565,1986$.

[76] S. K. Singh, M. J. Vicente-Vacas, and E. Oset. Nuclear effects in neutrinoproduction of Delta at intermediate energies. Phys. Lett., Vol. B416, pp. 23-28, 1998.

[77] M. Gluck, E. Reya, and A. Vogt. Dynamical parton distributions revisited. Eur. Phys. J., Vol. C5, pp. 461-470, 1998.

[78] A. Bodek and U. K. Yang. Modeling neutrino and electron scattering cross sections in the few GeV region with effective LO PDFs. 2003.

[79] Masayuki Nakahata, et al. ATMOSPHERIC NEUTRINO BACKGROUND AND PION NUCLEAR EFFECT FOR KAMIOKA NUCLEON DECAY EXPERIMENT. J. Phys. Soc. Jap., Vol. 55, p. 3786, 1986. 
[80] Torbjorn Sjostrand. High-energy physics event generation with PYTHIA 5.7 and JETSET 7.4. Comput. Phys. Commun., Vol. 82, pp. 74-90, 1994.

[81] P. Musset and J. P. Vialle. Neutrino Physics with GARGAMELLE. Phys. Rept., Vol. 39, pp. 1-130, 1978.

[82] Jihn E. Kim, Paul Langacker, M. Levine, and H. H. Williams. A Theoretical and Experimental Review of the Weak Neutral Current: A Determination of Its Structure and Limits on Deviations from the Minimal SU(2)-L x U(1) Electroweak Theory. Rev. Mod. Phys., Vol. 53, p. 211, 1981.

[83] C. W. De Jager, H. De Vries, and C. De Vries. Nuclear charge and magnetization density distribution parameters from elastic electron scattering. Atom. Data Nucl. Data Tabl., Vol. 14, pp. 479-508, 1974.

[84] L. L. Salcedo, E. Oset, M. J. Vicente-Vacas, and C. Garcia-Recio. COMPUTER SIMULATION OF INCLUSIVE PION NUCLEAR REACTIONS. Nucl. Phys., Vol. A484, p. 557, 1988.

[85] D. Ashery, et al. TRUE ABSORPTION AND SCATTERING OF PIONS ON NUCLEI. Phys. Rev., Vol. C23, pp. 2173-2185, 1981.

[86] Glenn Rowe, Martin Salomon, and Rubin H. Landau. AN ENERGY DEPENDENT PHASE SHIFT ANALYSIS OF PION - NUCLEON SCATTERING BELOW 400MeV. Phys. Rev., Vol. C18, pp. 584-589, 1978.

[87] H. W. Bertini. Nonelastic interactions of nucleons and pi mesons with complex nuclei at energies below 3 gev. Phys. Rev., Vol. C6, pp. 631-659, 1972.

[88] S. J. Lindenbaum and R. M. Sternheimer. Isobaric nucleon model for pion production in nucleon- nucleon collisions. Phys. Rev., Vol. 105, pp. 1874-1879, 1957.

[89] Aatos Heikkinen, Nikita Stepanov, and Johannes Peter Wellisch. Bertini intranuclear cascade implementation in Geant4. 2003.

[90] Colin Wilkin, et al. A comparison of pi+ and pi- total cross-sections of light nuclei near the 3-3 resonance. Nucl. Phys., Vol. B62, pp. 61-85, 1973.

[91] A. S. Clough, et al. Pion-Nucleus Total Cross-Sections from 88-MeV to 860- MeV. Nucl. Phys., Vol. B76, p. 15, 1974.

[92] A. S. Carroll, et al. Pion-Nucleus Total Cross-Sections in the (3,3) Resonance Region. Phys. Rev., Vol. C14, pp. 635-638, 1976.

[93] R. D. Ransome, et al. Pion absorption in heavy nuclei. Phys. Rev., Vol. C45, pp. 509-513, 1992.

[94] M. K. Jones, et al. Pion absorption above the Delta (1232) resonance. Phys. Rev., Vol. C48, pp. 2800-2817, 1993.

[95] H. Maesaka. Ph.d. thesis. 2005. 
[96] A. Rodriguez, et al. Measurement of single charged pion production in the chargedcurrent interactions of neutrinos in a $1.3 \mathrm{GeV}$ wide band beam. Phys. Rev., Vol. D78, p. 032003, 2008.

[97] Veronique Bernard, Latifa Elouadrhiri, and Ulf. G. Meissner. Axial structure of the nucleon. J. Phys., Vol. G28, pp. R1-R35, 2002.

[98] G. D'Agostini. A Multidimensional unfolding method based on Bayes' theorem. Nucl. Instrum. Meth., Vol. A362, pp. 487-498, 1995.

[99] K. S. Lackner. COHERENT MESON PRODUCTION AS A TEST FOR NEUTRAL WEAK CURRENTS OF EXOTIC SPACE-TIME STRUCTURE. Nucl. Phys., Vol. B153, p. 526, 1979.

[100] D. Drakoulakos, et al. Proposal to perform a high-statistics neutrino scattering experiment using a fine-grained detector in the NuMI beam. 2004.

[101] K. Hiraide. Search for neutrino charged current coherent pion production at SciBooNE. 2009.

[102] Hide-Kazu Tanaka. Search for Antineutrino Charged Current Coherent Pion Production at SciBooNE. 2009. 